SUPPORTING INFORMATION

\title{
AoiQ Catalyzes Geminal Dichlorination of 1,3-Diketone Natural
}

\section{Products}

Mengting Liu, ${ }^{1,3}$ Masao Ohashi, ${ }^{*}$ Yiu-Sun Hung, ${ }^{1}$ Kirstin Scherlach, ${ }^{4}$ Kenji Watanabe, ${ }^{6}$ Christian Hertweck, ${ }^{4,5^{*}}$ Yi Tang ${ }^{1,2^{*}}$

${ }^{1}$ Department of Chemical and Biomolecular Engineering and ${ }^{2}$ Department of Chemistry and Biochemistry University of California, Los Angeles, California 90095, USA. ${ }^{3}$ Hubei Key Laboratory of Natural Medicinal Chemistry and Resource Evaluation, School of Pharmacy, Tongji Medical College, Huazhong University of Science and Technology, Wuhan 430030. ${ }^{4}$ Leibniz Institute for Natural Product Research and Infection Biology - HKI, 07745 Jena, Germany. ${ }^{5}$ Faculty of Biological Sciences, Friedrich Schiller University Jena, 07743 Jena, Germany. ${ }^{6}$ Department of Pharmaceutical Sciences, University of Shizuoka, Shizuoka 422-8526, Japan. 


\section{Table of Contents}

$\begin{array}{lr}\text { Experimental procedures } & \text { S6 }\end{array}$

1. Strains and general culture conditions. $\quad$ S6

2. General DNA manipulation techniques. $\quad$ S6

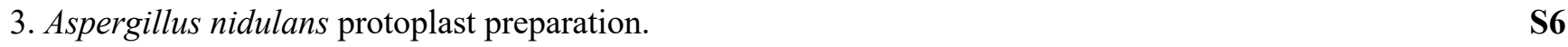

$\begin{array}{ll}\text { 4. Heterologous expression of the dia and aoi gene clusters in A. nidulans. } & \text { S7 }\end{array}$

$\begin{array}{lr}\text { 5. Compound identification, isolation, and characterization. } & \text { S7 }\end{array}$

6. Spectroscopic analyses.

7. Reconstitution of chlorinated orthosporins (1-3) pathway in S. cerevisiae.

8. Expression and purification of AoiQ (FDH-MT) and dissected AoiQ (FDH) from S. cerevisiae. $\quad$ S11

9. Expression and purification of AoiQ (MT), DiaC, NgvF and Fre from E. coli BL21(DE3).

10. Biotransformation assays in S. cerevisiae.

11. In vitro assay for DiaC. $\quad$ S12

12. In vitro assay for AoiQ (MT) or NgvF. $\quad$ S12

13. In vitro assays for AoiQ (FDH).

14. Coupled in vitro activity assay of AoiQ (FDH), DiaC, and/or AoiQ (MT).

15. Chemical semi-synthesis of compound $\mathbf{1 5 .} \quad \mathbf{S 1 3}$

16. Sequence information. $\quad$ S13

Supplementary Tables

Table S1. Bioinformatic analysis of dia gene cluster. $\quad$ S15

$\begin{array}{lr}\text { Table S2. Primers used in this study. } & \text { S16 }\end{array}$

Table S3. Plasmids used in this study.

Table S4. Spectroscopic data of compound 1.

$\begin{array}{lr}\text { Table S5. Spectroscopic data of compound 2. } & \text { S21 }\end{array}$

Table S6. Spectroscopic data of compound 3. $\quad$ S22

$\begin{array}{ll}\text { Table S7. Spectroscopic data of compound 4. } & \text { S23 }\end{array}$

$\begin{array}{lr}\text { Table S8. Spectroscopic data of compound 5. } & \text { S24 }\end{array}$

$\begin{array}{ll}\text { Table S9. Spectroscopic data of compound 6. } & \text { S25 }\end{array}$

$\begin{array}{lr}\text { Table S10. Spectroscopic data of compound 7. } & \text { S26 }\end{array}$

$\begin{array}{lr}\text { Table S11. Spectroscopic data of compound } 8 . & \text { S27 }\end{array}$

$\begin{array}{lr}\text { Table S12. Spectroscopic data of compound } 9 . & \text { S28 }\end{array}$

Table S13. Spectroscopic data of compound 10.

Table S14. Spectroscopic data of compound 12.

$\begin{array}{ll}\text { Table S15. Spectroscopic data of compound 13. } & \text { S31 }\end{array}$

Table S16. Spectroscopic data of compound 14.

Table S17. Spectroscopic data of compound 15.

$\begin{array}{ll}\text { Table S18. Spectroscopic data of compound } 16 . & \text { S34 }\end{array}$ 
Table S19. Spectroscopic data of compound 19.

Table S20. Spectroscopic data of compound 20.

Table S21. Spectroscopic data of compound 21.

Table S22. Spectroscopic data of compound 22.

S38

Table S23. Spectroscopic data of compound 23.

\section{Supplementary Figures}

Figure S1. Natural products with halogenation of the aromatic C-H using FDH.

Figure S2. Homologous biosynthetic gene clusters of dia and $n g v$ from NCBI database.

Figure S3. Functional analysis of AoiQ homologs from different fungi in S. cerevisiae BJ5464. $\quad$ S42

Figure S4. Feeding 6 to $S$. cerevisiae expressing AoiQ with or without DiaC. $\quad \mathbf{S 4 3}$

Figure S5. Heterologous expression of aoi and dia genes in A. nidulans. $\quad$ S44

Figure S6. The metabolites from A. nidulans transformants expressing dia and homologous clusters. $\quad \mathbf{S 4 5}$

Figure S7. SDS-PAGE gels of purified proteins used in this study.

Figure S8. LC/MS analysis of the supernatant of boiled AoiQ showed FAD is copurified with AoiQ. $\quad$ S47

$\begin{array}{ll}\text { Figure S9. In vitro DiaC cofactor requirements with compound } 6 \text { as substrate. } & \mathbf{S 4 8}\end{array}$

Figure S10. LC/MS analysis of extracts from $A$. nidulans expressing diaAB and dissected $a o i Q(\mathrm{FDH}) . \quad$ S49

Figure S11. Feeding 6, 8, and 10 to $S$. cerevisiae expressing aoi $Q$ with diaC genes.

Figure S12. LC/MS analysis of enzymatic reaction of AoiQ with compound $6 . \quad 551$

Figure S13. The multiple sequence alignments of AoiQ with reported fungal origin FDHs.

Figure S14. HPLC-MS profiles of compound 1, 2, 3, 5, 15, 16, 19, 20, 21, 22 and $23 . \quad S 53$

Figure S15. LC/MS analysis of enzymatic reaction of AoiQ (FDH) with compound 6.

Figure S16. Feeding compound $\mathbf{6}$ to $S$. cerevisiae expressing dissected aoiQ $(F D H)$.

Figure S17. In vitro verification of MT domain involvement, and lysine residue requirement for AoiQ. $\quad$ S56

Figure S18. The flavin reductase activity of AoiQ-FDH for NADH and NADPH.

Figure S19. HPLC analysis of enzymatic reaction of AoiQ (MT) with compound 2.

Figure S20. LC/MS analysis of enzymatic reaction of AoiQ (FDH) with synthetic 15. S59

Figure S21. LC/MS analysis of enzymatic reaction of DiaC with $\mathbf{6}$ or $\mathbf{1 6}$ as substrate. $\quad$ S60

Figure S22. Kinetic parameters of DiaC-catalyzed the conversion of $\mathbf{1 6}$ to $\mathbf{5}$ and $\mathbf{6}$ to $\mathbf{1 0}$ S61

Figure S23. UV profiles of compound of 1, 2, 3, 12, 19, 20, 21, 22, and 23.

Figure S24. LC/MS analysis of enzymatic reaction of AoiQ with 2, 4 and $\mathbf{1 2} . \quad$ S63

Figure S25. LC/MS analysis of A. nidulans expressing aoiG and aoiQ $(F D H)$ S64

Figure S26. LC/MS analysis of enzymatic reaction of AoiQ (FDH) domain with 8 and $9 . \quad$ S65

Figure S27. LC/MS analysis of enzymatic reaction of AoiQ (FDH) using bromo as halogen donor. $\quad$ S66

Figure S28. ${ }^{1} \mathrm{H}$ NMR spectrum of compound $\mathbf{1}$ in DMSO- $d_{6} . \quad$ S67

$\begin{array}{lr}\text { Figure S29. }{ }^{13} \mathrm{C} \text { NMR spectrum of compound } \mathbf{1} \text { in DMSO- } d_{6} . & \text { S68 }\end{array}$

Figure S30. ${ }^{1} \mathrm{H}$ NMR spectrum of compound $\mathbf{2}$ in $\mathrm{CD}_{3} \mathrm{OD}$.

Figure S31. ${ }^{13} \mathrm{C}$ NMR spectrum of compound $\mathbf{2}$ in $\mathrm{CD}_{3} \mathrm{OD}$. $\quad$ S70

$\begin{array}{ll}\text { Figure S32. HSQC spectrum of compound } \mathbf{2} \text { in } \mathrm{CD}_{3} \mathrm{OD} \text {. } & \mathbf{S 7 1}\end{array}$ 
Figure S33. HMBC spectrum of compound 2 in $\mathrm{CD}_{3} \mathrm{OD}$.

Figure S34. COSY spectrum of compound 2 in $\mathrm{CD}_{3} \mathrm{OD}$.

Figure S35. ${ }^{1} \mathrm{H}$ NMR spectrum of compound 3 in $\left(\mathrm{CD}_{3}\right)_{2} \mathrm{CO}$.

Figure S36. ${ }^{13} \mathrm{C}$ NMR spectrum of compound 3 in $\left(\mathrm{CD}_{3}\right)_{2} \mathrm{CO}$.

S75

Figure S37. ${ }^{1} \mathrm{H}$ NMR spectrum of compound 4 in $\left(\mathrm{CD}_{3}\right)_{2} \mathrm{CO}$.

S76

Figure S38. ${ }^{13} \mathrm{C}$ NMR spectrum of compound 4 in $\left(\mathrm{CD}_{3}\right)_{2} \mathrm{CO}$.

S77

Figure S39. ${ }^{1} \mathrm{H}$ NMR spectrum of compound 5 in $\mathrm{CDCl}_{3}$.

S78

Figure S40. ${ }^{13} \mathrm{C}$ NMR spectrum of compound 5 in $\mathrm{CDCl}_{3}$.

S79

Figure S41. ${ }^{1} \mathrm{H}$ NMR spectrum of compound 6 in DMSO- $d_{6}$.

S80

Figure S42. ${ }^{13} \mathrm{C}$ NMR spectrum of compound 6 in DMSO- $d_{6}$.

Figure S43. HSQC spectrum of compound 6 in DMSO- $d_{6}$.

Figure S44. HMBC spectrum of compound 6 in DMSO- $d_{6}$.

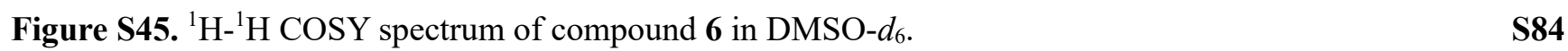

Figure S46. ${ }^{1} \mathrm{H}$ NMR spectrum of compound 7 in DMSO- $d_{6}$.

Figure S47. The ${ }^{13} \mathrm{C}$ NMR spectrum of compound 7 in DMSO- $d_{6} . \quad 386$

$\begin{array}{ll}\text { Figure S48. The }{ }^{1} \mathrm{H} \text { NMR spectrum of compound } \mathbf{8} \text { in } \mathrm{CD}_{3} \mathrm{OD} \text {. } & \mathbf{S 8 7}\end{array}$

Figure S49. The ${ }^{13} \mathrm{C}$ NMR spectrum of compound $\mathbf{8}$ in $\mathrm{CD}_{3} \mathrm{OD}$.

Figure S50. HSQC spectrum of compound $\mathbf{8}$ in $\mathrm{CD}_{3} \mathrm{OD}$.

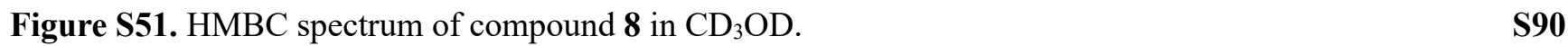

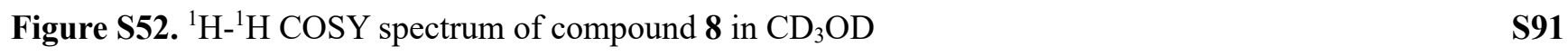

Figure S53. ${ }^{1} \mathrm{H}$ NMR spectrum of compound $\mathbf{9}$ in $\mathrm{CD}_{3} \mathrm{OD}$.

Figure S54. ${ }^{13} \mathrm{C}$ NMR spectrum of compound $\mathbf{9}$ in $\mathrm{CD}_{3} \mathrm{OD} . \quad \mathbf{S 9 3}$

Figure S55. HSQC spectrum of compound 9 in $\mathrm{CD}_{3} \mathrm{OD}$.

Figure S56. HMBC spectrum of compound $\mathbf{9}$ in $\mathrm{CD}_{3} \mathrm{OD}$.

Figure S57. ${ }^{1} \mathrm{H}-{ }^{1} \mathrm{H}$ COSY spectrum of compound 9 in $\mathrm{CD}_{3} \mathrm{OD} . \quad \mathbf{S 9 6}$

Figure S58. ${ }^{1} \mathrm{H}$ NMR spectrum of compound $\mathbf{1 0}$ in $\mathrm{CD}_{3} \mathrm{OD}$.

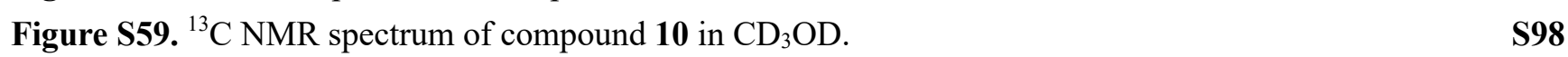

Figure S60. HSQC spectrum of compound $\mathbf{1 0}$ in $\mathrm{CD}_{3} \mathrm{OD}$.

Figure S61. HMBC spectrum of compound 10 in $\mathrm{CD}_{3} \mathrm{OD} . \quad \mathbf{S 1 0 0}$

Figure S62. ${ }^{1} \mathrm{H}-{ }^{1} \mathrm{H}$ COSY spectrum of compound $\mathbf{1 0}$ in $\mathrm{CD}_{3} \mathrm{OD}$.

Figure S63. The ${ }^{1} \mathrm{H}$ NMR spectrum of compound 12 in $\mathrm{CD}_{3} \mathrm{OD}$.

Figure S64. The ${ }^{13} \mathrm{C}$ NMR spectrum of compound 12 in $\mathrm{CD}_{3} \mathrm{OD}$.

$\begin{array}{ll}\text { Figure S65. HSQC spectrum of compound } 12 \text { in } \mathrm{CD}_{3} \mathrm{OD} & \mathbf{S 1 0 4}\end{array}$

Figure S66. HMBC spectrum of compound 12 in $\mathrm{CD}_{3} \mathrm{OD}$.

Figure S67. ${ }^{1} \mathrm{H}-{ }^{1} \mathrm{H}$ COSY spectrum of compound 12 in $\mathrm{CD}_{3} \mathrm{OD}$.

Figure S68. ${ }^{1} \mathrm{H}$ NMR spectrum of compound 13 in $\mathrm{CDCl}_{3}$.

Figure S69. ${ }^{13} \mathrm{C}$ NMR spectrum of compound 13 in $\mathrm{CDCl}_{3}$.

Figure S70. HSQC spectrum of compound 13 in $\mathrm{CDCl}_{3}$.

$\begin{array}{lr}\text { Figure S71. HMBC spectrum of compound } \mathbf{1 3} \text { in } \mathrm{CDCl}_{3} \text {. } & \mathbf{S 1 1 0}\end{array}$ 
Figure S72. ${ }^{1} \mathrm{H}-{ }^{1} \mathrm{H}$ COSY spectrum of compound 13 in $\mathrm{CDCl}_{3}$.

Figure S73. ${ }^{1} \mathrm{H}$ NMR spectrum of compound 14 in $\mathrm{CDCl}_{3}$.

Figure S74. ${ }^{13} \mathrm{C}$ NMR spectrum of compound 14 in $\mathrm{CDCl}_{3}$.

Figure S75. ${ }^{1} \mathrm{H}$ NMR spectrum of compound 15 in acetonitrile- $d_{3}$.

S114

Figure S76. The ${ }^{13} \mathrm{C}$ NMR spectrum of compound 15 in acetonitrile- $d_{3}$.

S115

Figure S77. HSQC spectrum of compound 15 in acetonitrile- $d_{3}$.

S116

Figure S78. HMBC spectrum of compound 15 in acetonitrile- $d_{3}$.

$\mathbf{S 1 1 7}$

Figure S79. ${ }^{1} \mathrm{H}-{ }^{1} \mathrm{H}$ COSY spectrum of compound 15 in acetonitrile- $d_{3}$.

Figure S80. ${ }^{1} \mathrm{H}$ NMR spectrum of compound $\mathbf{1 6}$ in acetonitrile- $d_{3}$.

Figure S81. ${ }^{13} \mathrm{C}$ NMR spectrum of compound 16 in acetonitrile- $d_{3}$.

Figure S82. HSQC spectrum of compound $\mathbf{1 6}$ in acetonitrile- $d_{3}$.

Figure S83. HMBC spectrum of compound 16 in acetonitrile- $d_{3}$.

S122

Figure S84. ${ }^{1} \mathrm{H}-{ }^{1} \mathrm{H}$ COSY spectrum of compound $\mathbf{1 6}$ in acetonitrile- $d_{3}$.

S123

Figure S85. The ${ }^{1} \mathrm{H}$ NMR spectrum of compound 19 in $\mathrm{CD}_{3} \mathrm{OD}$.

S124

Figure S86. The ${ }^{13} \mathrm{C}$ NMR spectrum of compound 19 in $\mathrm{CD}_{3} \mathrm{OD}$.

S125

Figure S87. HSQC spectrum of compound 19 in $\mathrm{CD}_{3} \mathrm{OD}$.

S126

Figure S88. $\mathrm{HMBC}$ spectrum of compound 19 in $\mathrm{CD}_{3} \mathrm{OD}$.

Figure S89. ${ }^{1} \mathrm{H}-{ }^{1} \mathrm{H}$ COSY spectrum of compound 19 in $\mathrm{CD}_{3} \mathrm{OD}$

Figure $\mathbf{S 9 0}{ }^{1} \mathrm{H}$ NMR spectrum of compound 20 in $\mathrm{CD}_{3} \mathrm{OD}$.

S129

Figure S91. ${ }^{13} \mathrm{C}$ NMR spectrum of compound 20 in $\mathrm{CD}_{3} \mathrm{OD}$.

S130

Figure S92. $\mathrm{HSQC}$ spectrum of compound 20 in $\mathrm{CD}_{3} \mathrm{OD}$.

S131

Figure S93. HMBC spectrum of compound 20 in $\mathrm{CD}_{3} \mathrm{OD}$.

S132

Figure S94. ${ }^{1} \mathrm{H}-{ }^{1} \mathrm{H}$ COSY spectrum of compound 20 in $\mathrm{CD}_{3} \mathrm{OD}$.

Figure S95. ${ }^{1} \mathrm{H}$ NMR spectrum of compound 21 in $\mathrm{CD}_{3} \mathrm{OD}$.

S134

Figure S96. ${ }^{13} \mathrm{C}$ NMR spectrum of compound 21 in $\mathrm{CD}_{3} \mathrm{OD}$.

S135

Figure S97. $\mathrm{HSQC}$ spectrum of compound 21 in $\mathrm{CD}_{3} \mathrm{OD}$.

S136

Figure S98. $\mathrm{HMBC}$ spectrum of compound 21 in $\mathrm{CD}_{3} \mathrm{OD}$.

S137

Figure S99. ${ }^{1} \mathrm{H}-{ }^{1} \mathrm{H}$ COSY spectrum of compound 21 in $\mathrm{CD}_{3} \mathrm{OD}$.

S138

Figure S100. ${ }^{1} \mathrm{H}$ NMR spectrum of compound 22 in $\mathrm{CD}_{3} \mathrm{OD}$.

S139

Figure S101. ${ }^{13} \mathrm{C}$ NMR spectrum of compound 22in $\mathrm{CD}_{3} \mathrm{OD}$.

S140

Figure S102. HSQC spectrum of compound 22 in $\mathrm{CD}_{3} \mathrm{OD}$.

S141

Figure S103. HMBC spectrum of compound 22 in $\mathrm{CD}_{3} \mathrm{OD}$.

S142

Figure S104. ${ }^{1} \mathrm{H}-{ }^{1} \mathrm{H}$ COSY spectrum of compound 22 in $\mathrm{CD}_{3} \mathrm{OD}$.

S143

Figure S105. ${ }^{1} \mathrm{H}$ NMR spectrum of compound 23 in $\mathrm{CD}_{3} \mathrm{OD}$.

S144

Figure S106. ${ }^{13} \mathrm{C}$ NMR spectrum of compound 23 in $\mathrm{CD}_{3} \mathrm{OD}$.

S145

Figure S107. HSQC spectrum of compound 23 in $\mathrm{CD}_{3} \mathrm{OD}$.

Figure S108. HMBC spectrum of compound 23 in $\mathrm{CD}_{3} \mathrm{OD}$.

S147

Figure S109. ${ }^{1} \mathrm{H}-{ }^{-1} \mathrm{H}$ COSY spectrum of compound 23 in $\mathrm{CD}_{3} \mathrm{OD}$. $\quad \mathbf{S 1 4 8}$

Figure S110. NOESY spectrum of compound 23 in $\mathrm{CD}_{3} \mathrm{OD}$. 


\section{Experimental procedures}

\section{Strains and culture conditions}

Penicillium nalgiovense NRRL911 was obtained from the Agricultural Research Service Culture Collection (NRRL). Aspergillus oryzae RIB40 (NBRC100959) was acquired from the NBRC culture collections Japan. Trichoderma virens Gv29-8 was acquired from the Fungal Genetics Stock Center (FGSC). Aspergillus nidulans A1145 $\Delta \mathrm{EM} \Delta \mathrm{ST}$ host was previously developed in our lab. All fungal strains except for $A$. nidulans were maintained on potato dextrose agar (PDA, BD) at $28^{\circ} \mathrm{C}$ for cell proliferation or in liquid PDB medium (PDA medium without agar) for isolation of genomic DNA. A. nidulans A1145 ${ }^{1}$ was grown at $28^{\circ} \mathrm{C}$ in CD media $(1 \mathrm{~L}: 10 \mathrm{~g}$ Glucose, 50 $\mathrm{mL} 20 \times$ Nitrate salts, $1 \mathrm{~mL}$ Trace elements, $\mathrm{pH} 6.5$, and $20 \mathrm{~g} / \mathrm{L}$ Agar for solid cultivation) for sporulation or in CDST media (1L: $20 \mathrm{~g}$ Starch, $20 \mathrm{~g}$ Casamino acids, $50 \mathrm{~mL}$ 20x Nitrate salts, $1 \mathrm{~mL}$ Trace elements, $\mathrm{pH} 6.5)^{2}$ for heterologous expression of gene cluster, compounds production and RNA extraction. All E. coli strain were cultured in LB media at $37^{\circ} \mathrm{C}$, and yeast strains were cultured in YPD (yeast extract $1 \%$, peptone $2 \%$, glucose $2 \%$ ) media at $28^{\circ} \mathrm{C}$.

\section{General DNA manipulation techniques}

All the DNA manipulations in this study were conducted according to the manufacturer's protocol. E. coli TOP10 and E. coli XL-1 were used for cloning, following standard recombinant DNA techniques. DNA restriction enzymes were used as recommended by the manufacturer (New England Biolabs, NEB). Genomic DNA from all fungal strains was prepared using LETS isolation buffer (10 mM Tris-HCI, pH 8.0, $20 \mathrm{mM}$ EDTA, 0.5\% SDS, 0.1 $\mathrm{M} \mathrm{LiCl)}$. PCR was performed using Phusion High-Fidelity DNA Polymerase (NEB). The gene-specific primers are listed in Table S2. PCR products were confirmed by DNA sequencing. E. coli BL21(DE3) (Novagen) was used for protein expression. Saccharomyces cerevisiae strain BJ5464-NpgA (MATa ura3-52 his3-4200 leu2-41 trp1 pep4::HIS3 prb1 $\triangle 1.6 R$ canl GAL) was used as the yeast host for in vivo homologous recombination to construct the $A$. nidulans plasmids. ${ }^{3}$ For isolation of RNA from $A$. nidulans transformants, the strains were grown on CD liquid for 4 days at $28{ }^{\circ} \mathrm{C}$. The RNA extraction steps were performed using RiboPure ${ }^{\mathrm{TM}}$ Yeast RNA Isolation Kit (Ambion) following the manufacturer's instructions. Residual genomic DNA in the extracts was digested by DNase I (2 U/ $\mu \mathrm{L})$ (Invitrogen) at $37^{\circ} \mathrm{C}$ for 4 hours. SuperScript III FirstStrand Synthesis System (Invitrogen) was used for cDNA synthesis with Oligo-dT primers following directions from the user manual.

\section{Preparation of protoplast of $A$. nidulans}

The general fungal transformation method has been previously described in detail elsewhere. Briefly, for the transformation of Aspergillus nidulans A1145 $\Delta \mathrm{EM} \Delta \mathrm{ST}$, spores were inoculated into $50 \mathrm{~mL}$ liquid $\mathrm{CD}$ media in a $125 \mathrm{~mL}$ flask and germinated at $30{ }^{\circ} \mathrm{C}$ shaking at $250 \mathrm{rpm}$ for $\sim 9 \mathrm{~h}$. The $20 \times$ Nitrate salts solution was prepared by dissolving $120 \mathrm{~g} \mathrm{NaNO}_{3}, 10.4 \mathrm{~g} \mathrm{KCl}, 10.4 \mathrm{~g} \mathrm{MgSO}_{4} \cdot 7 \mathrm{H}_{2} \mathrm{O}$, and $30.4 \mathrm{~g} \mathrm{KH}_{2} \mathrm{PO}_{4}$ in $1 \mathrm{~L}$ double distilled water. The trace elements solution (100 mL) contained $2.20 \mathrm{~g} \mathrm{ZnSO}_{4} \bullet 7 \mathrm{H}_{2} \mathrm{O}, 1.10 \mathrm{~g} \mathrm{H}_{3} \mathrm{BO}_{3}, 0.50 \mathrm{~g} \mathrm{MnCl}_{2} \bullet 4 \mathrm{H}_{2} \mathrm{O}, 0.16 \mathrm{~g}$ $\mathrm{FeSO}_{4} \cdot 7 \mathrm{H}_{2} \mathrm{O}, 0.16 \mathrm{~g} \mathrm{CoCl}_{2} \cdot 5 \mathrm{H}_{2} \mathrm{O}, 0.16 \mathrm{~g} \mathrm{CuSO}_{4} \cdot 5 \mathrm{H}_{2} \mathrm{O}$, and $0.11 \mathrm{~g}\left(\mathrm{NH}_{4}\right)_{6} \mathrm{Mo}_{7} \mathrm{O}_{24} \bullet 4 \mathrm{H}_{2} \mathrm{O}$. The dropout components for selection for the three expression vectors were uracil/uridine, pyridoxine and riboflavin. A. nidulans A1145 $\Delta \mathrm{EM}$ 
was initially grown on $\mathrm{CD}$ agar plates containing $10 \mathrm{mM}$ uridine, $5 \mathrm{mM}$ uracil, $0.5 \mu \mathrm{g} / \mathrm{mL}$ pyridoxine $\mathrm{HCl}$ and 2.5 $\mu \mathrm{g} / \mathrm{mL}$ riboflavin at $37^{\circ} \mathrm{C}$ for 5 days. The germinated spores were harvested by centrifugation at 3,500 $\mathrm{g}$ for $10 \mathrm{~min}$, and washed with Osmotic buffer $\left(10 \mathrm{~mL}, 1.2 \mathrm{M} \mathrm{MgSO}_{4}, 10 \mathrm{mM}\right.$ sodium phosphate buffer, $\mathrm{pH}$ 5.8). The mycelia were then mixed with Osmotic buffer (10 mL, $30 \mathrm{mg}$ lysing enzymes from Trichoderma, $20 \mathrm{mg}$ Yatalase) in a 125 $\mathrm{mL}$ flask. Protoplasts were prepared by incubating the mixture overnight at $30{ }^{\circ} \mathrm{C}$ with gentle shaking at $80 \mathrm{rpm}$. Cells were collected in a $30 \mathrm{~mL}$ Corex tube and overlaid gently by $10 \mathrm{~mL}$ of Trapping buffer $(0.6 \mathrm{M}$ sorbitol, $0.1 \mathrm{M}$ Tris HCI, $\mathrm{pH}$ 7.0). Centrifugation at 3,500 $\mathrm{g}$ for $15 \mathrm{~min}$ at $4{ }^{\circ} \mathrm{C}$ layered the protoplasts at the interface of the two buffers. The protoplasts were then pipetted to a sterile $15 \mathrm{~mL}$ falcon tube and washed with STC buffer $(10 \mathrm{~mL}, 1.2$ $\mathrm{M}$ sorbitol, $10 \mathrm{mM} \mathrm{CaCl}_{2}, 10 \mathrm{mM}$ Tris-HCI $\mathrm{pH}$ 7.5). The protoplasts were resuspended in STC buffer (1 mL).

\section{Heterologous expression of $\mathbf{d i a}$ and aoi gene clusters in $\boldsymbol{A}$. nidulans}

All $A$. nidulans plasmids for heterologous expression in $A$. nidulans A1145 $\Delta \mathrm{EM} \Delta \mathrm{ST}$ were built using the plasmids pYTU, pYTP, and pYTR ${ }^{4}$ with auxotrophic markers for uracil ( $p y r G$ ), pyridoxine (pyroA), and riboflavin (riboB), respectively, as backbones to insert genes. gpdA promoters from A. nidulans, and Penicillium oxalicum (POgpdA), Penicillium expansum (PEgpdA) were amplified by PCR.

To construct the pML2001 plasmid, full length aoiG was amplified by PCR with two sets of primers of pML2001 F1/R1, pML2001 F2/R2 (Table S1). The two overlapping DNA fragments and PacI/SwaI-digested pYTU expression vector were transformed into yeast for homologous recombination. Plasmids were extracted from yeast using Zymoprep ${ }^{\mathrm{TM}}$ Yeast Plasmid Miniprep I Kit (Zymo Inc. USA). The correct colonies checked by PCR were combined, and subjected to yeast miniprep to get small number of plasmids. The plasmids obtained from yeast miniprep was introduced into Escherichia coli TOP 10 by electroporation. After plasmid extraction from E. coli using Zyppy Plasmid Miniprep Kit (Zymo Research, USA), plasmids were sequenced to confirm identities. Remaining A. nidulans plasmids were generated following the same protocol of pML2001. The resulting recombinant plasmids listed in Table S3. For $A$. nidulans transformation, necessary plasmids were added to $60 \mu \mathrm{L}$ A. nidulans A1145 protoplast suspension prepared above and the mixture was incubated on ice for $60 \mathrm{~min}$. After incubating on ice, $600 \mu \mathrm{L}$ of PEG solution (60\% PEG, $50 \mathrm{mM}$ calcium chloride and $50 \mathrm{mM}$ Tris-HCl, $\mathrm{pH} 7.5$ ) was added to the protoplast mixture, followed by additional incubation at room temperature for $20 \mathrm{~min}$. The mixture was spread on the regeneration medium (CD solid medium with $1.2 \mathrm{M}$ sorbitol and appropriate supplements including $10 \mathrm{mM}$ uridine, $5 \mathrm{mM}$ uracil and/or $0.5 \mu \mathrm{g} / \mathrm{mL}$ pyridoxine $\mathrm{HCl}$ and/or $2.5 \mu \mathrm{g} / \mathrm{mL}$ riboflavin depending on the plasmids being transformed) and incubated at $30{ }^{\circ} \mathrm{C}$ for $2-3$ days until single colonies appear. Isolated transformants were grown in CD-ST media (1 L: $20 \mathrm{~g}$ starch, $20 \mathrm{~g}$ tryptone, $50 \mathrm{~mL} 20 \times$ nitrate salts, $1 \mathrm{~mL}$ trace elements, $\mathrm{pH}$ 6.5) for the production of heterologously expressed metabolites.

\section{Compound identification, isolation, and characterization}

Aspergillus nidulans $\triangle \mathrm{EM}$ transformants were selected for on $\mathrm{CD}$ sorbitol agar ( $2 \%$ glucose as carbon source) appropriately supplemented with riboflavin, uracil, and/or pyridoxine for the set of plasmids introduced. The CDST broth $\left(10 \mathrm{~mL}\right.$ in $50 \mathrm{~mL}$ conical tube) was inoculated with spores and incubated at $28{ }^{\circ} \mathrm{C}$ with shaking at $250 \mathrm{rpm}$ for 3 days. Mycelia were collected and extracted with acetone for $30 \mathrm{~min}$ with sonication. After centrifugation, the supernatant $(200 \mu \mathrm{L})$ was dried down by Speedvac and then re-suspended in methanol $(100 \mu \mathrm{L})$. 
For small scale metabolite analysis: $A$. nidulans $\triangle \mathrm{EM}$ transformants were selected for on $\mathrm{CD}$ sorbitol agar ( $2 \%$ glucose as carbon source) appropriately supplemented with riboflavin, uracil, and/or pyridoxine for the set of plasmids introduced. The CD-ST broth $(10 \mathrm{~mL}$ in $50 \mathrm{~mL}$ conical tube) was inoculated with spores and incubated at $28{ }^{\circ} \mathrm{C}$ with shaking at $250 \mathrm{rpm}$ for 3 days. Mycelia were collected and extracted with acetone for 30 min with sonication. After centrifugation, the supernatant $(200 \mu \mathrm{L})$ was dried down by Speedvac and then re-suspended in methanol $(100 \mu \mathrm{L})$. For small scale analysis of in yeast, $S$. cerevisiae expressing the gene(s) of interest was inoculated in $1 \mathrm{~mL}$ of dropout media for 24 hours. $250 \mu \mathrm{L}$ of starter culture was used to inoculate $1 \mathrm{~mL}$ of YPD. The cells were grown at $28^{\circ} \mathrm{C}, 250 \mathrm{rpm}$ for 48 hours and extracted with an organic phase consisting of $90 \%$ EtOAc and $10 \%$ acetone. The organic phases were dried and dissolved in methanol for analysis. LC-MS analyses were performed on a Shimadzu 2020 EV LC-MS with a reverse-phase column (Phenomenex Kinetex, C18, $1.7 \mu \mathrm{m}, 100$ $\AA$, $2.1 \times 100 \mathrm{~mm}$ ) using positive and negative-mode electrospray ionization with a linear gradient of 5-95\% acetonitrile- $\mathrm{H}_{2} \mathrm{O}$ (containing $0.1 \%$ formic acid) in $15 \mathrm{~min}$ followed by $95 \%$ acetonitrile for $3 \mathrm{~min}$ with a flow rate of $0.3 \mathrm{ml} / \mathrm{min}$.

For large-scale compound purification:

Compound 1. The Aspergillus nidulans $\Delta$ EM expression host co-transformed with plasmids pML2003, pML2004, and pML2005 was selected on CD sorbitol agar. The harvested spores were used to inoculate CD-ST broth. The cultures were in 4 baffled 2L Erlenmeyer flasks containing $1 \mathrm{~L} \mathrm{CD}-\mathrm{ST}$ media at $28^{\circ} \mathrm{C}$ with shaking at $250 \mathrm{rpm}$ with aeration for 4 days. Fungal bodies were separated from supernatant by filtering with miracloth (Millipore). Supernatant was extracted with ethyl acetate $(3 \times)$, fungal bodies, on the other hand, were processed with acetone. The residue after solvent evaporation in vacuo was separated by silica flash chromatography on a CombiFlash instrument with hexane/ethyl acetate solvent system. Fractions determined by LC-MS to contain 1 were pooled and after solvent evaporation, the residue was further separated by semi-preparative RP HPLC using a Shimadzu UFLC system and a COSMOSIL column (Nacalai Tesque Inc., 5C18-MS-II, 20ID x 250mm, flow rate of $4 \mathrm{~mL} / \mathrm{min}$ of solvents A ( $0.1 \%$ formic acid in water) and B (acetonitrile), with an isocratic concentration of $60 \%$ acetonitrile $(\mathrm{MeCN})-40 \%$ water, to afford $1\left(\mathrm{t}_{R} 5.76 \mathrm{~min}\right)$.

Compounds 2,12 and 19. The Aspergillus nidulans $\triangle \mathrm{EM}$ expression host co-transformed with plasmids pML2003, pML2006, and pML2007 was selected on CD sorbitol agar and cultured as described above for compound $\mathbf{1}$. The broth was separated from mycelial mass with miracloth and extracted twice with equal vols. of ethyl acetate, and solvent removed with vacuum. The extract was then subjected to normal-phase CombiFlash purification, using a linear gradient of 30-100\% hexane-EA in $35 \mathrm{~min}$ followed by $100 \%$ EA for $10 \mathrm{~min}$ with a flow rate of $40 \mathrm{~mL} / \mathrm{min}$ (with $40 \mathrm{~g}$ silica column) for initial separation. Fractions containing target compound were combined and further purified with HPLC with a COSMOSIL column (5C18-AR-II, $10 \mathrm{ID} \times 250 \mathrm{~mm}$, flow rate of $4 \mathrm{~mL} / \mathrm{min})$ using an isocratic concentration of $43 \% \mathrm{MeCN}-56 \% \mathrm{H}_{2} \mathrm{O}$, to yield compounds $2\left(\mathrm{t}_{R} 10.9 \mathrm{~min}\right), 12\left(\mathrm{t}_{R} 7.5 \mathrm{~min}\right)$, and $19\left(\mathrm{t}_{R}\right.$ $17.0 \mathrm{~min})$.

Compound 3. The Aspergillus nidulans $\Delta$ EM expression host co-transformed with plasmids pML2003, pML2007, and pML2012 was selected on CD sorbitol agar, and cultured as described above for compound 1. Purification was also done similarly by fractionation over silica flash chromatography followed by semi-preparative RP HPLC (5C18-MS-II, $10 \mathrm{ID} \times 250 \mathrm{~mm}$, flow rate of $4 \mathrm{~mL} / \mathrm{min}$ ), with an isocratic concentration $65 \% \mathrm{MeCN}-35 \% \mathrm{H}_{2} \mathrm{O}$ to yield $3\left(\mathrm{t}_{R} 7.37 \mathrm{~min}\right)$, and then analyzed by HRMS and NMR. 
Compounds 4 and 20. The Aspergillus nidulans $\Delta$ EM expression host co-transformed with plasmids pML2001 and pML2006 was selected on CD sorbitol agar, supplemented with $0.5 \mu \mathrm{g} / \mathrm{mL}$ pyridoxine. The harvested spores were used to inoculate CD-ST broth which was also supplemented with pyridoxine. The cultures were in 8 baffled 2L Erlenmeyer flasks at $28{ }^{\circ} \mathrm{C}$ with shaking at $250 \mathrm{rpm}$ for $96 \mathrm{~h}$. The broth was separated from mycelial and extracted twice with acetone. The residue after solvent evaporation in vacuo was applied to a silica column and separation done by flash chromatography with a gradient of hexanes/acetones as the mobile phase. Fractions determined by LCMS to contain target compound were pooled and after solvent evaporation, the residue was further separated by semi-preparative RP HPLC. Compound $4\left(\mathrm{t}_{R} 13.6 \mathrm{~min}\right)$ and $20\left(\mathrm{t}_{R} 15.0 \mathrm{~min}\right)$ was purified with the Phenomenex Kinetics, $\mathrm{C} 18$ column $(5 \mu \mathrm{m}, 100 \AA, 10 \times 250 \mathrm{~mm})$ using an isocratic wash of $\mathrm{MeCN}-\mathrm{H}_{2} \mathrm{O}(27: 73$, v/v).

Compounds 6, and 9. The Saccharomyces cerevisiae BJ5464-NpgA host harboring plasmid pML2016, pML2023, and pXW55 was selected on uracil, leucine, and tryptophan-dropout SD media. Single colony was picked up from the plate and grown in $2.5 \mathrm{~mL}$ SD uracil-dropout media for 1 day, which was used to inoculate $500 \mathrm{~mL}$ SD uracildropout media and continued to grow 1.5 days. The culture was equally split into 5 portions, each $100 \mathrm{~mL}$ was inoculated into $900 \mathrm{~mL}$ YPD media in shake flasks, and grown at $28{ }^{\circ} \mathrm{C}$ for 3 days with aeration. Supernatant was separated from cell pellet via centrifugation, and was extracted with ethyl acetate twice. The crude extract was separated by silica flash chromatography and the fractions containing target compound were combined. Compound $6\left(\mathrm{t}_{R} 15.5 \mathrm{~min}\right)$ was purified as a broad peak by semipreparative HPLC $\left(\mathrm{MeCN}-\mathrm{H}_{2} \mathrm{O}, 35: 65, \mathrm{v} / \mathrm{v}, 4.0 \mathrm{~mL} / \mathrm{min}\right) \mathrm{using}$ the COSMOSIL column (5C18-AR-II, 10ID $\times 250 \mathrm{~mm})$. The purification of $9\left(\mathrm{t}_{R} 15.8 \mathrm{~min}\right)$ was done by HPLC using a COSMOSIL column $(5 \mathrm{C} 18$-MS-II, $10 \mathrm{ID} \times 250 \mathrm{~mm})$ with an isocratic elution of $25 \%$ of $\mathrm{MeCN}-\mathrm{H}_{2} \mathrm{O}$.

Compound 5. The Aspergillus nidulans $\triangle \mathrm{EM}$ expression host co-transformed with plasmids pML2001, pML2002 and pYTP, and was selected on CD sorbitol agar, and cultured as described above for compound $\mathbf{1}$. The residual was loaded on a normal-phase CombiFlash system and was subject to flash chromatography with a gradient of Hexane/EA (0-15 $\min$ 20\%-70\% EA; 15-40 min 100\% EA). The fractions containing $5\left(\mathrm{t}_{R} 7.4 \mathrm{~min}\right)$ were dried under reduced pressure, and was further applied to a COSMOSIL column with an isocratic wash of $70 \%$ acetonitrile in water supplemented with $0.1 \%$ formic acid.

Compound 7. The Aspergillus nidulans $\triangle \mathrm{EM}$ expression host co-transformed with plasmids pML2001, pML2002 and pML2002, and was selected on CD sorbitol agar. 4 L CD-ST medium supplemented with $0.5 \mu \mathrm{g} / \mathrm{mL}$ pyridoxine were used for fermentation. Using the same initial steps as in that of 1 isolation, the fractions containing $7\left(\mathrm{t}_{R} 7.4\right.$ $\mathrm{min}$ ) was further purified by HPLC using a semi-preparative reversed-phase column with an isocratic elution of $60 \%$ of $\mathrm{MeCN}-\mathrm{H}_{2} \mathrm{O}$.

Compound 8. To confirm DiaC regio- and stereoselectively reduces $\beta$-ketone, large scale $100 \mathrm{ml}$ in vitro reaction was performed. Purified DiaC (4 $\mu \mathrm{M}$ final concentration) was incubated with compound 6 (2 mM), NADPH (4 $\mathrm{mM}$ ) in $50 \mathrm{mM}$ phosphate buffer ( $\mathrm{pH}$ 6.7) for overnight. The reaction mix was extracted with 3 volumes of ethyl acetate, and dried in vacuo. The extract was further purified by HPLC with a semi-preparative reversed-phase column using an isocratic concentration of $25 \% \mathrm{MeCN}-75 \%$ water. Lastly, the compound $8\left(\mathrm{t}_{R} 17.8 \mathrm{~min}\right)$ was further purified by reverse phase HPLC chromatography with a COSMOSIL column (Nacalai Tesque Inc., 5C18MS-II, flow rate $4 \mathrm{~mL} / \mathrm{min}$ ) using an isocratic concentration of $25 \% \mathrm{MeCN}-75 \%$ water. 8 was dissolved in $\mathrm{CD}_{3} \mathrm{OD}$ for NMR analysis. The purified sample also showed the same positive optical rotation with the published data for 8. 
Compounds 10,13 and 14. To obtain compounds 10 and 14 for NMR, large scale $50 \mathrm{ml}$ in vitro reaction contained $2 \mathrm{mM} \mathrm{8}, 4 \mathrm{mM}$ SAM cofactor with $6 \mu \mathrm{M}$ AoiQ (MT) or NgvF in $50 \mathrm{mM}$ phosphate buffer (pH 6.7). For preparation of compound 13, compound 6 was used as substrate to incubate with $6 \mu \mathrm{M} \mathrm{NgvF}$ instead. After incubation at $30^{\circ} \mathrm{C}$ for 6 hours, the reaction mixture was extracted extensively with ethyl acetate, and dried in vacuo, respectively. The residual was loaded on a normal-phase CombiFlash system and was subject to flash chromatography with a gradient of Hexane/EA (0-15 $\min 20 \%-70 \%$ EA; 15-40 min 100\% EA). Fractions containing abundant target compounds were combined, concentrated, and was further purified by reverse-phase HPLC chromatography with a COSMOSIL column (5C18 MS-II, flow rate $4 \mathrm{~mL} / \mathrm{min})$, to yield $10\left(\mathrm{t}_{R} 12.0 \mathrm{~min}\right) \mathrm{using} \mathrm{MeCN}-\mathrm{H}_{2} \mathrm{O}(30: 70, \mathrm{v} / \mathrm{v}), 13\left(\mathrm{t}_{R} 14.1\right.$ $\min )$ using $\mathrm{MeCN}-\mathrm{H}_{2} \mathrm{O}(50: 50, \mathrm{v} / \mathrm{v})$, and 14 ( $\left.\mathrm{t}_{R} 19.8 \mathrm{~min}\right)$ using $\mathrm{MeCN}-\mathrm{H}_{2} \mathrm{O}(32: 68, \mathrm{v} / \mathrm{v})$.

Compound 16. To obtain 16, $20 \mathrm{mg} 15$ was dissolved in acetonitrile and add in $50 \mathrm{mM}$ phosphate buffer (pH 6.7). The production of $\mathbf{1 6}$ was monitored, and then extracted with ethyl acetate. The extract was concentrated in vacuo. The sample was purified by a reverse-phase HPLC with a COSMOSIL column (5C18-AR-II, flow rate $4 \mathrm{~mL} / \mathrm{min}$, $\left.\mathrm{MeCN}-\mathrm{H}_{2} \mathrm{O}, 50: 50\right)$, to afford $\mathbf{1 6}\left(\mathrm{t}_{R} 9.5 \mathrm{~min}\right)$.

Compounds 20, 21, 22 and 23. To produce sufficient amount of compound 20, 21, 22 and 23, 12 liter LB media inoculated with E.coli BL21(DE3) harboring plasmid pML2008 expressing AoiQ (FDH) domain was grown until $\mathrm{OD}_{600}$ reached 1.0.0.1 $\mathrm{mM}$ IPTG was added after the culture was cooled down to $16^{\circ} \mathrm{C}$ to induce protein expression. After induction for 6 hours, 4, 8 or 12 was fed to media with final concentration $3 \mathrm{mg} / \mathrm{L}$, respectively. After 4-day incubation at $28^{\circ} \mathrm{C}$, liquid culture was extracted extensively with ethyl acetate. The residue after solvent evaporation in vacuo was separated by silica flash chromatography on a CombiFlash instrument with hexane/ethyl acetate solvent system. Fractions determined by LC-MS to contain target compounds were pooled and after solvent evaporation, the residue was further separated by semi-preparative RP HPLC using a Shimadzu UFLC system and a COSMOSIL column (5C18-AR-II, 20ID $\times 250 \mathrm{~mm}$, flow rate of $4 \mathrm{~mL} / \mathrm{min}$ ), with an isocratic concentration of mobile phase, to afford 21 ( $\left.\mathrm{t}_{R} 9.73 \mathrm{~min}\right)$ using $\mathrm{MeCN}-\mathrm{H}_{2} \mathrm{O}(45: 55, \mathrm{v} / \mathrm{v}), 22\left(\mathrm{t}_{R} 13.1 \mathrm{~min}\right)$ using $\mathrm{MeCN}-\mathrm{H}_{2} \mathrm{O}(30: 70$, $\mathrm{v} / \mathrm{v})$, and $23\left(\mathrm{t}_{R} 15.3 \mathrm{~min}\right)$ using $\mathrm{MeCN}-\mathrm{H}_{2} \mathrm{O}(30: 70, \mathrm{v} / \mathrm{v}) .20\left(\mathrm{t}_{R} 15.0 \mathrm{~min}\right)$ was purified with the Phenomenex Kinetics, $\mathrm{C} 18$ column $(5 \mu \mathrm{m}, 100 \AA$, $10 \times 250 \mathrm{~mm})$ using $\mathrm{MeCN}-\mathrm{H}_{2} \mathrm{O}(27: 73$, v/v).

\section{Spectroscopic analyses}

NMR spectra were obtained with a Bruker AV500 spectrometer with a $5 \mathrm{~mm}$ dual cryoprobe at the UCLA Molecular Instrumentation Center. ( ${ }^{1} \mathrm{HNMR} 500 \mathrm{MHz},{ }^{13} \mathrm{CNMR} 125 \mathrm{MHz}$ ). High resolution mass spectra were obtained from an Agilent LC-Q-TOF 6545 at the UCLA Molecular Instrumentation Center. Optical rotations were measured on a Rudolph Research Analytical Autopol III Automatic Polarimeter. All LC-MS analyses were performed on a Shimadzu 2020 EVLC-MS (Phenomenex kinetex, $1.7 \mu \mathrm{m}, 2.0 \times 100 \mathrm{~mm}, \mathrm{C} 18$ column) using positive and negative mode electrospray ionization with a linear gradient of 5-95\% $\mathrm{MeCN}-\mathrm{H}_{2} \mathrm{O}$ supplemented with $0.1 \%(\mathrm{v} / \mathrm{v})$ formic acid in $15 \mathrm{~min}$ followed by $95 \% \mathrm{MeCN}$ for $5 \mathrm{~min}$ with a flow rate of $0.3 \mathrm{~mL} / \mathrm{min}$. Control strains carrying empty vectors were similarly analyzed to obtain a differential metabolite analysis profile.

\section{Reconstitution of chlorinated orthosporins (1-3) pathway in $S$. cerevisiae}

The intron free ORFs of diaA, diaB, diaC, AoiQ (FDH-MT), AoiQ (FDH), NgvE, NgvF, and NdlE were amplified by PCR using cDNA from the corresponding $A$. nidulans transformant as a template, and ligated to linear 
expression vector. DiaA gene was inserted into XW02 vector. Genes of DiaB and DiaC were inserted into XW06 vector. AoiQ (FDH-MT), AoiQ (FDH), NgvE, $N g v F$, and $N d l E$ were inserted into XW55 vector. Each plasmid was introduced into BJ5464-NpgA with Frozen E-Z Yeast Transformation Kit (Zymo) selected by dropout media, uracil-dropout for XW55 vector, leucine-dropout for XW02 vector, tryptophan-dropout for XW06 vector selection. Saccharomyces cerevisiae BJ5464-NpgA was transformed with corresponding plasmid combinations and was grown on select nutrient drop-out SD plates at $28{ }^{\circ} \mathrm{C}$ for 3 days. Single colony was picked up and grown in $2 \mathrm{~mL}$ SD media with selective nutrients dropped out for 1.5 days. $0.25 \mathrm{~mL}$ of SD media was then transferred to $1 \mathrm{~mL}$ YPD ( $2 \%$ dextrose) in a glass tube. The culture was shaken at $250 \mathrm{rpm}$ at $28{ }^{\circ} \mathrm{C}$ for $2-3$ days, target compounds are detectable after $48 \mathrm{hrs}$.

\section{Expression and purification of AoiQ (FDH-MT) and dissected AoiQ (FDH) from S. cerevisiae}

AoiQ (FDH-MT) and dissected AoiQ (FDH) were produced in Saccharomyces cerevisiae BJ5464. The intron free ORFs of AoiQ (FDH-MT) and AoiQ (FDH) were amplified by PCR and inserted into XW55 vector with $N$-terminal hexa-histidine tag to give pML2018 and pML2019. The expression plasmid was transformed into BJ5464 with Frozen EZ Yeast Transformation Kit (Zymo) and selected by uracil dropout media. Single colony was picked up from the plate and grown in $2.5 \mathrm{~mL}$ SD uracil-dropout media for 1 day, which was used to inoculate 500 $\mathrm{mL}$ SD uracil-dropout media and continued to grow 1.5 days. The culture was equally split into 5 portions, each $100 \mathrm{~mL}$ was inoculated into $900 \mathrm{~mL}$ YPD media in shake flasks, and grown at $28^{\circ} \mathrm{C}$ for 3 days with aeration. Cells were pelleted, and then fully resuspended in buffer $\left(100 \mathrm{mM} \mathrm{Na}_{2} \mathrm{HPO}_{4}, 150 \mathrm{mM} \mathrm{NaCl}, \mathrm{pH} 7.8\right)$. The cell suspension was supplemented with $50 \mu \mathrm{M}$ flavin adenine dinucleotide (FAD) and lysed by blending and sonication, followed by high-speed centrifugation. The supernatant was incubated with Ni-NTA resin for $1 \mathrm{hr}$, and the mixture was applied to a column for affinity chromatography with gradually increasing concentrations of imidazole in buffer. Pure fractions were identified by SDS-PAGE and were combined and concentrated by Amicon concentrators (Millipore). The concentrated protein samples were buffer-exchanged into buffer containing $50 \mathrm{mM}$ phosphate buffer (pH 8.0), $200 \mathrm{mM} \mathrm{NaCl}$, and $10 \%$ glycerol, and stored at $-80{ }^{\circ} \mathrm{C}$. Protein concentrations were determined by Bradford assay.

\section{Expression and purification of dissected AoiQ (MT), DiaC, NgvF and Fre from E. coli BL21(DE3)}

The intron free ORFs of AoiQ (MT), DiaC, NgvF and Fre were amplified by PCR using cDNA from the corresponding $A$. nidulans transformant as a template, and ligated to linear expression vector pET28a via Gibson Assembly (NEW ENGLAND Biolabs Inc., USA), according to the manufacturer's protocol. The resultant plasmids (Table S3) were sequenced and verified. The plasmids were then transformed into E. coli BL21(DE3) individually and grown overnight in $5 \mathrm{~mL}$ of LB medium with $50 \mu \mathrm{g} / \mathrm{mL}$ kanamycin at $37^{\circ} \mathrm{C}$. The overnight cultures were used as seed cultures for $1 \mathrm{~L}$ fresh LB media containing $50 \mu \mathrm{g} / \mathrm{mL}$ kanamycin and incubated at $37{ }^{\circ} \mathrm{C}$ until the $\mathrm{OD}_{600}$ reached 0.8 . The cultures were cooled on ice, before addition of $0.1 \mathrm{mM}$ isopropyl- $\beta$-D-thiogalactopyranoside (IPTG, GoldBio, USA) to induce protein expression. The expression was performed at $16{ }^{\circ} \mathrm{C}$ for overnight duration. E. coli cells were harvested by centrifugation at $5300 \mathrm{rpm}$ for $15 \mathrm{~min}$ and resuspended in $30 \mathrm{~mL}$ A10 buffer (50 $\mathrm{mM}$ phosphate buffer, $150 \mathrm{mM} \mathrm{NaCl}, 10 \mathrm{mM}$ imidazole, $\mathrm{pH} \mathrm{8.0)} \mathrm{containing} 1$ tablet of PierceTM protease inhibitor (Thermo Scientific). The cell suspension was lysed on ice by sonication and the lysate was centrifuged at 17,000 $\mathrm{g}$ 
for $15 \mathrm{~min}$ at $4{ }^{\circ} \mathrm{C}$ to remove the insoluble cellular debris. The recombinant hexa his-tagged proteins were purified individually from corresponding soluble fractions by affinity chromatography with Ni-NTA agarose resin (Qiagen) according to the manufacturer's instructions. The purified proteins were concentrated and exchanged into storage buffer (50 mM Phosphate buffer, $200 \mathrm{mM} \mathrm{NaCl}, 10 \%$ glycerol, $\mathrm{pH}$ 8.0) with Centriprep filters (Amicon). SDSPAGE was performed to check the protein purity and Bradford Protein Assay (Bio-Rad) was used to calculate protein concentration with bovine serum albumin (BSA, Sigma-Aldrich) as standard. The proteins were aliquoted and stored at $-80{ }^{\circ} \mathrm{C}$ until used in in vitro assays.

\section{Biotransformation assays in $S$. cerevisiae}

To verify the function of enzyme(s) of interest by yeast biotransformation, S. cerevisiae BJ5464 strain transformed with individual plasmids were used for biotransformation. The transformant yeast strains were selected on solid selective drop-out media for 2-3 days, then single colonies were inoculated into $3 \mathrm{~mL}$ selective drop-out media and grown for 24 hours to be used as inoculums for biotransformation in YPD media. $3 \mathrm{~mL}$ YPD media was inoculated with yeast transformants where optical density at $600 \mathrm{~nm}\left(\mathrm{OD}_{600}\right)$ was 0.1 and grown for 24 hours before biosynthetic intermediates were added at concentrations of $500 \mu \mathrm{M}$ each. After the addition of the substrates, the culture was grown for an additional 12 hours at $250 \mathrm{rpm}$ at $28^{\circ} \mathrm{C}$ for 12 hours, before the pellet was extracted with acetone and supernatant with ethyl acetate. The extracts were dried under reduced pressure and dissolved in methanol for analysis in LC-MS as described above.

\section{In vitro assay for DiaC}

$50 \mu \mathrm{L}$ scale in vitro assay of DiaC were performed at $30{ }^{\circ} \mathrm{C}$ for $3 \mathrm{~h}$, in $50 \mathrm{mM}$ Phosphate buffer (pH 6.7), containing $10 \mu \mathrm{M}$ DiaC, $1 \mathrm{mM}$ nicotinamide dinucleotide phosphate hydrogen (NADPH, Cayman Chemicals) or 1 $\mathrm{mM}$ reduced nicotinamide adenine dinucleotide (NADH, Sigma-Aldrich), and $\sim 500 \mu \mathrm{M}$ compound $\mathbf{6}$. The reaction was quenched with equal volume of $\mathrm{MeCN}$ after 3 hours incubation at $30^{\circ} \mathrm{C}$, and centrifuged at $17,000 \mathrm{~g}$ for $5 \mathrm{~min}$ before LC-MS analysis.

\section{In vitro assay for AoiQ (MT) or NgvF}

Assays for AoiQ (MT) or NgvF were carried out in $50 \mathrm{mM}$ phosphate buffer (pH 6.7), containing $6 \mu \mathrm{M}$ enzyme, $\sim 0.5 \mathrm{mM} \mathrm{2,} \mathrm{6,} \mathrm{or} \mathrm{8,} 2 \mathrm{mM} S$-adenosylmethionine (SAM, Sigma Aldrich) in $50 \mu \mathrm{L}$ of solution. The reaction was incubated at $30^{\circ} \mathrm{C}$ for $1.5 \mathrm{hrs}$, quenched with equal volume of $\mathrm{MeCN}$, and centrifuged at $17,000 \mathrm{~g}$ for 5 min before LC-MS analysis.

\section{In vitro assays for AoiQ (FDH)}

In vitro assays of AoiQ (FDH) were performed in $50 \mathrm{mM}$ phosphate buffer ( $\mathrm{pH}$ 6.7). The reaction typically contains $150 \mu \mathrm{M}$ of AoiQ (FDH), $50 \mu \mathrm{M}$ flavin adenine dinucleotide (FAD, Sigma Aldrich), $25 \mathrm{mM} \mathrm{NaCl}, 10 \mathrm{mM}$ $\mathrm{NAD}(\mathrm{P}) \mathrm{H}, 500 \mu \mathrm{M}$ of compounds $\mathbf{2}, \mathbf{4}$, or $\mathbf{1 2}$. After 3 hours at $30^{\circ} \mathrm{C}$, all reactions were quenched with equal volume of $\mathrm{MeCN}$, centrifuged at 17,000 $\mathrm{g}$ for $5 \mathrm{~min}$ before LC-MS analysis.

\section{Coupled in vitro activity assay of AoiQ (FDH), DiaC, and/or AoiQ (MT)}


Coupled in vitro activity assay for AoiQ (FDH) with DiaC together and/or AoiQ (MT) was performed in 100 $\mu \mathrm{L}$ of $50 \mathrm{mM}$ phosphate buffer ( $\mathrm{pH}$ 6.7). The assay contained $5 \mu \mathrm{M}$ DiaC, $50 \mu \mathrm{M}$ FAD, $10 \mathrm{mM} \mathrm{NADH}, 25 \mathrm{mM}$ $\mathrm{NaCl}, 1 \mathrm{mM}$ SAM, $500 \mu \mathrm{M}$ compound 6, and $150 \mu \mathrm{M}$ AoiQ (FDH) and/or $6 \mu \mathrm{M}$ AoiQ (MT). The reaction mixture was incubated at $30^{\circ} \mathrm{C}$ for 3 hours. The reaction was quenched with three volumes of MeCN, centrifuged at 17,000 $g$ for $5 \mathrm{~min}$, and the supernatant was analyzed by LC-MS.

\section{Chemical semi-synthesis of compound 15}

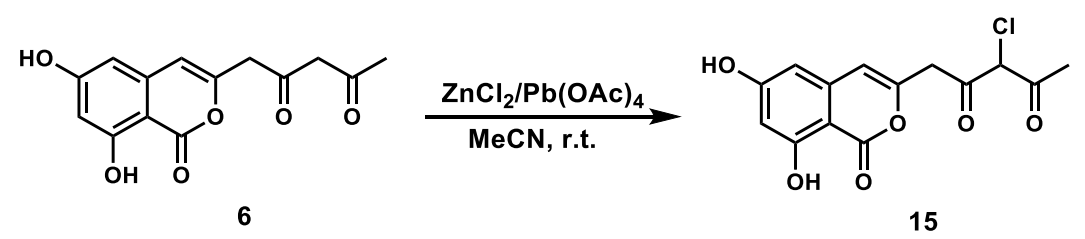

$\mathrm{Pb}(\mathrm{OAc})_{4}$ (TCI America; $2.0 \mathrm{mmol}$ ) was dissolved in $\mathrm{MeCN}\left(20 \mathrm{~mL}\right.$ ). $\mathrm{ZnCl}_{2}$ (fisher scientific; $2.0 \mathrm{mmol}$ ) was added to the $\mathrm{MeCN}$ solution, and the mixture was stirred for $5 \mathrm{~min}$ at room temperature. After adding the compound 6 $\left(2.0 \mathrm{mmol}\right.$ ) to the above solution (substrate $6 / \mathrm{ZnCl}_{2} / \mathrm{Pb}(\mathrm{OAc})_{4}=1: 1: 1$ equiv), ${ }^{[5]}$ the resulting mixture was stirred at room temperature for $12 \mathrm{~h}$. After evaporating the solvent under reduced pressure, the resulting residue was applied to a normal-phase CombiFlash system and was subject to flash chromatography with a gradient of Hexane/EA ( 0 $15 \min 20 \%-70 \% \mathrm{CH}_{2} \mathrm{Cl}_{2} ; 15-40 \min 100 \% \mathrm{CH}_{2} \mathrm{Cl}_{2}$ ). Fractions containing 15 were combined, concentrated, and was further purified by reverse-phase HPLC chromatography using a COSMOSIL column (5C18 MS-II, flow rate $\left.4 \mathrm{~mL} / \mathrm{min}, \mathrm{MeCN}-\mathrm{H}_{2} \mathrm{O}, 50: 50\right)$, to yield $15\left(\mathrm{t}_{R} 11.8 \mathrm{~min}\right)$. Compound 17 was detected as major product when molar ratio of compound $\mathbf{6} / \mathrm{ZnCl}_{2} / \mathrm{Pb}(\mathrm{OAc})_{4}$ was 1:2:2 equiv. However, purification of $\mathbf{1 7}$ was unsuccessful because of its instability.

\section{Sequence information}

AoiQ (FDH) domain amino acid sequence MTNVPEKCQVLVIGGGPAGSYSASALAREGIDVVLLEAEKFPRYHIGESMLPSMRHFLKFIDAYDKWDA HGFNVKNGGAFRLNWSRPETYTDFIAAGGPGGYAWNVIRSEADELLFKHAAECGVKTFDDTKVASIEFA PSEDANPLGRPVSATWTRKDGTSGTLAMDYIVDASGRNGLISTKYLKNRTFNKGLRNVASWGYWKGGG VHGVGTHKEGAPYFEALKDASGWVWFIPLHNGTHSVGVVQNQEMATEKKRKMAEPSSKGFYLESLEFV PGIKDLLSNAELISEVKSASDWSYSASNYAFPGVRIAGDAGSFIDPFFSSGVHLALSGGLSAATTIAAAIRG DCDETVAASWHDKKTSESYTRFLLVVSSALKQIRSQDEPVISDFDEQSFERAFDLFRPIIQGQADADAKGK LTQAEMSKTVEFCFRAFAHVSFEEKEALVQKLKSLGHDADANDEANRKALDELEKHLTPEEQAILKTLK GRRMVRPEDSLNIDNFTLDSIDGLAPRLERGNLGLSPAKKAEVKYTTHDALSFLNGEARAAKKTLSNGES QT

AoiQ (MT) domain amino acid sequence MADLIAAEKISSQTSIEEGTRHRLISSLHQSAEDLETPFDTVVRLVDAGRQTAMVCIGGDLGIFKSLVESKR PLSAEELAKATMADPLLVARIMRYMVASRLVGETGPDQYVASKKTYVFADPRIEHPIRFFHAFSNPAFHAL PEFLKETGYQNEPKGSAFQKALNTDLEPYPWLKQHPDVLKNFQAAMRLTRDANGVDMMPLDQSVSIGH 
DGAMFVDIGGNTGHQAAEVLSKYPELAGRVIVQDRGEVIKCAPDIKGIQWMEHDFFQTQPVKGAKYYY LRAILHNWDDKNTVQILSNIVPAMSADSLVAIDEVVVPEENAHVWPAGLDLQMYSLFSTTERTASQWDAI LDKAGLRAVAVKKYAPVMQSSVIFAAAK

$\underline{\text { NgvF amino acid sequence }}$

MDDLIAPLGAFSHLDEANRLRLQDGLHEAAEALETPYDMMVRLLNAGRQTAMLKIGYDLEIFTTLASRA EPFSVDELASGKSADPLLMSRVLRFLAANRFITETDKNLFTGNKSTKGLSDPRIQGGLLHAFHISVPAYQA LPEYLQENKYQNTTGGQCAWHKSANTDLEFFPWAKQNPQILKYFQQLMSIPRDGSWLDVMPFEDEAND ATDRKVFVDIGGNIGHQCARLLAKHPSLAGRVVLQDLEETIRRAPEVNGVDFMVHDFFTKQPVKGAKFY YLRAILHDWEDDKSVEILKQIIPAMSEDSQILIDDLALPNTGVHWWSTCIDMQMYIMHGAMERTVDQWY GLLDKAGLKVKEIKTYSPVMRNSVIVAIPK

DiaC amino acid sequence

MATPPSYTKTTHTATYAGINPTQPGLSTAGKVVLITGASGGIGRATASSFAASGPRALILLGRRADALAETA TIVRTSHAEVTIQTHEAELCDAASVRNAMNKVAAEFGGIDILVHCAGVLAPVVPLLEADPATFLDGYKTT VVGTLVTAQAVVLANRTVSASEDKPVTFINLTTAGILFPPFPGMGAYVSSKMAAVKLLQSFATENPQVRLH NVHPGLLRTAMSAKLAETIQLPYAYDDITLPADFLVWIASPEAEFLKDKIVFAAWDVDELKARQKEIVGGP PGTGELWLGYQGFPRFIAGQPLSGTQ 


\section{Supplementary Tables}

Table S1. Comparative BLASTp analysis of the dia gene cluster.

\begin{tabular}{|c|c|c|c|c|}
\hline $\begin{array}{l}\text { Aspergillus } \\
\text { oryzae } \\
\text { Dia }\end{array}$ & $\begin{array}{l}\text { Aspergillus nidulans } \\
(\% \text { identity / \% } \\
\text { similarity }) \\
N d l \\
\end{array}$ & $\begin{array}{l}\text { Penicillium nalgiovense } \\
(\% \text { identity / \% } \\
\text { similarity) } \\
\mathrm{Ngv}\end{array}$ & $\begin{array}{l}\text { Trichoderma harzianum } \\
(\% \text { identity / \% } \\
\text { similarity) } \\
\text { Hzn }\end{array}$ & $\begin{array}{l}\text { Predicted } \\
\text { functional } \\
\text { annotation }\end{array}$ \\
\hline DiaA & $\begin{array}{l}\text { XP_664675.1 NdlA (78 } \\
\text { / 85) }\end{array}$ & $\begin{array}{l}\text { OQE96240.1 NgvA (46 / } \\
62)\end{array}$ & $\begin{array}{l}\text { XP_024771862.1 HznA } \\
(68 / 80)\end{array}$ & $\begin{array}{l}\text { NRPKS } \\
\text { (SAT_KS_MAT } \\
\text { _PT_ACP) }\end{array}$ \\
\hline DiaB & \begin{tabular}{|ll}
$\begin{array}{l}\text { XP_664674.1 Nd18B } \\
(74 / 83)\end{array}$ \\
\end{tabular} & $\begin{array}{l}\text { OQE96295.1 NgvB (43/ } \\
59)\end{array}$ & $\begin{array}{l}\text { XP_024771861.1 HznB } \\
(64 / 75)\end{array}$ & Lactamase \\
\hline DiaC & $\begin{array}{l}\text { XP_664678.1 NdlC (87 } \\
\text { / 94) }\end{array}$ & $\begin{array}{l}\text { OQE91382.1 NgvC (88 / } \\
94)\end{array}$ & $\begin{array}{l}\text { XP_024771865.1 HznC } \\
(56 / 66)\end{array}$ & $\begin{array}{l}\text { Short chain } \\
\text { dehydrogenase }\end{array}$ \\
\hline DiaD & $\begin{array}{l}\text { XP_664679.1 NdlD (79 } \\
\text { / 89) }\end{array}$ & $\begin{array}{l}\text { OQE91616.1 NgvD (74 / } \\
84)\end{array}$ & $\begin{array}{l}\text { XP_024771863.1 HznD } \\
(66 / 82)\end{array}$ & $\begin{array}{l}\text { Flavin } \\
\text { oxidoreductase }\end{array}$ \\
\hline \multirow[t]{2}{*}{ AoiQ } & \multirow[t]{2}{*}{$\begin{array}{l}\text { XP_664673.1 NdlE (83 } \\
\text { / 88) }\end{array}$} & $\begin{array}{l}\text { OQE91609.1 NgvE (68 / } \\
80)\end{array}$ & \multirow[t]{2}{*}{$\begin{array}{l}\text { XP_024771860.1 HznE } \\
(74 / 84)\end{array}$} & \multirow{2}{*}{$\begin{array}{l}\text { Flavin } \\
\text { dependent } \\
\text { halogenase- } \\
\text { MT }\end{array}$} \\
\hline & & $\begin{array}{l}\text { OQE91620.1 NgvF (48 / } \\
67)\end{array}$ & & \\
\hline
\end{tabular}


Table S2. Primers used in this study

\begin{tabular}{|c|c|}
\hline Primers & Sequence (5'-3') \\
\hline pML $2001 \mathrm{~F} 1$ & AGCTTCATCCCCAGCATCATTACACCTCAGCAATGGTCAACATTTACATTTTCGGAGATC \\
\hline pML $2001 \mathrm{R} 1$ & TGGAGGACATACCCGTAATTTTCTGGGCATTTAAATCCTGACTGTGTCGATCTCCATTCC \\
\hline pML $2001 \mathrm{~F} 2$ & CGTATGATGAAGGCTGCGGTC \\
\hline pML 2001 R2 & CACACCGTAACACTTGAACCGAC \\
\hline pML 2002 F1 & GACTAACCATTACCCCGCCACATAGACACATCTAAACAATGGAAGAACTGACCCGAAGTC \\
\hline pML 2002 R1 & AAGGGTATCATCGAAAGGGAGTCATCCAATTTAAATACAAAGTGTCTCGGAGTAGAGCTG \\
\hline pML 2003 F1 & GCTTCATCCCCAGCATCATTACACCTCAGCAATGATCGAAAACACCACTATTACTCCTTC \\
\hline pML 2003 R1 & TGGAGGACATACCCGTAATTTTCTGGGCATTTAAATGCCTGAGACCTAAAGTTACCATGG \\
\hline pML 2003 F2 & CTATTTGAGCGAGCACCTTGGTTC \\
\hline pML 2003 R2 & GATGCGATCGAGTTGGAGTACCTG \\
\hline pML 2004 F1 & CTAACCATTACCCCGCCACATAGACACATCTAAACAATGCTTTCAACAACCATGCCG \\
\hline pML 2004 R1 & GGTATCATCGAAAGGGAGTCATCCAATTTAAATGTCTACGTAGTTTCCAGCTGACTTCTC \\
\hline pML 2005 F1 & ССТTCTCTGAACAATAAACCCCACAGAAGGCATTTATGACGAACGTTCCGGAAAAGTG \\
\hline pML 2005 R1 & TGAGACCCAACAACCATGATACCAGGGGATTTAAATCGCAGATTTGATCCGAGCGAATTG \\
\hline pML 2006 F1 & CCATTACCCCGCCACATAGACACATCTAAACAATGACGAACGTTCCGGAAAAGTG \\
\hline pML 2006 R1 & AAGCTGTTTGATGATTTCAGTAACGTTAAGTGGTCAGGTTTGGGATTCGCCATTTGAAAG \\
\hline pML 2006 F2 & CCACTTAACGTTACTGAAATCATCAAACAG \\
\hline pML 2006 R2 & CTAAAGGGTATCATCGAAAGGGAGTCATCCAAAGAAGGATTACCTCTAAACAAGTGTACC \\
\hline pML 2007 F1 & CTAACCATTACCCCGCCACATAGACACATCTAAACAATGCTTTCAACAACCATGCCG \\
\hline pML 2007 R1 & GATGAGACCCAACAACCATGATACCAGGGGATTTAAATGTCTACGTAGTTTCCAGCTGAC \\
\hline pML 2008 F1 & CCATTACCCCGCCACATAGACACATCTAAACAATGCTACGTCTCACTCTATTGATCG \\
\hline pML 2008 F2 & AGTTCAAGGATTGGATCAAGCAGTATTGTTAGCCACTTAACGTTACTGAAATCATCA \\
\hline pML 2008 R1 & CTAACAATACTGCTTGATCCAATCCTTGAAC \\
\hline pML 2008 R2 & AACACAGTGGAGGACATACCCGTAATTTTCTGAAGAAGGATTACCTCTAAACAAGTGTAC \\
\hline pML 2009 F1 & CCATTACCCCGCCACATAGACACATCTAAACAATGTTCACTCCAAAAGTATGGACCC \\
\hline pML 2009 R1 & GAGACCCAACAACCATGATACCAGGGGATTTAAATCCTGGAACGATCAATAGAGTGAGAC \\
\hline pML $2010 \mathrm{~F} 1$ & CTAACCATTACCCCGCCACATAGACACATCTAAACAATGGCCGCTATACAGACTGTTC \\
\hline pML 2010 R1 & CTTATACATGATCTAACAACTTCTAGTAAACCGCAATCATGGACGATCTCATCGCTCCC \\
\hline pML 2010 F2 & CTAAAGGGTATCATCGAAAGGGAGTCATCCAATTTAAATGGAAGGCTCCGAAATGGAACC \\
\hline pML 2010 R2 & CCCAATATTCCAACCTTGGGAAGCCCTGGACGAATCTCGGTACTTCTTTCCAATGAGCGC \\
\hline pML $2011 \mathrm{~F} 1$ & CTAACCATTACCCCGCCACATAGACACATCTAAACAATGACTCCGTCTGCATCCCC \\
\hline pML 2011 R1 & CAATATTCCAACCTTGGGAAGCCCTGGACGAATCCTTTTCTTCGTAAGCAGCTACACTCC \\
\hline pML 2011 F2 & TATACATGATCTAACAACTTCTAGTAAACCGCAATCATGTCTGGGGGATTTTACTCGTCG \\
\hline pML 2011 R2 & ACACAGTGGAGGACATACCCGTAATTTTCTGATTTAAATCACGAAGCGAAGGTGAAGAAG \\
\hline pML $2011 \mathrm{~F} 3$ & GTTTCGCAACCAGGTTCTCCAAC \\
\hline pML 2011 R3 & GGCTACGGTGACCAGTATTGATGG \\
\hline
\end{tabular}


Table S2 (continued). Primers used in this study.

\begin{tabular}{|c|c|}
\hline Primers & Sequence (5'-3') \\
\hline pML 2012 F1 & ACTAACCATTACCCCGCCACATAGACACATCTAAACAATGATGAACGTCCCGGAAAAGTG \\
\hline pML 2012 R1 & TAAAGGGTATCATCGAAAGGGAGTCATCCAATTTAAATGAAGACGGGGGTAGTTGTTTCG \\
\hline pML 2013 F1 & CTAACCATTACCCCGCCACATAGACACATCTAAACAATGAATGAAAACGCCAACACTCCC \\
\hline pML 2013 R1 & AACACAGTGGAGGACATACCCGTAATTTTCTGGACATCTATCCAGTGATAAGGTCTCTCG \\
\hline pML 2013 F2 & AAAATGTCGATGCACATGCAAAACAC \\
\hline pML 2013 R2 & CAACAGCTCTCTGCTTGGCTG \\
\hline pML 2014 F1 & CTAACCATTACCCCGCCACATAGACACATCTAAACAATGCCTTCAACCACCAAACCC \\
\hline pML 2014 R1 & TGAGACCCAACAACCATGATACCAGGGGATTTAAATCTCTGAGAAGGAGATGGAGATGGG \\
\hline pML 2015 F1 & CTAACCATTACCCCGCCACATAGACACATCTAAACAATGGCCACCATCCCGCAA \\
\hline pML 2015 R1 & AAGGGTATCATCGAAAGGGAGTCATCCAATTTAAATCTCTCATTCAACTAGTCTTGGCGG \\
\hline pML 2016 F1 & CAACTATCAACTATTAACTATATCGTAATACATGATCGAAAACACCACTATTACTCCTTC \\
\hline pML 2016 R1 & TAGGCATACTTGATAATGAAAACTATAAATCGTTACTTGCTCAACCACGTAACCAAATCC \\
\hline pML 2016 F2 & GAGCTTCATGGCACAGGAACTCAG \\
\hline pML 2016 R2 & GTACAGACTCAGACTCAACAGCGTC \\
\hline pML 2017 F1 & CCAAAGTAGTAAAAGCACATAAAAGAATTAAGAAAATGCCGGAGGGATTTTACTCGTC \\
\hline pML 2017 R1 & TGGAAACTATAAATCGTGAAGGCATGTTTCTAAAGTGGCTGGACCGTTGC \\
\hline pML 2017 F2 & TACAATCAACTATCAACTATTAACTATATCGTAATATGGCCACACCACCATCGTAC \\
\hline pML 2017 R2 & AAAAAAAAAAAATCTTTGACTATTCAATCATTGCGCCTACTGGGTTCCTGACAAAGGC \\
\hline pML 2018 F1 & GGCTAGCCATCACCATCACCATCACCATCACACTAGTATGACGAACGTTCCGGAAAAGTG \\
\hline pML 2018 R1 & TACTTGATAATGAAAACTATAAATCGTGAAGGCATTCACTTGGCAGCCGCAAAG \\
\hline pML 2019 F1 & GCCATCACCATCACCATCACCATCACACTAGTATGACGAACGTTCCGGAAAAGTG \\
\hline pML 2019 R1 & CTTGATAATGAAAACTATAAATCGTGAAGGCATTCAGGTTTGGGATTCGCCATTTGAAAG \\
\hline pML $2020 \mathrm{~F} 1$ & CAAAGTAGTAAAAGCACATAAAAGAATTAAGAAAATGGACGATCTCATCGCTCC \\
\hline pML 2020 R1 & AAAAAAAAAAATCTTTGACTATTCAATCATTGCGCTCAAGTTGCGAACTCCGCAAC \\
\hline pML $2020 \mathrm{~F} 2$ & CAATCAACTATCAACTATTAACTATATCGTAATACATGGCCGCTATACAGACTGTTC \\
\hline pML 2020 R2 & ACTTGATAATGAAAACTATAAATCGTGAAGGCATTTATTTCGGAATGGCCACAATAACCG \\
\hline pML $2021 \mathrm{~F} 1$ & GCTAGCCATCACCATCACCATCACCATCACACTAGTATGGCCGCTATACAGACTGTTCC \\
\hline pML 2021 R1 & TACTTGATAATGAAAACTATAAATCGTGAAGGCATTCAAGTTGCGAACTCCGCAAC \\
\hline pML 2022 F1 & TAGCCATCACCATCACCATCACCATCACACTAGTATGATGAACGTCCCGGAAAAGTG \\
\hline pML 2022 R1 & TACTTGATAATGAAAACTATAAATCGTGAAGGCATCTACTTGGCAGCCGCAAAAATG \\
\hline pML 2023 F1 & CAATCAACTATCAACTATTAACTATATCGTAATATGCCGGAGGGATTTTACTCG \\
\hline pML 2023 R1 & TGGAAACTATAAATCGTGAAGGCATGTTTCTAAAGTGGCTGGACCGTTGC \\
\hline pML 2024 F1 & CATCATCACAGCAGCGGCCTGGTGCCGCGCGGCAGCATGGCCACACCACCATCGTAC \\
\hline pML 2024 R1 & CGGATCTCAGTGGTGGTGGTGGTGGTGCTCGAGTCACTACTGGGTTCCTGACAAAGGC \\
\hline pML 2025 F1 & GCCATCACCATCACCATCACCATCACACTAGTATGACGAACGTTCCGGAAAAGTG \\
\hline pML 2025 R1 & CTTGATAATGAAAACTATAAATCGTGAAGGCATTCAGGTTTGGGATTCGCCATTTGAAAG \\
\hline pML 2026 F2 & GTTTTAATGTAGCCAACGGAGGTGC \\
\hline
\end{tabular}


Table S2 (continued). Primers used in this study.

\begin{tabular}{ll}
\hline Primers & Sequence (5'-3') \\
pML 2026 R2 & GCACCTCCGTTGGCTACATTAAAAC \\
pML 2026 F1 & GATCTCAGTGGTGGTGGTGGTGGTGCTCGAGTCATCACTTGGCAGCCGCAAAG \\
pML 2026 R1 & ATCATCACAGCAGCGGCCTGGTGCCGCGCGGCAGCATGGCAGACCTCATTGCAGC \\
pML 2027 F1 & CATCATCACAGCAGCGGCCTGGTGCCGCGCGGCAGCATGGACGATCTCATCGCTCCC \\
pML 2027 R1 & GATCTCAGTGGTGGTGGTGGTGGTGCTCGAGTCATTATTTCGGAATGGCCACAATAACCG \\
pML 2028 F1 & CATCACAGCAGCGGCCTGGTGCCGCGCGGCAGCATGACGAACGTTCCGGAAAAGTG \\
pML 2028 R1 & GGATCTCAGTGGTGGTGGTGGTGGTGCTCGAGTCAGGTTTGGGATTCGCCATTTGAAAGC \\
pML 2029 F1 & TACTTCCAATCCAATGCAATGACAACCTTAAGCTGTAAAGTGACC \\
pML 2029 R1 & TTATCCACTTCCAATGTTATTATCAGATAAATGCAAACGCATCGC \\
pML 2030 F1 & CATCACAGCAGCGGCCTGGTGCCGCGCGGCAGCATGACGAACGTTCCGGAAAAGTG \\
pML 2030 R1 & GGATCTCAGTGGTGGTGGTGGTGGTGCTCGAGTCAGGTTTGGGATTCGCCATTTGAAAGC \\
pML 2030 F2 & GTTTTAATGTAGCCAACGGAGGTGC \\
pML 2030 R2 & GCACCTCCGTTGGCTACATTAAAAC \\
pYTR gpdAp F & GCGGGTGTTCTTGACGATGGCATCCTGCGGCCGCACTCCGGTGAATTGATTTGGGTGAC \\
pYTU gpdAp F & CCTCGCGGGTGTTCTTGACGATGGCATCCTGCACTCCGGTGAATTGATTTGGGTG \\
pYTP gpdAp F & AGTAACCTCGCGGGTGTTCTTGACGATGGCATCCTACTCCGGTGAATTGATTTGGGTG \\
gpdAp R & TGTTTAGATGTGTCTATGTGGCGGG \\
pegpdAp F & GATTCGTCCAGGGCTTCCC \\
pegpdAp R & GATTGCGGTTTACTAGAAGTTGTTAGATCATG \\
CPS1t F & GCGCAATGATTGAATAGTCAAAGATT \\
MLS1p R & TTTCTTAATTCTTTTATGTGCTTTTACTACTTTGTTTAGTTC \\
\hline
\end{tabular}


Table S3. Plasmids used in this study

\begin{tabular}{|c|c|c|}
\hline Plasmids & Vector & Genes \\
\hline pML 2001 & pYTU & aoiG (NRPKS) \\
\hline pML 2002 & pYTR & $\operatorname{aoiF}(\mathrm{MT})$ \\
\hline pML 2003 & pYTU & $\operatorname{diaA}(\mathrm{NRPKS})$ \\
\hline pML 2004 & pYTR & $\operatorname{diaB}$ (Lactamase) \\
\hline pML 2005 & pYTP & $\operatorname{aoiQ}(\mathrm{FDH}-\mathrm{MT})$ \\
\hline pML 2006 & pYTR & $\operatorname{aoiQ}(\mathrm{FDH})$ \\
\hline pML 2007 & pYTP & $\operatorname{dia} B$ (Lactamase) \\
\hline pML 2008 & pYTU & $n g v A(\mathrm{NRPKS})$ \\
\hline pML 2009 & pYTR & $n g v B$ (Lactamase) \\
\hline pML 2010 & pYTP & $n g v E(\mathrm{FDH})-n g v F(\mathrm{MT})$ \\
\hline pML 2011 & pYTU & $n d l A$ (NRPKS)- $n d l B$ (Lactamase) \\
\hline pML 2012 & pYTR & $n d l E(\mathrm{FDH}-\mathrm{MT})$ \\
\hline pML 2013 & pYTU & $h z n A$ (NRPKS) \\
\hline pML 2014 & pYTP & $h z n B$ (Lactamase) \\
\hline pML 2015 & pYTR & $h z n E$ (FDH-MT) \\
\hline pML 2016 & pXW02 & $\operatorname{dia} A(\mathrm{NRPKS})$ \\
\hline pML 2017 & pXW06 & $\operatorname{dia} C$ (SDR)-diaB (Lactamase) \\
\hline pML 2018 & pXW55 & aoiQ (FDH-MT) \\
\hline pML 2019 & pXW55 & $\operatorname{aoiQ}(\mathrm{FDH})$ \\
\hline pML 2020 & pXW55 & $n g v E(\mathrm{FDH})-n g v F(\mathrm{MT})$ \\
\hline pML 2021 & pXW55 & $n g v E(\mathrm{FDH})$ \\
\hline pML 2022 & pXW55 & $n d l E(\mathrm{FDH}-\mathrm{MT})$ \\
\hline pML 2023 & pXW06 & diaB (Lactamase) \\
\hline pML 2024 & pXW55 & $\operatorname{aoi} Q(\mathrm{FDH})-\mathrm{K} 75 \mathrm{~A}$ \\
\hline pML 2025 & pET28a & $\operatorname{dia} C(\mathrm{SDR})$ \\
\hline pML 2026 & pET28a & $\operatorname{aoiQ}(\mathrm{MT})$ \\
\hline pML 2027 & pET28a & $n g v F(\mathrm{MT})$ \\
\hline pML 2028 & pET28a & $\operatorname{aoiQ}(\mathrm{FDH})$ \\
\hline pML 2029 & pET28a & Fre \\
\hline pML 2030 & $\mathrm{pET} 28 \mathrm{a}$ & $\operatorname{aoi} Q(\mathrm{FDH})-\mathrm{K} 75 \mathrm{~A}$ \\
\hline
\end{tabular}


Table S4. Spectroscopic data of compound 8-methyldichlorodiaporthin (1)<smiles>COC1=CC2=CC(C[C@@H](O)C(Cl)Cl)=CC2=C(OC)C1</smiles>

\section{8-methyldichlorodiaporthin (1)}

${ }^{1} \mathrm{H}$ NMR spectrum (500 MHz), ${ }^{13} \mathrm{C}$ NMR spectrum (125 MHz), DMSO- $d_{6}$

\begin{tabular}{|c|c|c|c|}
\hline \multirow[b]{2}{*}{ Position } & \multicolumn{2}{|c|}{$\begin{array}{l}\text { 8-methyldichlorodiaporthin (1) in } \\
\text { DMSO- } d_{6}\end{array}$} & \multirow{2}{*}{$\begin{array}{c}\text { Reported } 1 \text { in DMSO- } d_{6}{ }^{[6]} \\
\delta_{\mathrm{C}} \text {, type }\end{array}$} \\
\hline & $\delta_{\mathrm{H}}(J$ in $\mathrm{Hz})$ & $\delta_{\mathrm{C}}$, type & \\
\hline 1 & & 157.7, C & 157.7, C \\
\hline 3 & & $154.4, \mathrm{C}$ & $154.4, \mathrm{C}$ \\
\hline 4 & $6.44, \mathrm{~d}(2.3)$ & $105.2, \mathrm{CH}$ & $105.2, \mathrm{CH}$ \\
\hline $4 a$ & & 141.7, C & 141.7, C \\
\hline 5 & $6.62, \mathrm{~d}(2.2)$ & $100.5, \mathrm{CH}$ & $100.5, \mathrm{CH}$ \\
\hline 6 & & 165.2, C & 165.2, C \\
\hline 7 & $6.58, \mathrm{~d}(2.3)$ & $98.4, \mathrm{CH}$ & $98.5, \mathrm{CH}$ \\
\hline 8 & & $162.8, \mathrm{C}$ & $162.8, \mathrm{C}$ \\
\hline $8 a$ & & 102.0, C & 102.0, C \\
\hline 9 & $\begin{array}{l}2.57, \mathrm{dd}(9.5,14.5) \\
2.82 \mathrm{dd}(3.2,14.5)\end{array}$ & $36.4, \mathrm{CH}_{2}$ & $36.4, \mathrm{CH}_{2}$ \\
\hline 10 & $4.16, \mathrm{~m}$ & $72.2, \mathrm{CH}$ & $72.2, \mathrm{CH}$ \\
\hline 11 & $6.26, d(3.1)$ & 77.1, CH & $77.1, \mathrm{CH}$ \\
\hline 6-OMe & $3.86, \mathrm{~s}$ & $55.8, \mathrm{CH}_{3}$ & $55.8, \mathrm{CH}_{3}$ \\
\hline 8-OMe & $3.85, \mathrm{~s}$ & $56.1, \mathrm{CH}_{3}$ & $56.1, \mathrm{CH}_{3}$ \\
\hline $10-\mathrm{OH}$ & 6.08 & & \\
\hline
\end{tabular}

NMR spectrum (500 MHz) for ${ }^{1} \mathrm{H}$, NMR spectrum (125 MHz) for ${ }^{13} \mathrm{C}, \mathrm{DMSO}-d_{6}$, "m" means overlapped or multiple with other signals. Chemical shifts are reported in ppm.

HRMS (ESI, $\mathrm{M}+\mathrm{H}^{+}$) $\mathrm{m} / z$ calculated for $\mathrm{C}_{14} \mathrm{H}_{15} \mathrm{Cl}_{2} \mathrm{O}_{5}{ }^{+}$333.0291; found 333.0313 .

$[\alpha]_{\mathrm{D}}^{24.1}+46^{\circ}(c 0.1, \mathrm{MeOH})$. Compound 1 showed the same positive optical rotation with reported dichlorodiaporthin. ${ }^{[7]}$ 
Table S5. Spectroscopic data of compound desmethyldichlorodiaportin (2)

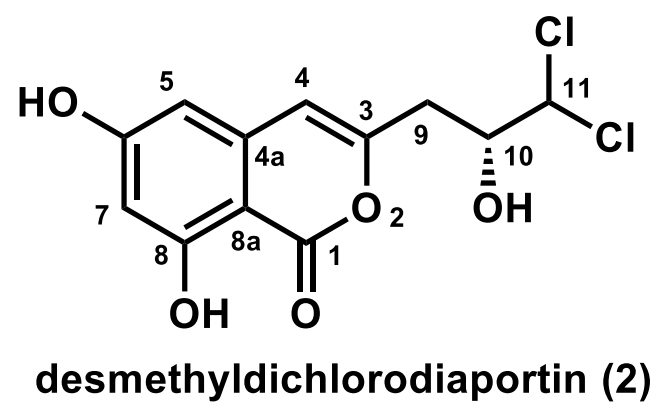

\begin{tabular}{|c|c|c|c|}
\hline \multirow[b]{2}{*}{ Position } & \multicolumn{2}{|c|}{$\begin{array}{l}\text { Desmethyldichlorodiaportin (2) in } \\
\qquad \mathrm{CD}_{3} \mathrm{OD}(500 \mathrm{MHz})\end{array}$} & \multirow{2}{*}{$\begin{array}{l}\text { Reported desmethyldichlorodiaportin (2) in } \\
\qquad \mathrm{CD}_{3} \mathrm{OD}(500 \mathrm{MHz})^{[8]} \\
\delta_{\mathrm{H}}(J \text { in } \mathrm{Hz})\end{array}$} \\
\hline & $\delta_{\mathrm{H}}(J$ in $\mathrm{Hz})$ & $\delta_{\mathrm{C}}$, type & \\
\hline 1 & & 167.6, C & \\
\hline 3 & & $154.6, \mathrm{C}$ & \\
\hline 4 & $6.41, \mathrm{~s}$ & 107.7, CH & $6.41, \mathrm{~s}$ \\
\hline $4 a$ & & $140.9, \mathrm{C}$ & \\
\hline 5 & $6.31, \mathrm{~m}$ & 103.9, $\mathrm{CH}$ & $6.30, \mathrm{~s}$ \\
\hline 6 & & $167.4, \mathrm{C}$ & \\
\hline 7 & $6.31, \mathrm{~m}$ & $102.8, \mathrm{CH}$ & $6.30, \mathrm{~s}$ \\
\hline 8 & & $164.8, \mathrm{C}$ & \\
\hline $8 a$ & & $99.7, \mathrm{C}$ & \\
\hline \multirow[t]{2}{*}{9} & $2.73, \mathrm{dd}(9.6,14.8)$ & $37.3, \mathrm{CH}_{2}$ & $2.72, \mathrm{dd}(9.4,14.8)$ \\
\hline & $2.97, \mathrm{dd}(2.4,14.7)$ & & $2.96, \mathrm{dd}(3.1,14.8)$ \\
\hline 10 & $4.30, \mathrm{dt}(3.3,9.5)$ & $74.2, \mathrm{CH}$ & 4.29, ddd $(3.1,3.4,9.5)$ \\
\hline 11 & $6.02, \mathrm{~d}(3.5)$ & $77.2, \mathrm{CH}$ & $6.02, \mathrm{~d}(3.4)$ \\
\hline
\end{tabular}

NMR spectrum (500 MHz) for ${ }^{1} \mathrm{H}$, NMR spectrum $(125 \mathrm{MHz})$ for ${ }^{13} \mathrm{C}, \mathrm{CD}_{3} \mathrm{OD}$, “m” means overlapped or multiple with other signals. Chemical shifts are reported in ppm.

HRMS (ESI, $\left.\mathrm{M}+\mathrm{H}^{+}\right) \mathrm{m} / z$ calculated for $\mathrm{C}_{12} \mathrm{H}_{11} \mathrm{Cl}_{2} \mathrm{O}_{5}{ }^{+}$304.9978; found 304.9983 .

$$
[\alpha]_{\mathrm{D}}^{24.1}+22^{\circ}(c 0.1, \mathrm{MeOH}) \text {. }
$$

Compound 2 showed the same positive optical rotation with reported dichlorodiaporthin. ${ }^{[7]}$ 
Table S6. Spectroscopic data of compound dichlorodiaporthin (3)

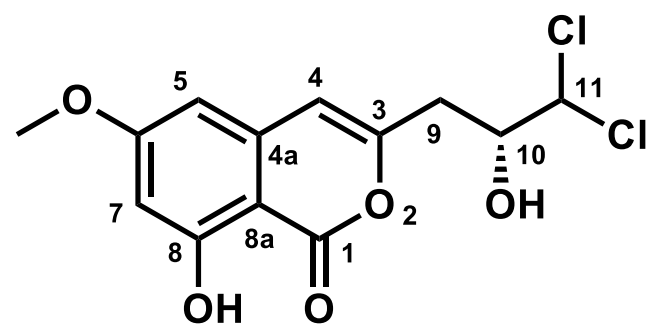

dichlorodiaporthin (3)

\begin{tabular}{|c|c|c|c|c|}
\hline \multirow[b]{2}{*}{ Position } & \multicolumn{2}{|c|}{$\begin{array}{c}\text { Dichlorodiaportin }(3) \text { in }\left(\mathrm{CD}_{3}\right)_{2} \mathrm{CO} \\
(500 \mathrm{MHz})\end{array}$} & \multicolumn{2}{|c|}{$\begin{array}{l}\text { Reported dichlorodiaportin }(3) \text { in }\left(\mathrm{CD}_{3}\right)_{2} \mathrm{CO} \\
(500 \mathrm{MHz})^{[7]}\end{array}$} \\
\hline & $\delta_{\mathrm{H}}(J$ in $\mathrm{Hz})$ & $\delta_{\mathrm{C}}$, type & $\delta_{\mathrm{H}}(J$ in $\mathrm{Hz})$ & $\delta_{\mathrm{C}}$, type \\
\hline 1 & & $166.8, \mathrm{C}$ & & $166.8, \mathrm{C}$ \\
\hline 3 & & $154.7, \mathrm{C}$ & & $154.9, \mathrm{C}$ \\
\hline 4 & $6.57, \mathrm{~s}$ & 107.4, CH & $6.57, \mathrm{~s}$ & $107.4, \mathrm{CH}$ \\
\hline $4 a$ & & $140.4, \mathrm{C}$ & & $140.4, \mathrm{C}$ \\
\hline 5 & $6.54, \mathrm{~d}(2.4)$ & $102.1, \mathrm{CH}$ & $6.54, \mathrm{~d}(2.2)$ & 102.1, CH \\
\hline 6 & & 167.9, C & & 167.9, C \\
\hline 7 & $6.46, \mathrm{~d}(2.3)$ & 101.2, CH & $6.47, \mathrm{~d}(2.2)$ & 101.2, $\mathrm{CH}$ \\
\hline 8 & & 164.3, C & & 164.3, C \\
\hline $8 a$ & & $100.6, \mathrm{C}$ & & $100.6, \mathrm{C}$ \\
\hline \multirow[t]{2}{*}{9} & $2.79, \mathrm{dd}(9.3,14.6)$ & $37.3, \mathrm{CH}_{2}$ & $2.80, \mathrm{dd}(9.2,14.7)$ & $37.3, \mathrm{CH}_{2}$ \\
\hline & $3.01, \mathrm{dd}(3.4,14.6)$ & & $3.02, \mathrm{dd}(3.3,14.7)$ & \\
\hline 10 & $4.40, \mathrm{dt}(3.4,9.3)$ & $73.9, \mathrm{CH}$ & $4.41, \mathrm{~m}$ & 73.9, $\mathrm{CH}$ \\
\hline 11 & $6.19, \mathrm{~d}(3.4)$ & 77.2, $\mathrm{CH}$ & $6.20, \mathrm{~d}(3.3)$ & 77.2, $\mathrm{CH}$ \\
\hline $\mathrm{OMe}$ & $3.90, \mathrm{~s}$ & $56.3, \mathrm{CH}_{3}$ & $3.90, \mathrm{~s}$ & $56.3, \mathrm{CH}_{3}$ \\
\hline $8-\mathrm{OH}$ & $11.08, \mathrm{~s}$ & & $11.08, \mathrm{~s}$ & \\
\hline $10-\mathrm{OH}$ & $5.23, \mathrm{br}, \mathrm{s}$ & & $5.25, \mathrm{br}, \mathrm{s}$ & \\
\hline
\end{tabular}

NMR spectrum $(500 \mathrm{MHz})$ for ${ }^{1} \mathrm{H}$, NMR spectrum $(125 \mathrm{MHz})$ for ${ }^{13} \mathrm{C},\left(\mathrm{CD}_{3}\right)_{2} \mathrm{CO}$, “m” means overlapped or multiple with other signals. Chemical shifts are reported in ppm.

HRMS (ESI, $\mathrm{M}+\mathrm{H}^{+}$) $\mathrm{m} / z$ calculated for $\mathrm{C}_{13} \mathrm{H}_{13} \mathrm{Cl}_{2} \mathrm{O}_{5}{ }^{+} 319.0135$; found 319.0191 .

$$
[\alpha]_{\mathrm{D}}^{24.1}+18.7^{\circ}(c 0.1, \mathrm{MeOH})
$$


Table S7. Spectroscopic data of compound orthosporin (4)

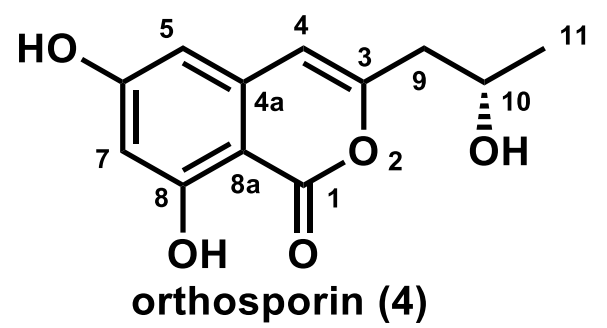

\begin{tabular}{|c|c|c|c|c|}
\hline \multirow[b]{2}{*}{ Position } & \multicolumn{2}{|c|}{$\begin{array}{l}\text { Orthosporin }(4) \text { in }\left(\mathrm{CD}_{3}\right)_{2} \mathrm{CO} \\
(500 \mathrm{MHz})\end{array}$} & \multicolumn{2}{|c|}{$\begin{array}{l}\text { Reported orthosporin }(4) \text { in }\left(\mathrm{CD}_{3}\right)_{2} \mathrm{CO} \\
\qquad(400 \mathrm{MHz})^{[9]}\end{array}$} \\
\hline & $\delta_{\mathrm{H}}(J$ in $\mathrm{Hz})$ & $\delta_{\mathrm{C}}$, type & $\delta_{\mathrm{H}}(J$ in $\mathrm{Hz})$ & $\delta_{\mathrm{C}}$, type \\
\hline 1 & & 167.5, C & & 167.1, C \\
\hline 3 & & 156.2, C & & 156.3, C \\
\hline 4 & $6.36, \mathrm{~m}$ & $140.8, \mathrm{CH}$ & $6.37, \mathrm{~d}(2.1)$ & $140.9, \mathrm{CH}$ \\
\hline $4 a$ & & 103.7, C & & 103.6, C \\
\hline 5 & $6.39, \mathrm{~m}$ & $99.3, \mathrm{CH}$ & $6.40, \mathrm{~d}(2.1)$ & $99.5, \mathrm{CH}$ \\
\hline 6 & & $164.5, \mathrm{C}$ & & $164.4, \mathrm{C}$ \\
\hline 7 & $6.39, \mathrm{~m}$ & $102.5, \mathrm{CH}$ & $6.40, \mathrm{~d}(2.1)$ & $102.4, \mathrm{CH}$ \\
\hline 8 & & 167.1, C & & 167.0, C \\
\hline $8 a$ & & 106.4, C & & 106.4, C \\
\hline 9 & $2.57, \mathrm{~m}$ & $43.9, \mathrm{CH}_{2}$ & $2.58, \mathrm{~m}$ & $43.9, \mathrm{CH}_{2}$ \\
\hline 10 & $4.15, \mathrm{~m}$ & $65.5, \mathrm{CH}$ & $4.16, \mathrm{~m}$ & $65.4, \mathrm{CH}$ \\
\hline 11 & $1.23, \mathrm{~d}(6.1)$ & $23.6, \mathrm{CH}_{3}$ & $1.23, \mathrm{~d}(6.2)$ & $23.6, \mathrm{CH}_{3}$ \\
\hline
\end{tabular}

NMR spectrum (500 MHz) for ${ }^{1} \mathrm{H}$, NMR spectrum $(125 \mathrm{MHz})$ for ${ }^{13} \mathrm{C},\left(\mathrm{CD}_{3}\right)_{2} \mathrm{CO}$, "m" means overlapped or multiple with other signals. Chemical shifts are reported in ppm.

HRMS (ESI, $\mathrm{M}+\mathrm{H}^{+}$) m/z calculated for $\mathrm{C}_{12} \mathrm{H}_{13} \mathrm{O}_{5}{ }^{+}$237.0757; found 237.0757.

$$
[\alpha]_{\mathrm{D}}^{24.1}+34^{\mathrm{o}}(c 0.1, \mathrm{MeOH}) \text {. }
$$


Table S8. Spectroscopic data of compound diaporthin (5)

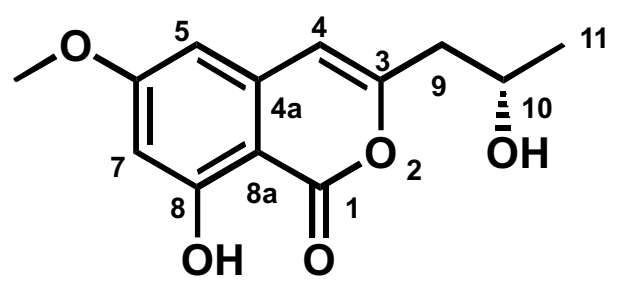

diaporthin (5)

\begin{tabular}{|c|c|c|c|c|}
\hline \multirow[b]{2}{*}{ Position } & \multicolumn{2}{|c|}{$\begin{array}{l}\text { Diaporthin (5) in } \mathrm{CDCl}_{3} \\
(500 \mathrm{MHz})\end{array}$} & \multicolumn{2}{|c|}{$\begin{array}{l}\text { Reported diaporthin (5) in } \mathrm{CDCl}_{3} \\
(400 \mathrm{MHz})^{[10]}\end{array}$} \\
\hline & $\delta_{\mathrm{H}}(J$ in $\mathrm{Hz})$ & $\delta_{\mathrm{C}}$, type & $\delta_{\mathrm{H}}(J$ in $\mathrm{Hz})$ & $\delta_{\mathrm{C}}$, type \\
\hline 1 & & $166.9, \mathrm{C}$ & & $166.9, \mathrm{C}$ \\
\hline 3 & & 154.6, C & & $154.6, \mathrm{C}$ \\
\hline 4 & $6.28, \mathrm{~s}$ & 106.1, CH & $6.28, \mathrm{~s}$ & 106.1, CH \\
\hline $4 a$ & & 139.0, C & & $139.0, \mathrm{C}$ \\
\hline 5 & $6.33, \mathrm{~d}(2.3)$ & $101.4, \mathrm{CH}$ & $6.33, \mathrm{~d}(2.3)$ & $101.4, \mathrm{CH}$ \\
\hline 6 & & $166.2, \mathrm{C}$ & & $166.2, \mathrm{C}$ \\
\hline 7 & $6.46, \mathrm{~d}(2.3)$ & $100.5, \mathrm{CH}$ & $6.47, \mathrm{~d}(2.3)$ & $100.5, \mathrm{CH}$ \\
\hline 8 & & 163.6, C & & $163.7, \mathrm{C}$ \\
\hline $8 \mathrm{a}$ & & $100.0, \mathrm{C}$ & & $100.0, \mathrm{C}$ \\
\hline 9 & $2.59, \mathrm{~m} ; 2.65, \mathrm{~m}$ & $43.0, \mathrm{CH}_{2}$ & $\begin{array}{l}2.59, \mathrm{dd}(8.0,14.5) ; 2.65, \mathrm{dd} \\
(4.4,14.5)\end{array}$ & $43.0, \mathrm{CH}_{2}$ \\
\hline 10 & $4.27, \mathrm{~m}$ & $65.5, \mathrm{CH}$ & $4.27, \mathrm{~m}$ & $65.5, \mathrm{CH}$ \\
\hline 11 & $1.31, \mathrm{~d}(6.2)$ & $23.3, \mathrm{CH}_{3}$ & $1.33, \mathrm{~d}(6.3)$ & $23.3, \mathrm{CH}_{3}$ \\
\hline 6-OMe & $3.86, \mathrm{~s}$ & $55.7, \mathrm{CH}_{3}$ & $3.86, \mathrm{~s}$ & $55.7, \mathrm{CH}_{3}$ \\
\hline $8-\mathrm{OH}$ & $11.03, \mathrm{~s}$ & & $11.01, \mathrm{~s}$ & \\
\hline
\end{tabular}

NMR spectrum (500 MHz) for ${ }^{1} \mathrm{H}$, NMR spectrum $(125 \mathrm{MHz})$ for ${ }^{13} \mathrm{C}, \mathrm{CDCl}_{3}$, "m" means overlapped or multiple with other signals. Chemical shifts are reported in ppm.

HRMS (ESI, $\mathrm{M}+\mathrm{H}^{+}$) $\mathrm{m} / z$ calculated for $\mathrm{C}_{13} \mathrm{H}_{15} \mathrm{O}_{5}{ }^{+}$251.0914; found 251.0922. 
Table S9. Spectroscopic data of compound 6<smiles>CC(=O)CC(=O)Cc1cc2cc(O)cc(O)c2c(=O)o1</smiles>

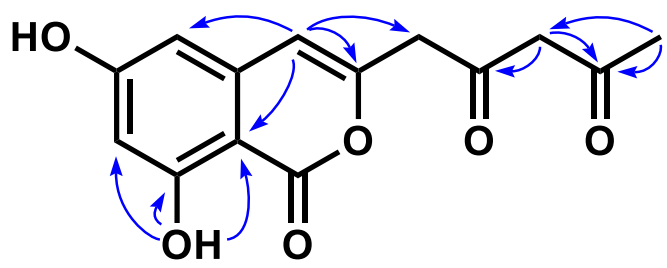

6A

Key HMBC correlations
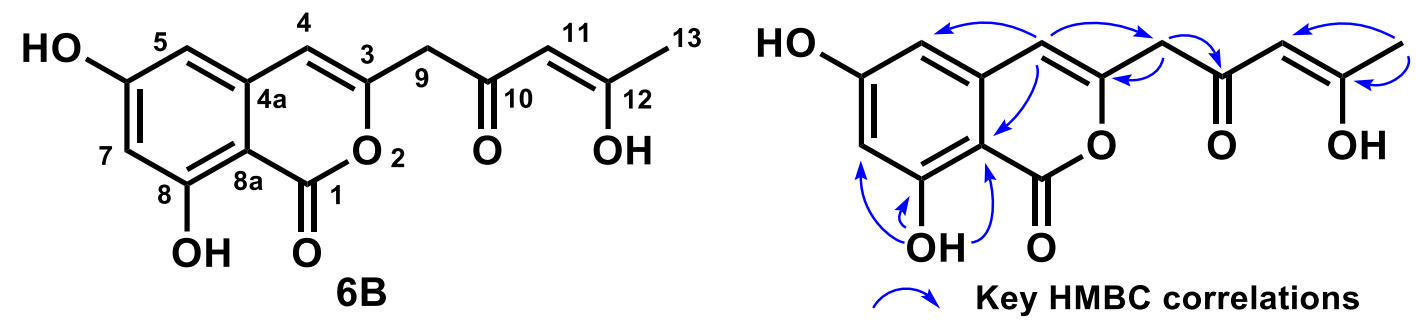

\begin{tabular}{|c|c|c|c|c|}
\hline \multirow[b]{2}{*}{ Position } & \multicolumn{2}{|c|}{ 6A } & \multicolumn{2}{|c|}{$6 B$} \\
\hline & $\delta_{\mathrm{H}}(J$ in $\mathrm{Hz})$ & $\delta_{\mathrm{C}}$, type & $\delta_{\mathrm{H}}(J$ in $\mathrm{Hz})$ & $\delta_{\mathrm{C}}$, type \\
\hline 1 & & 165.2, C & & 165.2, C \\
\hline 3 & & $150.5, \mathrm{C}$ & & 151.1, C \\
\hline 4 & $6.57, \mathrm{~s}$ & 107.0, $\mathrm{CH}$ & $6.63, \mathrm{~s}$ & $107.3, \mathrm{CH}$ \\
\hline $4 a$ & & $139.1, \mathrm{C}$ & & $139.1, \mathrm{C}$ \\
\hline 5 & $6.40, \mathrm{~m}$ & 103.1, CH & $6.40, \mathrm{~m}$ & $103.2, \mathrm{CH}$ \\
\hline 6 & & $165.8, \mathrm{C}$ & & $165.8, \mathrm{C}$ \\
\hline 7 & $6.35, \mathrm{~m}$ & 102.0, $\mathrm{CH}$ & $6.35, \mathrm{~m}$ & $102.0 \mathrm{CH}$ \\
\hline 8 & & 162.7, C & & 162.7, C \\
\hline $8-\mathrm{OH}$ & $10.87, \mathrm{~s}$ & & $10.90, \mathrm{~s}$ & \\
\hline $8 \mathrm{a}$ & & $98.2, \mathrm{C}$ & & $98.2, \mathrm{C}$ \\
\hline 9 & $3.83, \mathrm{~s}$ & 47.0, $\mathrm{CH}_{2}$ & $3.65, \mathrm{~s}$ & $42.4, \mathrm{CH}_{2}$ \\
\hline 10 & & $200.4, \mathrm{C}$ & & $190.9, \mathrm{C}$ \\
\hline 11 & $3.82, \mathrm{~s}$ & $56.6, \mathrm{CH}_{2}$ & $5.79, \mathrm{~s}$ & $100.2, \mathrm{CH}$ \\
\hline 12 & & 203.0, C & & 189.3, C \\
\hline 13 & $2.16, \mathrm{~s}$ & $30.8, \mathrm{CH}_{3}$ & $2.05, \mathrm{~s}$ & $23.8, \mathrm{CH}_{3}$ \\
\hline
\end{tabular}

NMR spectrum $(500 \mathrm{MHz})$ for ${ }^{1} \mathrm{H}$, NMR spectrum $(125 \mathrm{MHz})$ for ${ }^{13} \mathrm{C}, \mathrm{DMSO}-d_{6}$, "m" means overlapped or multiple with other signals. Chemical shifts are reported in ppm.

6 was obtained as white powder. The Structure elucidation indicated 6 was existed as $6 \mathrm{~A}$ and $6 \mathrm{~B}$. (The ratio of $6 \mathrm{~A}$ and 6B was 1:1.5 in DMSO- $d_{6}$ based on NMR peak integration).

HRMS (ESI, $\mathrm{M}+\mathrm{H}^{+}$) m/z calculated for $\mathrm{C}_{14} \mathrm{H}_{13} \mathrm{O}_{6}{ }^{+}$277.0707; found 277.0707. 
Table S10. Spectroscopic data of compound 8-methyldiaporthin (7)

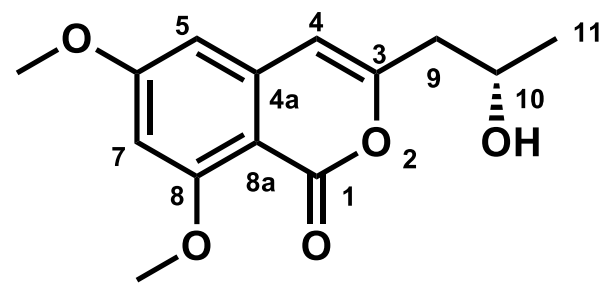

\section{8-methyldiaporthin (7)}

\begin{tabular}{c|c|c}
\hline & $\begin{array}{c}\text { 8-methyldiaporthin (7) in DMSO- } d_{6} \\
\text { Position }\end{array}$ & $\begin{array}{c}\text { Reported 8-methyldiaporthin (7) in DMSO- } d_{6} \\
(6000 \mathrm{MHz})\end{array}$ \\
\hline 1 & $\delta_{\mathrm{C}}, \mathrm{type}$ & $\delta_{\mathrm{C}}$, type \\
3 & $157.9, \mathrm{C}$ & $157.9, \mathrm{C}$ \\
4 & $156.3, \mathrm{C}$ & $156.3, \mathrm{C}$ \\
$4 \mathrm{a}$ & $104.3, \mathrm{CH}$ & $104.2, \mathrm{CH}$ \\
5 & $141.9, \mathrm{C}$ & $141.9, \mathrm{C}$ \\
6 & $100.3, \mathrm{CH}$ & $100.2, \mathrm{CH}$ \\
7 & $165.1, \mathrm{C}$ & $165.1, \mathrm{C}$ \\
8 & $98.3, \mathrm{CH}$ & $98.3, \mathrm{CH}$ \\
$8 \mathrm{a}$ & $162.7, \mathrm{C}$ & $162.7, \mathrm{C}$ \\
9 & $101.9, \mathrm{C}$ & $101.9, \mathrm{C}$ \\
10 & $42.7, \mathrm{CH}_{2}$ & $42.7, \mathrm{CH}_{2}$ \\
11 & $64.0, \mathrm{CH}^{2}$ & $63.9, \mathrm{CH}$ \\
$6-\mathrm{OMe}$ & $23.4, \mathrm{CH}_{3}$ & $23.3, \mathrm{CH}_{3}$ \\
$8-\mathrm{OMe}$ & $55.8, \mathrm{CH}_{3}$ & $55.7, \mathrm{CH}_{3}$ \\
\hline
\end{tabular}

NMR spectrum (500 MHz) for ${ }^{1} \mathrm{H}$, NMR spectrum (125 MHz) for ${ }^{13} \mathrm{C}$, DMSO- $d_{6}$, "m" means overlapped or multiple with other signals. Chemical shifts are reported in ppm.

HRMS (ESI, $\mathrm{M}+\mathrm{H}^{+}$) $m / z$ calculated for $\mathrm{C}_{14} \mathrm{H}_{17} \mathrm{O}_{5}{ }^{+} 265.1071$; found 265.1074. 
Table S11. Spectroscopic data of compound 8
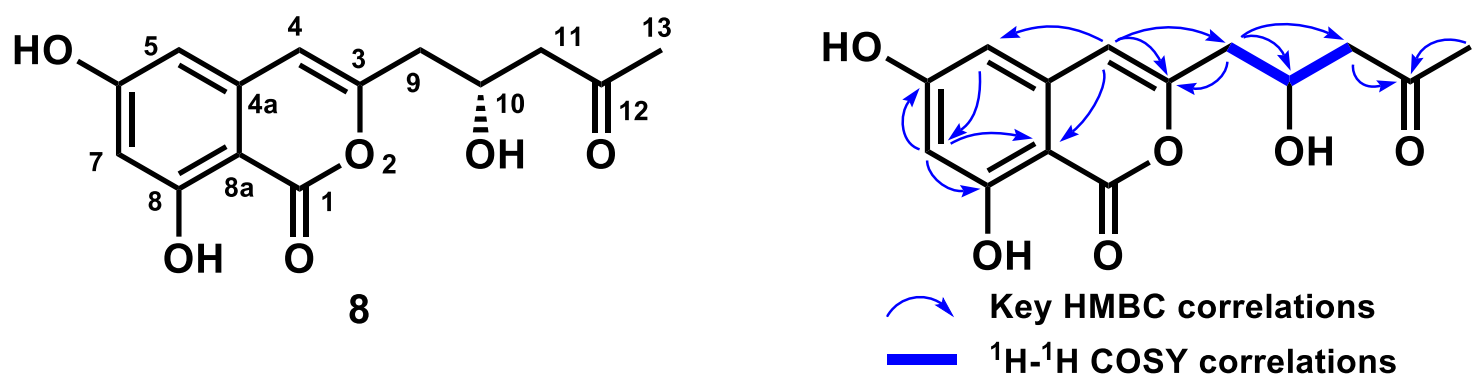

${ }^{1} \mathrm{H}$ NMR spectrum (500 MHz), ${ }^{13} \mathrm{C}$ NMR spectrum (125 MHz), $\mathrm{CD}_{3} \mathrm{OD}$

\begin{tabular}{|c|c|c|c|}
\hline \multirow[b]{2}{*}{ Position } & \multicolumn{2}{|c|}{$\begin{array}{c}\text { Citreoisocoumarin }(\mathbf{8}) \text { in } \mathrm{CD}_{3} \mathrm{OD} \\
(500 \mathrm{MHz})\end{array}$} & \multirow{2}{*}{ 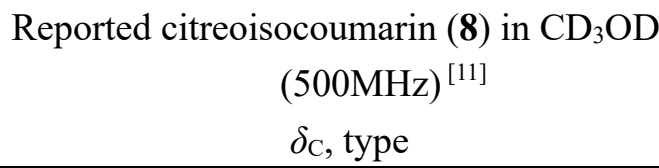 } \\
\hline & $\delta_{\mathrm{H}}(J$ in $\mathrm{Hz})$ & $\delta_{\mathrm{C}}$, type & \\
\hline 1 & & 167.6, C & 167.8, C \\
\hline 3 & & 155.6, C & 155.6, C \\
\hline 4 & $6.39, \mathrm{~s}$ & 107.4, CH & $107.4, \mathrm{CH}$ \\
\hline $4 a$ & & 141.1, C & 141.1, C \\
\hline 5 & $6.32, \mathrm{~s}$ & $103.8, \mathrm{CH}$ & $103.8, \mathrm{CH}$ \\
\hline 6 & & 167.2, C & 167.3, C \\
\hline 7 & $6.32, \mathrm{~s}$ & $102.7, \mathrm{CH}$ & 102.7, $\mathrm{CH}$ \\
\hline 8 & & $164.8, \mathrm{C}$ & 164.8, C \\
\hline $8 a$ & & $99.8, \mathrm{C}$ & 99.9, C \\
\hline 9 & $2.63, \mathrm{~m}$ & $41.9, \mathrm{CH}_{2}$ & $41.9, \mathrm{CH}_{2}$ \\
\hline 10 & $4.45, \mathrm{~m}$ & $66.4, \mathrm{CH}$ & $66.4, \mathrm{CH}$ \\
\hline 11 & $2.69, \mathrm{~m}$ & $51.1, \mathrm{CH}_{2}$ & $51.1, \mathrm{CH}_{2}$ \\
\hline 12 & & 209.7, C & 209.8, C \\
\hline 13 & $2.18, \mathrm{~s}$ & $30.7, \mathrm{CH}_{3}$ & $30.7, \mathrm{CH}_{3}$ \\
\hline
\end{tabular}

NMR spectrum (500 MHz) for ${ }^{1} \mathrm{H}, \mathrm{NMR}$ spectrum $(125 \mathrm{MHz})$ for ${ }^{13} \mathrm{C}, \mathrm{CD}_{3} \mathrm{OD}$, "m" means overlapped or multiple with other signals. Chemical shifts are reported in ppm.

HRMS (ESI, $\mathrm{M}+\mathrm{H}^{+}$) $m / z$ calculated for $\mathrm{C}_{14} \mathrm{H}_{15} \mathrm{O}_{6}{ }^{+} 279.0863$; found 279.0862.

$$
[\alpha]_{\mathrm{D}}^{24.1}+34^{\circ}(c 0.1, \mathrm{MeOH}) \text {. }
$$


Table S12. Spectroscopic data of compound 9
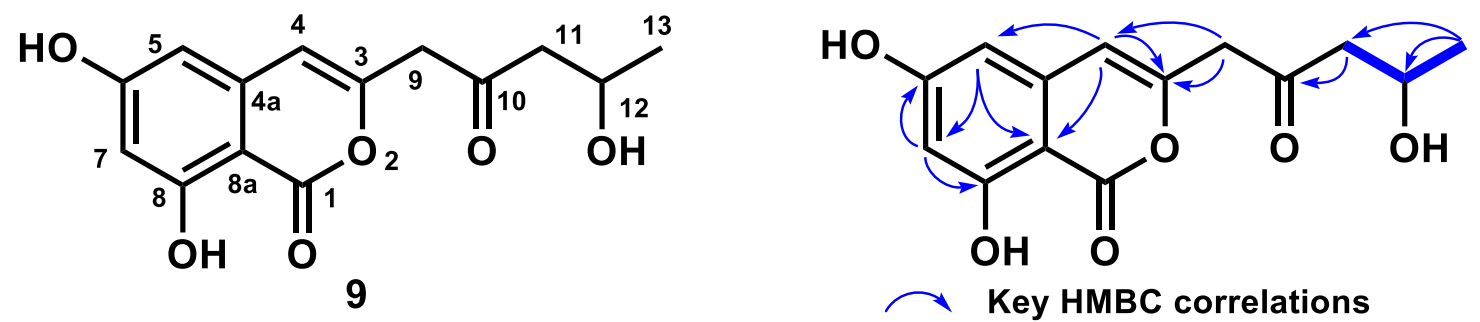

${ }^{1} \mathrm{H}$ NMR spectrum (500 MHz), ${ }^{13} \mathrm{C}$ NMR spectrum (125 MHz), $\mathrm{CD}_{3} \mathrm{OD}$

\begin{tabular}{c|ll}
\hline & \multicolumn{2}{|c}{9} \\
Position & $\delta_{\mathrm{H}}(J$ in $\mathrm{Hz})$ & $\delta_{\mathrm{C}}$, type \\
\hline 1 & & $167.5, \mathrm{C}$ \\
3 & & $152.0, \mathrm{C}$ \\
4 & $6.44, \mathrm{~s}$ & $108.5, \mathrm{CH}$ \\
$4 \mathrm{a}$ & & $140.8, \mathrm{C}$ \\
5 & $6.34, \mathrm{~m}$ & $104.1, \mathrm{CH}$ \\
6 & & $167.4, \mathrm{C}$ \\
7 & $6.34, \mathrm{~m}$ & $103.0, \mathrm{CH}$ \\
8 & & $164.9, \mathrm{C}$ \\
$8 \mathrm{a}$ & & $99.7, \mathrm{C}$ \\
9 & $3.71, \mathrm{~m}$ & $47.3, \mathrm{CH}_{2}$ \\
10 & & $205.9, \mathrm{C}$ \\
11 & $2.69, \mathrm{~m}$ & $52.2, \mathrm{CH}_{2}$ \\
12 & $4.24, \mathrm{~m}$ & $64.9, \mathrm{CH}$ \\
13 & $1.21, \mathrm{~d}(6.3)$ & $23.5, \mathrm{CH}_{3}$ \\
\hline
\end{tabular}

NMR spectrum (500 MHz) for ${ }^{1} \mathrm{H}$, NMR spectrum (125 MHz) for ${ }^{13} \mathrm{C}, \mathrm{CD}_{3} \mathrm{OD}$, “m” means overlapped or multiple with other signals. Chemical shifts are reported in ppm.

HRMS (ESI, $\mathrm{M}+\mathrm{H}^{+}$) $\mathrm{m} / z$ calculated for $\mathrm{C}_{14} \mathrm{H}_{15} \mathrm{O}_{6}{ }^{+} 279.0863$; found 279.0864 .

$$
[\alpha]_{\mathrm{D}}^{24.1}-8.7^{\circ}(c 0.1, \mathrm{MeOH}) \text {. }
$$


Table S13. Spectroscopic data of compound $\mathbf{1 0}$
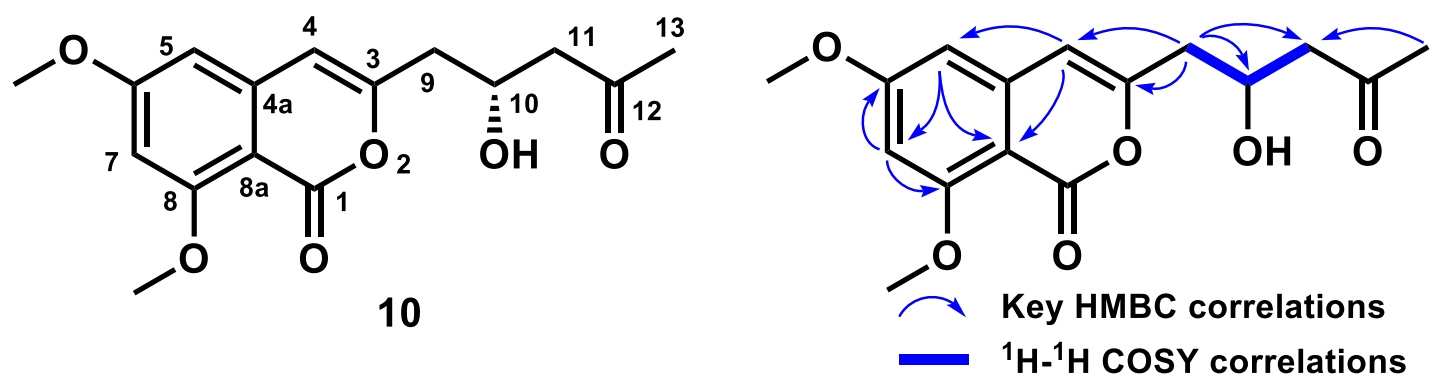

\begin{tabular}{c|ll}
\hline & \multicolumn{2}{|c}{$\mathbf{1 0}$} \\
Position & $\delta_{\mathrm{H}}(J$ in $\mathrm{Hz})$ & $\delta_{\mathrm{C}}$, type \\
\hline 1 & & $161.9, \mathrm{C}$ \\
3 & & $156.6, \mathrm{C}$ \\
4 & $6.40, \mathrm{~s}$ & $106.9, \mathrm{CH}$ \\
$4 \mathrm{a}$ & & $143.8, \mathrm{C}$ \\
5 & $6.54, \mathrm{~d}(2.3)$ & $101.4, \mathrm{CH}$ \\
6 & & $164.6, \mathrm{C}$ \\
7 & $6.57, \mathrm{~s}$ & $99.5, \mathrm{CH}$ \\
8 & & $167.6, \mathrm{C}$ \\
$8 \mathrm{a}$ & & $103.4, \mathrm{C}$ \\
9 & $2.64, \mathrm{~m}$ & $42.0, \mathrm{CH}_{2}$ \\
10 & $4.46, \mathrm{~m}$ & $66.4, \mathrm{CH}^{2}$ \\
11 & $2.70, \mathrm{~m}$ & $51.1, \mathrm{CH}_{2}$ \\
12 & & $209.8, \mathrm{C}$ \\
13 & $2.19, \mathrm{~m}$ & $30.6, \mathrm{CH}_{3}$ \\
$6-\mathrm{OMe}$ & $3.91, \mathrm{~m}$ & $56.3, \mathrm{CH}_{3}$ \\
$8-\mathrm{OMe}$ & $3.91, \mathrm{~m}$ & $56.6, \mathrm{CH}_{3}$ \\
\hline
\end{tabular}

NMR spectrum (500 MHz) for ${ }^{1} \mathrm{H}$, NMR spectrum (125 MHz) for ${ }^{13} \mathrm{C}, \mathrm{CD}_{3} \mathrm{OD}$, “m” means overlapped or multiple with other signals. Chemical shifts are reported in ppm.

HRMS (ESI, $\mathrm{M}+\mathrm{H}^{+}$) $\mathrm{m} / z$ calculated for $\mathrm{C}_{16} \mathrm{H}_{19} \mathrm{O}_{6}{ }^{+}$307.1176; found 307.1171.

$$
[\alpha]_{\mathrm{D}}^{24.1}+18.7^{\circ}(c 0.1, \mathrm{MeOH}) .
$$


Table S14. Spectroscopic data of compound 12
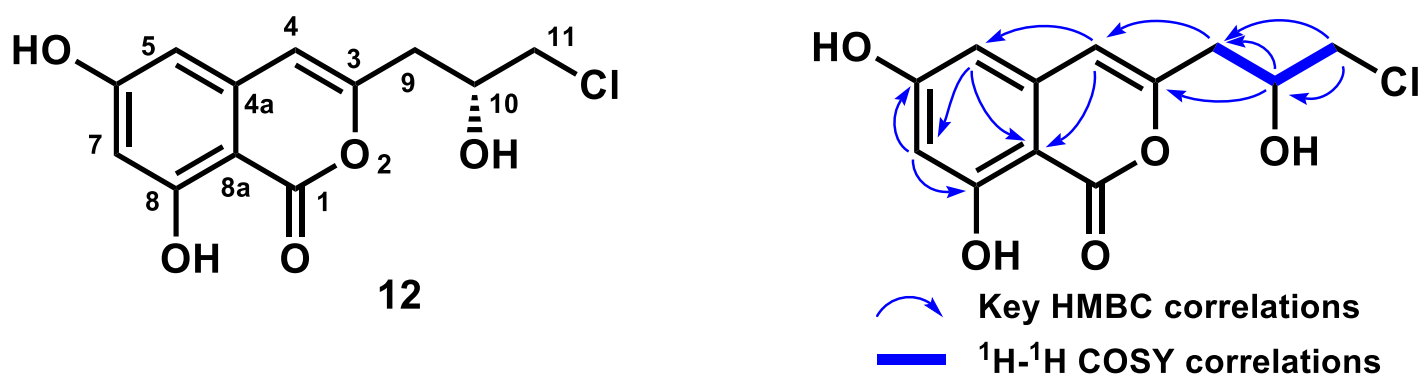

${ }^{1} \mathrm{H}$ NMR spectrum $(500 \mathrm{MHz}),{ }^{13} \mathrm{C}$ NMR spectrum (125 MHz), $\mathrm{CD}_{3} \mathrm{OD}$

\begin{tabular}{c|ll}
\hline & \multicolumn{2}{|c}{12} \\
Position & $\delta_{\mathrm{H}}(J$ in $\mathrm{Hz})$ & $\delta_{\mathrm{C}}$, type \\
\hline 1 & & $167.6, \mathrm{C}$ \\
3 & & $155.2, \mathrm{C}$ \\
4 & $6.38, \mathrm{~s}$ & $107.4, \mathrm{CH}$ \\
$4 \mathrm{a}$ & & $141.0, \mathrm{C}$ \\
5 & $6.29, \mathrm{~s}$ & $103.8, \mathrm{C}$ \\
6 & & $167.3, \mathrm{C}$ \\
7 & $6.30, \mathrm{~s}$ & $102.7, \mathrm{CH}$ \\
8 & & $164.8, \mathrm{C}$ \\
$8 \mathrm{a}$ & & $99.8, \mathrm{C}$ \\
9 & $2.64, \mathrm{dd}(8.5,14.6) ; 2.83$, & $39.2, \mathrm{CH}_{2}$ \\
& $\mathrm{dd}(4.5,14.7)$ & \\
10 & $4.19, \mathrm{~m}$ & $69.5, \mathrm{CH}^{2}$ \\
11 & $3.62, \mathrm{~m}$ & $49.5, \mathrm{CH}_{2}$ \\
\hline
\end{tabular}

NMR spectrum (500 MHz) for ${ }^{1} \mathrm{H}$, NMR spectrum (125 MHz) for ${ }^{13} \mathrm{C}, \mathrm{CD}_{3} \mathrm{OD}$, "m" means overlapped or multiple with other signals. Chemical shifts are reported in ppm.

HRMS (ESI, $\left.\mathrm{M}+\mathrm{H}^{+}\right) \mathrm{m} / z$ calculated for $\mathrm{C}_{12} \mathrm{H}_{12} \mathrm{ClO}_{5}{ }^{+} 271.0368$; found 271.0366.

$$
[\alpha]_{\mathrm{D}}^{24.1}+18^{\circ}(c 0.1, \mathrm{MeOH}) .
$$


Table S15. Spectroscopic data of compound $\mathbf{1 3}$

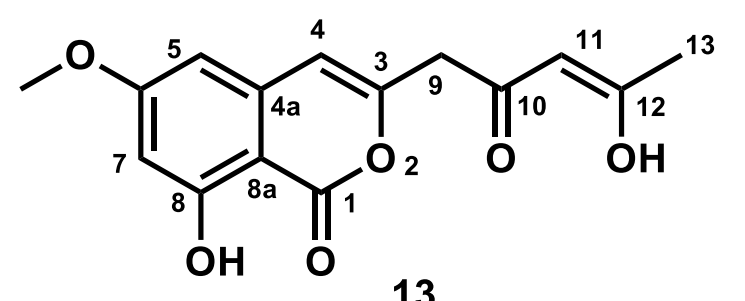

13

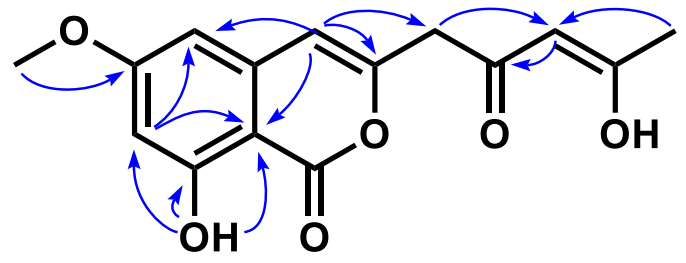

Key HMBC correlations

\begin{tabular}{c|ll}
\hline & & 13 \\
Position & $\delta_{\mathrm{H}}(J$ in $\mathrm{Hz})$ & $\delta_{\mathrm{C}}$, type \\
\hline 1 & & $165.9, \mathrm{C}$ \\
3 & & $150.9, \mathrm{C}$ \\
4 & $6.35, \mathrm{~m}$ & $107.2, \mathrm{CH}$ \\
$4 \mathrm{a}$ & & $138.7, \mathrm{C}$ \\
5 & $6.35, \mathrm{~m}$ & $102.0, \mathrm{CH}$ \\
6 & & $167.0, \mathrm{C}$ \\
7 & $6.47, \mathrm{~s}$ & $101.0, \mathrm{CH}$ \\
8 & & $163.8, \mathrm{C}$ \\
$8 \mathrm{a}$ & & $100.0, \mathrm{C}$ \\
9 & $3.45, \mathrm{~s}$ & $43.1, \mathrm{CH}_{2}$ \\
10 & & $189.0, \mathrm{C}$ \\
11 & $5.61, \mathrm{~s}$ & $100.3, \mathrm{CH}$ \\
12 & & $190.4, \mathrm{C}$ \\
13 & $2.06, \mathrm{~s}$ & $24.5, \mathrm{CH}_{3}$ \\
$6-\mathrm{OMe}$ & $3.85, \mathrm{~s}$ & $55.8, \mathrm{CH}_{3}$ \\
\hline
\end{tabular}

NMR spectrum (500 MHz) for ${ }^{1} \mathrm{H}$, NMR spectrum $(125 \mathrm{MHz})$ for ${ }^{13} \mathrm{C}, \mathrm{CDCl}_{3}$, "m" means overlapped or multiple with other signals. Chemical shifts are reported in ppm.

HRMS (ESI, $\mathrm{M}+\mathrm{H}^{+}$) m/z calculated for $\mathrm{C}_{15} \mathrm{H}_{15} \mathrm{O}_{6}{ }^{+}$291.0863; found 291.0863. 
Table S16. Spectroscopic data of compound 14

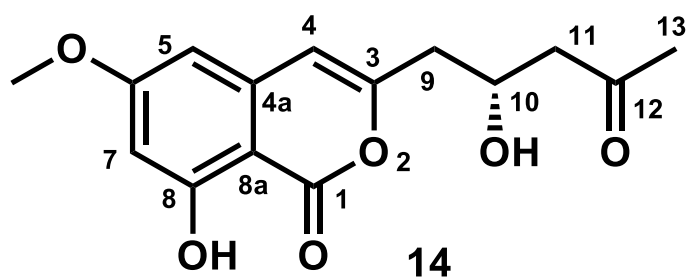

${ }^{1} \mathrm{H}$ NMR spectrum $(500 \mathrm{MHz}),{ }^{13} \mathrm{C}$ NMR spectrum (125 MHz), $\mathrm{CDCl}_{3}$

\begin{tabular}{|c|c|c|c|c|}
\hline \multirow[b]{2}{*}{ Position } & \multicolumn{2}{|c|}{$\begin{array}{l}\text { 6-O-methylcitreoisocoumarin (14) in } \\
\qquad \mathrm{CDCl}_{3}(500 \mathrm{MHz})\end{array}$} & \multicolumn{2}{|c|}{$\begin{array}{l}\text { Reported 6-O-methylcitreoisocoumarin (14) in } \\
\qquad \mathrm{CDCl}_{3} \\
(500 \mathrm{MHz})^{12}\end{array}$} \\
\hline & $\delta_{\mathrm{H}}(J$ in $\mathrm{Hz})$ & $\delta_{\mathrm{C}}$, type & $\delta_{\mathrm{H}}(J$ in $\mathrm{Hz})$ & $\delta_{\mathrm{C}}$, type \\
\hline 1 & & 166.2, C & & 166.3, C \\
\hline 3 & & 154.0, C & & $153.9, \mathrm{C}$ \\
\hline 4 & $6.30, \mathrm{~m}$ & $106.5, \mathrm{CH}$ & $6.32, \mathrm{~s}$ & $106.5, \mathrm{CH}$ \\
\hline $4 a$ & & 139.1, C & & 139.1, C \\
\hline 5 & $6.30, \mathrm{~m}$ & $101.5, \mathrm{CH}$ & $6.33, \mathrm{~d}(2.2)$ & 101.6, CH \\
\hline 6 & & $166.9, \mathrm{C}$ & & 167.0, C \\
\hline 7 & $6.42, \mathrm{~d}(2.3)$ & $100.7, \mathrm{CH}$ & $6.46, \mathrm{~d}(2.2)$ & $100.7, \mathrm{CH}$ \\
\hline 8 & & $163.6, \mathrm{C}$ & & 163.7, C \\
\hline $8 \mathrm{a}$ & & $100.0, \mathrm{C}$ & & 100.1, C \\
\hline 9 & $2.66, \mathrm{~m}$ & $40.2, \mathrm{CH}_{2}$ & $2.63, \mathrm{dd}(5.3,14.8)$ & $40.2, \mathrm{CH}_{2}$ \\
\hline & & & $2.70, \mathrm{dd}(7.6,13.1)$ & \\
\hline 10 & $4.46, \mathrm{~m}$ & $65.3, \mathrm{CH}$ & $4.47, \mathrm{~m}$ & $65.4, \mathrm{CH}$ \\
\hline 11 & $2.66, \mathrm{~m}$ & $49.1, \mathrm{CH}_{2}$ & $2.67, \mathrm{dd}(7.5,17.8)$ & $49.1, \mathrm{CH}_{2}$ \\
\hline & & & $2.76, \mathrm{dd}(3.0,17.9)$ & \\
\hline 12 & & 209.2, C & & 209.3, C \\
\hline 13 & $2.18, \mathrm{~s}$ & $30.9, \mathrm{CH}_{3}$ & $2.21, \mathrm{~s}$ & $30.9, \mathrm{CH}_{3}$ \\
\hline 6-OMe & $3.83, \mathrm{~s}$ & $55.8, \mathrm{CH}_{3}$ & $3.86, \mathrm{~s}$ & $55.8, \mathrm{CH}_{3}$ \\
\hline $8-\mathrm{OH}$ & $10.97, \mathrm{~s}$ & & $11.03, \mathrm{~s}$ & \\
\hline
\end{tabular}

NMR spectrum (500 MHz) for ${ }^{1} \mathrm{H}$, NMR spectrum (125 MHz) for ${ }^{13} \mathrm{C}, \mathrm{CDCl}_{3}$, "m" means overlapped or multiple with other signals. Chemical shifts are reported in ppm.

HRMS (ESI, $\mathrm{M}+\mathrm{H}^{+}$) $m / z$ calculated for $\mathrm{C}_{15} \mathrm{H}_{17} \mathrm{O}_{6}{ }^{+}$293.1020; found 293.1025 .

$$
[\alpha]_{\mathrm{D}}^{24.1}+16.0^{\circ}(c 0.1, \mathrm{MeOH}) .
$$


Table S17. Spectroscopic data of compound 15<smiles>CC(=O)C(Cl)C(=O)Cc1cc2cc(O)cc(O)c2c(=O)o1</smiles>

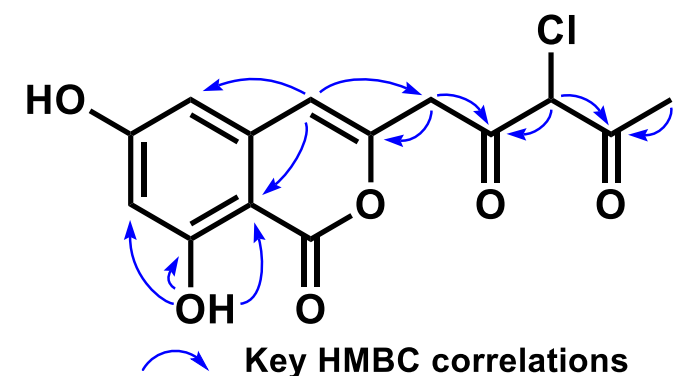<smiles>CC(O)=C(Cl)C(=O)Cc1cc2cc(O)cc(O)c2c(=O)o1</smiles>

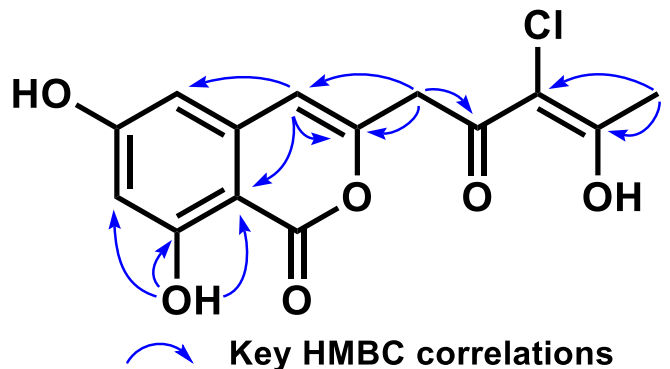

\begin{tabular}{|c|c|c|c|c|}
\hline \multirow[b]{2}{*}{ Position } & \multicolumn{2}{|c|}{$15 \mathrm{~A}$} & \multicolumn{2}{|c|}{ 15B } \\
\hline & $\delta_{\mathrm{H}}(J$ in $\mathrm{Hz})$ & $\delta_{\mathrm{C}}$, type & $\delta_{\mathrm{H}}(J$ in $\mathrm{Hz})$ & $\delta_{\mathrm{C}}$, type \\
\hline 1 & & 166.1, C & & 166.1, C \\
\hline 3 & & $150.4, \mathrm{C}$ & & $151.5, \mathrm{C}$ \\
\hline 4 & $6.47, \mathrm{~s}$ & 108.7, CH & $6.47, \mathrm{~s}$ & $108.0, \mathrm{CH}$ \\
\hline $4 a$ & & $140.1, \mathrm{C}$ & & $140.3, \mathrm{C}$ \\
\hline 5 & $6.40, \mathrm{~m}$ & $103.8, \mathrm{CH}$ & $6.40, \mathrm{~m}$ & 104.0, CH \\
\hline 6 & & $166.7, \mathrm{C}$ & & $166.8, \mathrm{C}$ \\
\hline 7 & $6.40, \mathrm{~m}$ & $102.9, \mathrm{CH}$ & $6.40, \mathrm{~m}$ & $103.0 \mathrm{CH}$ \\
\hline 8 & & $164.5, \mathrm{C}$ & & $164.5, \mathrm{C}$ \\
\hline $8-\mathrm{OH}$ & 10.96, s & & 10.97, s & \\
\hline $8 \mathrm{a}$ & & $100.1, \mathrm{C}$ & & $100.2, \mathrm{C}$ \\
\hline 9 & $3.91, \mathrm{~m}$ & $45.1, \mathrm{CH}_{2}$ & $3.88, \mathrm{~m}$ & $41.5, \mathrm{CH}_{2}$ \\
\hline 10 & & 196.7, C & & 188.3, C \\
\hline 11 & $5.30, \mathrm{~s}$ & $67.8, \mathrm{CH}$ & & 108.8, C \\
\hline 12 & & 199.2, C & & $188.5, \mathrm{C}$ \\
\hline 13 & $2.34, \mathrm{~s}$ & $28.0, \mathrm{CH}_{3}$ & $2.29, \mathrm{~s}$ & $23.4, \mathrm{CH}_{3}$ \\
\hline
\end{tabular}

NMR spectrum (500 MHz) for ${ }^{1} \mathrm{H}$, NMR spectrum $(125 \mathrm{MHz})$ for ${ }^{13} \mathrm{C}$, acetonitrile- $d_{3}$, "m" means overlapped or multiple with other signals. Chemical shifts are reported in ppm.

15 was obtained as brown-yellow oil. The Structure elucidation indicated 15 was existed as 15A and 15B. (The ratio of 15A and 15B was 1:1 in acetonitrile- $d_{3}$ based on NMR peak integration).

HRMS (ESI, $\mathrm{M}+\mathrm{H}^{+}$) $\mathrm{m} / z$ calculated for $\mathrm{C}_{14} \mathrm{H}_{12} \mathrm{ClO}_{6}{ }^{+}$311.0317; found 311.0311. 
Table S18. Spectroscopic data of compound 16

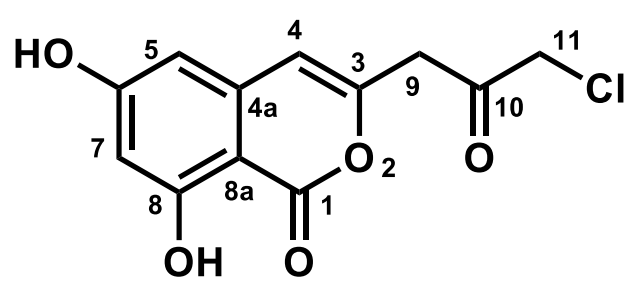

16

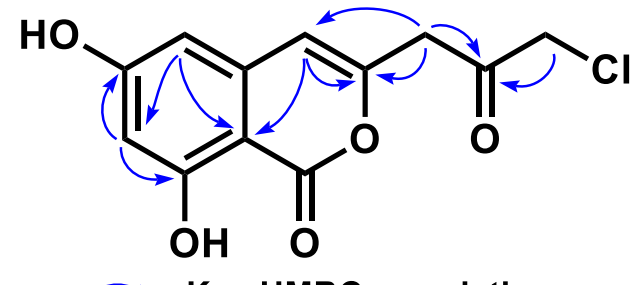

$\frown$ Key HMBC correlations

${ }^{1} \mathrm{H}$ NMR spectrum (500 MHz), ${ }^{13} \mathrm{C}$ NMR spectrum (125 MHz), Acetonitrile- $d_{3}$

\begin{tabular}{c|ll}
\hline & \multicolumn{2}{|c}{$\mathbf{1 6}$} \\
Position & $\delta_{\mathrm{H}}(J$ in Hz $)$ & $\delta_{\mathrm{C}}$, type \\
\hline 1 & & $166.8, \mathrm{C}$ \\
3 & & $150.9, \mathrm{C}$ \\
4 & $6.45, \mathrm{~s}$ & $108.3, \mathrm{CH}$ \\
$4 \mathrm{a}$ & & $140.2, \mathrm{C}$ \\
5 & $6.40, \mathrm{~m}$ & $103.9, \mathrm{C}$ \\
6 & & $166.1, \mathrm{C}$ \\
7 & $6.40, \mathrm{~m}$ & $108.3, \mathrm{CH}$ \\
8 & & $164.4, \mathrm{C}$ \\
$8 \mathrm{a}$ & & $100.1, \mathrm{C}$ \\
9 & $3.77, \mathrm{~s}$ & $44.8, \mathrm{CH}_{2}$ \\
10 & & $197.8, \mathrm{C}$ \\
11 & $4.43, \mathrm{~m}$ & $49.7, \mathrm{CH}_{2}$ \\
\hline
\end{tabular}

NMR spectrum (500 MHz) for ${ }^{1} \mathrm{H}$, NMR spectrum (125 MHz) for ${ }^{13} \mathrm{C}$, acetonitrile- $d_{3}$, “m” means overlapped or multiple with other signals. Chemical shifts are reported in ppm.

HRMS (ESI, $\mathrm{M}+\mathrm{H}^{+}$) $m / z$ calculated for $\mathrm{C}_{12} \mathrm{H}_{10} \mathrm{ClO}_{5}{ }^{+}$269.0211; found 269.0210. 
Table S19. Spectroscopic data of compound 19
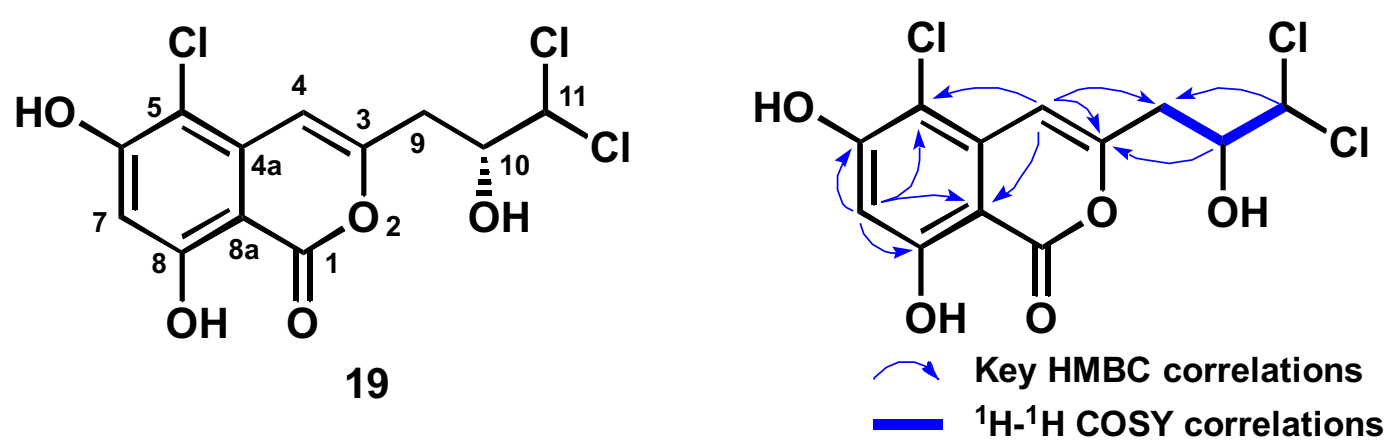

${ }^{1} \mathrm{H}$ NMR spectrum $(500 \mathrm{MHz}),{ }^{13} \mathrm{C}$ NMR spectrum (125 MHz), $\mathrm{CD}_{3} \mathrm{OD}$

\begin{tabular}{c|ll}
\hline & \multicolumn{2}{|c}{19} \\
Position & $\delta_{\mathrm{H}}(J$ in $\mathrm{Hz})$ & $\delta_{\mathrm{C}}$, type \\
\hline 1 & & $166.9, \mathrm{C}$ \\
3 & & $156.0, \mathrm{C}$ \\
4 & $6.78, \mathrm{~s}$ & $104.1, \mathrm{CH}$ \\
$4 \mathrm{a}$ & & $137.3, \mathrm{C}$ \\
5 & & $107.5, \mathrm{C}$ \\
6 & & $163.0, \mathrm{C}$ \\
7 & $6.44, \mathrm{~s}$ & $103.2, \mathrm{CH}$ \\
8 & & $163.0, \mathrm{C}$ \\
$8 \mathrm{a}$ & & $100.1, \mathrm{C}$ \\
9 & $2.80, \mathrm{dd}(9.6,14.8)$ & $37.6, \mathrm{CH}$ \\
& $3.03, \mathrm{dd}(3.3,14.8)$ & \\
10 & $4.31, \mathrm{dt}(3.3,9.6)$ & $74.3, \mathrm{CH}$ \\
11 & $6.04, \mathrm{~d}(3.5)$ & $77.1, \mathrm{CH}$ \\
\hline
\end{tabular}

NMR spectrum (500 MHz) for ${ }^{1} \mathrm{H}, \mathrm{NMR}$ spectrum (125 MHz) for ${ }^{13} \mathrm{C}, \mathrm{CD}_{3} \mathrm{OD}$, "m" means overlapped or multiple with other signals. Chemical shifts are reported in ppm.

HRMS (ESI, $\mathrm{M}+\mathrm{H}^{+}$) $\mathrm{m} / z$ calculated for $\mathrm{C}_{12} \mathrm{H}_{10} \mathrm{Cl}_{3} \mathrm{O}_{5}{ }^{+}$338.9588; found 338.9596 .

$$
[\alpha]_{\mathrm{D}}^{24.1}+16.7^{\circ}(c 0.1, \mathrm{MeOH})
$$


Table S20. Spectroscopic data of compound 20
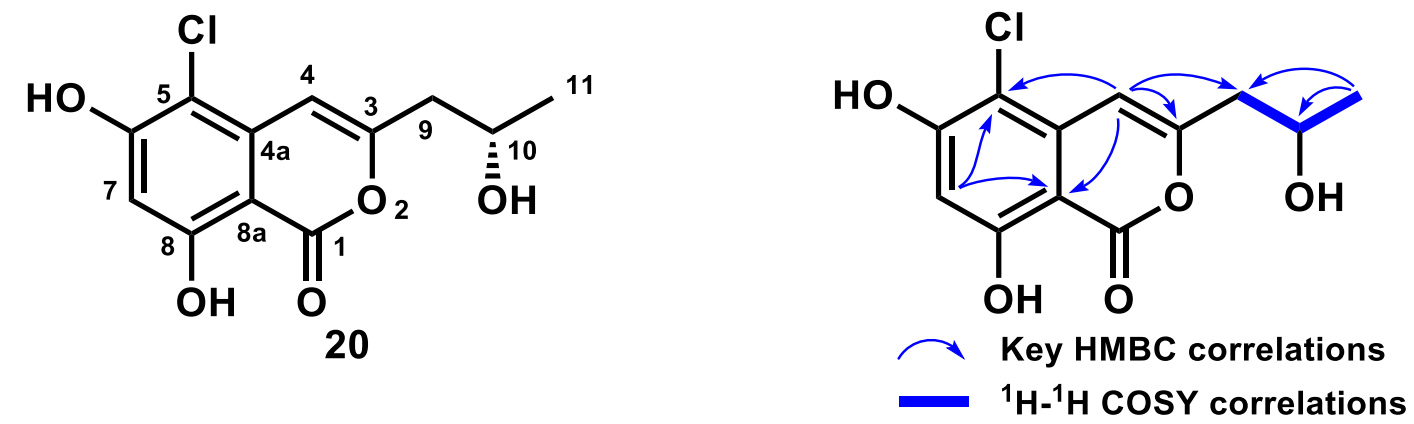

${ }^{1} \mathrm{H}$ NMR spectrum (500 MHz), ${ }^{13} \mathrm{C}$ NMR spectrum (125 MHz), $\mathrm{CD}_{3} \mathrm{OD}$

\begin{tabular}{c|ll}
\hline & \multicolumn{2}{|c}{$\mathbf{2 0}$} \\
Position & $\delta_{\mathrm{H}}(J$ in $\mathrm{Hz})$ & $\delta_{\mathrm{C}}$, type \\
\hline 1 & & $163.0, \mathrm{C}$ \\
3 & & $157.5, \mathrm{C}$ \\
4 & $6.68, \mathrm{~s}$ & $103.4, \mathrm{CH}$ \\
$4 \mathrm{a}$ & & $137.5, \mathrm{C}$ \\
5 & & $107.3, \mathrm{C}$ \\
6 & & $167.2, \mathrm{C}$ \\
7 & $6.40, \mathrm{~s}$ & $103.0, \mathrm{CH}$ \\
8 & & $163.0, \mathrm{C}$ \\
$8 \mathrm{a}$ & & $100.1, \mathrm{C}$ \\
9 & $2.63, \mathrm{~m}$ & $44.1, \mathrm{CH}_{2}$ \\
10 & $4.15, \mathrm{~m}$ & $66.2, \mathrm{CH}$ \\
11 & $1.26, \mathrm{~d}(6.4)$ & $23.4, \mathrm{CH}_{3}$ \\
\hline
\end{tabular}

NMR spectrum (500 MHz) for ${ }^{1} \mathrm{H}$, NMR spectrum (125 MHz) for ${ }^{13} \mathrm{C}, \mathrm{CD}_{3} \mathrm{OD}$, "m" means overlapped or multiple with other signals. Chemical shifts are reported in ppm.

HRMS (ESI, $\mathrm{M}+\mathrm{H}^{+}$) $\mathrm{m} / z$ calculated for $\mathrm{C}_{12} \mathrm{H}_{12} \mathrm{ClO}_{5}{ }^{+} 271.0368$; found 271.0368

$$
[\alpha]_{\mathrm{D}}^{24.1}+20.0^{\circ}(c 0.1, \mathrm{MeOH}) \text {. }
$$


Table S21. Spectroscopic data of compound 21

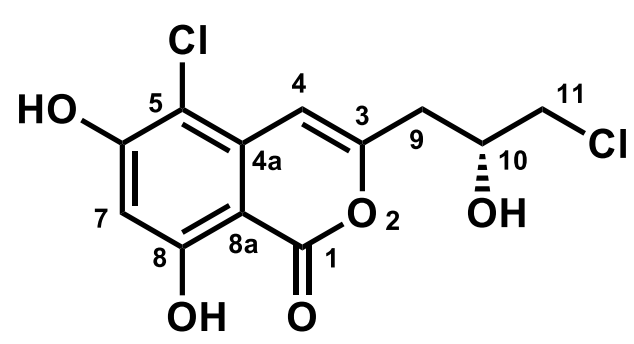

21

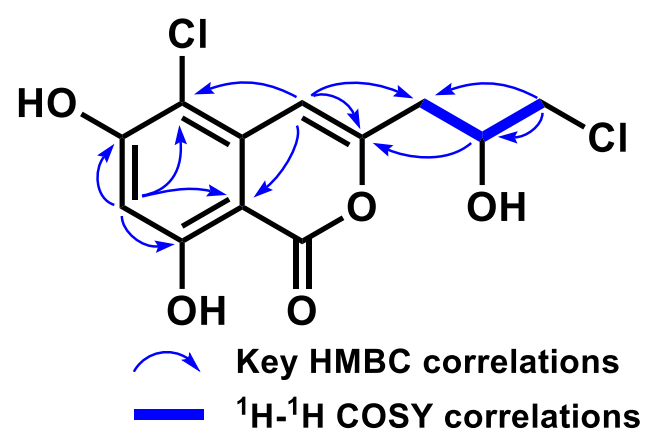

${ }^{1} \mathrm{H}$ NMR spectrum $(500 \mathrm{MHz}),{ }^{13} \mathrm{C}$ NMR spectrum (125 MHz), $\mathrm{CD}_{3} \mathrm{OD}$

\begin{tabular}{c|ll}
\hline & \multicolumn{2}{|c}{21} \\
Position & $\delta_{\mathrm{H}}(J$ in $\mathrm{Hz})$ & $\delta_{\mathrm{C}}$, type \\
\hline 1 & & $163.2, \mathrm{C}$ \\
3 & & $156.6, \mathrm{C}$ \\
4 & $6.79, \mathrm{~s}$ & $103.9, \mathrm{CH}$ \\
$4 \mathrm{a}$ & & $137.4, \mathrm{C}$ \\
5 & & $107.6, \mathrm{C}$ \\
6 & & $167.1, \mathrm{C}$ \\
7 & $6.45, \mathrm{~m}$ & $103.3, \mathrm{CH}$ \\
8 & & $163.6, \mathrm{C}$ \\
$8 \mathrm{a}$ & & $99.9, \mathrm{C}$ \\
9 & $2.71, \mathrm{dd}(8.7,14.6)$ & $39.6, \mathrm{CH}$ \\
& $2.90, \mathrm{dd},(4.2,14.6)$ & \\
10 & $4.21, \mathrm{dq}(4.8,9.4)$ & $69.8, \mathrm{CH}$ \\
11 & $3.65, \mathrm{~m}$ & $49.6, \mathrm{CH}_{2}$ \\
\hline
\end{tabular}

NMR spectrum (500 MHz) for ${ }^{1} \mathrm{H}$, NMR spectrum $(125 \mathrm{MHz})$ for ${ }^{13} \mathrm{C}, \mathrm{CD}_{3} \mathrm{OD}$, “m” means overlapped or multiple with other signals. Chemical shifts are reported in ppm.

HRMS (ESI, $\mathrm{M}+\mathrm{H}^{+}$) $m / z$ calculated for $\mathrm{C}_{12} \mathrm{H}_{11} \mathrm{Cl}_{2} \mathrm{O}_{5}{ }^{+}$304.9978; found 304.9973 .

$$
[\alpha]_{\mathrm{D}}^{24.1}+17.3^{\circ}(c 0.1, \mathrm{MeOH})
$$


Table S22. Spectroscopic data of compound 22
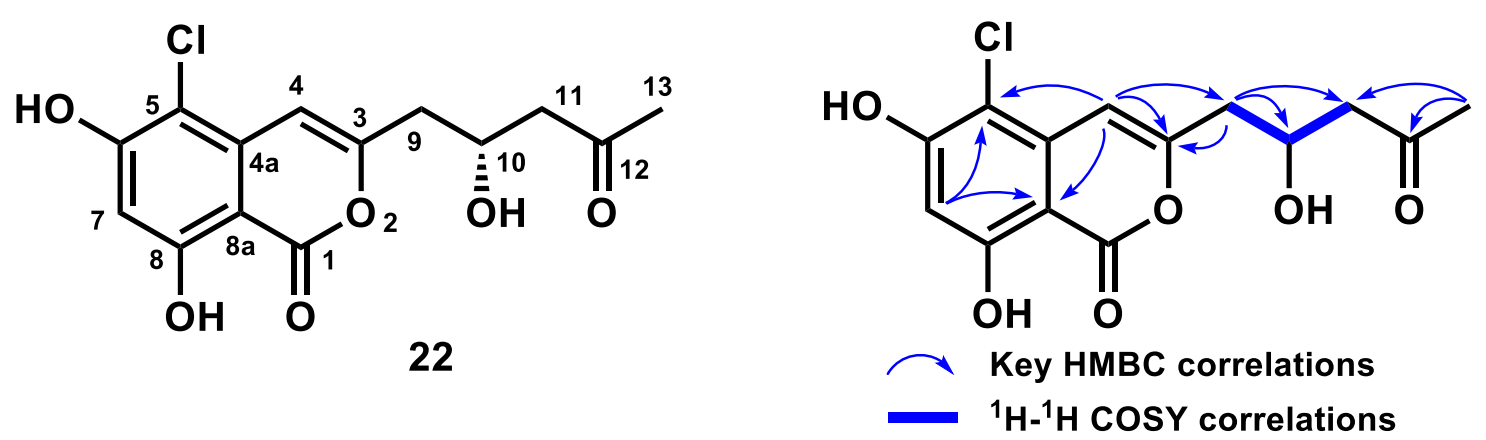

${ }^{1} \mathrm{H}$ NMR spectrum (500 MHz), ${ }^{13} \mathrm{C}$ NMR spectrum (125 MHz), $\mathrm{CD}_{3} \mathrm{OD}$

\begin{tabular}{c|ll}
\hline & \multicolumn{2}{|c}{22} \\
Position & $\delta_{\mathrm{H}}(J$ in Hz$)$ & $\delta_{\mathrm{C}}$, type \\
\hline 1 & & $167.2, \mathrm{C}$ \\
3 & & $157.1, \mathrm{C}$ \\
4 & $6.77, \mathrm{~s}$ & $103.7, \mathrm{CH}$ \\
$4 \mathrm{a}$ & & $137.6, \mathrm{C}$ \\
5 & & $107.2, \mathrm{C}$ \\
6 & & $162.6, \mathrm{C}$ \\
7 & $6.47, \mathrm{~s}$ & $103.0, \mathrm{CH}$ \\
8 & & $163.1, \mathrm{C}$ \\
$8 \mathrm{a}$ & & $100.4, \mathrm{C}$ \\
9 & $2.66, \mathrm{dd}(8.2,14.4) ; 2.76$, & $42.2, \mathrm{CH}_{2}$ \\
& $\mathrm{dd}(4.7,14.5)$ & \\
10 & $4.46, \mathrm{~m}$ & $66.4, \mathrm{CH}^{2}$ \\
11 & $2.72, \mathrm{~d}(6.3)$ & $51.1, \mathrm{CH}_{2}$ \\
12 & & $209.7, \mathrm{C}$ \\
13 & $2.19, \mathrm{~s}$ & $30.7, \mathrm{CH}_{3}$ \\
\hline
\end{tabular}

NMR spectrum (500 MHz) for ${ }^{1} \mathrm{H}$, NMR spectrum $(125 \mathrm{MHz})$ for ${ }^{13} \mathrm{C}, \mathrm{CD}_{3} \mathrm{OD}$, “m” means overlapped or multiple with other signals. Chemical shifts are reported in ppm.

HRMS (ESI, $\mathrm{M}+\mathrm{H}^{+}$) $m / z$ calculated for $\mathrm{C}_{14} \mathrm{H}_{14} \mathrm{ClO}_{6}{ }^{+}$313.0473; found 313.0472 .

$$
[\alpha]_{\mathrm{D}}^{24.1}+21.0^{\circ}(c 0.1, \mathrm{MeOH}) \text {. }
$$


Table S23. Spectroscopic data of compound $\mathbf{2 3}$

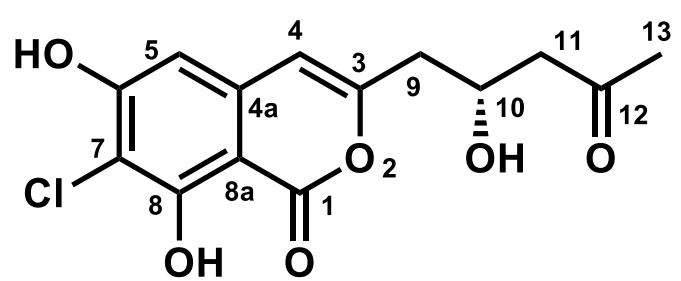

23

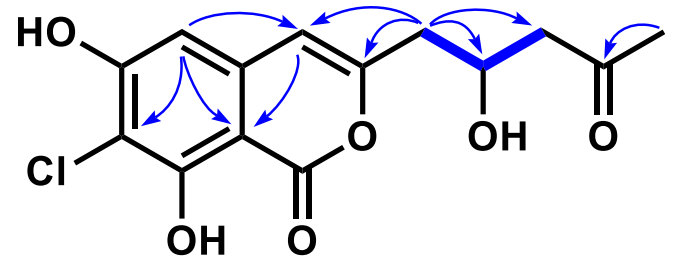

$\frown$ Key HMBC correlations

- ${ }^{1} \mathrm{H}-{ }^{1} \mathrm{H}$ COSY correlations

${ }^{1} \mathrm{H}$ NMR spectrum (500 MHz), ${ }^{13} \mathrm{C}$ NMR spectrum (125 MHz), $\mathrm{CD}_{3} \mathrm{OD}$

\begin{tabular}{c|ll}
\hline & \multicolumn{2}{|c}{23} \\
Position & $\delta_{\mathrm{H}}(J$ in $\mathrm{Hz})$ & $\delta_{\mathrm{C}}$, type \\
\hline 1 & & $167.6, \mathrm{C}$ \\
3 & & $155.9, \mathrm{C}$ \\
4 & $6.43, \mathrm{~s}$ & $107.0, \mathrm{CH}$ \\
$4 \mathrm{a}$ & & $138.4, \mathrm{C}$ \\
5 & $6.48, \mathrm{~s}$ & $103.7, \mathrm{CH}$ \\
6 & & $160.0, \mathrm{C}$ \\
7 & & $107.9, \mathrm{C}$ \\
8 & & $163.2, \mathrm{C}$ \\
$8 \mathrm{a}$ & & $100.2, \mathrm{C}$ \\
9 & $2.64, \mathrm{~m} ; 2.73, \mathrm{~m}$ & $41.9, \mathrm{CH} 2$ \\
10 & $4.47, \mathrm{~m}$ & $66.3, \mathrm{CH}$ \\
11 & $2.74, \mathrm{~m}$ & $51.0, \mathrm{CH}_{2}$ \\
12 & & $209.7, \mathrm{C}$ \\
13 & $2.21, \mathrm{~s}$ & $30.7, \mathrm{CH}_{3}$ \\
\hline
\end{tabular}

NMR spectrum (500 MHz) for ${ }^{1} \mathrm{H}$, NMR spectrum $\left(125 \mathrm{MHz}\right.$ ) for ${ }^{13} \mathrm{C}, \mathrm{CD}_{3} \mathrm{OD}$, “m” means overlapped or multiple with other signals. Chemical shifts are reported in ppm.

HRMS (ESI, $\mathrm{M}+\mathrm{H}^{+}$) $m / z$ calculated for $\mathrm{C}_{14} \mathrm{H}_{14} \mathrm{ClO}_{6}{ }^{+}$313.0473; found 313.0471 .

$$
[\alpha]_{\mathrm{D}}^{24.1}+17.0^{\circ}(c 0.1, \mathrm{MeOH})
$$


Tryptophan Halogenases

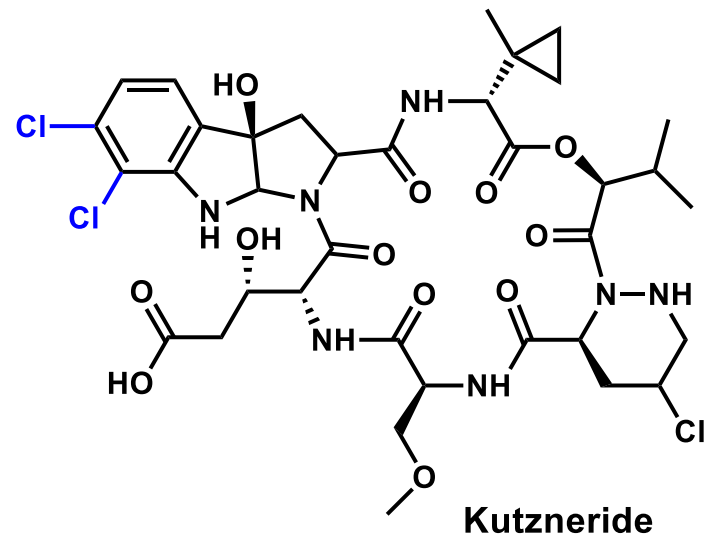<smiles>CNC(=O)[C@]1(O)C(=O)c2c([nH]c3ccc(Cl)cc23)C2=C(OC)c3ccccc3C21</smiles>

Cladoniamide D<smiles>O=[N+]([O-])c1c(Cl)cccc1-c1c[nH]cc1Cl</smiles>

Pyrrolnitrin

Pyrrole Halogenases<smiles>Oc1c(Cl)cc(Cl)cc1-c1[nH]c(Cl)c(Cl)c1Cl</smiles>

Pentachloropseudilin<smiles>O=C1c2c(cc(O)c(C3CCc4cccn43)c2O)-c2cc(Cl)c(Cl)n21</smiles>

Chlorizidine<smiles>O=C(SPC#P)c1ccc[nH]1</smiles>

pyrrole-2-carboxy-S-Bmp1

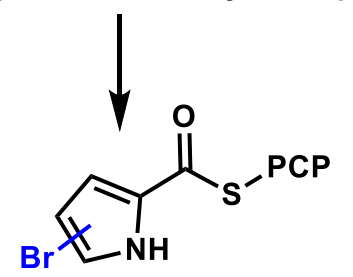

Phenolic halogenases<smiles>CC1C[C@@H]2O[C@@H](/C=C\C=C/C(=O)Cc3c(Cl)c(O)cc(O)c3C2=O)O1</smiles>

Radicicol<smiles>C[C@H]1Cc2c(Cl)cc(C(=O)N[C@@H](Cc3ccccc3)C(=O)O)c(O)c2C(=O)O1</smiles>

Ochratoxin B<smiles>COC1=CC(=O)C[C@H](C)[C@]12Oc1c(Cl)c(OC)cc(OC)c1C2=O</smiles>

Griseofulvin

Figure S1. Natural products with halogenation of the aromatic C-H by flavin-dependent halogenases. ${ }^{[13]}$ 

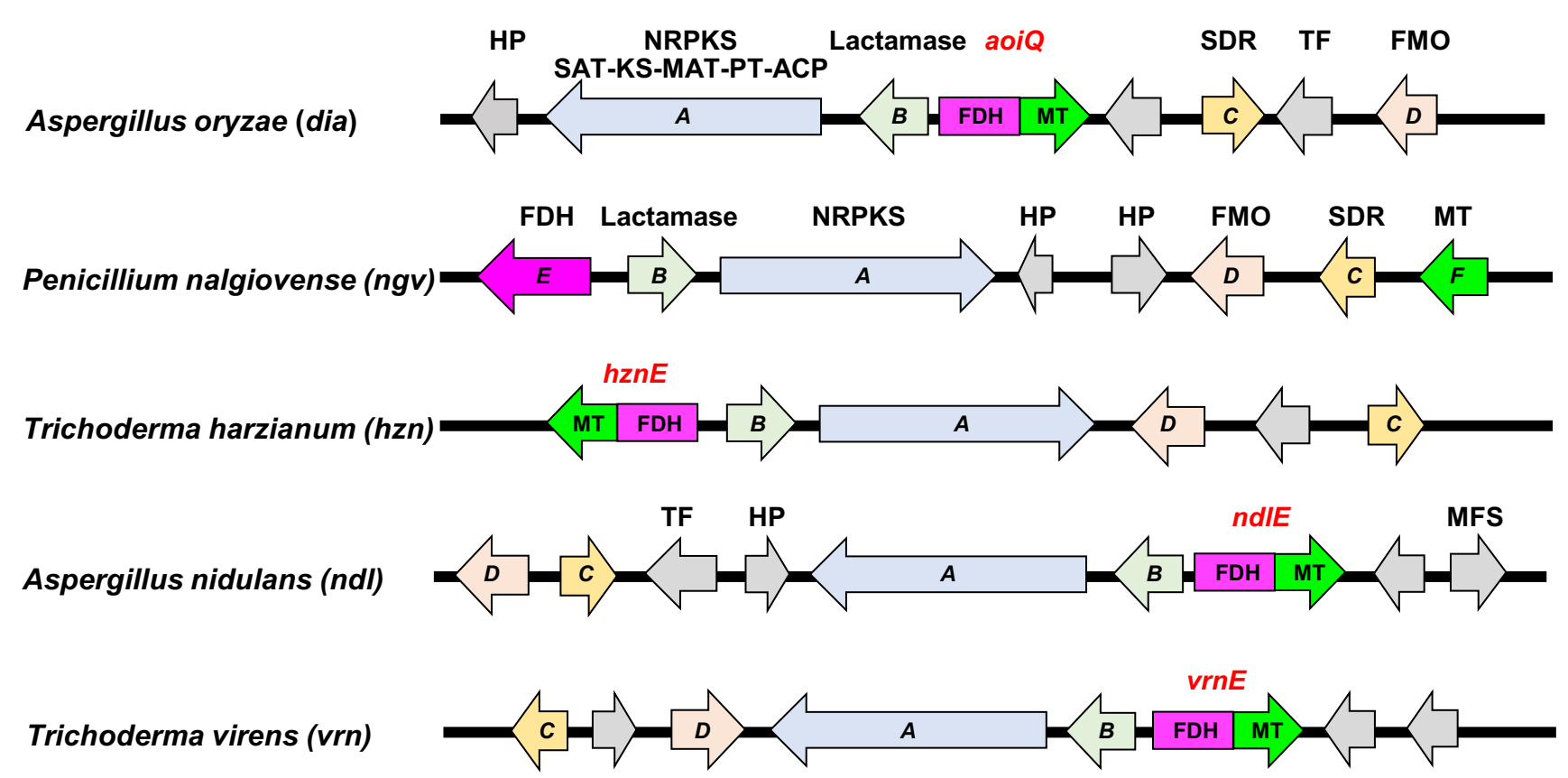

Figure S2. Homologous biosynthetic gene clusters of dia from NCBI database. aoiQ is conserved in many other fungi along with $\operatorname{diaA}$. 
In S. cerevisiae

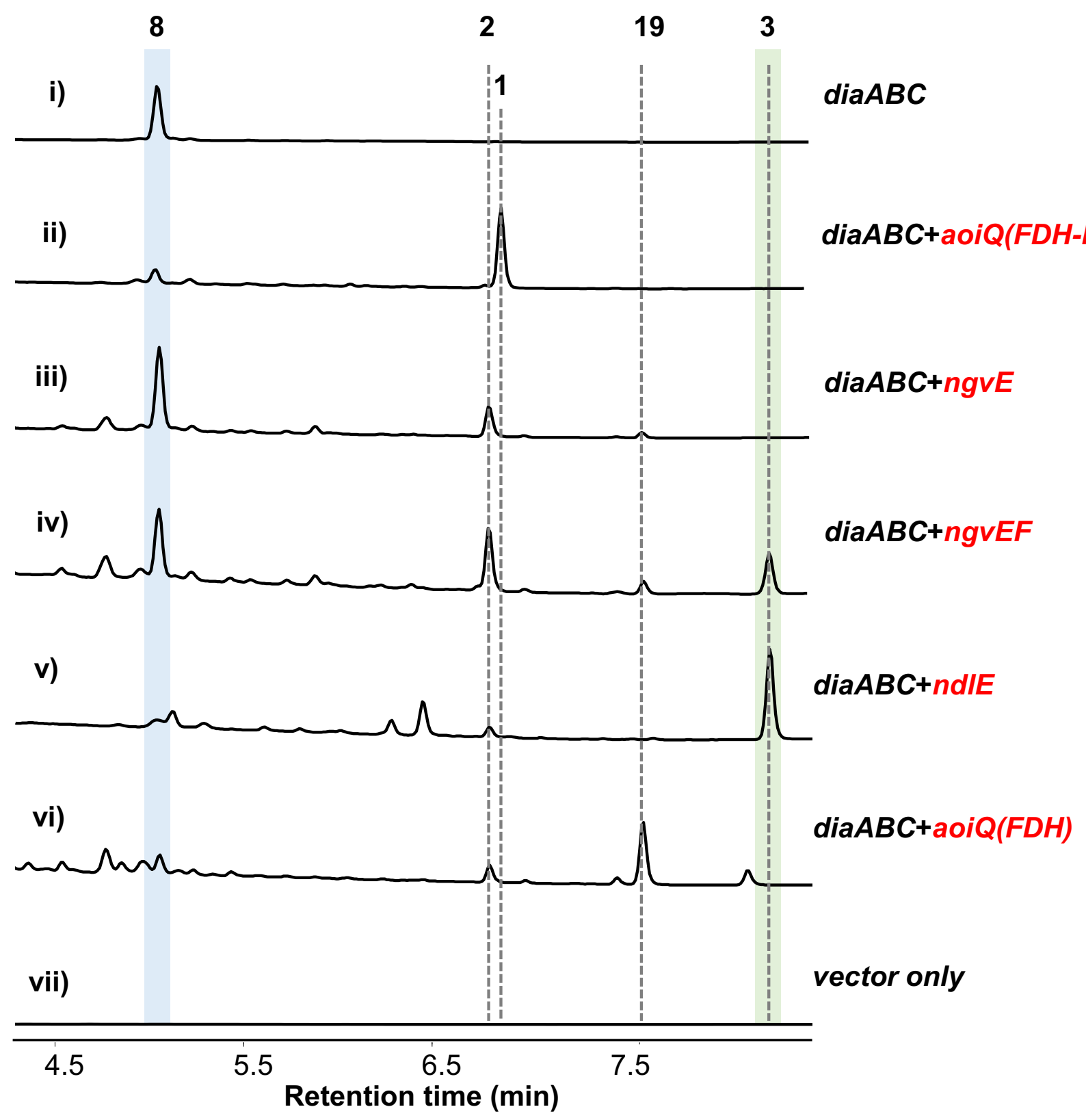

Figure S3. Functional analysis of AoiQ homologs from different fungi in S. cerevisiae BJ5464. Dichlorinated orthosporins 1, 2 or 3 were present as major peak when in $S$. cerevisiae expressing diaABC with combination of different AoiQ homologs (trace iii-v). 
In S. cerevisiae $\quad \lambda=240 \mathrm{~nm}$

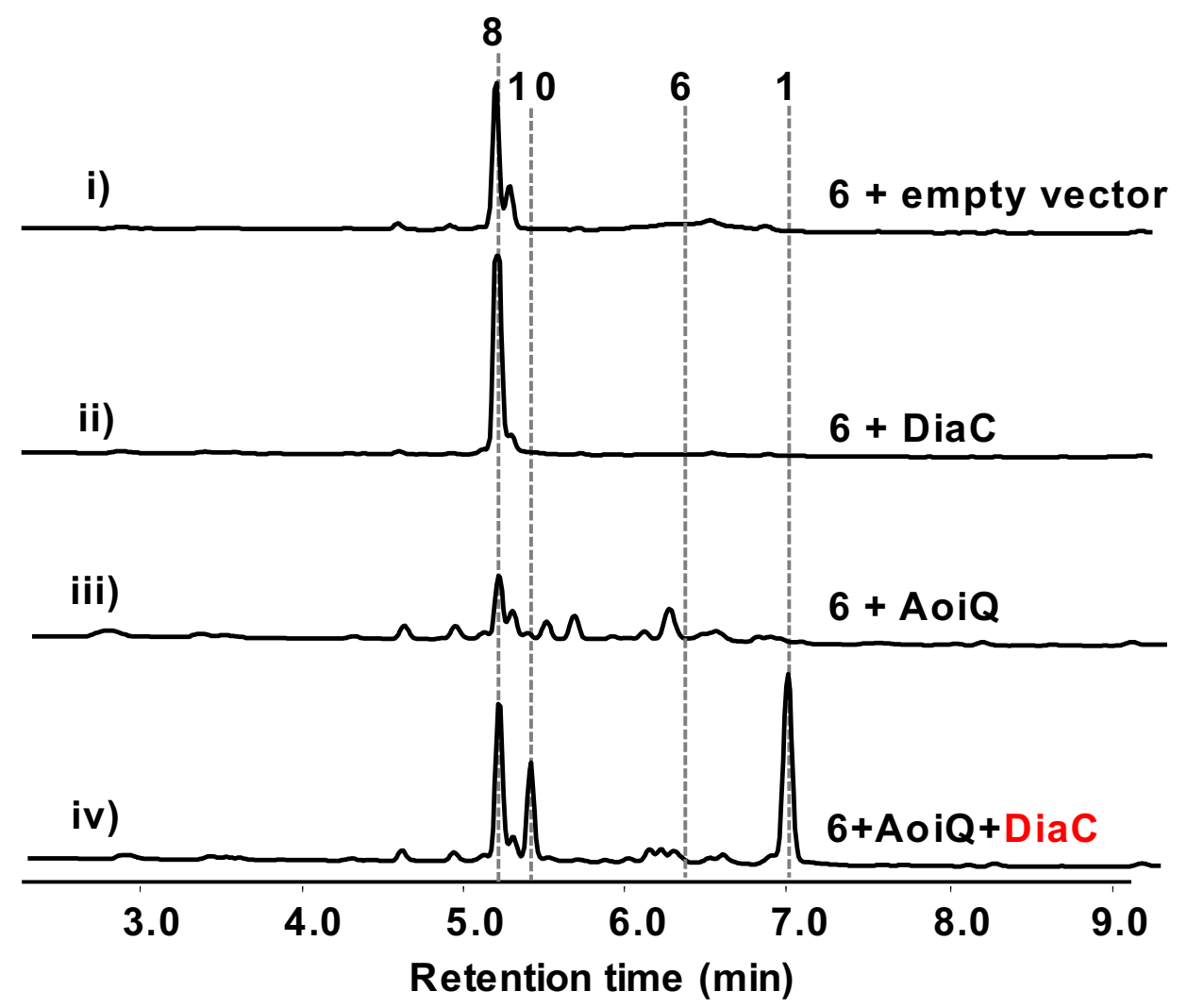

Figure S4. LC-MS analysis of extracts from feeding 6 to S. cerevisiae expressing AoiQ with or without DiaC. Yeast cultures fed with $500 \mu \mathrm{M} 6$ was extracted after 12 hours. 


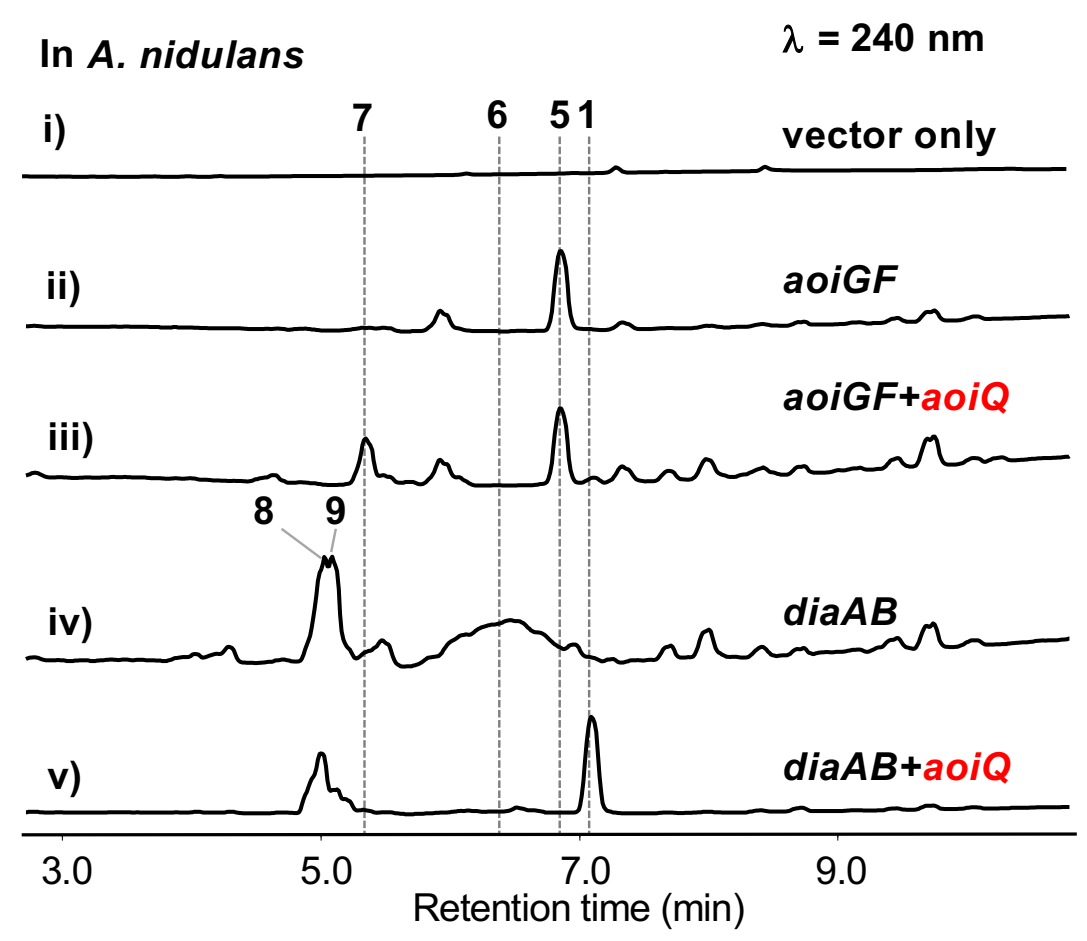

Figure S5. LC-MS analysis of products profiles of heterologous expression of aoi and dia genes in A. nidulans. For each trace, the gene indicated in red represents addition from the previous construct. 


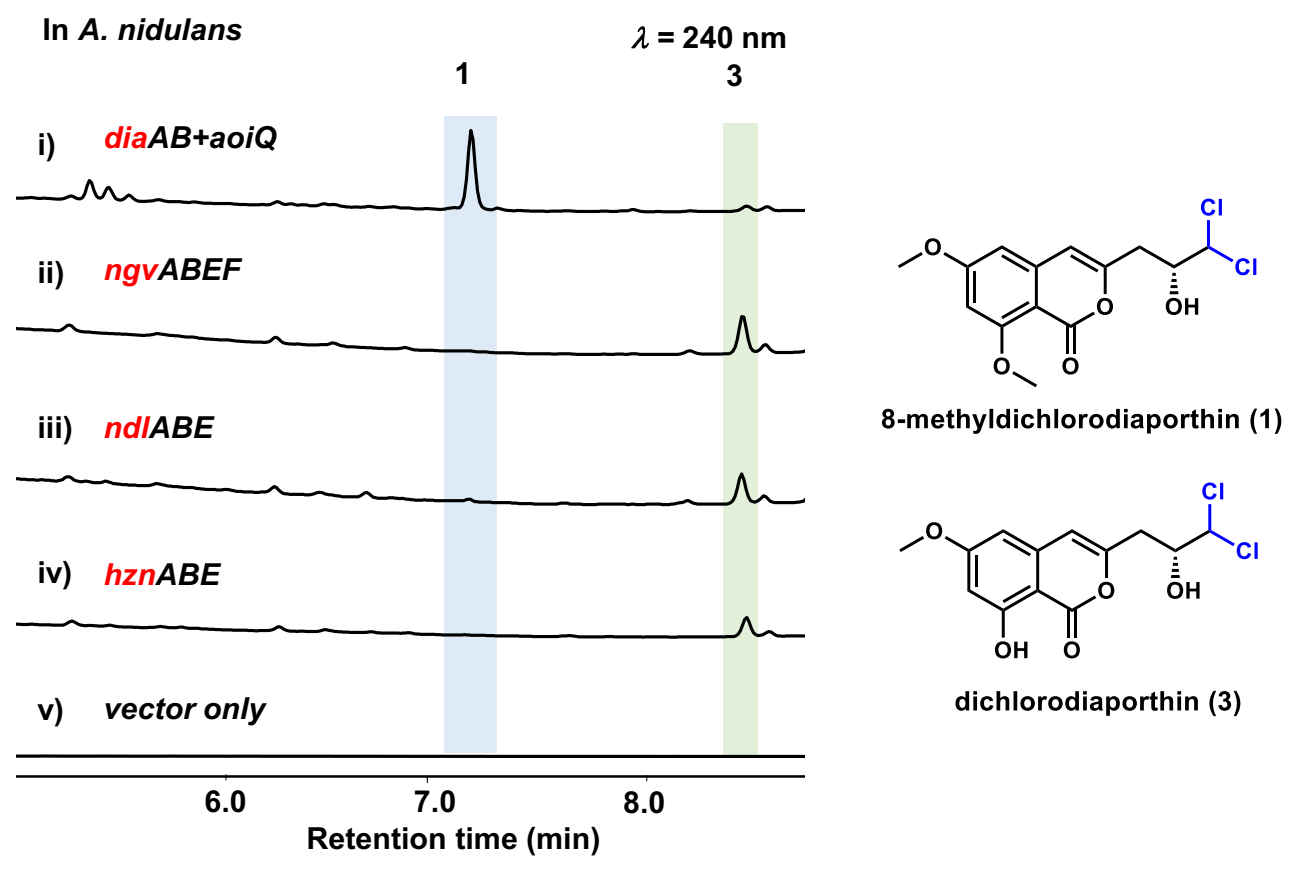

Figure S6. LC/MS profiles of the metabolites from $A$. nidulans transformants expressing dia and homologous clusters $(n g v, n d l, h z n)$. Overexpression dia cluster in A. nidulans successfully yielded 8-methyldichlorodiaporthin (1) whereas homologous clusters $n g v, n d l$ and $h z n$ were responsible for dichlorodiaporthin (3) formation (trace iiiv). 


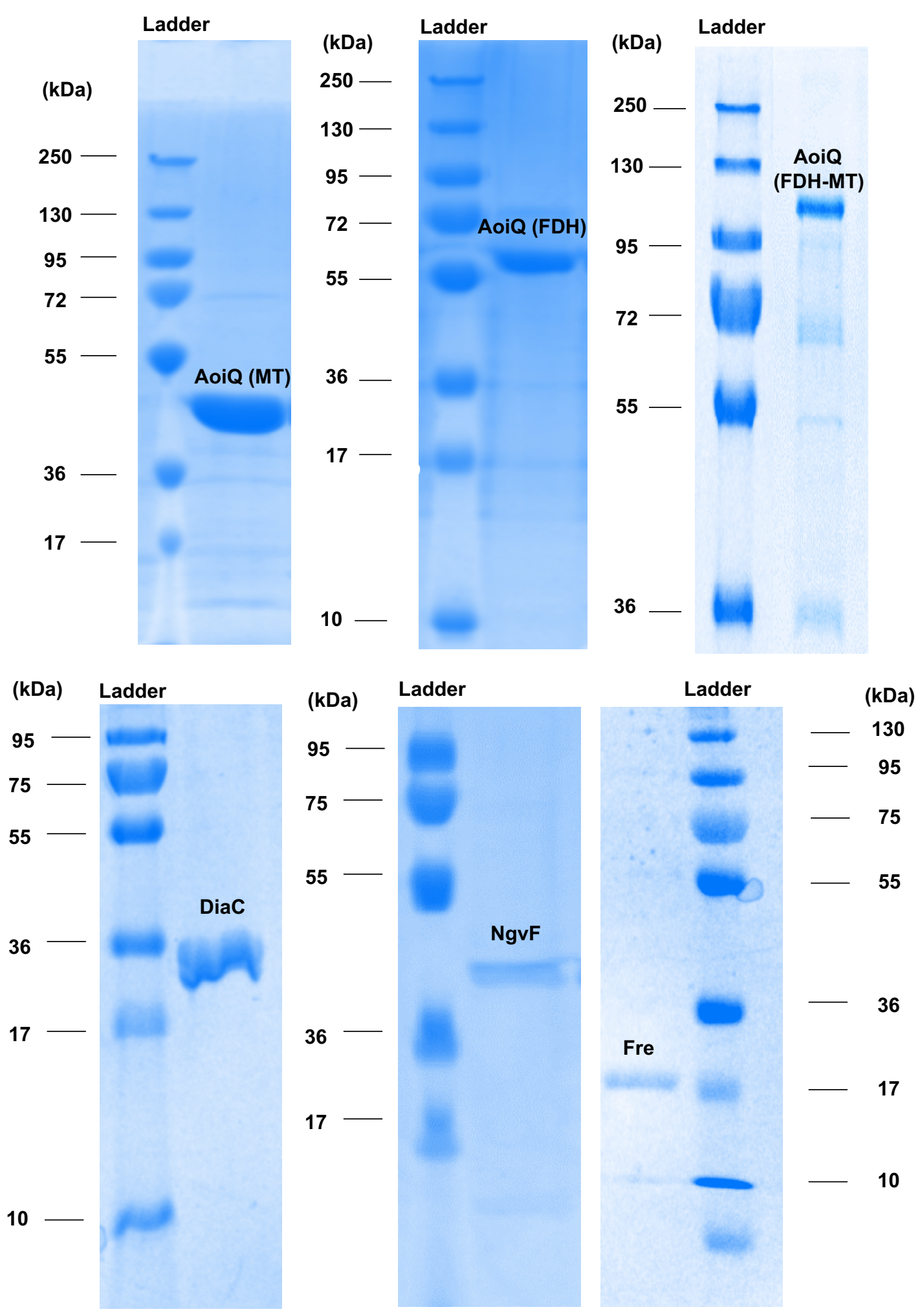

Figure S7. SDS-PAGE gels of purified proteins used in this study. Expected molecular weights of AoiQ (MT), AoiQ (FDH), AoiQ (FDH-MT), DiaC, NgvF, Fre are 42 kDa, 61 kDa, 111 kDa, 33 kDa, 43 kDa and 26 kDa, respectively. 
A

EIC $[M+H]^{+}=786$

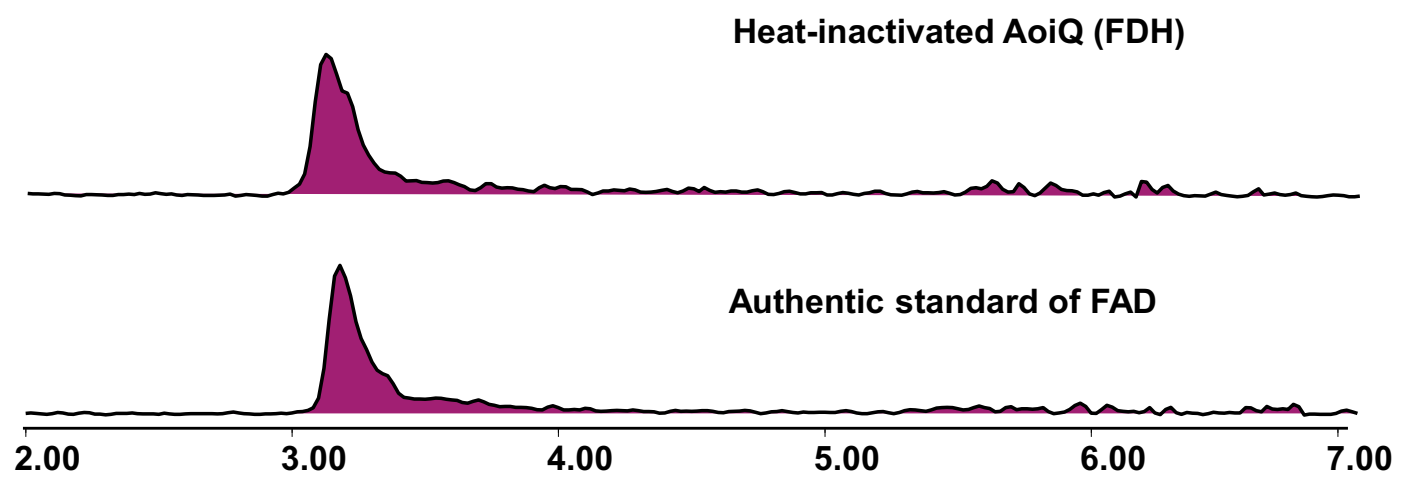

B

C
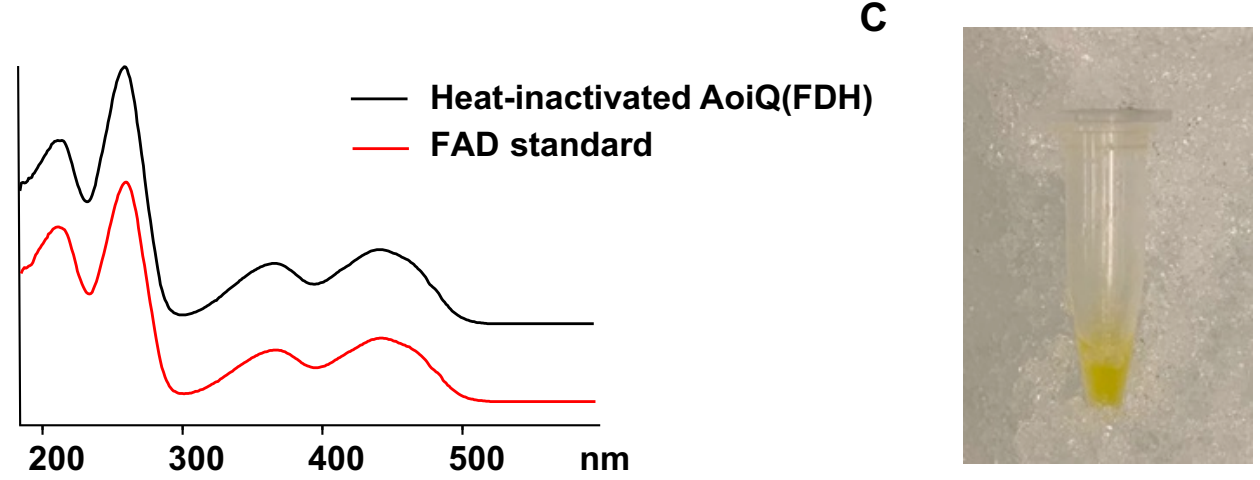

Figure S8. Characterization of AoiQ (FDH) as FAD binding protein by LC/MS analysis. A standard solution of FAD was used to establish a standard curve based on LC area at $\lambda=446 \mathrm{~nm}$. A solution $(30 \mu \mathrm{L})$ containing $100 \mu \mathrm{M}$ AoiQ (FDH) purified from yeast was denatured by heating at $100{ }^{\circ} \mathrm{C}$ for $15 \mathrm{~min}$. After centrifugation, the supernatant was mixed with equal volume of $\mathrm{MeOH}$ and subjected to the LC/MS analysis. The supernatant of boiled AoiQ (FDH) showed FAD is copurified with AoiQ (FDH). Using standard curve, the amount of FAD released from denatured AoiQ (FDH) corresponded to approximately $86 \%$ of the AoiQ (FDH) concentration. (A) The peak with the same retention time as the FAD standard was identified in the supernatant of denatured. (B) The characteristic UV absorption with max at $375 \mathrm{~nm}$ and $450 \mathrm{~nm}$ of this peak was also identical to that of the FAD standard. (C) Purified AoiQ (FDH) protein showed bright yellow, suggesting the existence of a prosthetic group. 

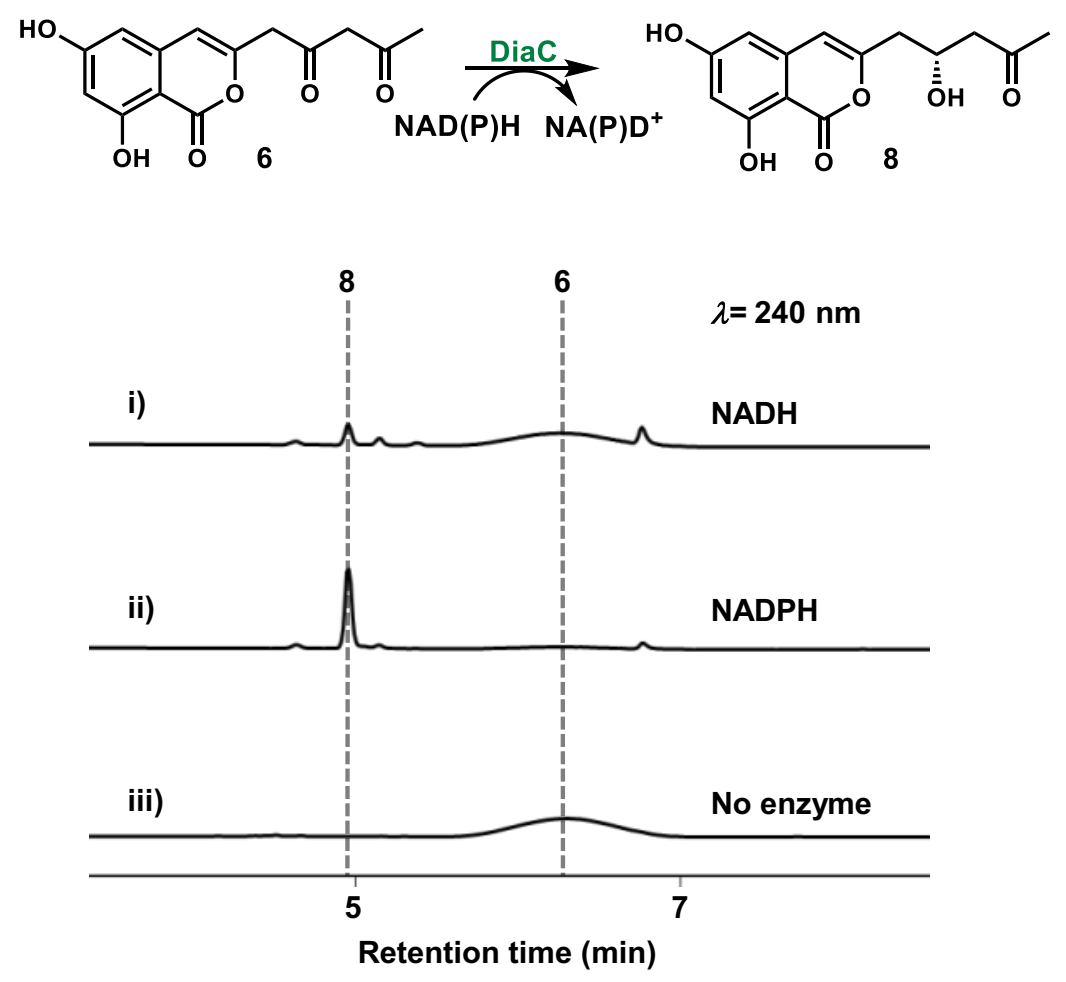

Figure S9. In vitro DiaC cofactor requirements with compound $\mathbf{6}$ as the substrate. The reaction mixture contained $10 \mu \mathrm{M} \mathrm{DiaC}, 500 \mu \mathrm{M}$ compound 6, with (i) $1 \mathrm{mM} \mathrm{NADPH}$; (ii) $1 \mathrm{mM} \mathrm{NADH}$. The reactions were incubated at $30{ }^{\circ} \mathrm{C}$ for 3 hours, quenched with equal amount of $\mathrm{MeCN}$ to terminate the reaction. 


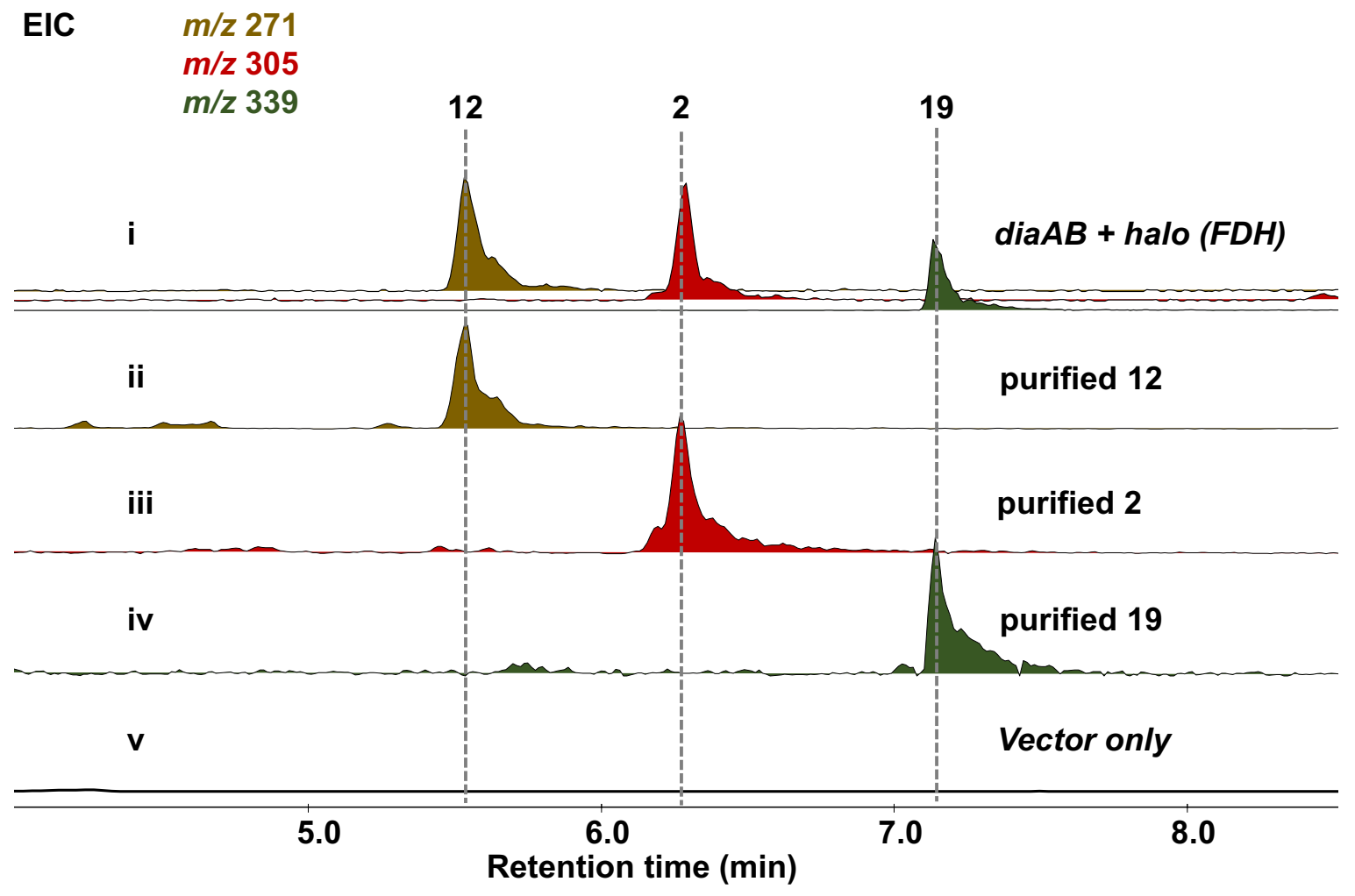

Figure S10. LC/MS analysis of extracts from A. nidulans expressing diaAB and dissected aoiQ (FDH) (trace i). The purified 2 and 12 has identical NMR spectra to the reported data (Tables S5 and S14). The extracted ion chromatograms (EICs) were extracted at $m / z 305[\mathrm{M}+\mathrm{H}]^{+}$for 2, $m / z 271[\mathrm{M}+\mathrm{H}]^{+}$for 12, and $m / z 399[\mathrm{M}+$ $\mathrm{H}]^{+}$for 19. 


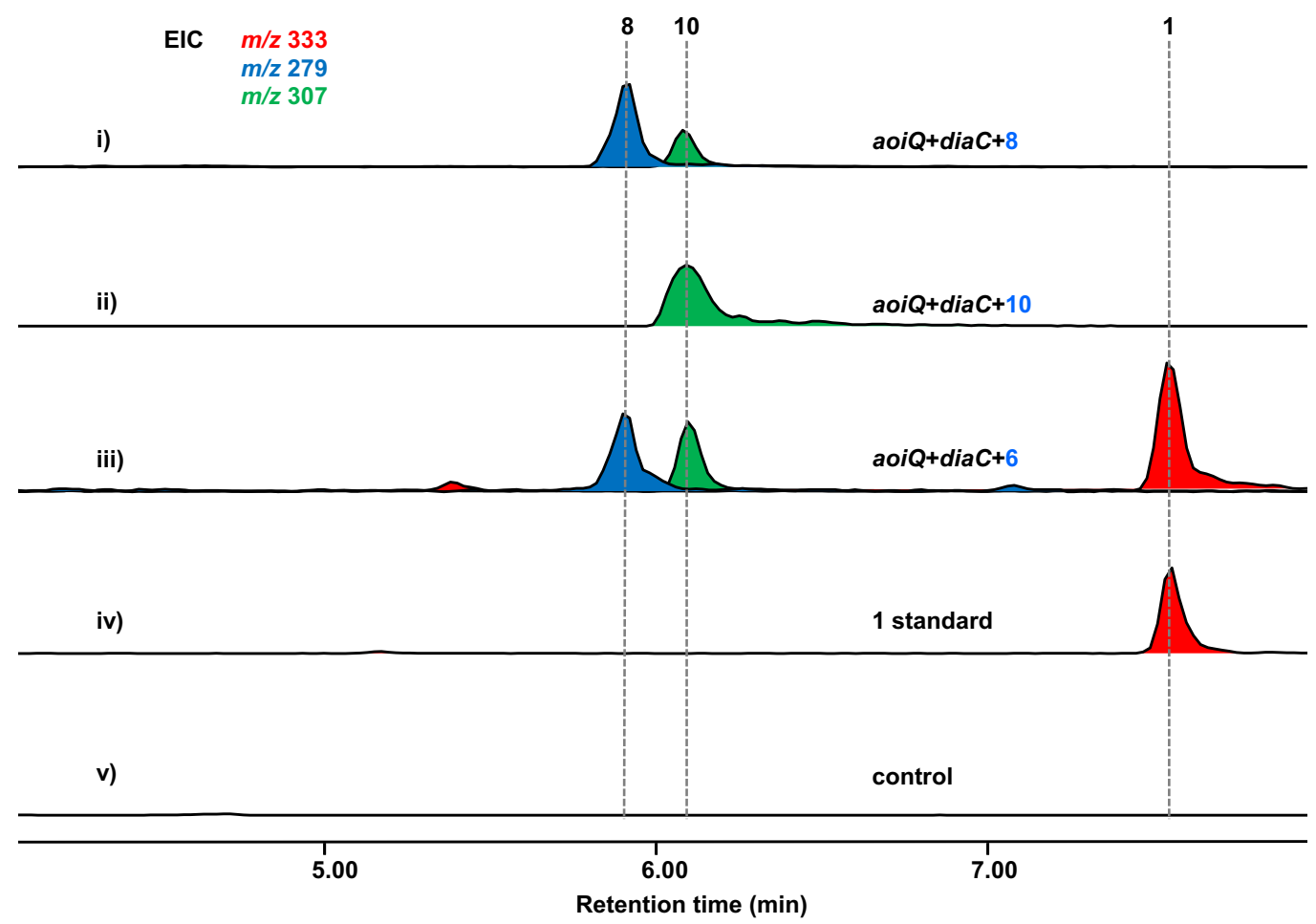

Figure S11. LC/MS analysis of extracts from feeding compound 6, 8, and 10 to $S$. cerevisiae expressing aoiQ with diaC genes. Yeast cultures fed with $500 \mu \mathrm{M}$ compound was extracted after 12 hours and concentrated. 1 was observed only when $\mathbf{6}$ was fed to yeast expressing aoiQ and $\operatorname{dia} C$, which indicates that $\mathbf{8}$ and $\mathbf{1 0}$ were shunt. EIC, extracted ion chromatogram. The extracted ion chromatograms (EICs) were extracted at $m / z 333[\mathrm{M}+\mathrm{H}]^{+}$ for $\mathbf{1}, m / z 279[\mathrm{M}+\mathrm{H}]^{+}$for $\mathbf{8}$, and $m / z 307[\mathrm{M}+\mathrm{H}]^{+}$for $\mathbf{1 0}$. 

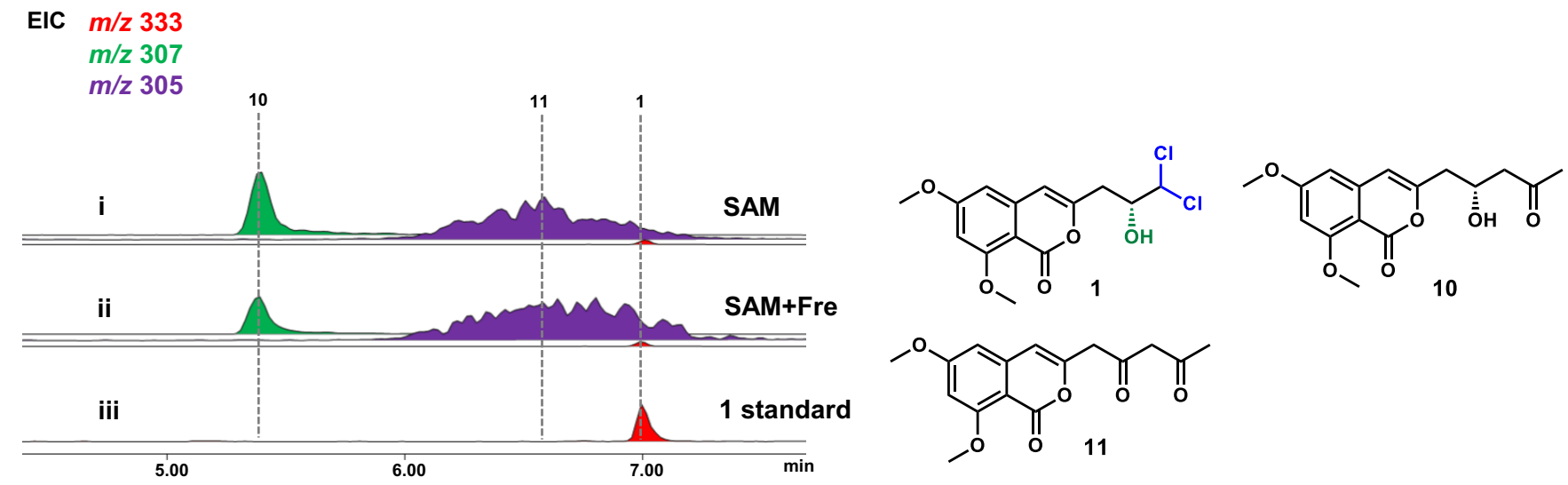

Figure S12. LC/MS analysis of enzymatic chlorination of AoiQ (FDH-MT). The reaction mixture contained 5 $\mu \mathrm{M}$ DiaC, $50 \mu \mathrm{M}$ FAD, $150 \mu \mathrm{M}$ AoiQ (FDH-MT), $10 \mathrm{mM}$ NADH, $25 \mathrm{mM} \mathrm{NaCl}, 50 \mathrm{mM}$ phosphate buffer (PH 6.7), $500 \mu \mathrm{M}$ compound 6 with (i) $1 \mathrm{mM}$ SAM; (ii) $1 \mathrm{mM} \mathrm{SAM}+10 \mu \mathrm{M}$ Fre. The reactions were incubated at $30{ }^{\circ} \mathrm{C}$ for 3 hours, quenched with equal amount of $\mathrm{MeCN}$ to terminate reaction. The extracted ion chromatograms (EICs) were extracted at $m / z 333[\mathrm{M}+\mathrm{H}]^{+}$for $\mathbf{1}, \mathrm{m} / z 305[\mathrm{M}+\mathrm{H}]^{+}$for 11, and $m / z 307[\mathrm{M}+\mathrm{H}]^{+}$for $\mathbf{1 0}$. 


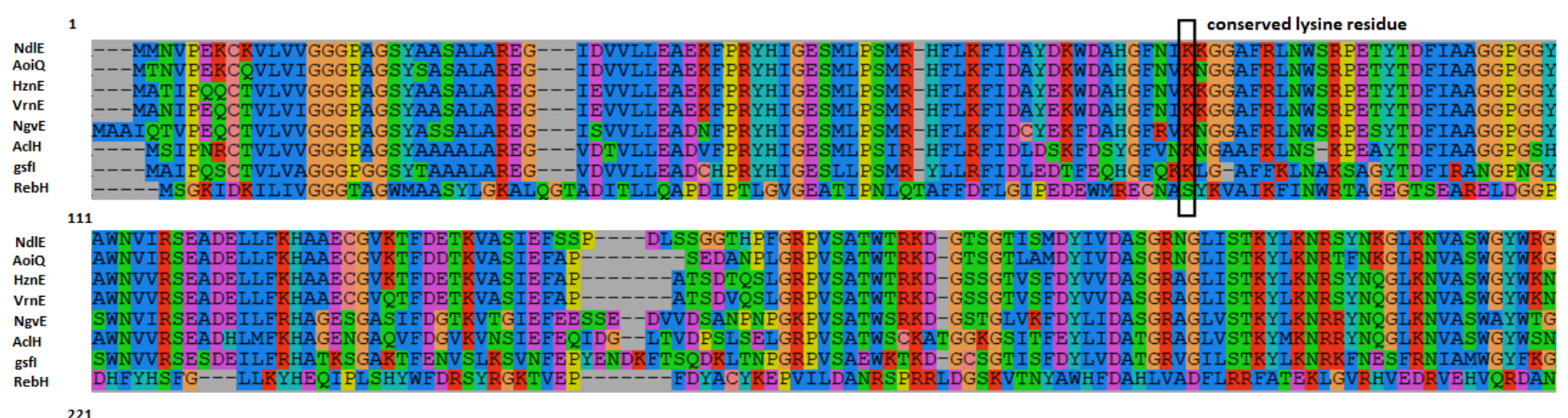
221

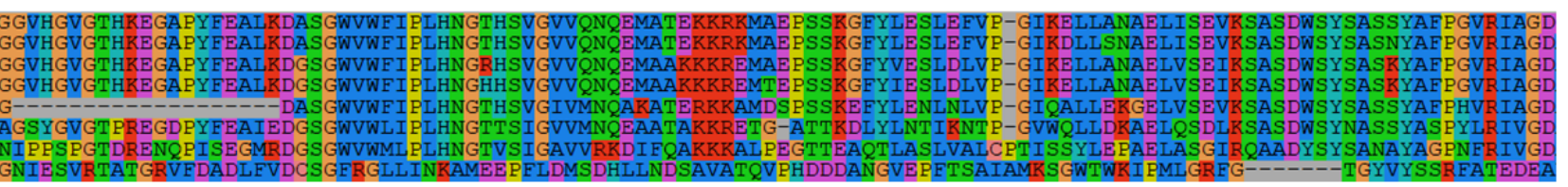
331

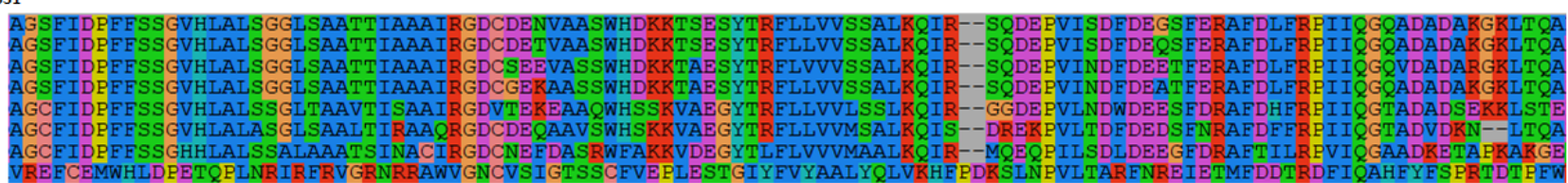
441

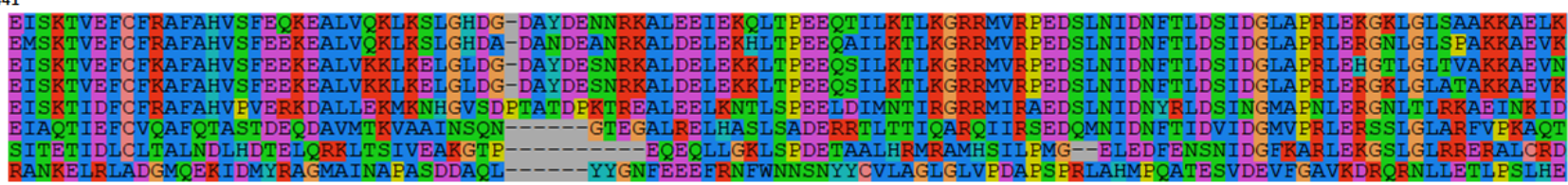
551

$\nabla$ AoiQ (FDH-MT) was dissected

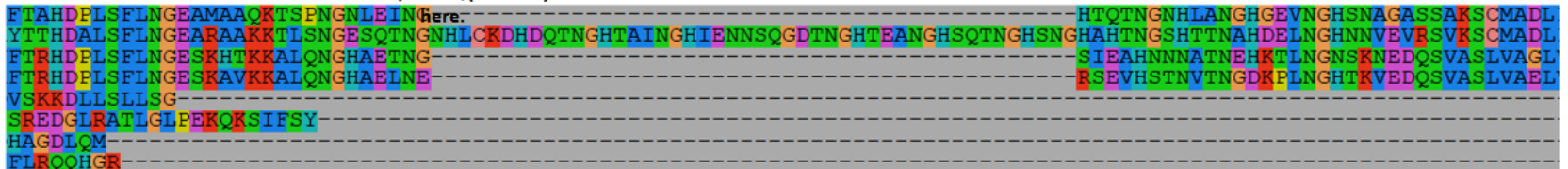

661

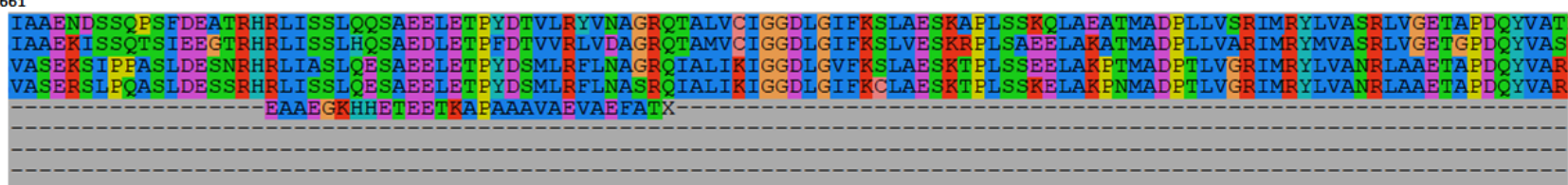

Figure S13. The multiple sequence alignments of AoiQ, homologs of AoiQ (NdlE, HznE, VrnE, NgvE), reported three fungal origin FDHs (AclH,${ }^{14}$ gsfI, ${ }^{15}$ ) and bacterial FDH (RebH). ${ }^{16}$ The alignment was generated by the ClustalW program. Previous study of FDH suggested that the conserved lysine residue is important for capturing the hypohalous acid intermediate to control the regioselectivity of halogenation, ${ }^{17}$ and the lysine residue is also conserved in AoiQ and its homolog. Note: the place where AoiQ was dissected labeled with black triangle. Gsfl, from Penicillium aethiopicum. AclH, from Aspergillus oryzae. RebH, from Lechevalieria aerocolonigenes. 

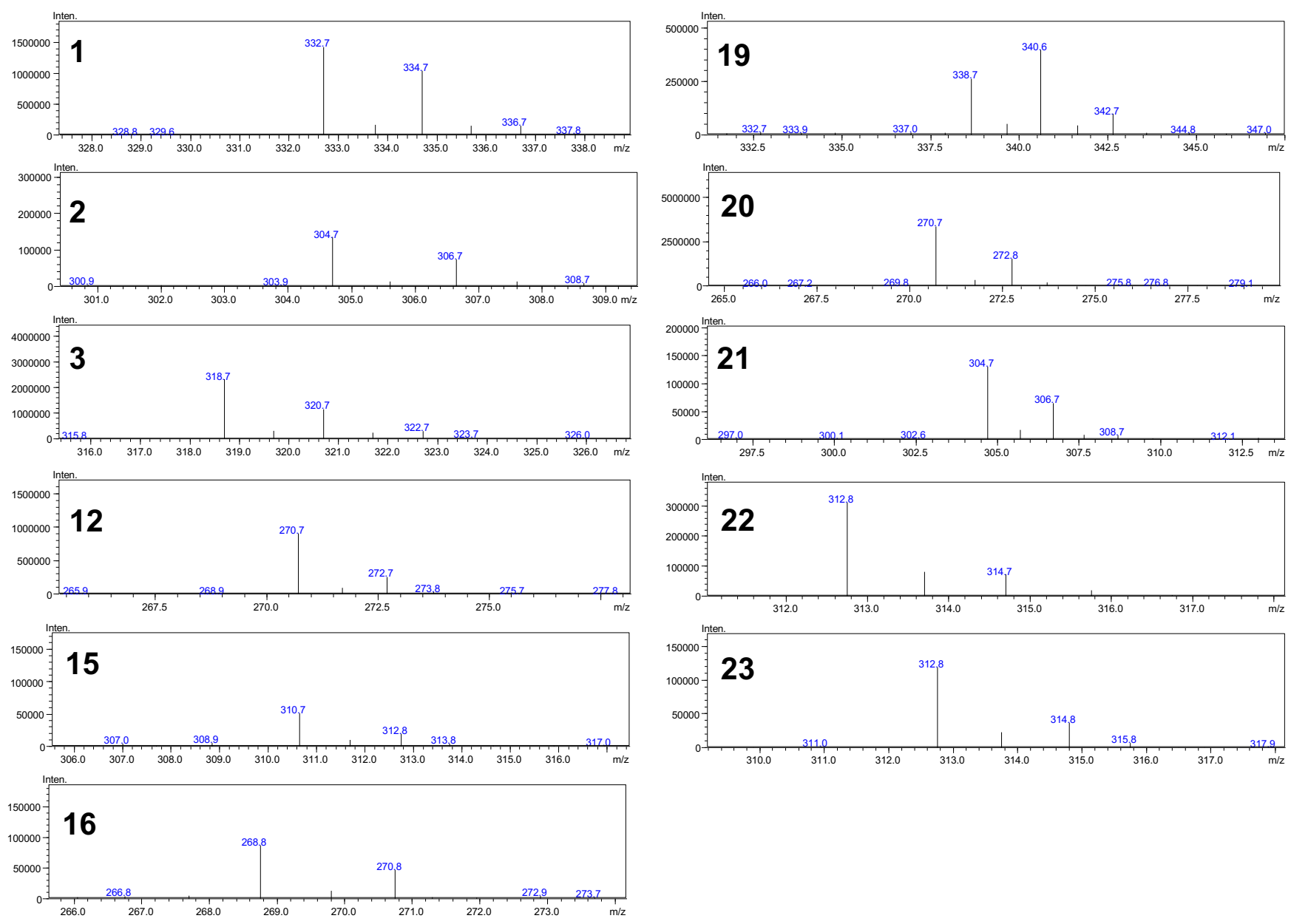

Figure S14. HPLC-MS profiles (extracted mass traces) of compounds 1, 2, 3, 12, 15, 16, 19, 20, 21, 22 and 23. 


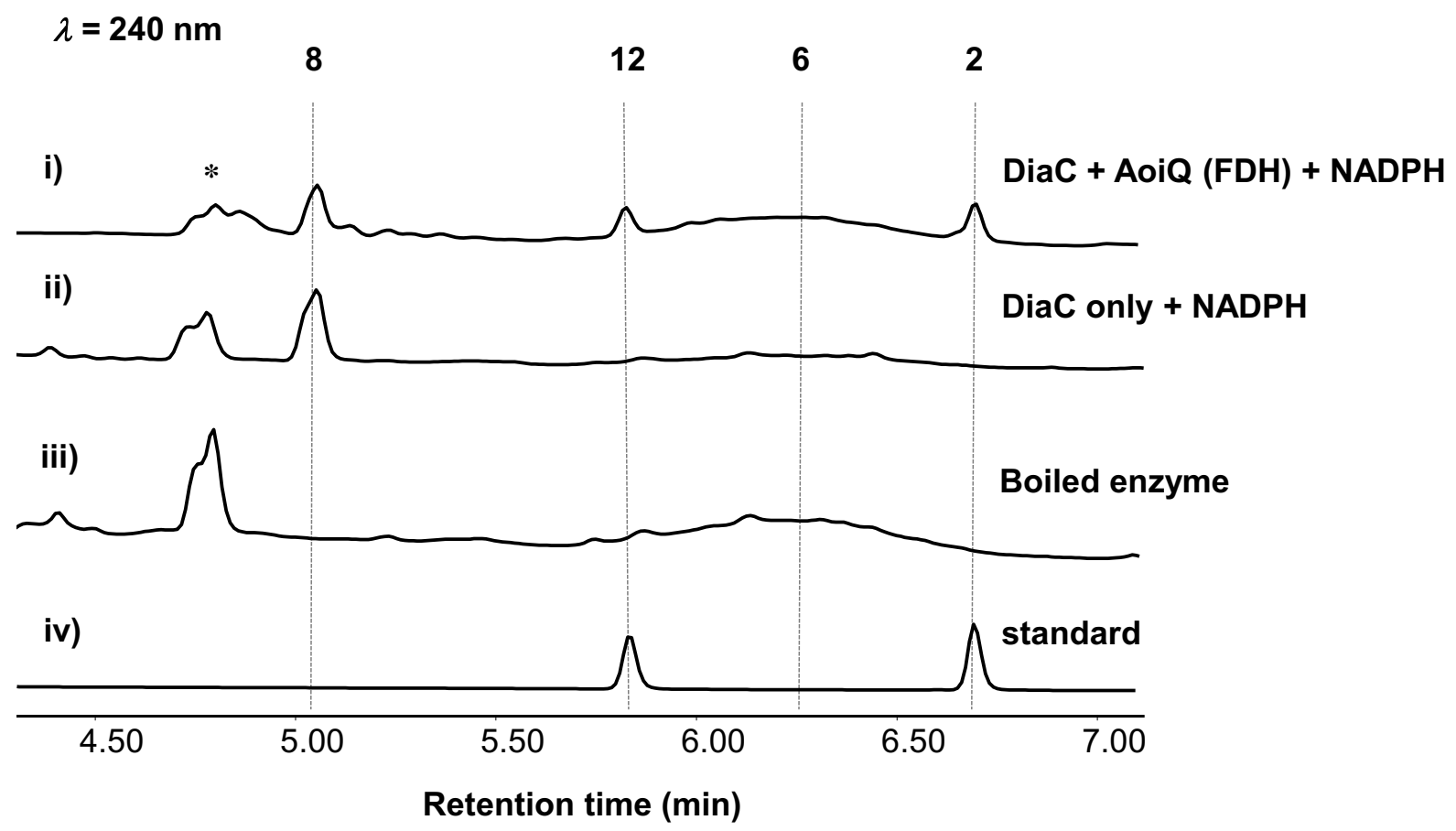

Figure S15. LC/MS analysis of enzymatic reaction of AoiQ (FDH) with compound $\mathbf{6}$ as substrate. Chlorinated Compounds 2 and 12 were also observed by using NADPH cofactor. The reaction mix contained $50 \mu \mathrm{M}$ FAD, 10 mM NADPH, $25 \mathrm{mM} \mathrm{NaCl}, 50 \mathrm{mM}$ phosphate buffer (PH 6.7) and $500 \mu \mathrm{M}$ compound 6 with i) $5 \mu \mathrm{M} \mathrm{DiaC}+$ $150 \mu \mathrm{M}$ AoiQ (FDH); ii) $5 \mu \mathrm{M}$ DiaC + heat-inactivated AoiQ (FDH); iii) boiled DiaC + boiled AoiQ (FDH); iv) standards for 2 and 12. The reactions were incubated at $30{ }^{\circ} \mathrm{C}$ for 3 hours, quenched with equal amount of $\mathrm{MeCN}$ to terminate the reaction. The peak labeled in * was proposed to be decomposition products in reaction condition. 


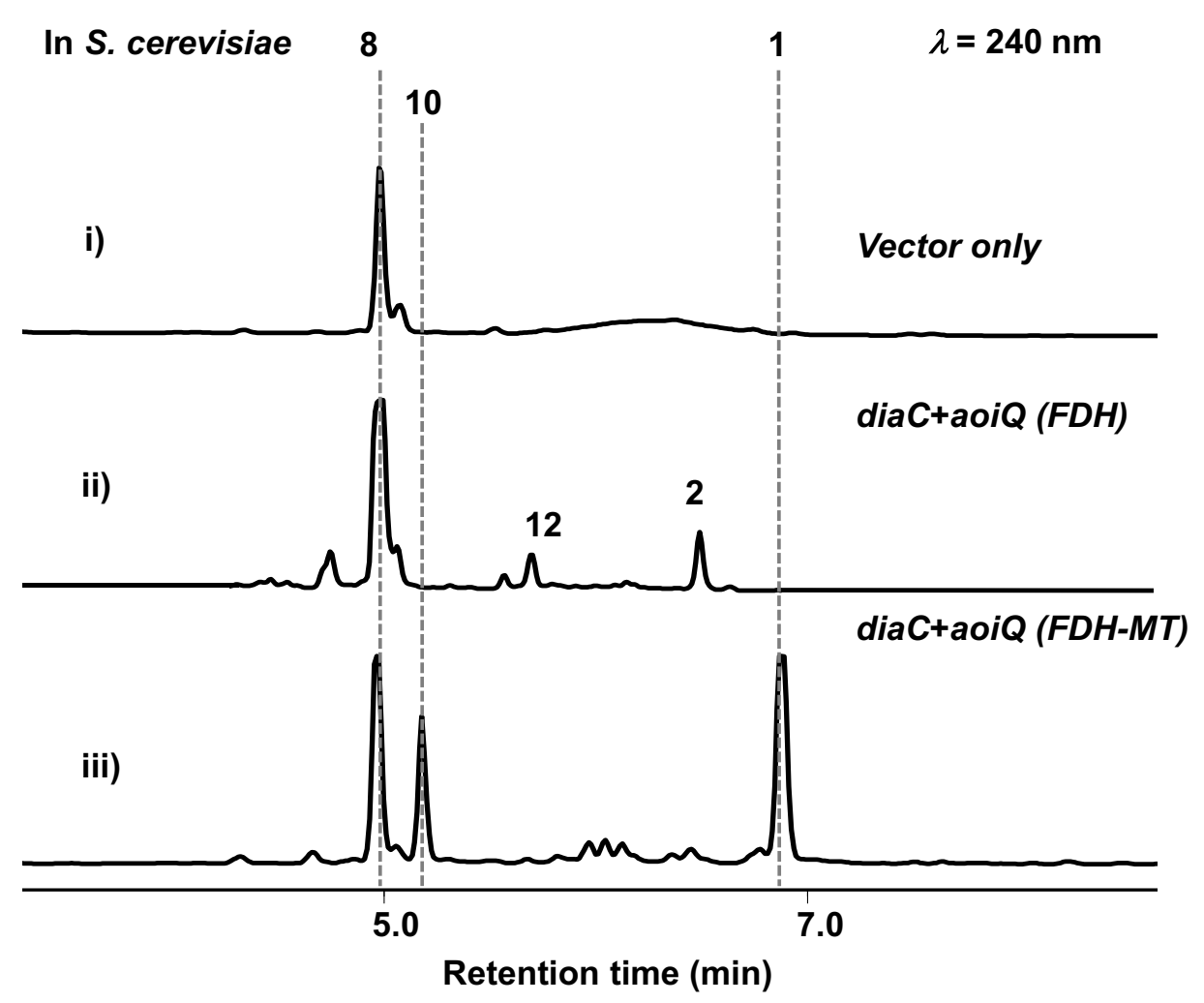

Figure S16. LC/MS analysis of extracts from feeding compound 6 to $S$. cerevisiae expressing i) empty vector; ii) diaC and dissected aoiQ (FDH); iii) diaC and dissected aoiQ (FDH-MT). Yeast cultures fed with $500 \mu \mathrm{M}$ compound 6 was extracted after 12 hours and concentrated. 


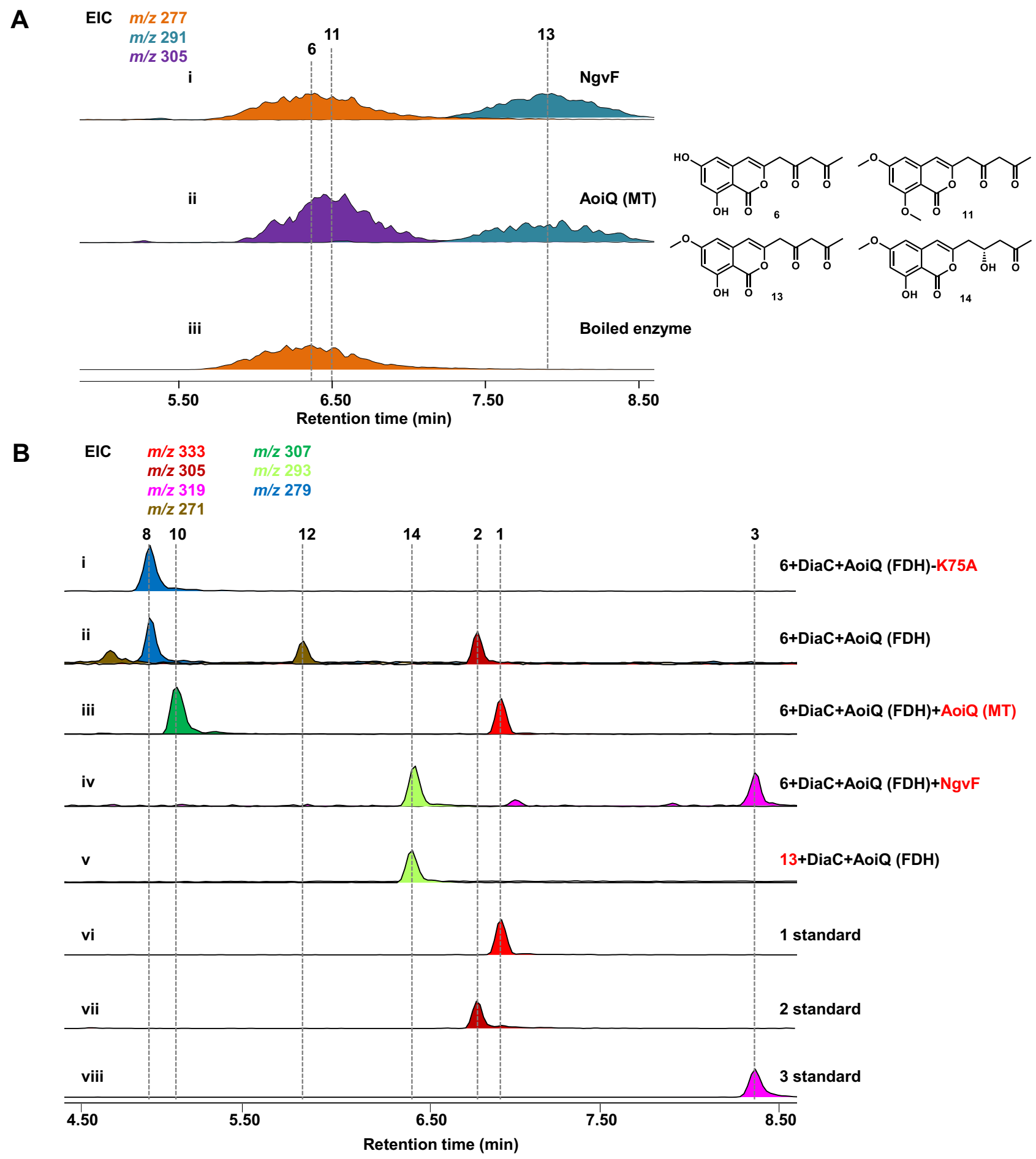

Figure S17. In vitro verification of MT domain involvement, and verification of lysine residue requirement for AoiQ (FDH) chlorination function. (A) In vitro AoiQ (MT) enzyme requirement for dimethylation or NgvF for monomethylation with compound 6 as substrate. The reaction mix contained $2 \mathrm{mM}$ SAM, $500 \mu \mathrm{M}$ compound $\mathbf{6}$, and $6 \mu \mathrm{M}$ AoiQ (MT) or NgvF. The reactions were incubated at $30^{\circ} \mathrm{C}$ for 1.5 hours, quenched with equal amount of $\mathrm{MeCN}$ to terminate reaction. (B) LC/MS analysis of one-pot enzymatic reaction of AoiQ (FDH) mutant with 
DiaC (trace i), and enzymatic reaction of AoiQ (FDH) with DiaC together with AoiQ (MT) domain or NgvF (trace iii-iv). The reaction mix contained $5 \mu \mathrm{M}$ DiaC, $50 \mu \mathrm{M} \mathrm{FAD}, 10 \mathrm{mM} \mathrm{NADH}, 25 \mathrm{mM} \mathrm{NaCl}, 50 \mathrm{mM}$ phosphate buffer (PH 6.7), 1 mM SAM, $500 \mu \mathrm{M}$ compound 6 or 13, and $150 \mu \mathrm{M}$ AoiQ (FDH) or mutant with $6 \mu \mathrm{M}$ AoiQ (MT) domain or $6 \mu \mathrm{M} \mathrm{NgvF}$. The reactions were incubated at $30^{\circ} \mathrm{C}$ for 3 hours, quenched with equal amount of $\mathrm{MeCN}$ to terminate reaction. The extracted ion chromatograms (EICs) were extracted at $m / z 333[\mathrm{M}+\mathrm{H}]^{+}$for $\mathbf{1}, \mathrm{m} / z 305$ $[\mathrm{M}+\mathrm{H}]^{+}$for $\mathbf{2}$ and 11, $m / z 319[\mathrm{M}+\mathrm{H}]^{+}$for $\mathbf{3}, \mathrm{m} / z 271[\mathrm{M}+\mathrm{H}]^{+}$for 12, $m / z 277[\mathrm{M}+\mathrm{H}]^{+}$for $\mathbf{6}, m / z 279[\mathrm{M}+\mathrm{H}]^{+}$ for $\mathbf{8}, m / z 307[\mathrm{M}+\mathrm{H}]^{+}$for $\mathbf{1 0}, m / z 291[\mathrm{M}+\mathrm{H}]^{+}$for $\mathbf{1 3}$, and $m / z 293[\mathrm{M}+\mathrm{H}]^{+}$for 14.

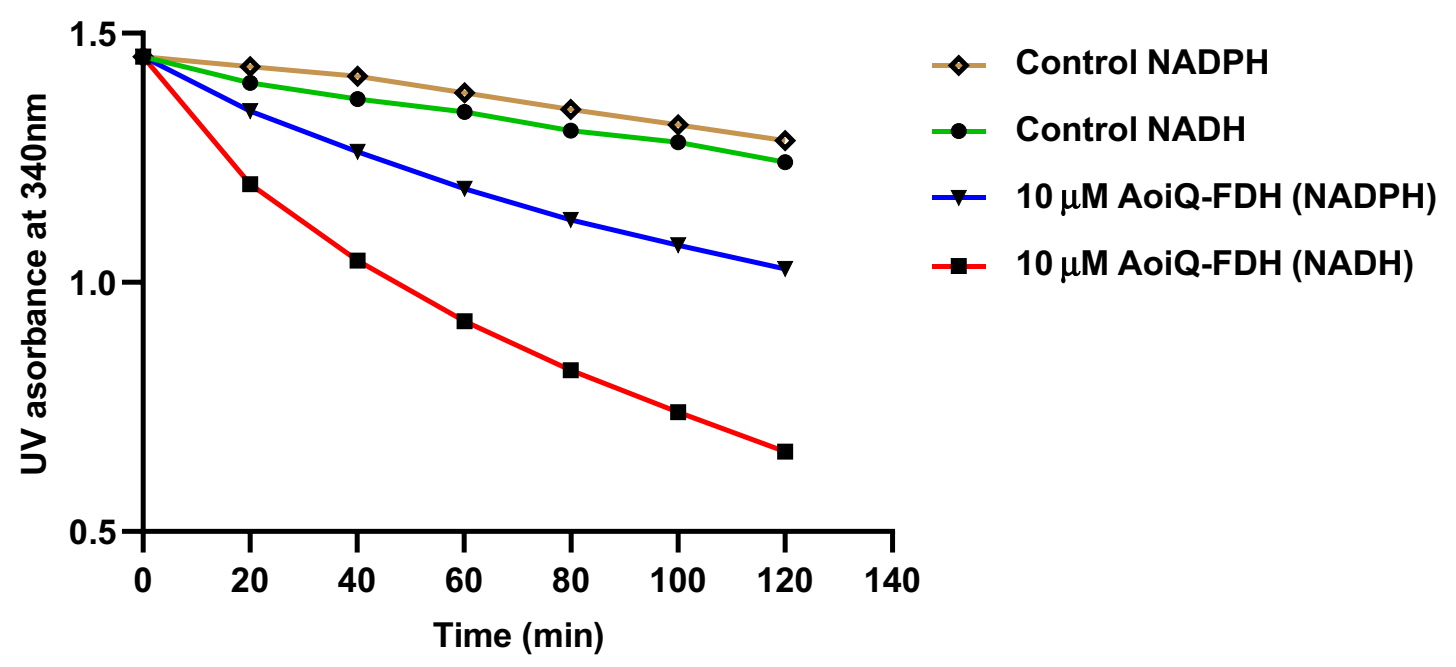

Figure S18. The flavin reductase activity of AoiQ-FDH for NADH and NADPH. Control was NADH or NADPH with boiled AoiQ-FDH. $30 \mu \mathrm{M}$ AoiQ-FDH was used in in vitro assays in the presence of $30 \mu \mathrm{M} \mathrm{FAD}, 0.2 \mathrm{mM}$ NADH or NADPH. The activity of AoiQ-FDH was determined by measuring the decrease in absorbance at $340 \mathrm{~nm}$ due to the oxidation of $\operatorname{NAD}(\mathrm{P}) \mathrm{H}\left(\Delta \varepsilon_{340}=6,220 \mathrm{M}^{-1} \cdot \mathrm{cm}^{-1}\right)$, referring to the reported method. ${ }^{[18]}$ For kinetic characterization, $10 \mu \mathrm{M}$ AoiQ-FDH was incubated with 5 to $200 \mu \mathrm{M}$ NAD(P)H and $100 \mu \mathrm{M}$ FAD in $100 \mathrm{mM} \mathrm{NaCl}$, $50 \mathrm{mM}$ phosphate buffer ( $\mathrm{pH}$ 8). The data were analyzed by GraphPad Prism 9 through nonlinear fitting of the Michaelis-Menten equation to obtain the kinetic parameters. 


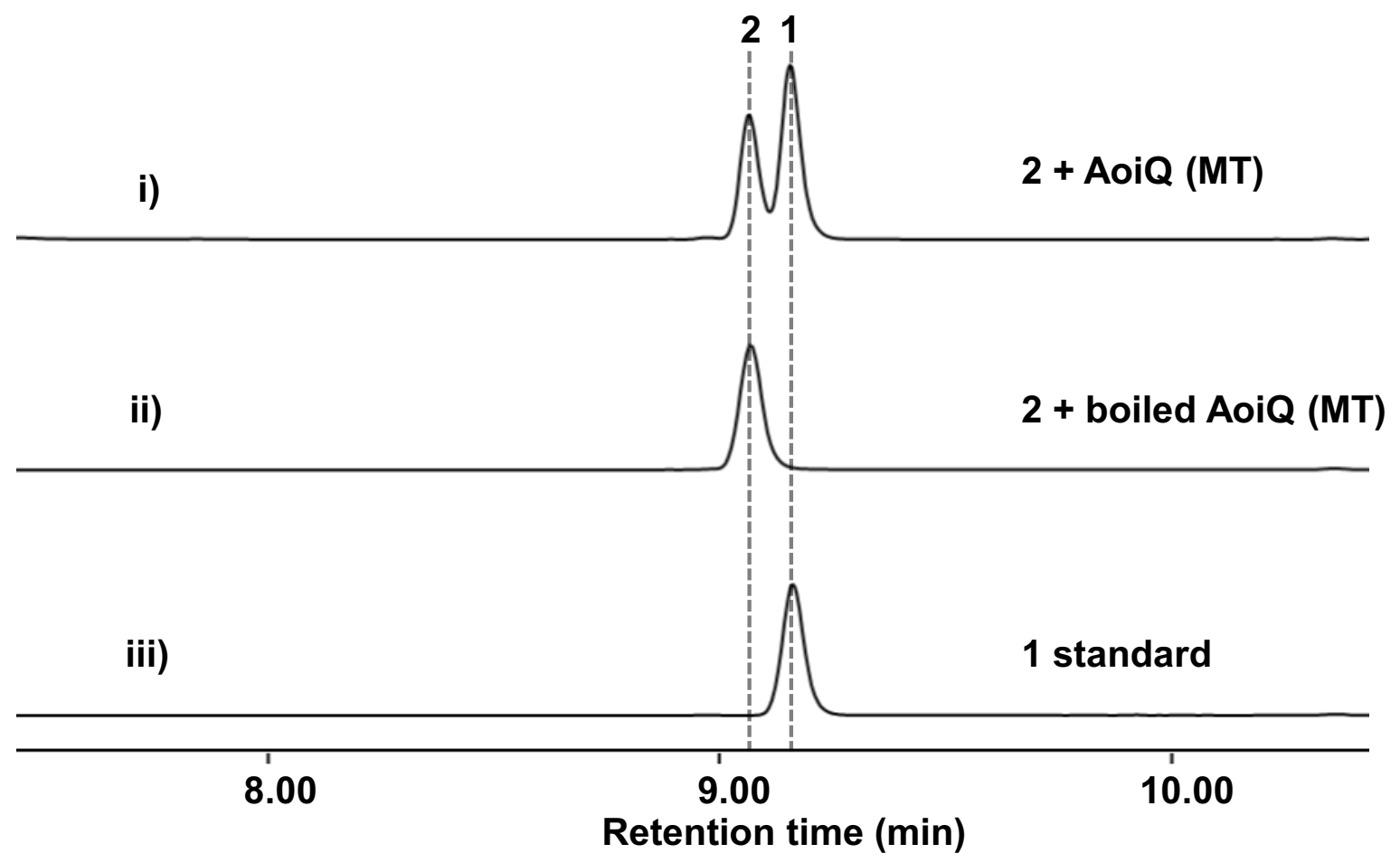

Figure S19. HPLC analysis of in vitro AoiQ (MT) enzyme requirement for dimethylation with compound 2 as substrate. The reaction mix contained $2 \mathrm{mM} \mathrm{SAM}, 500 \mu \mathrm{M}$ compound 2 , and $6 \mu \mathrm{M}$ AoiQ (MT). The reactions were incubated at $30^{\circ} \mathrm{C}$ for 0.5 hours, quenched with equal amount of $\mathrm{MeCN}$ to terminate the reaction. 


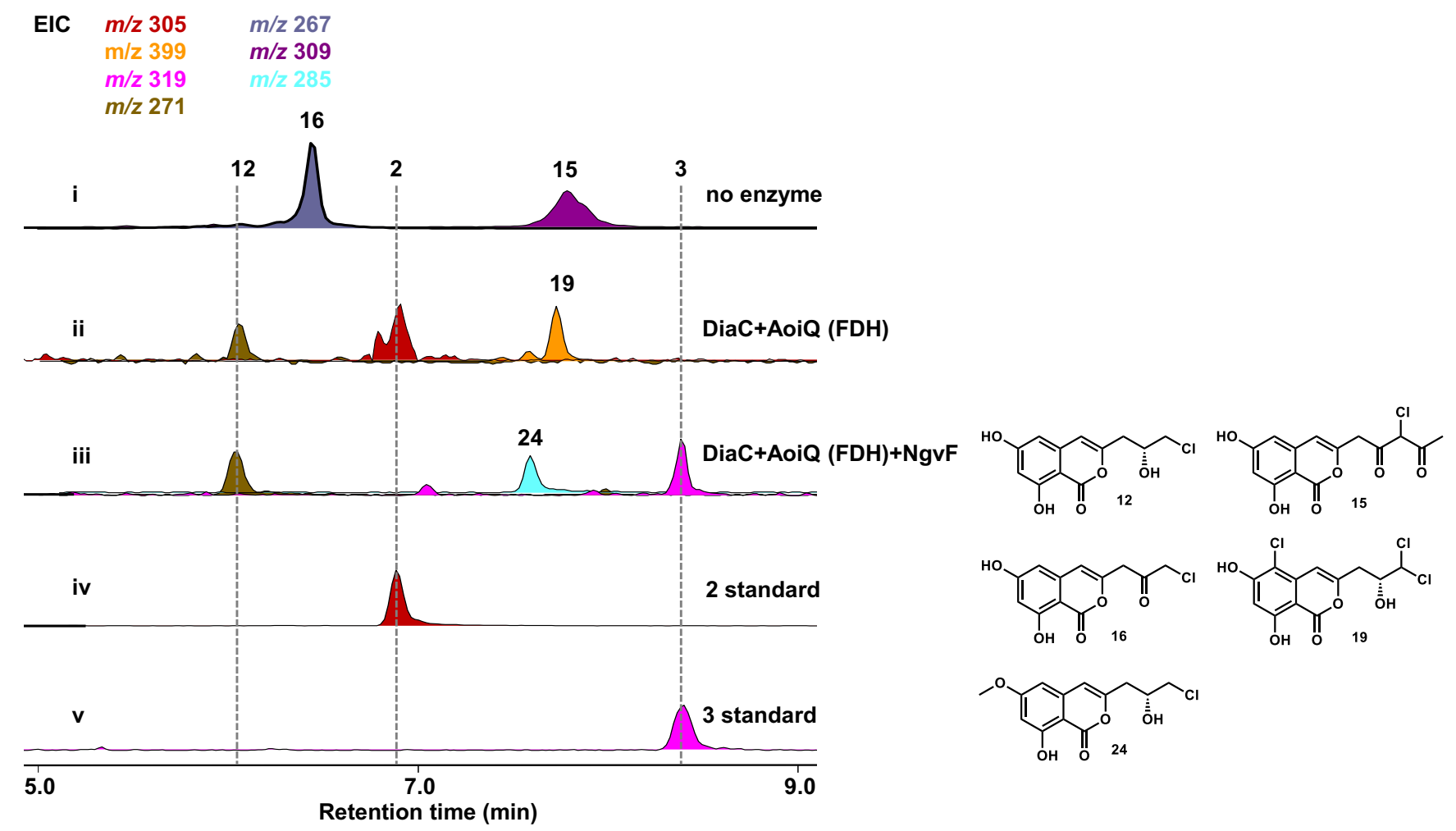

Figure S20. LC/MS analysis of enzymatic reaction of AoiQ (FDH) domain with synthetic compound $\mathbf{1 5}$ as substrate. The reaction mix contained $5 \mu \mathrm{M} \mathrm{DiaC}, 50 \mu \mathrm{M}$ FAD, $10 \mathrm{mM}$ NAPH, $25 \mathrm{mM} \mathrm{NaCl}, 50 \mathrm{mM}$ phosphate buffer (PH 6.7), and/or $6 \mu \mathrm{M} \mathrm{NgvF}$ with $1 \mathrm{mM}$ SAM, $500 \mu \mathrm{M}$ compound 15 and $150 \mu \mathrm{M}$ AoiQ (FDH). The reactions were incubated at $30{ }^{\circ} \mathrm{C}$ for 3 hours, quenched with equal amount of $\mathrm{MeCN}$ to terminate reaction. The extracted ion chromatograms (EICs) were extracted at $m / z 271[\mathrm{M}+\mathrm{H}]^{+}$for 12, $m / z 267[\mathrm{M}-\mathrm{H}]^{-}$for 16, $\mathrm{m} / z 399[\mathrm{M}+\mathrm{H}]^{+}$for 19, $m / z 309[\mathrm{M}-\mathrm{H}]^{-}$for 15, $m / z 319[\mathrm{M}+\mathrm{H}]^{+}$for $\mathbf{3}, \mathrm{m} / z 285[\mathrm{M}+\mathrm{H}]^{+}$for $\mathbf{2 4}, m / z 305[\mathrm{M}+\mathrm{H}]^{+}$for 2. 


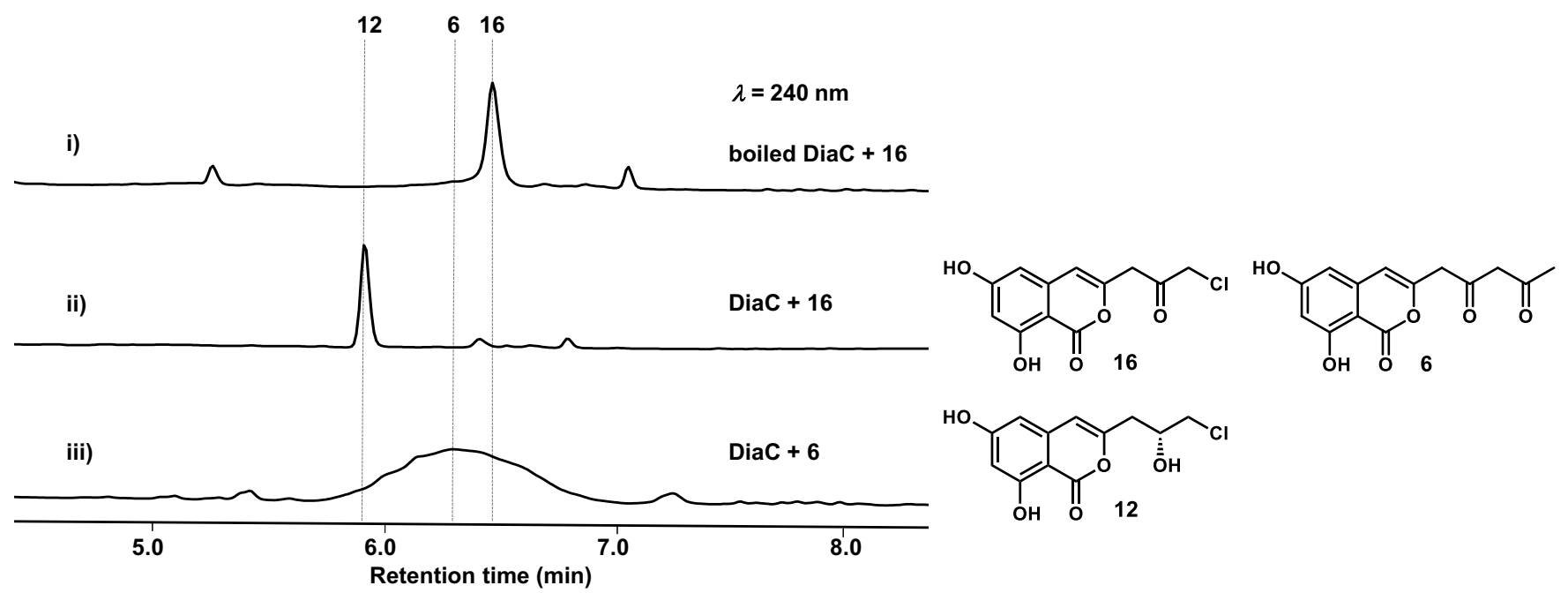

Figure S21. LC/MS analysis of enzymatic reaction of $\mathrm{DiaC}$ with $\mathbf{6}$ or $\mathbf{1 6}$ as substrate. The reaction mix contained $6 \mu \mathrm{M}$ DiaC, $5 \mathrm{mM}$ NADH, $50 \mathrm{mM}$ phosphate buffer (PH 6.7, and $500 \mu \mathrm{M}$ substrate. The reactions were incubated at $30{ }^{\circ} \mathrm{C}$ for $15 \mathrm{~min}$, quenched with equal amount of $\mathrm{MeCN}$ to terminate reaction. 
A

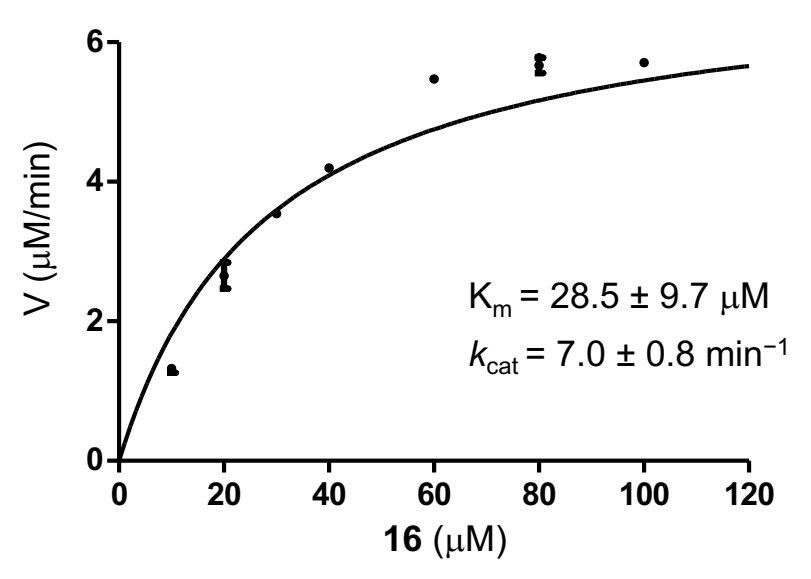

B

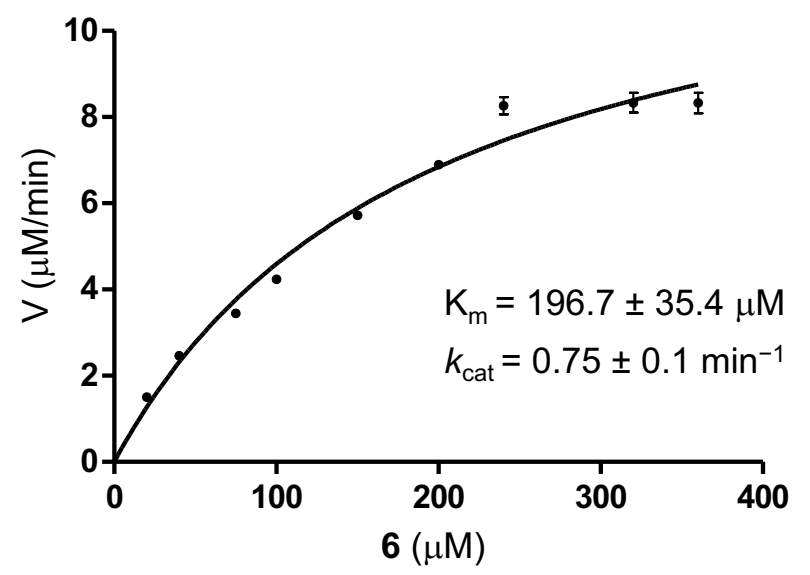

Figure S22. Kinetic parameters of DiaC-catalyzed the conversion of $\mathbf{1 6}$ to 12 (A) and 6 to 8 (B), respectively. Concentrations of DiaC were $15 \mu \mathrm{M}$ for 6 and $1 \mu \mathrm{M}$ for 16. The assays were performed with $1 \mu \mathrm{M}$ DiaC and 10-100 $\mu \mathrm{M} 16$ in phosphate buffer $(50 \mathrm{mM}, \mathrm{pH} 6.7), 30^{\circ} \mathrm{C}$ for $15 \mathrm{~min}$. Concentrations of DiaC were $15 \mu \mathrm{M}$ for 20-360 $\mu \mathrm{M} 6$ in phosphate buffer $(50 \mathrm{mM}, \mathrm{pH} 6.7), 30^{\circ} \mathrm{C}$ for $30 \mathrm{~min}$. 

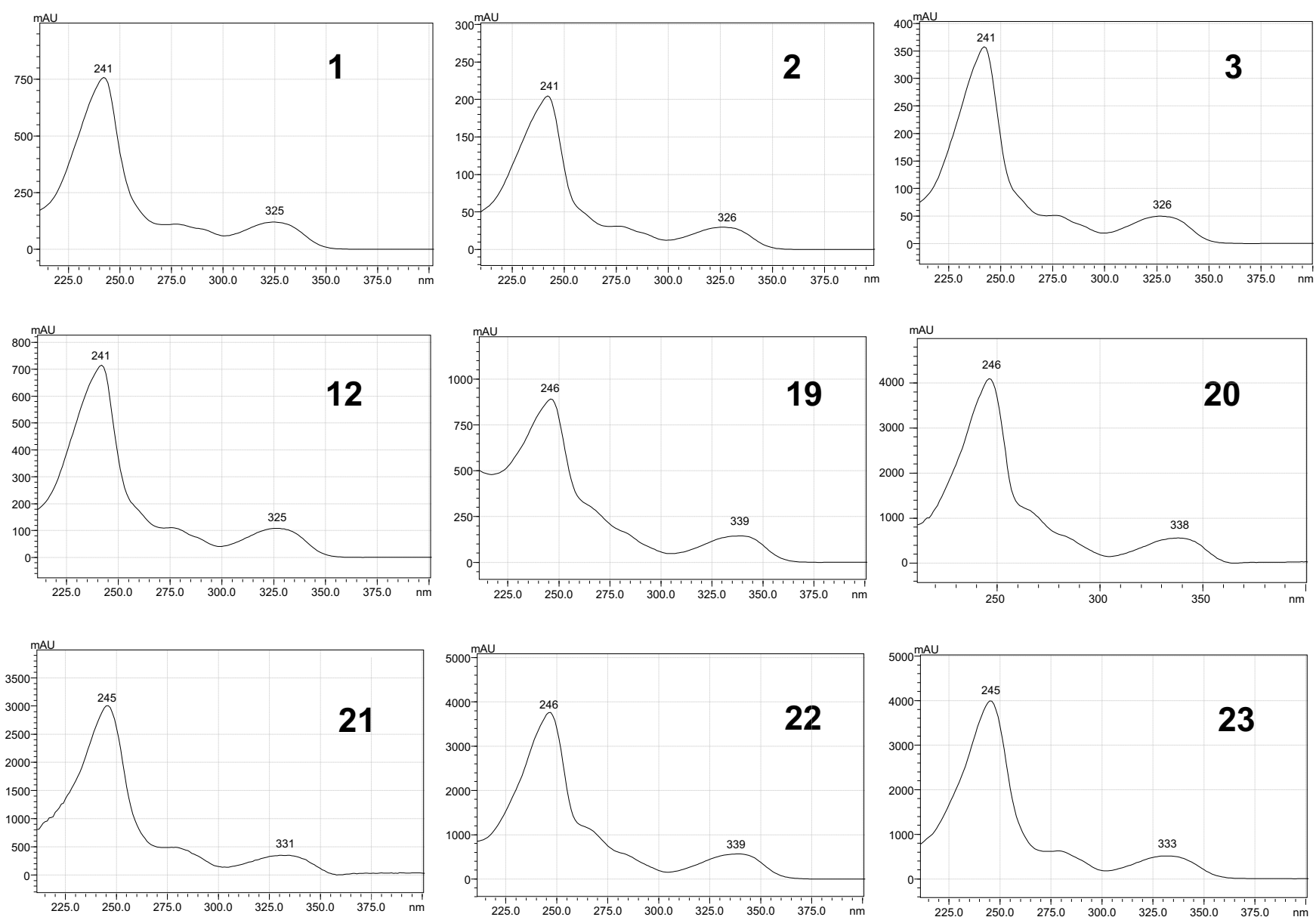

Figure S23. UV profiles of compounds of 1, 2, 3, 12, 19, 20, 21, 22, and 23. 


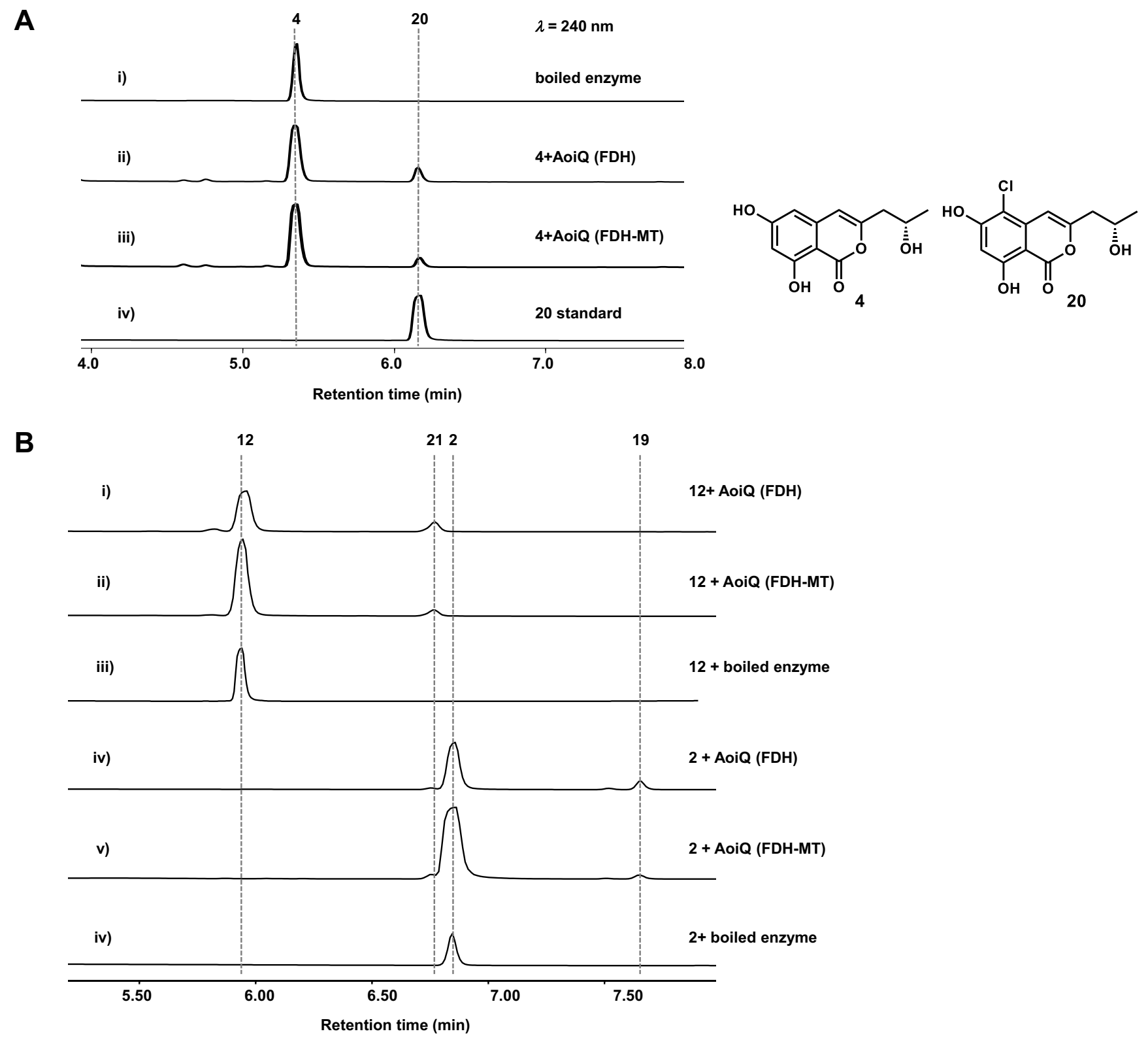

Figure S24. LC/MS analysis of enzymatic reaction of AoiQ (FDH) domain or AoiQ (FDH-MT) with A) substrate 4; B) substrate 2 or 12. The reaction mix contained $150 \mu \mathrm{M}$ AoiQ (FDH) or AoiQ (FDH-MT), $50 \mu \mathrm{M}$ FAD, $10 \mathrm{mM} \mathrm{NADH}, 25 \mathrm{mM} \mathrm{NaCl}, 50 \mathrm{mM}$ phosphate buffer (PH 6.7), and $500 \mu \mathrm{M}$ substrate. The reactions were incubated at $30{ }^{\circ} \mathrm{C}$ for 3 hours, quenched with equal amount of $\mathrm{MeCN}$ to terminate reaction. 


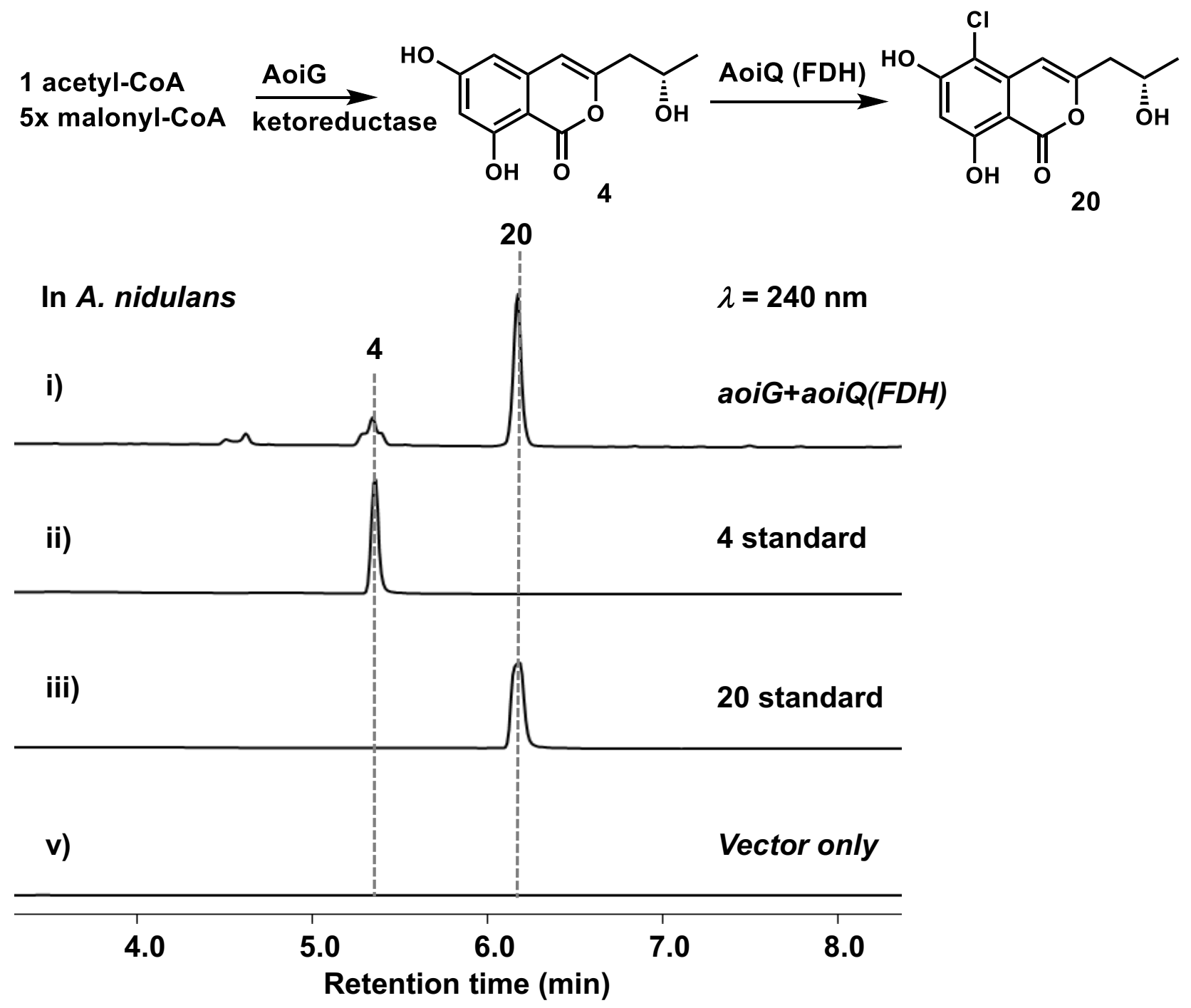

Figure S25. LC/MS analysis of extracts from A. nidulans expressing AoiG and AoiQ-FDH (trace i). 


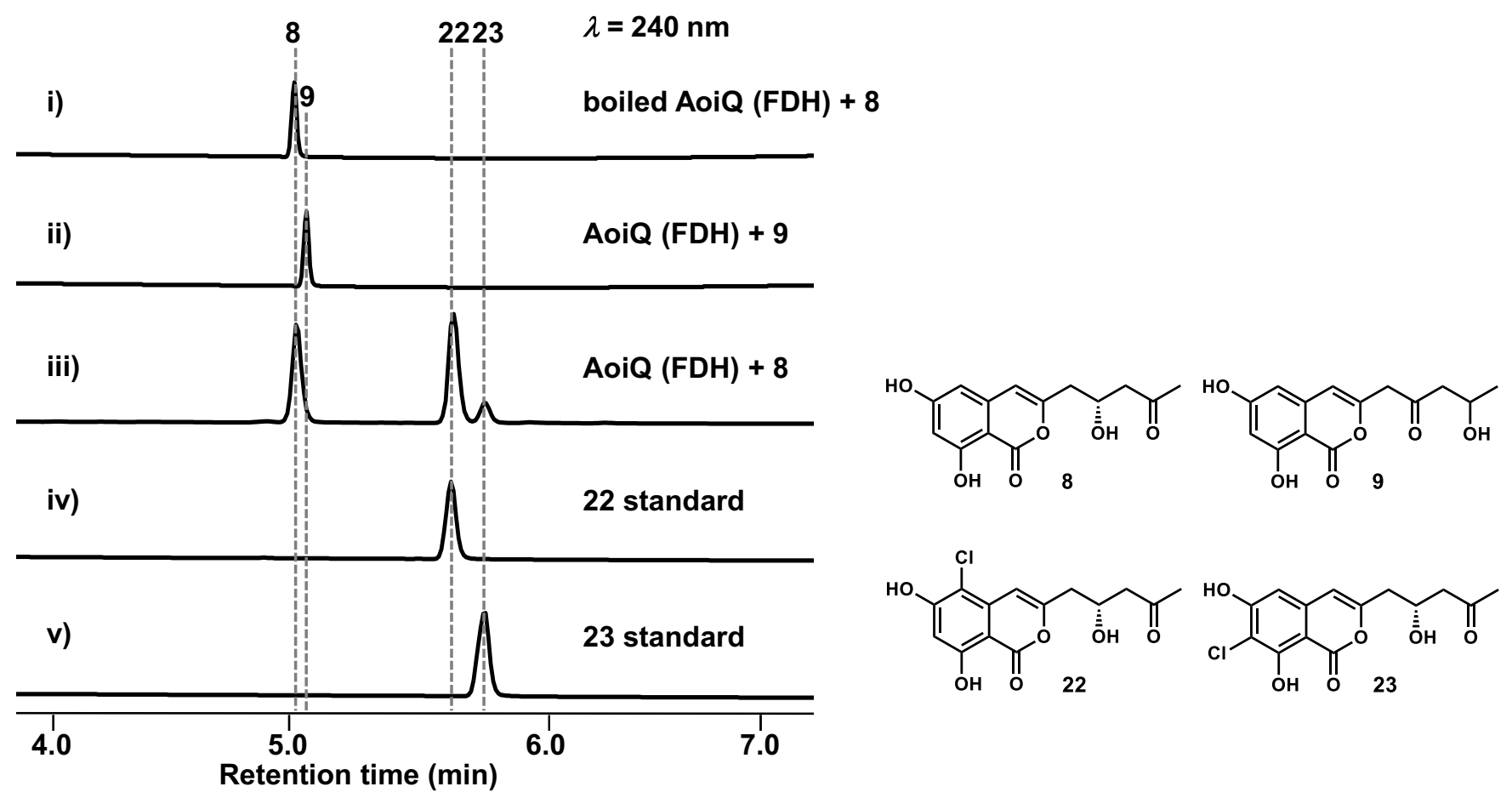

Figure S26. LC/MS analysis of enzymatic reaction of AoiQ (FDH) domain with $\mathbf{8}$ and $\mathbf{9}$ as substrate. The reaction mix contained $150 \mu \mathrm{M}$ AoiQ (FDH), $50 \mu \mathrm{M}$ FAD, $10 \mathrm{mM} \mathrm{NADH}, 25 \mathrm{mM} \mathrm{NaCl}, 50 \mathrm{mM}$ phosphate buffer (PH 6.7), and $500 \mu \mathrm{M}$ compound 8 or 9 . The reactions were incubated at $30^{\circ} \mathrm{C}$ for 3 hours, quenched with equal amount of $\mathrm{MeCN}$ to terminate the reaction. 


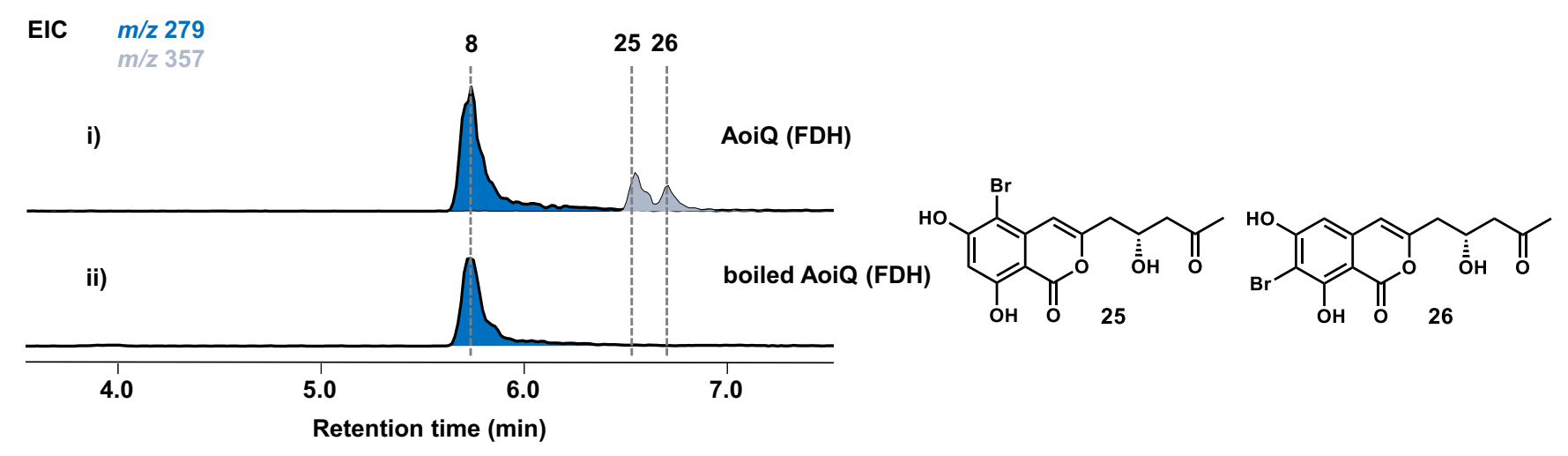

Figure S27. LC/MS analysis of enzymatic reaction of AoiQ (FDH) domain using bromo as halogen donor. The reaction mix contained $150 \mu \mathrm{M}$ AoiQ (FDH), $50 \mu \mathrm{M}$ FAD, $10 \mathrm{mM} \mathrm{NADH}, 50 \mathrm{mM} \mathrm{NaBr}, 50 \mathrm{mM}$ phosphate buffer (PH 6.7), and $500 \mu \mathrm{M}$ compound 8. The extracted ion chromatograms (EICs) were extracted at $m / z 279$ [M $+\mathrm{H}]^{+}$for $\mathbf{8}, m / z 357[\mathrm{M}+\mathrm{H}]^{+}$for $\mathbf{2 5}$ and $\mathbf{2 6}$. 


\section{NMR spectra}

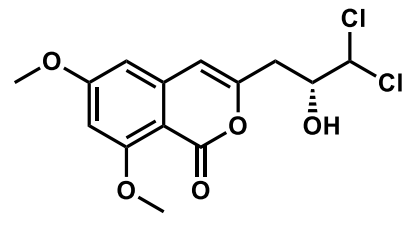

8-methyldichlorodiaporthin (1)
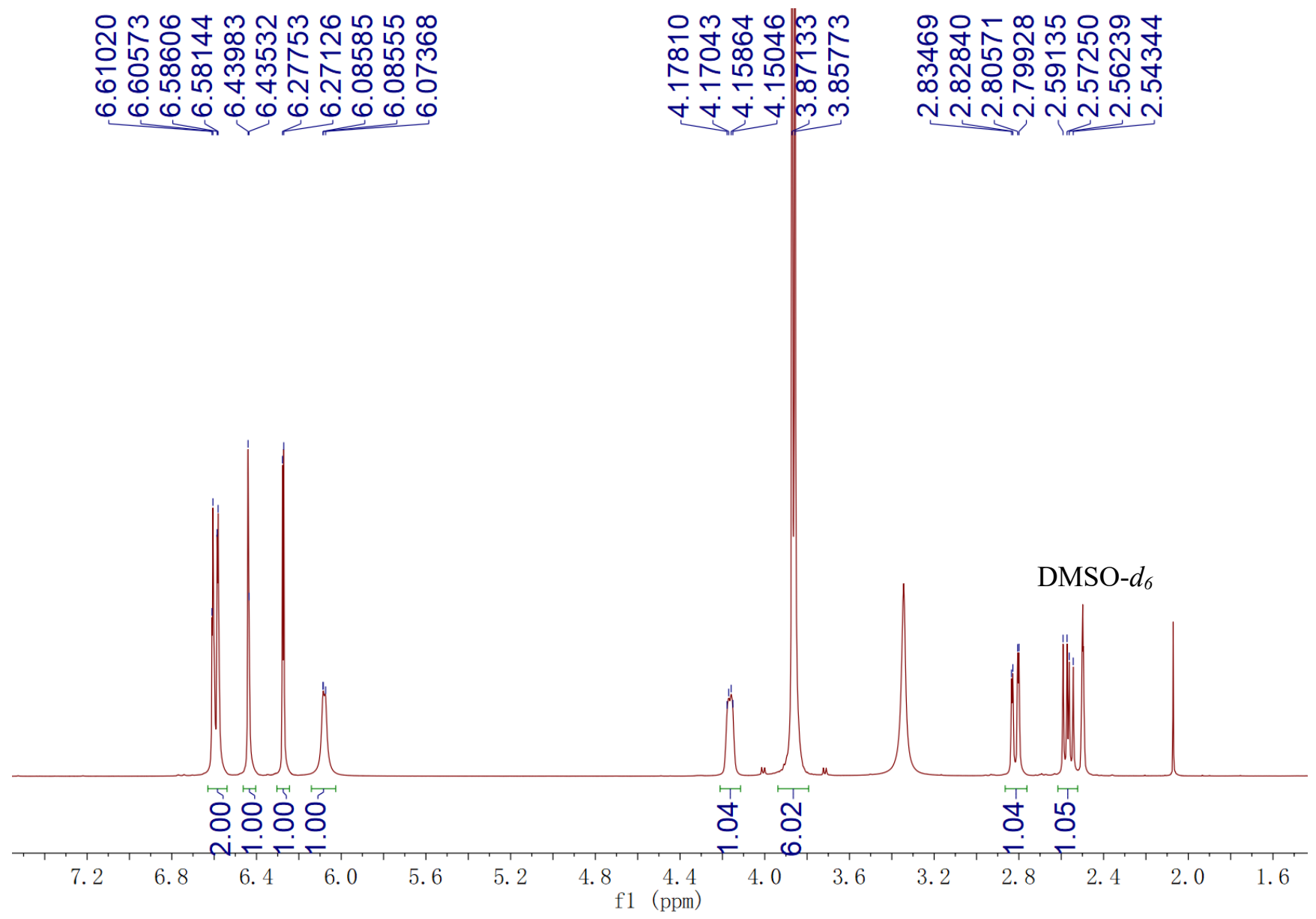

Figure S28. ${ }^{1} \mathrm{H}$ NMR spectrum of compound 1 in DMSO- $d_{6}$. 


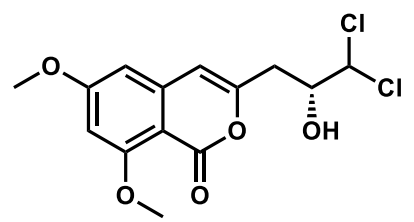

8-methyldichlorodiaporthin (1)

\section{DMSO- $d_{6}$}

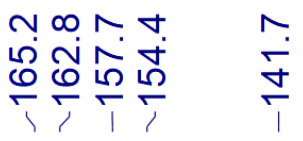

ำ잉

ํํㅇํㅇํำ

$-N$

$\hat{N}$

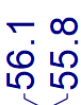

சே.

厂

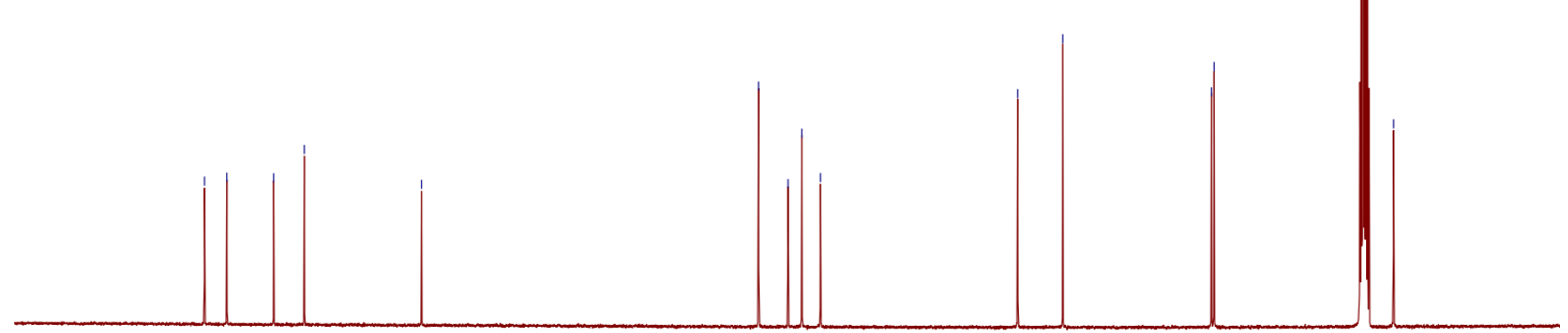

$\begin{array}{llllllllllllllll}180 & 170 & 160 & 150 & 140 & 130 & 120 & 110 \begin{array}{c}100 \\ \mathrm{f} 1(\mathrm{ppm})\end{array} & 90 & 80 & 70 & 60 & 50 & 40 & 30 & 20\end{array}$

Figure S29. ${ }^{13} \mathrm{C}$ NMR spectrum of compound 1 in DMSO- $d_{6}$. 


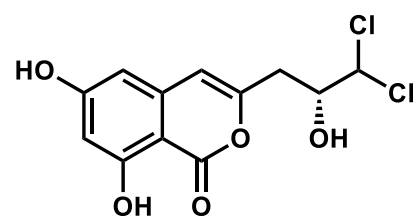

demethyldichlorodiaporthin (2)
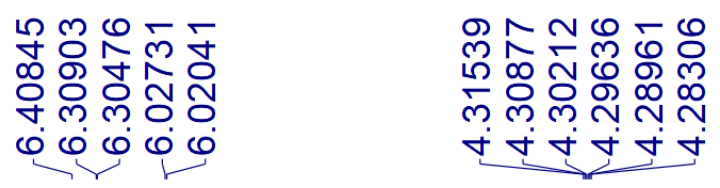

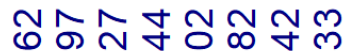

œ

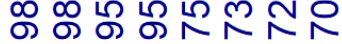

๑

กับกับกั่

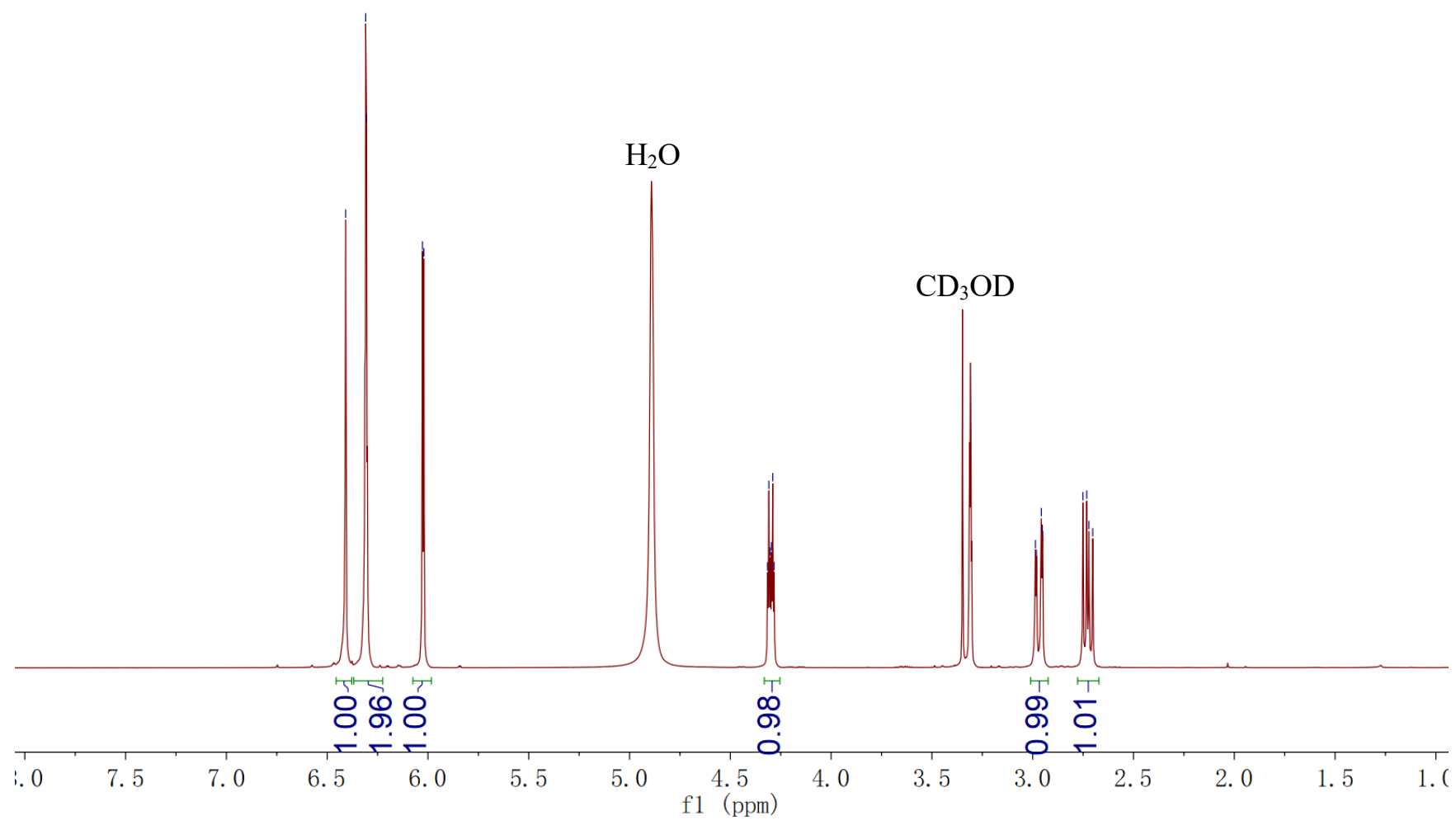

Figure S30. ${ }^{1} \mathrm{H}$ NMR $(500 \mathrm{MHz})$ spectrum of compound 2 in $\mathrm{CD}_{3} \mathrm{OD}$. 


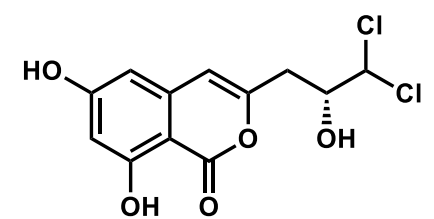

demethyldichlorodiaporthin (2)

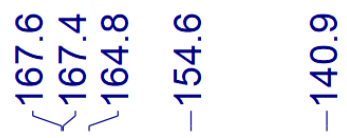

N $\infty$

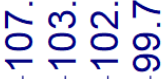

No

人N

N

n.

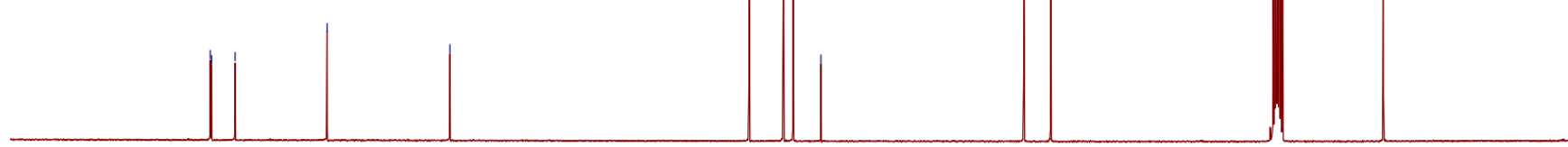

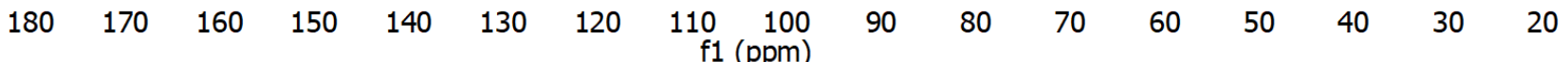

Figure S31. ${ }^{13} \mathrm{C}(125 \mathrm{MHz}) \mathrm{NMR}$ spectrum of compound 2 in $\mathrm{CD}_{3} \mathrm{OD}$. 


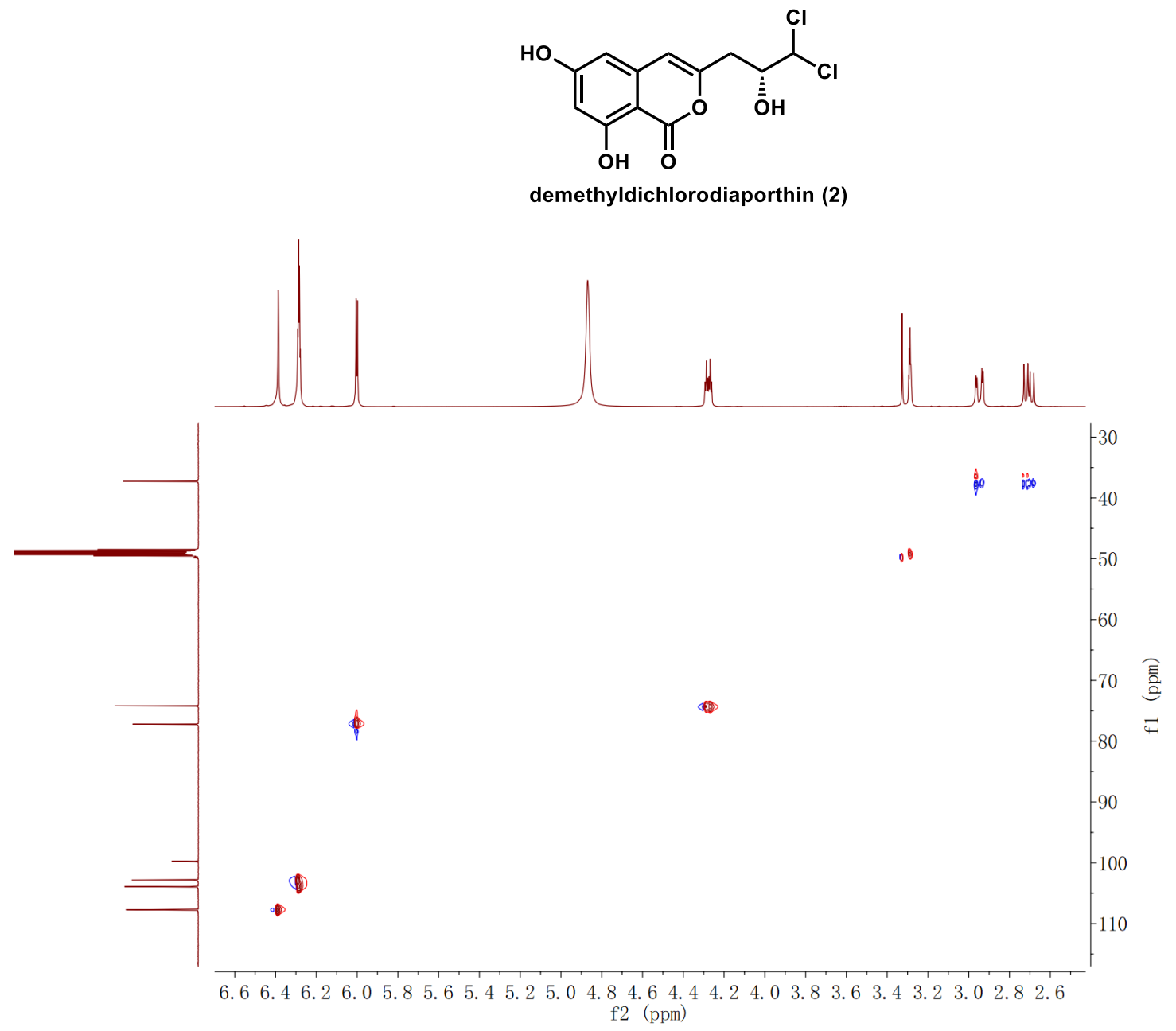

Figure 32. $\mathrm{HSQC}$ spectrum of compound 2 in $\mathrm{CD}_{3} \mathrm{OD}$. 


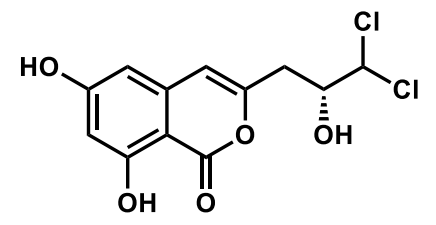

demethyldichlorodiaporthin (2)

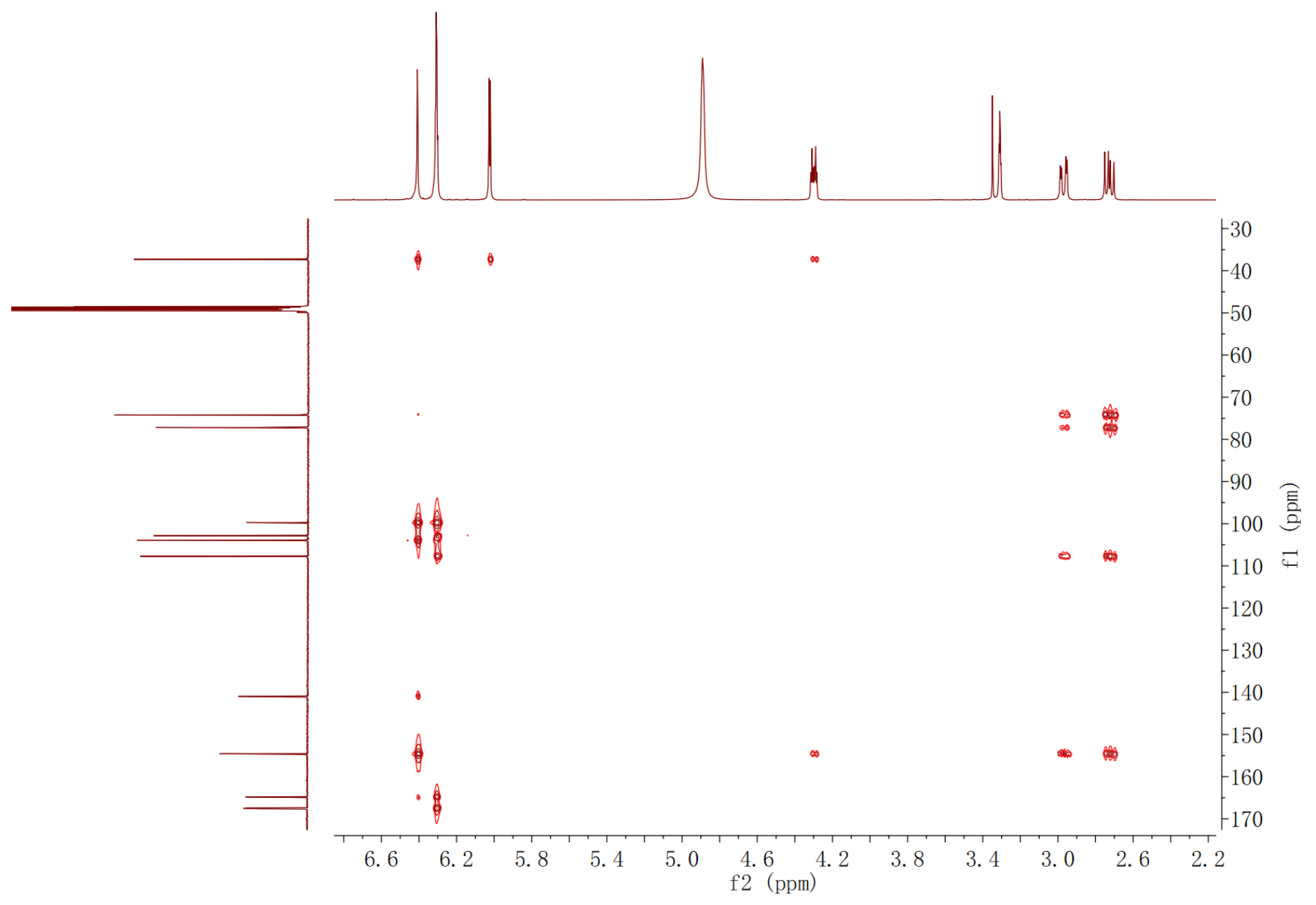

Figure S33. HMBC spectrum of compound 2 in $\mathrm{CD}_{3} \mathrm{OD}$. 


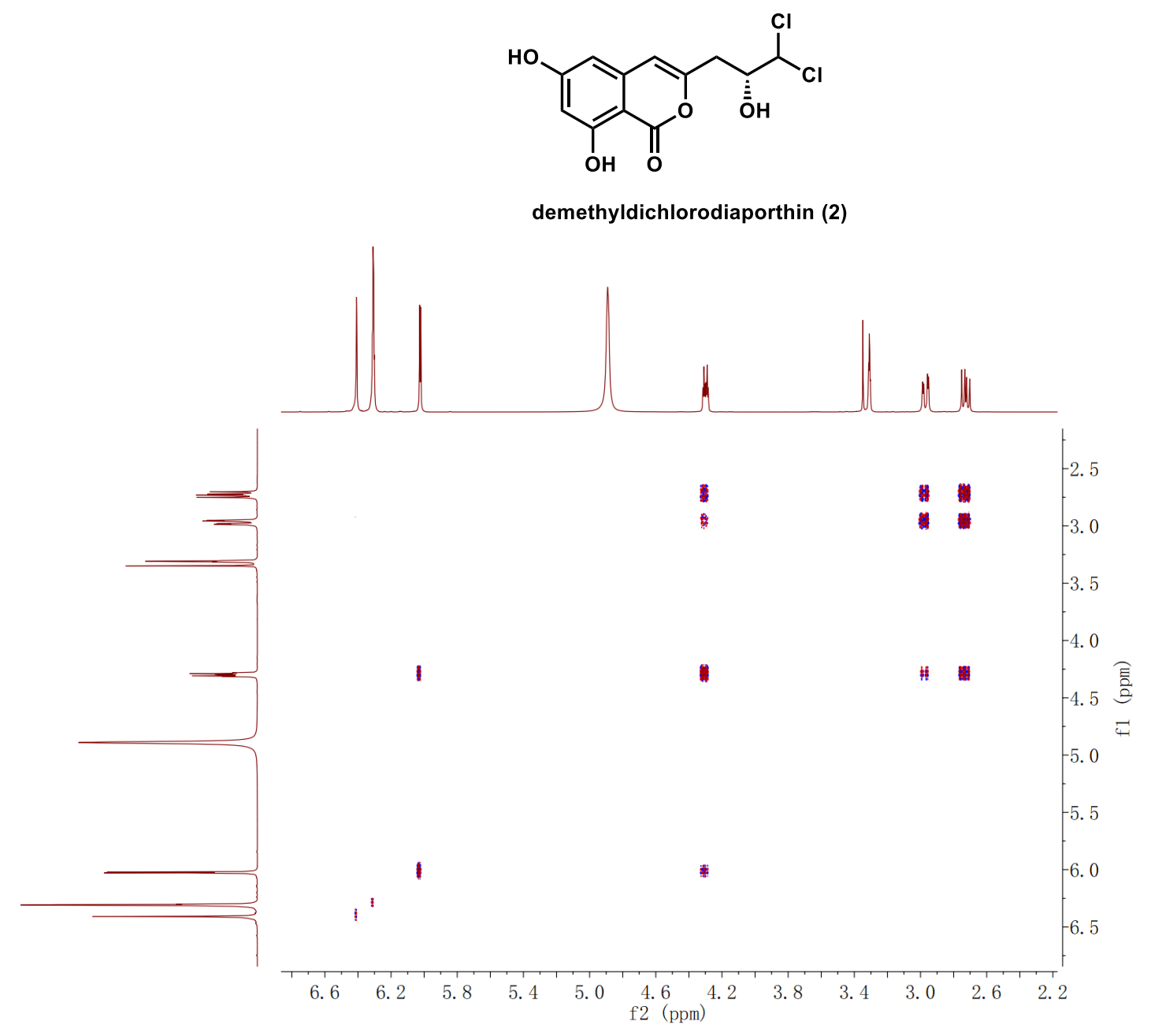

Figure S34. The ${ }^{1} \mathrm{H}-{ }^{1} \mathrm{H}$ COSY spectrum of compound 2 in $\mathrm{CD}_{3} \mathrm{OD}$. 


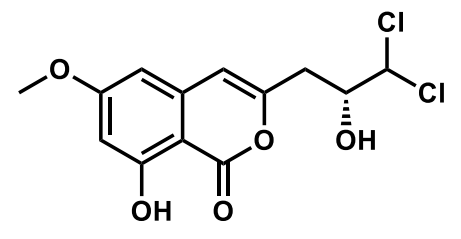

dichlorodiaporthin (3)

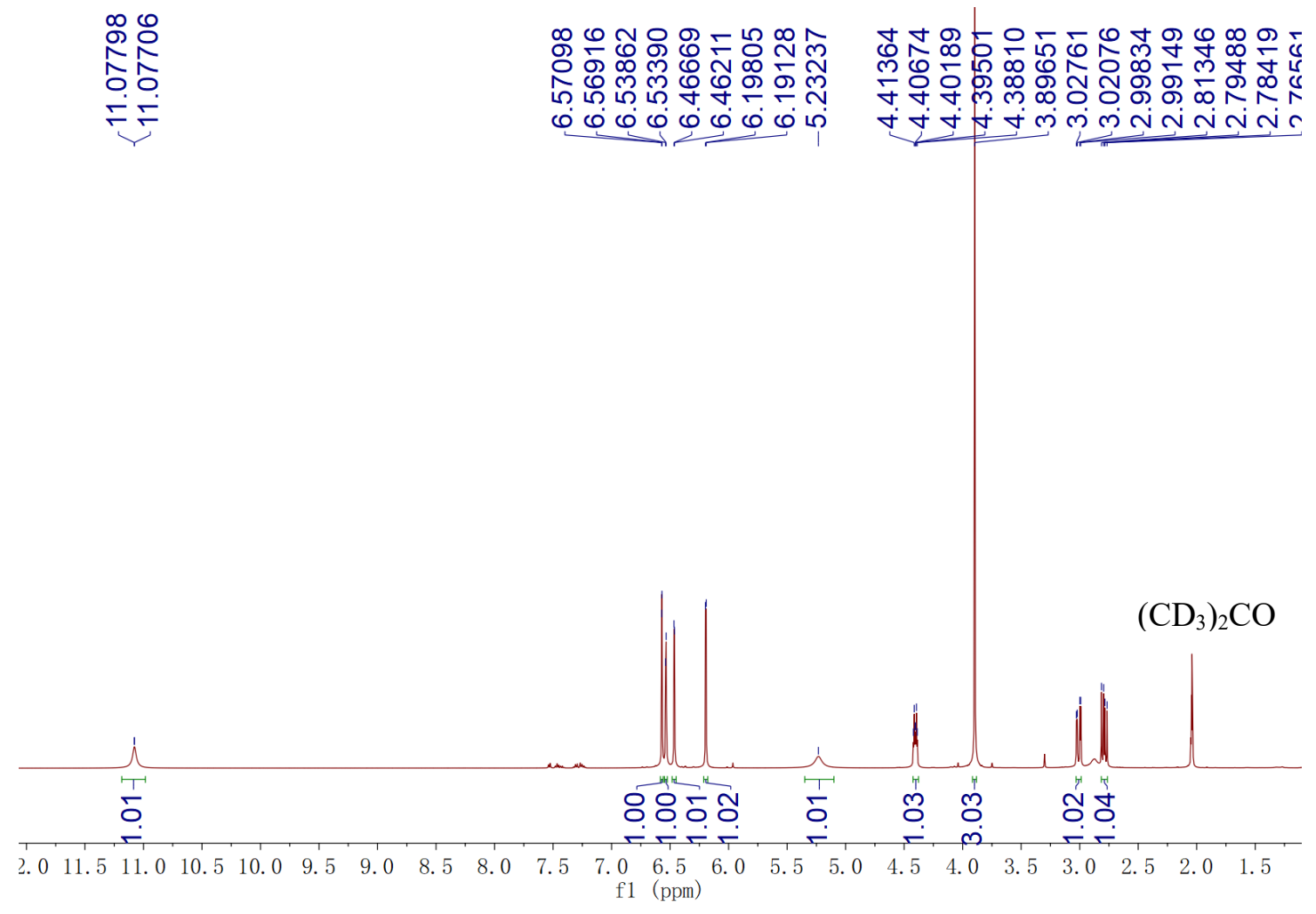

Figure S35. The ${ }^{1} \mathrm{H}$ NMR spectrum of compound $\mathbf{3}$ in $\left(\mathrm{CD}_{3}\right)_{2} \mathrm{CO}$. 


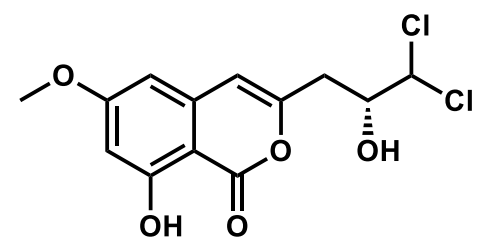

dichlorodiaporthin (3)

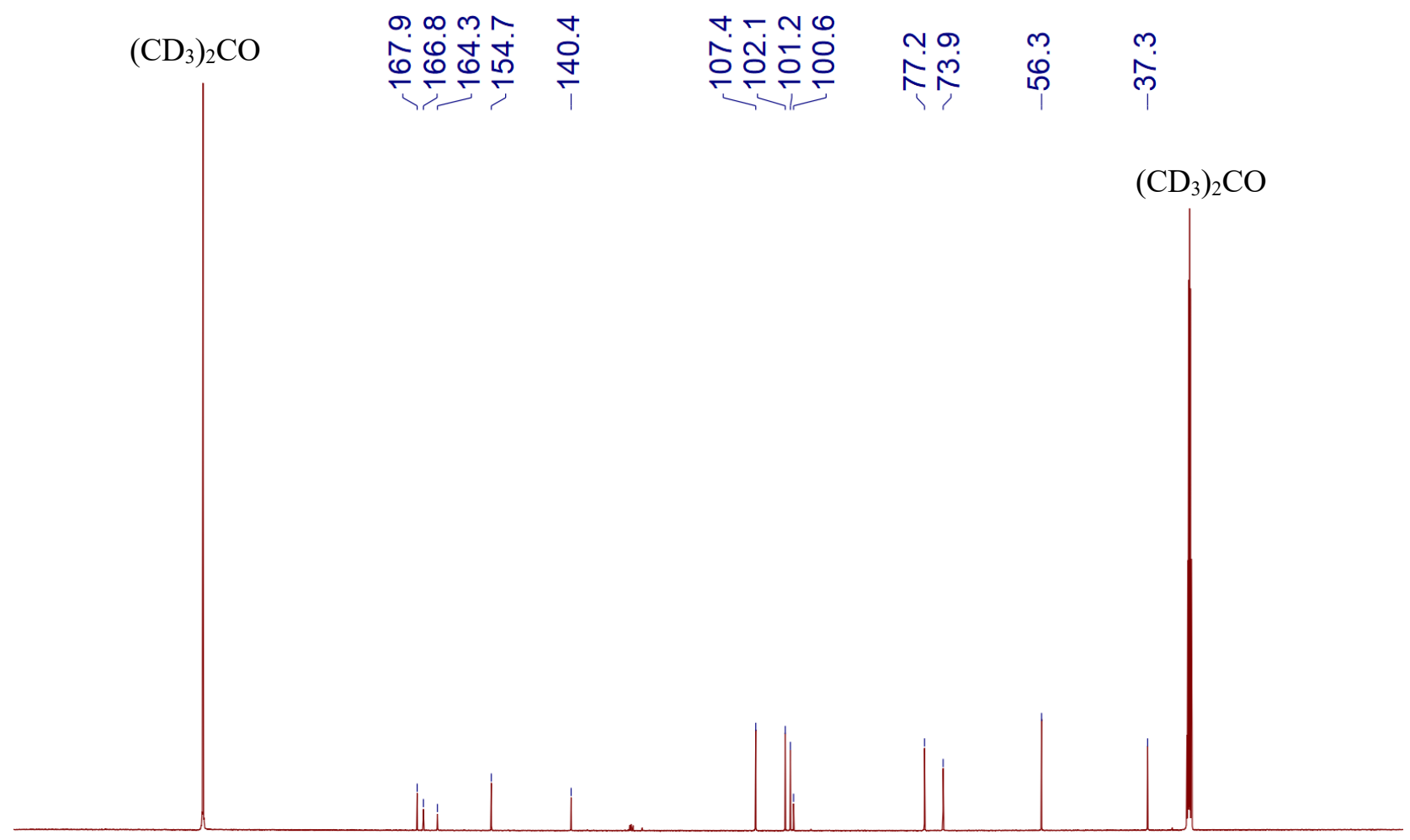

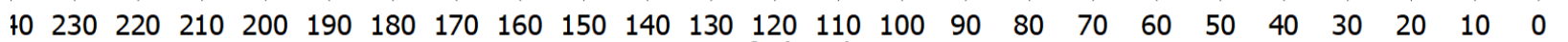
f1 (ppm)

Figure S36. The ${ }^{13} \mathrm{C}$ NMR spectrum of compound 3 in $\left(\mathrm{CD}_{3}\right)_{2} \mathrm{CO}$. 


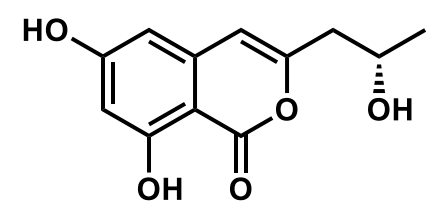

orthosporin (4)
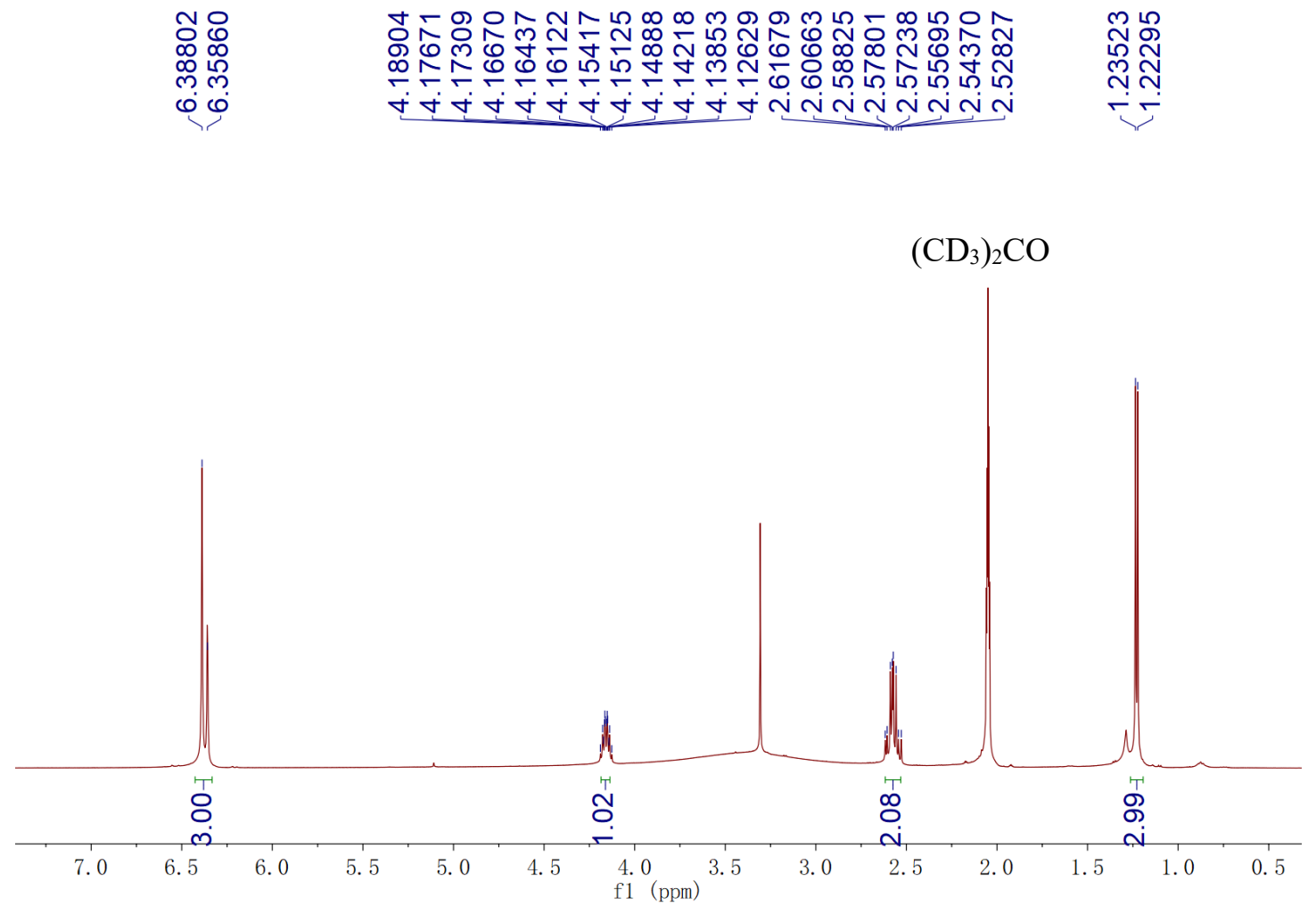

Figure S37. The ${ }^{1} \mathrm{H}$ NMR spectrum of compound 4 in $\left(\mathrm{CD}_{3}\right)_{2} \mathrm{CO}$. 


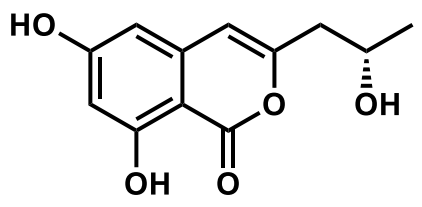

orthosporin (4)

$\left(\mathrm{CD}_{3}\right)_{2} \mathrm{CO}$

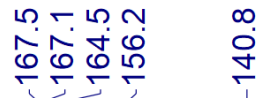

$\forall N$ r

ํํํํํㅇํㅇ

๑)

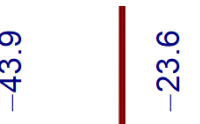

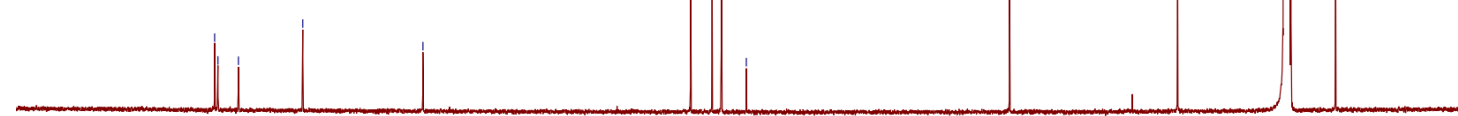

$\begin{array}{lllllllllllllllllll}190 & 180 & 170 & 160 & 150 & 140 & 130 & 120 & 110 & \begin{array}{c}100 \\ \mathrm{f} 1(\mathrm{ppm})\end{array} & 90 & 80 & 70 & 60 & 50 & 40 & 30 & 20 & 10\end{array}$

Figure S38. The ${ }^{13} \mathrm{C}$ NMR spectrum of compound 4 in $\left(\mathrm{CD}_{3}\right)_{2} \mathrm{CO}$. 


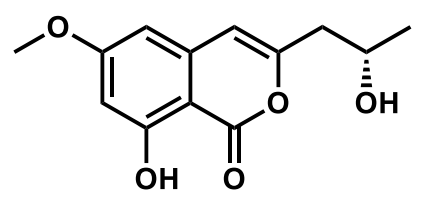

diaporthin (5)
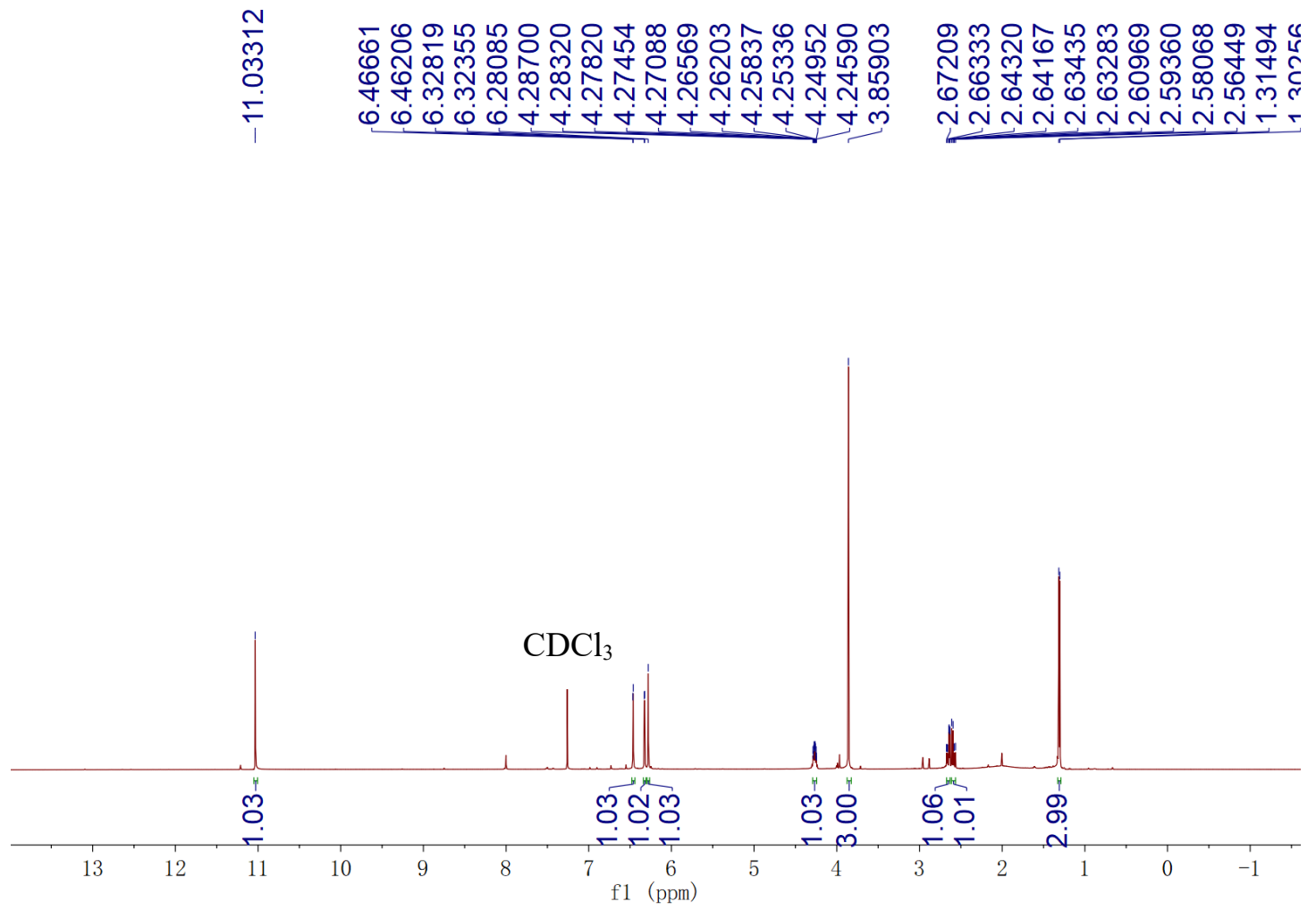

Figure S39. The ${ }^{1} \mathrm{H}$ NMR spectrum of compound 5 in $\mathrm{CDCl}_{3}$. 


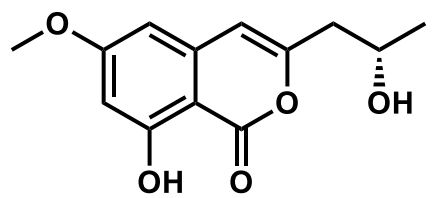

diaporthin (5)

の 000 ต ต ז

$\frac{n}{n}$

n $-N$ -

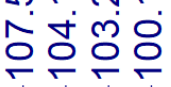

กั

र्र

n.

\section{$\mathrm{CDCl}_{3}$}

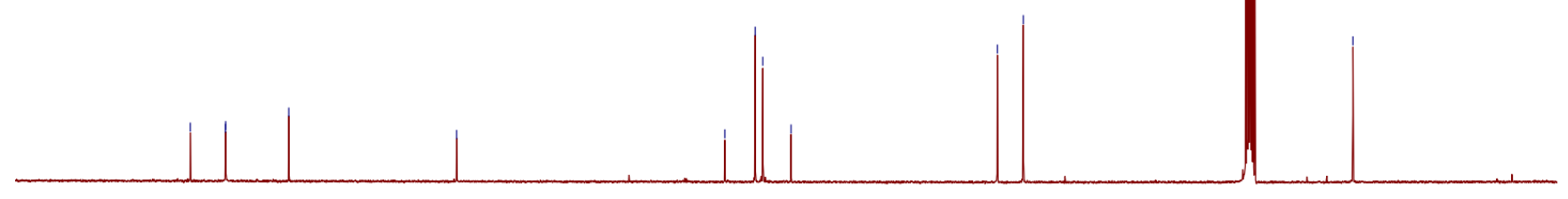

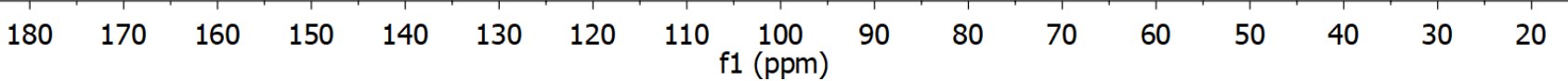

Figure S40. The ${ }^{13} \mathrm{C}$ NMR spectrum of compound 5 in $\mathrm{CDCl}_{3}$. 


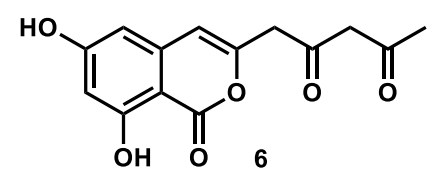

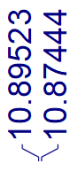

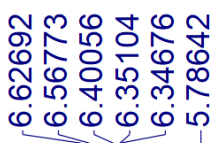

낭유은

织告

लंm

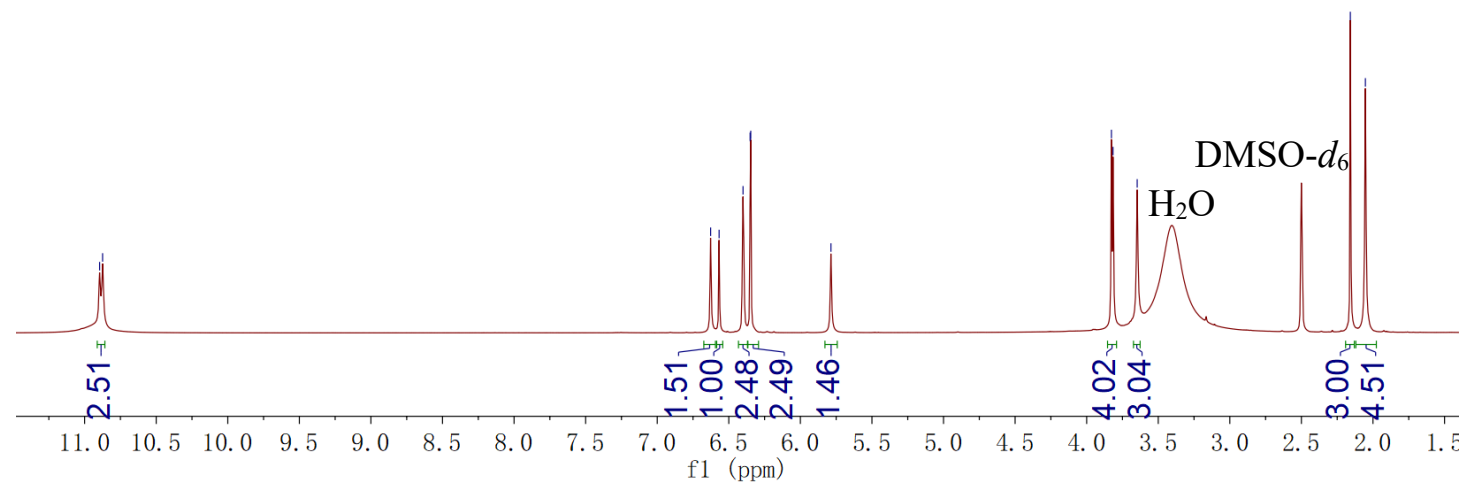

Figure S41. The ${ }^{1} \mathrm{H}$ NMR spectrum of compound 6 in DMSO- $d_{6}$. 


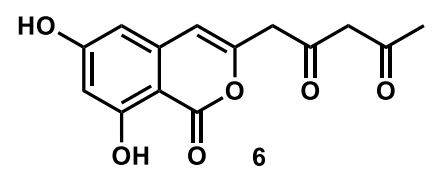

\section{DMSO- $d_{6}$}

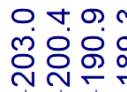

$\infty \sim T \leftarrow$

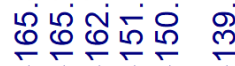

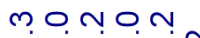

드응ํㅇํㅇํำ

60. ถ๊ำ

$\infty \infty$

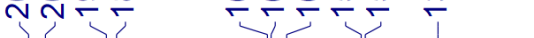

क्रें के

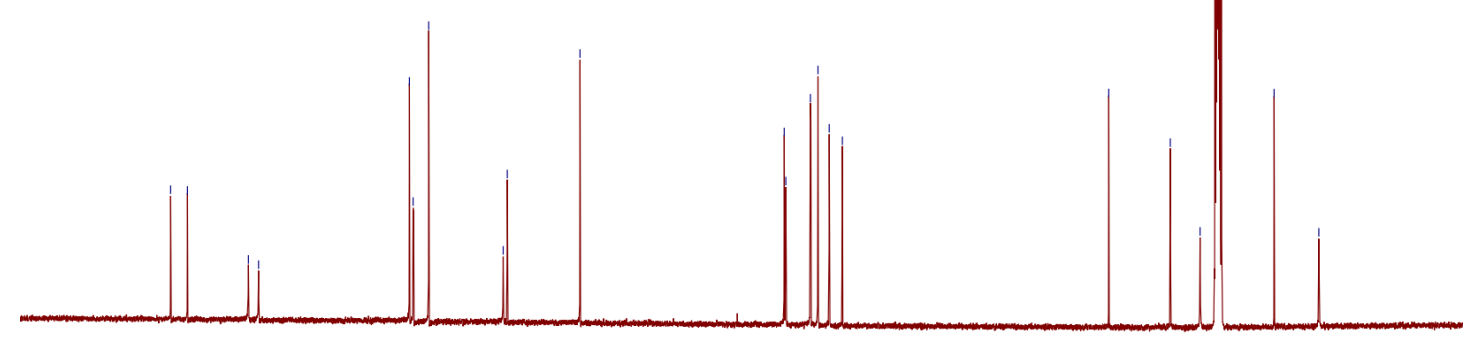

$\begin{array}{llllllllllllllllllllll}220 & 210 & 200 & 190 & 180 & 170 & 160 & 150 & 140 & 130 & 120 & 110 & 100 & 90 & 80 & 70 & 60 & 50 & 40 & 30 & 20 & 10\end{array}$

Figure S42. The ${ }^{13} \mathrm{C}$ NMR spectrum of compound 6 in DMSO- $d_{6}$. 

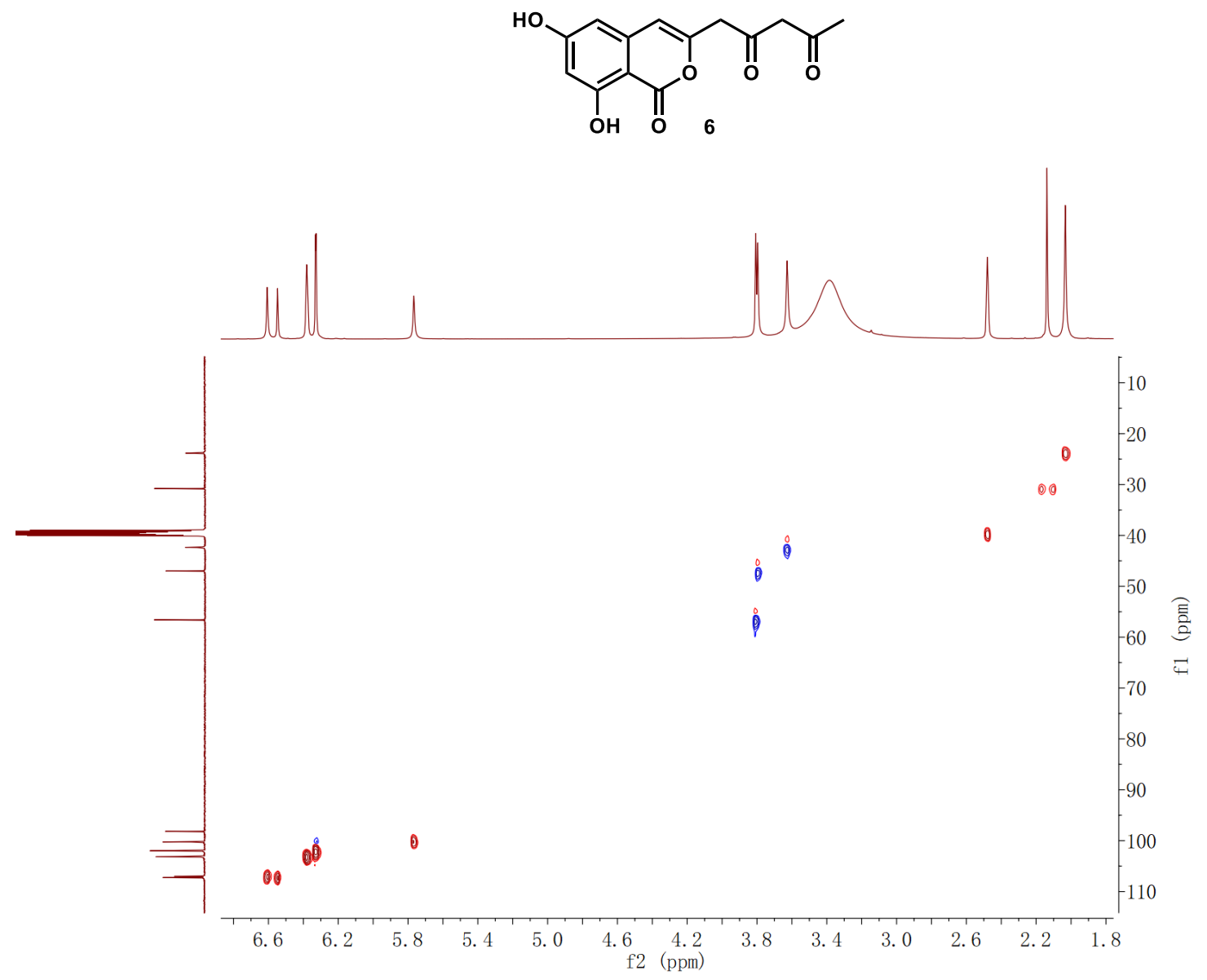

Figure S43. The HSQC spectrum of compound 6 in DMSO- $d_{6}$. 

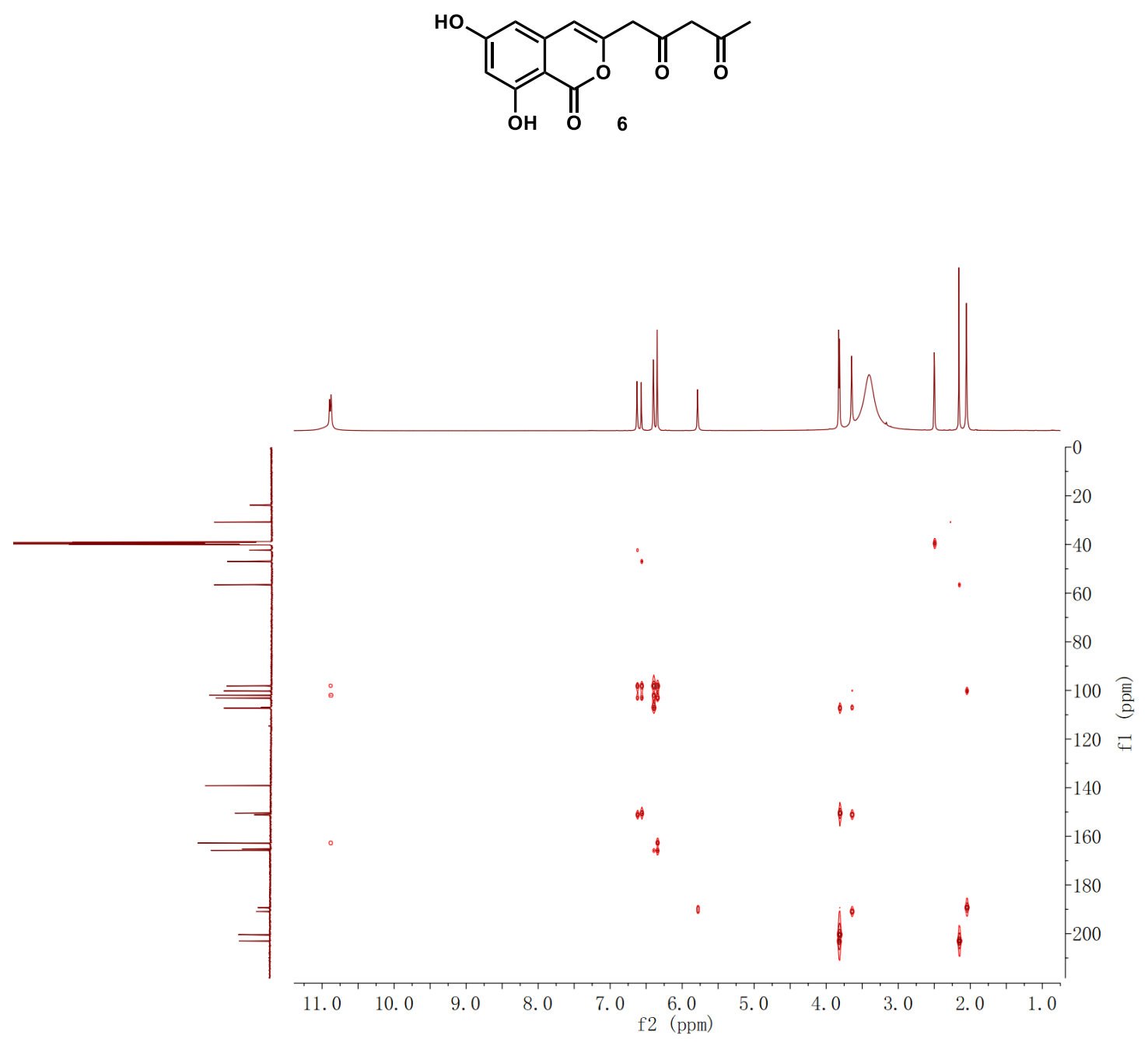

Figure S44. The HMBC spectrum of compound 6 in DMSO- $d_{6}$. 

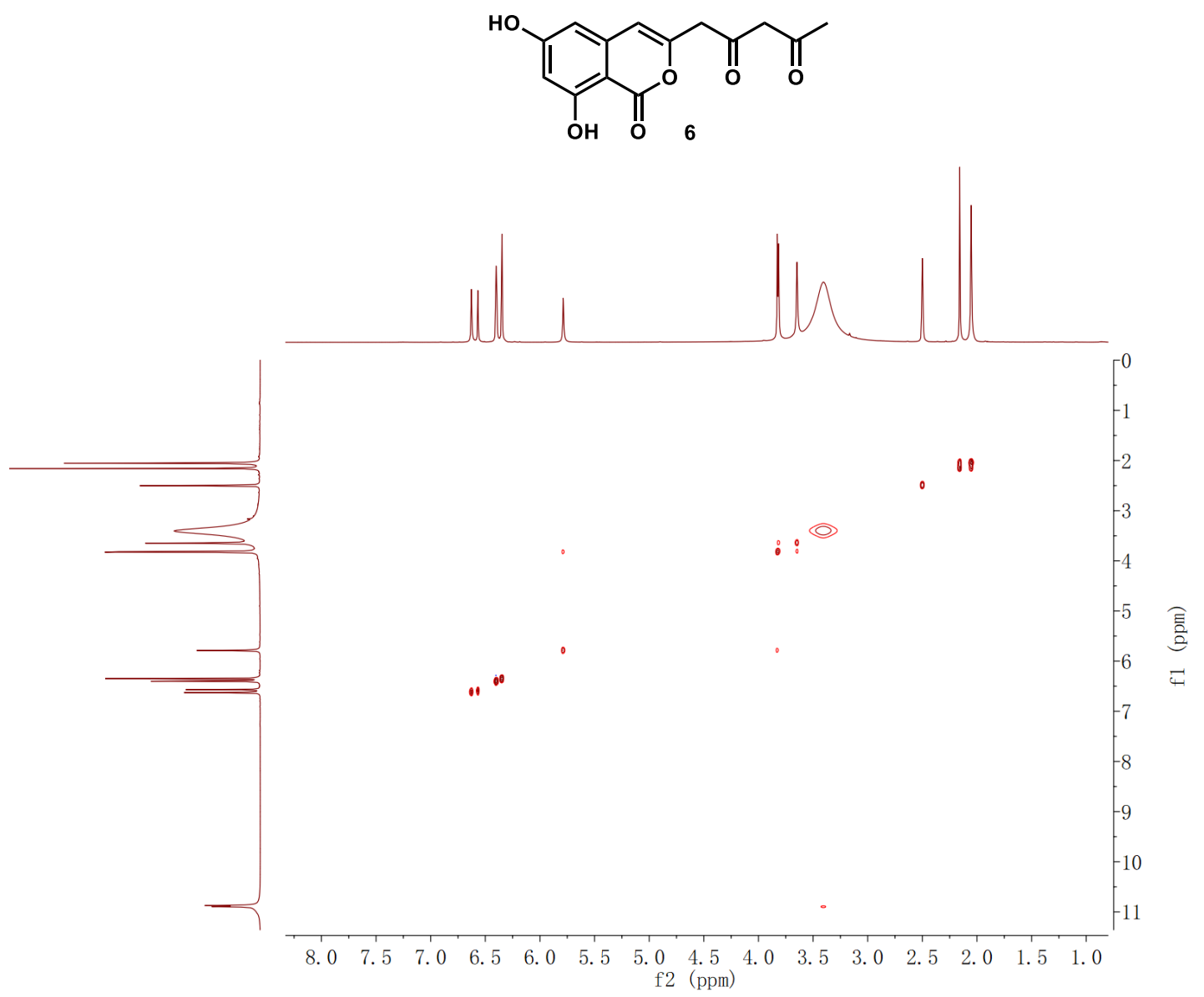

Figure S45. The ${ }^{1} \mathrm{H}-{ }^{1} \mathrm{H}$ COSY spectrum of compound 6 in DMSO- $d_{6}$. 

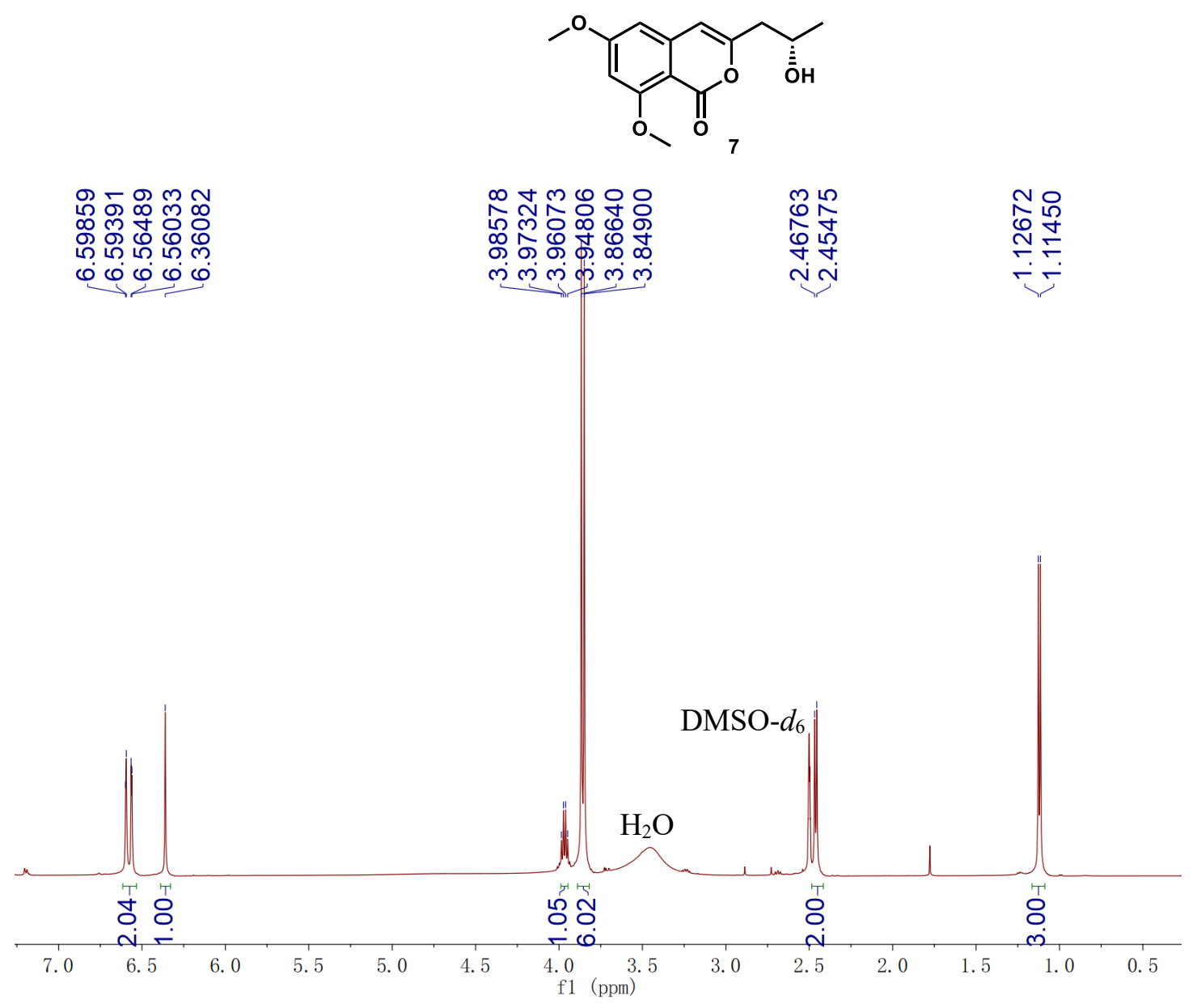

Figure S46. The ${ }^{1} \mathrm{H}$ NMR spectrum of compound 7 in DMSO- $d_{6}$. 

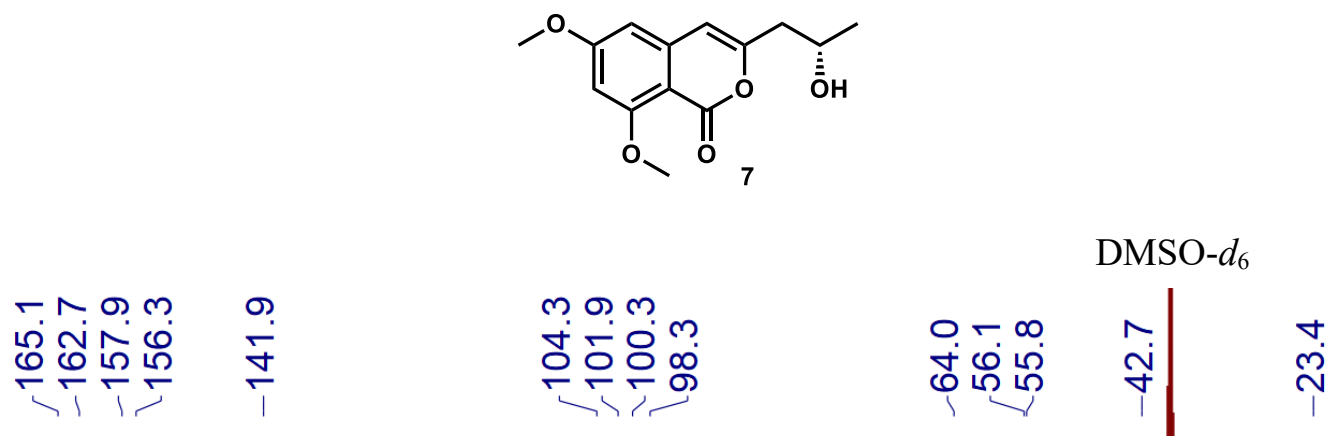

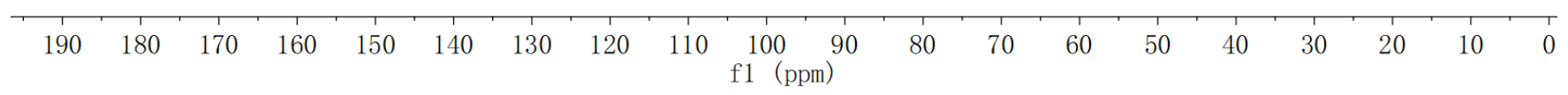

Figure S47. The ${ }^{13} \mathrm{C}$ NMR spectrum of compound 7 in DMSO- $d_{6}$. 

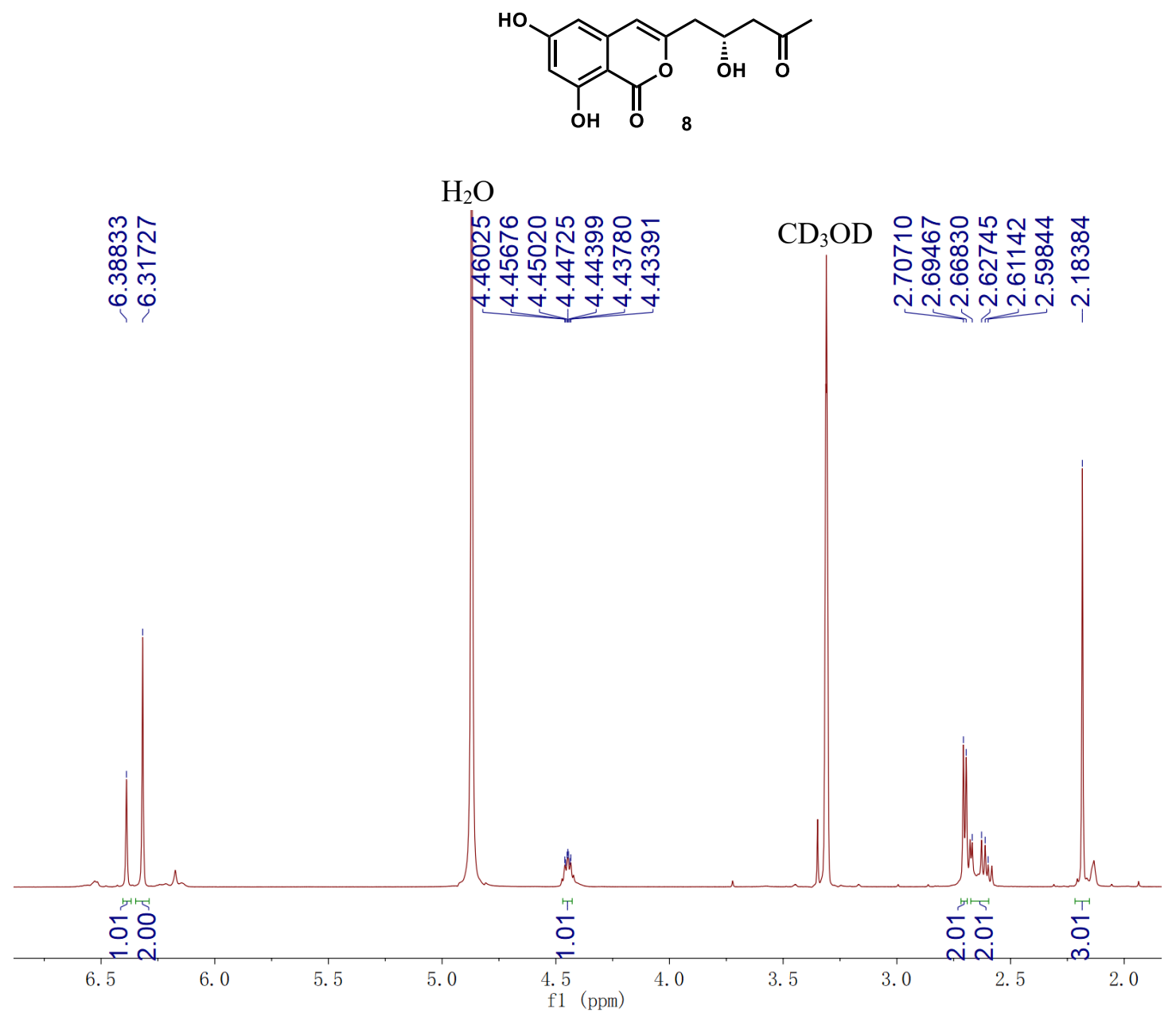

Figure S48. The ${ }^{1} \mathrm{H}$ NMR spectrum of compound 8 in $\mathrm{CD}_{3} \mathrm{OD}$. 

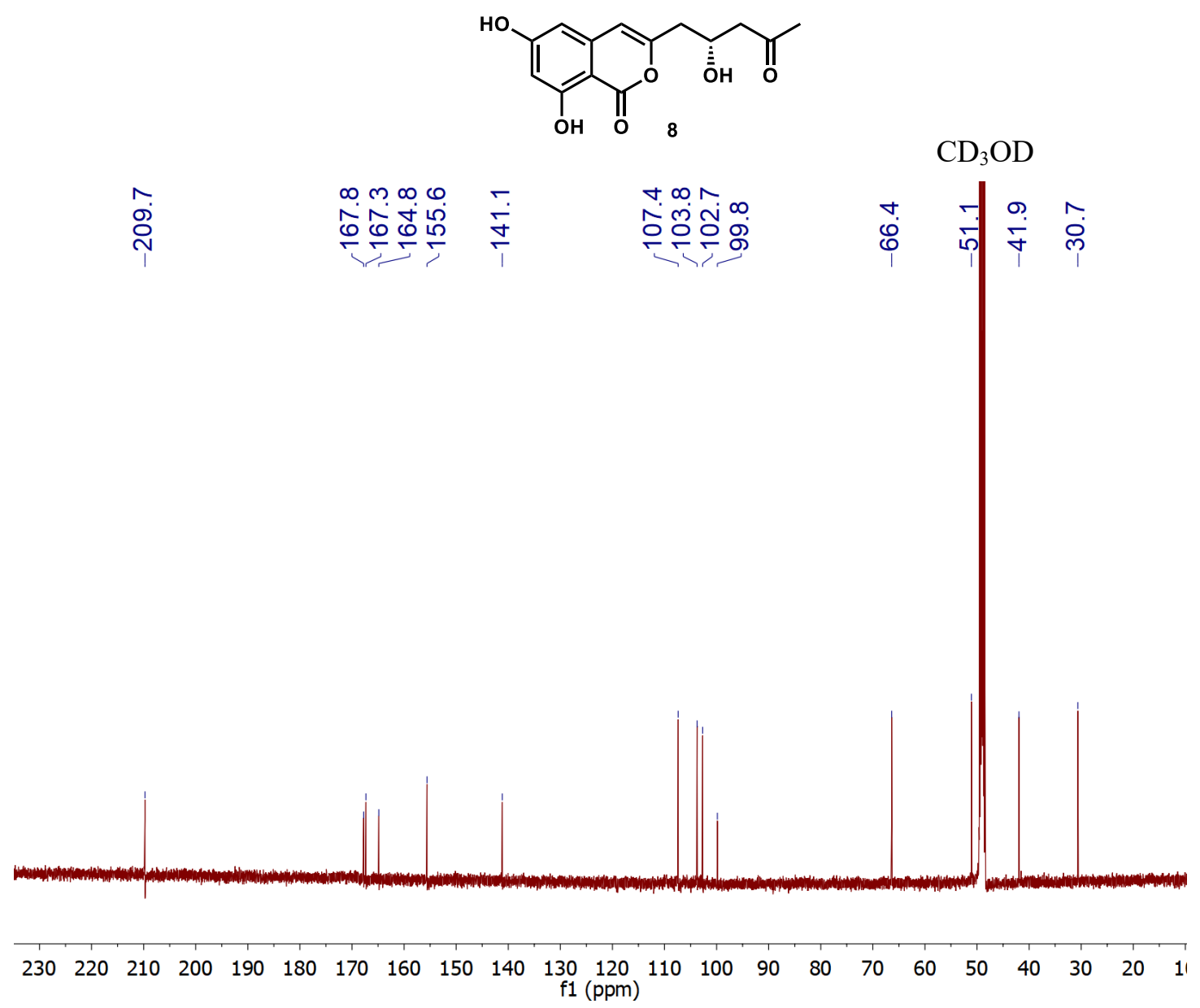

Figure S49. The ${ }^{13} \mathrm{C}$ NMR spectrum of compound $\mathbf{8}$ in $\mathrm{CD}_{3} \mathrm{OD}$. 

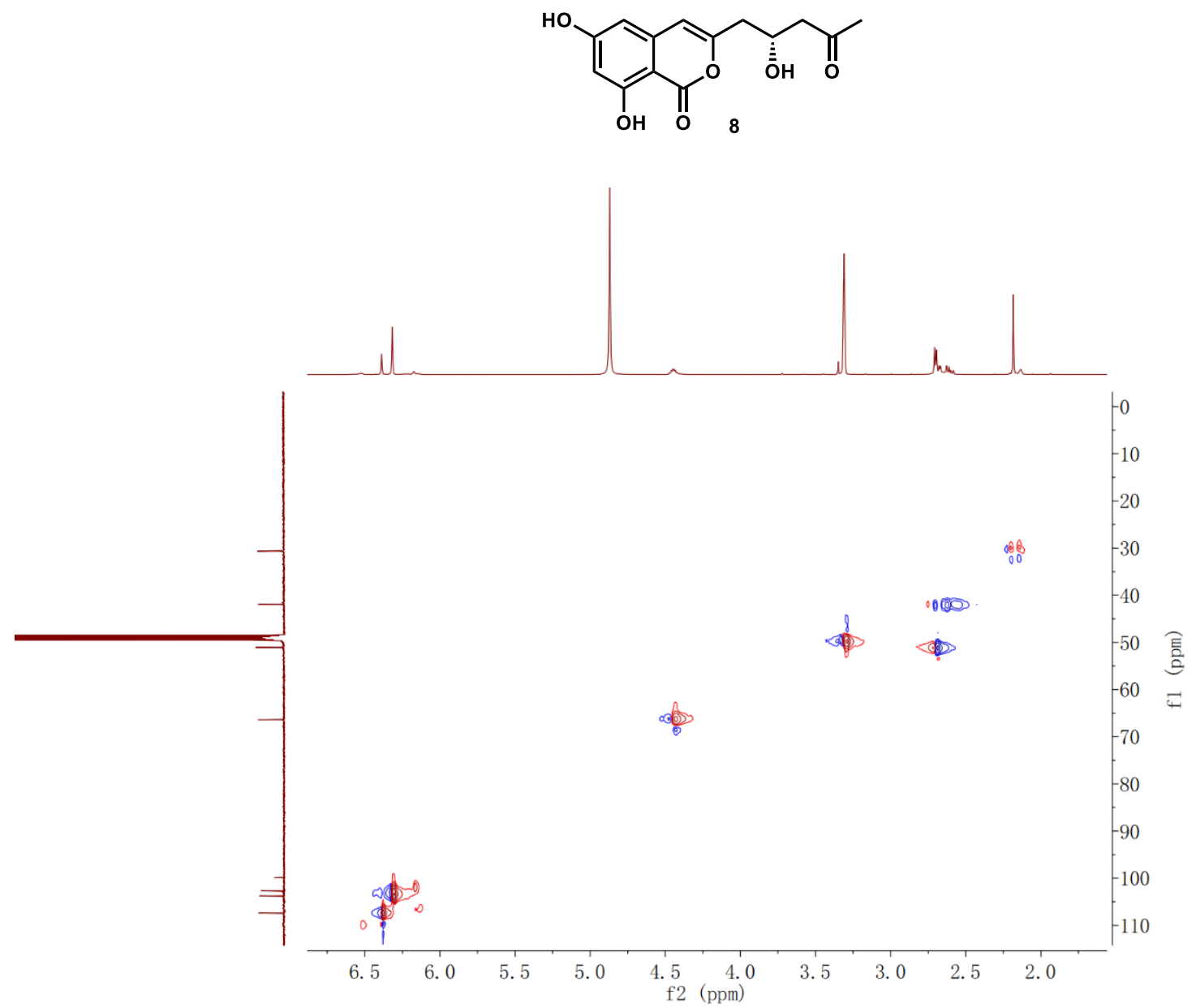

Figure S50. The HSQC spectrum of compound 8 in $\mathrm{CD}_{3} \mathrm{OD}$. 

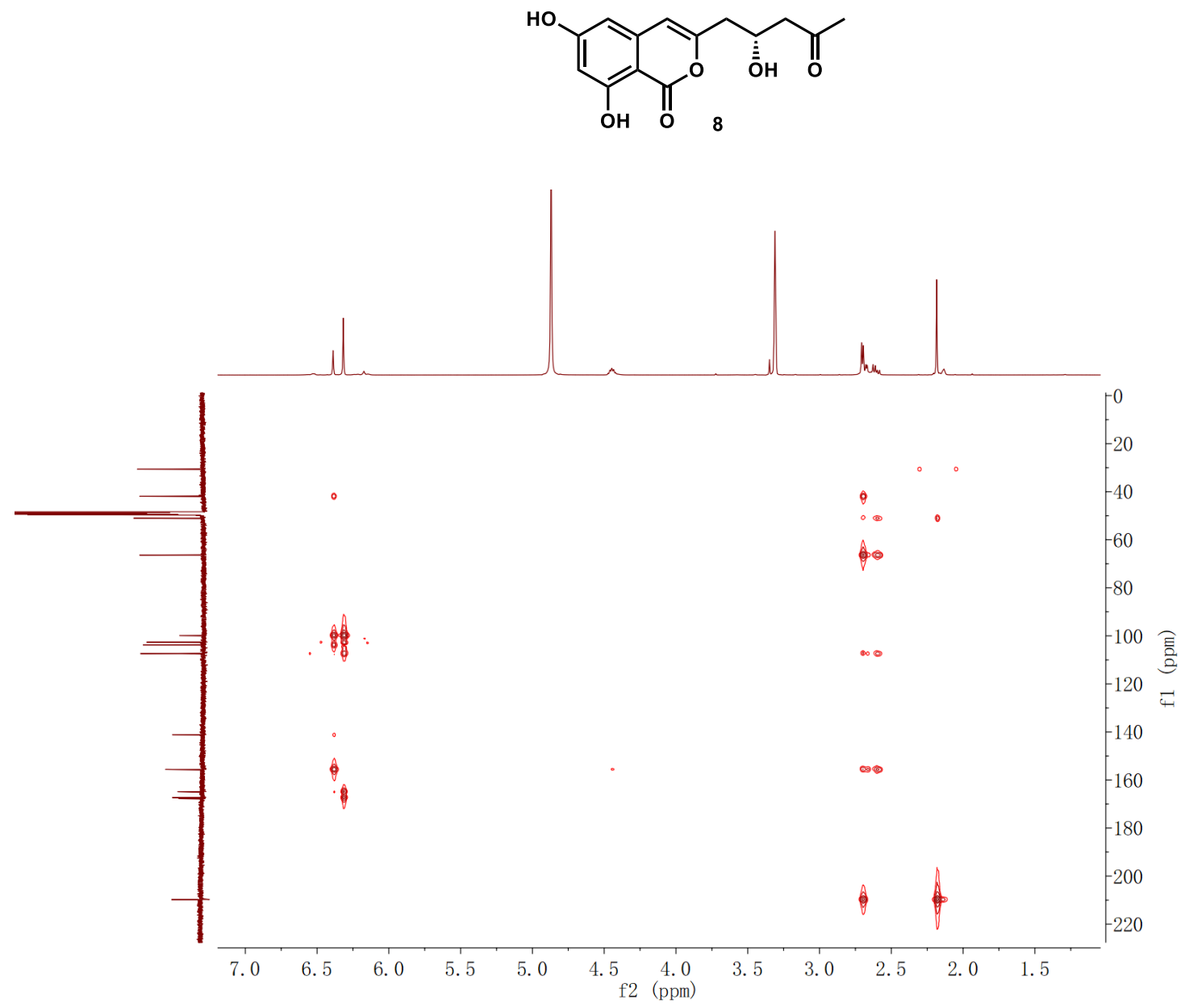

Figure S51. The HMBC spectrum of compound 8 in $\mathrm{CD}_{3} \mathrm{OD}$. 

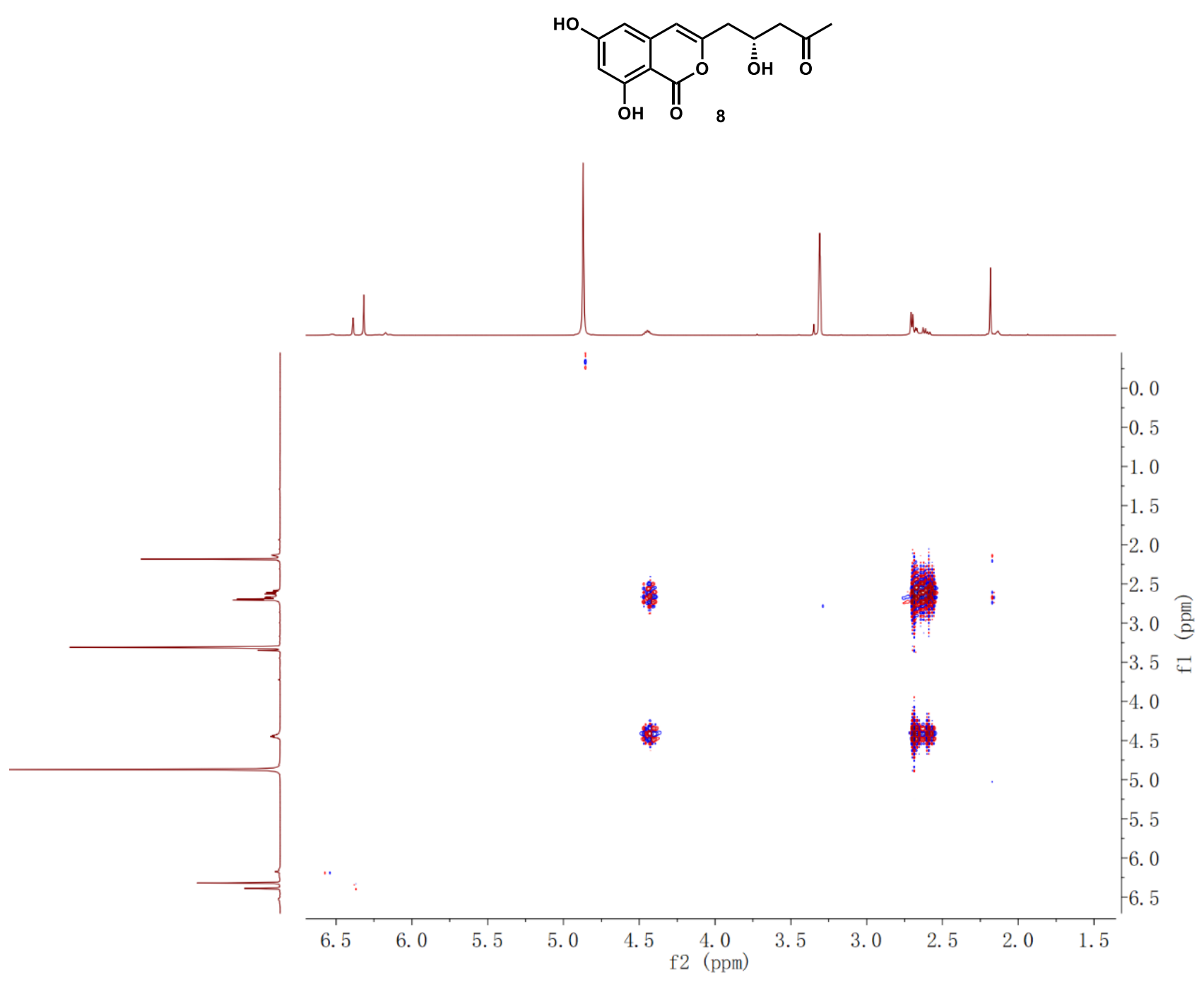

Figure S52. The ${ }^{1} \mathrm{H}-{ }^{1} \mathrm{H}$ COSY spectrum of compound $\mathbf{8}$ in $\mathrm{CD}_{3} \mathrm{OD}$ 

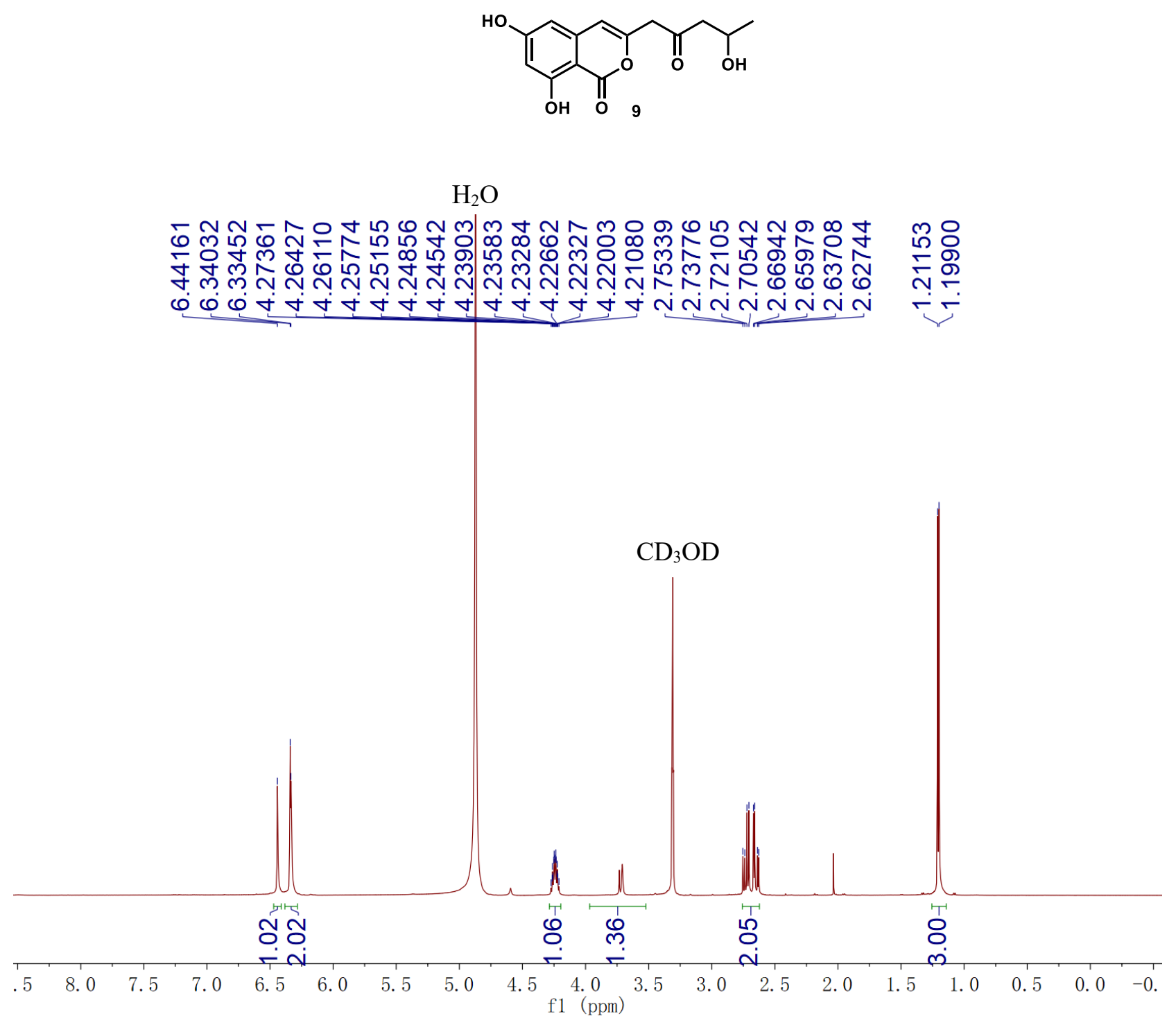

Figure S53. The ${ }^{1} \mathrm{H}$ NMR spectrum of compound 9 in $\mathrm{CD}_{3} \mathrm{OD}$. 

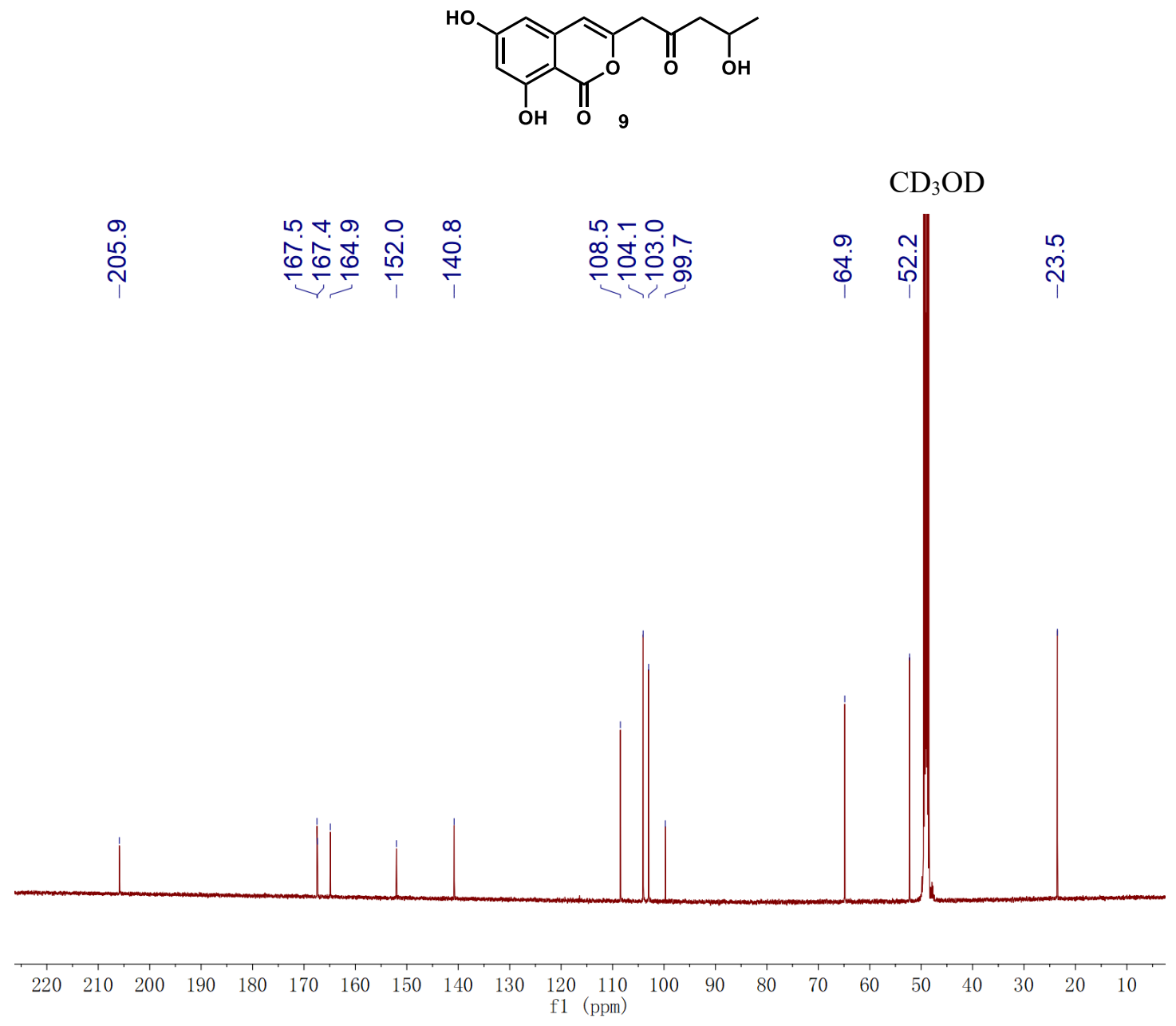

Figure S54. The ${ }^{13} \mathrm{C}$ NMR spectrum of compound 9 in $\mathrm{CD}_{3} \mathrm{OD}$. 

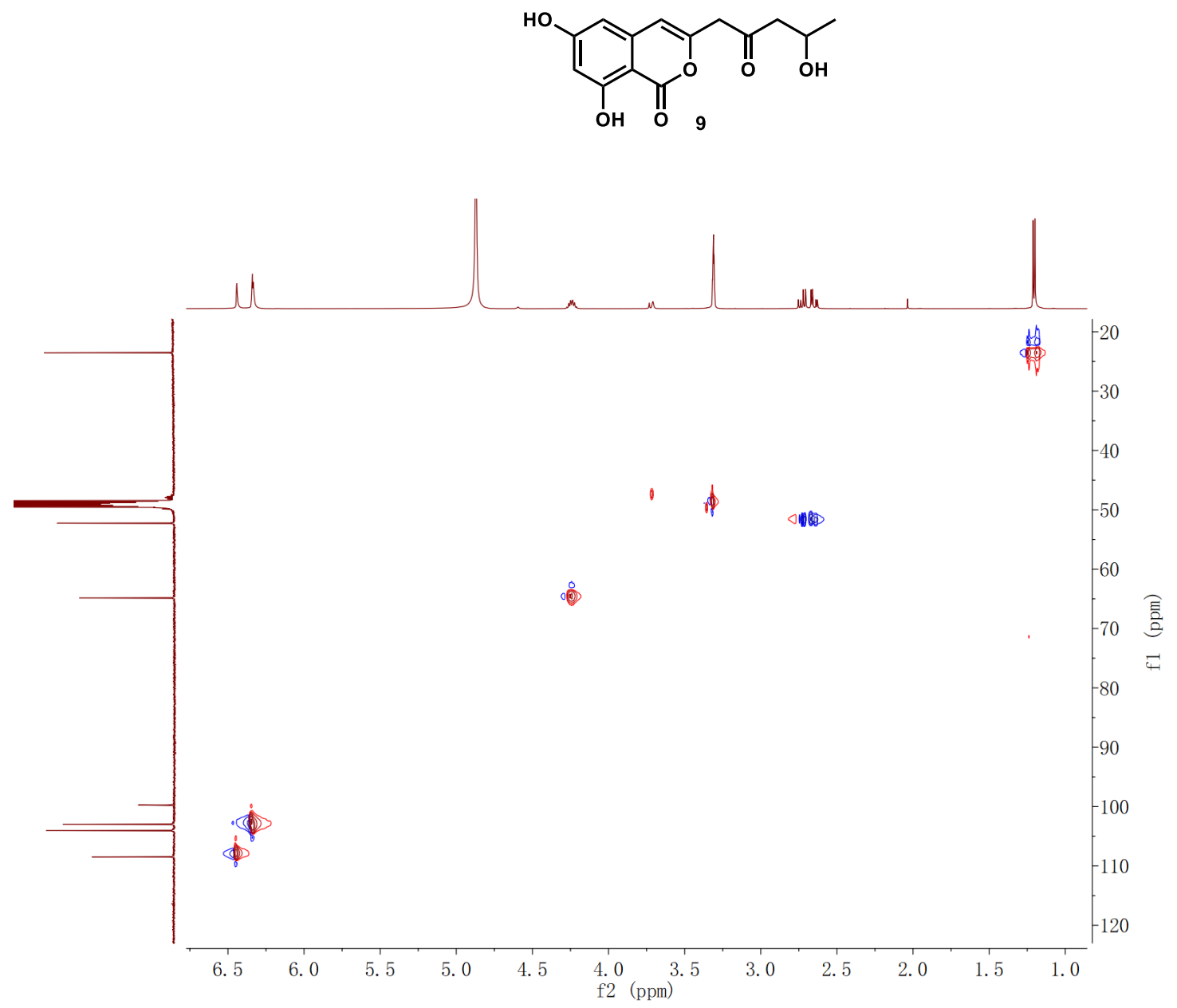

Figure S55. The HSQC spectrum of compound 9 in $\mathrm{CD}_{3} \mathrm{OD}$. 

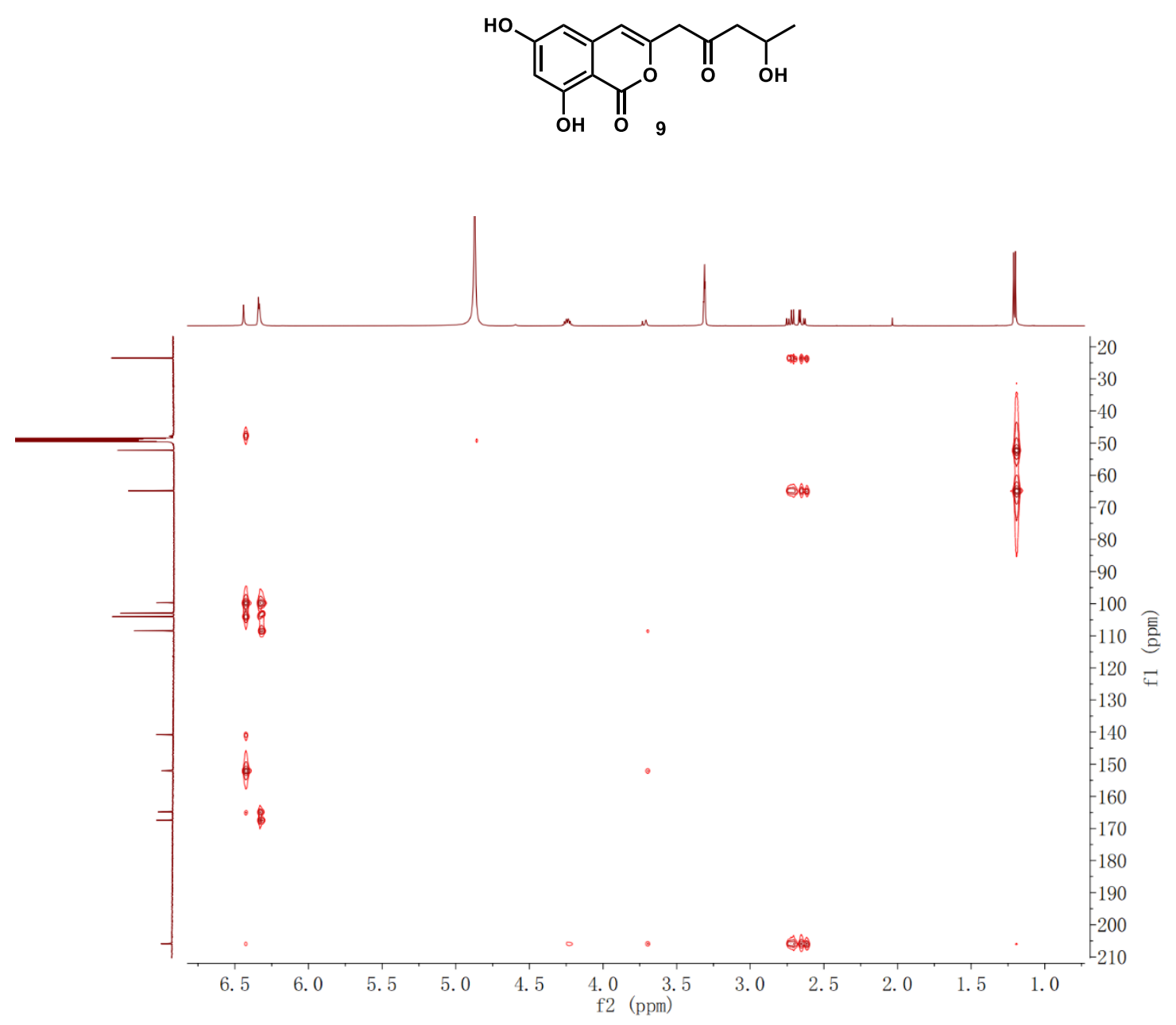

Figure S56. The $\mathrm{HMBC}$ spectrum of compound 9 in $\mathrm{CD}_{3} \mathrm{OD}$. 

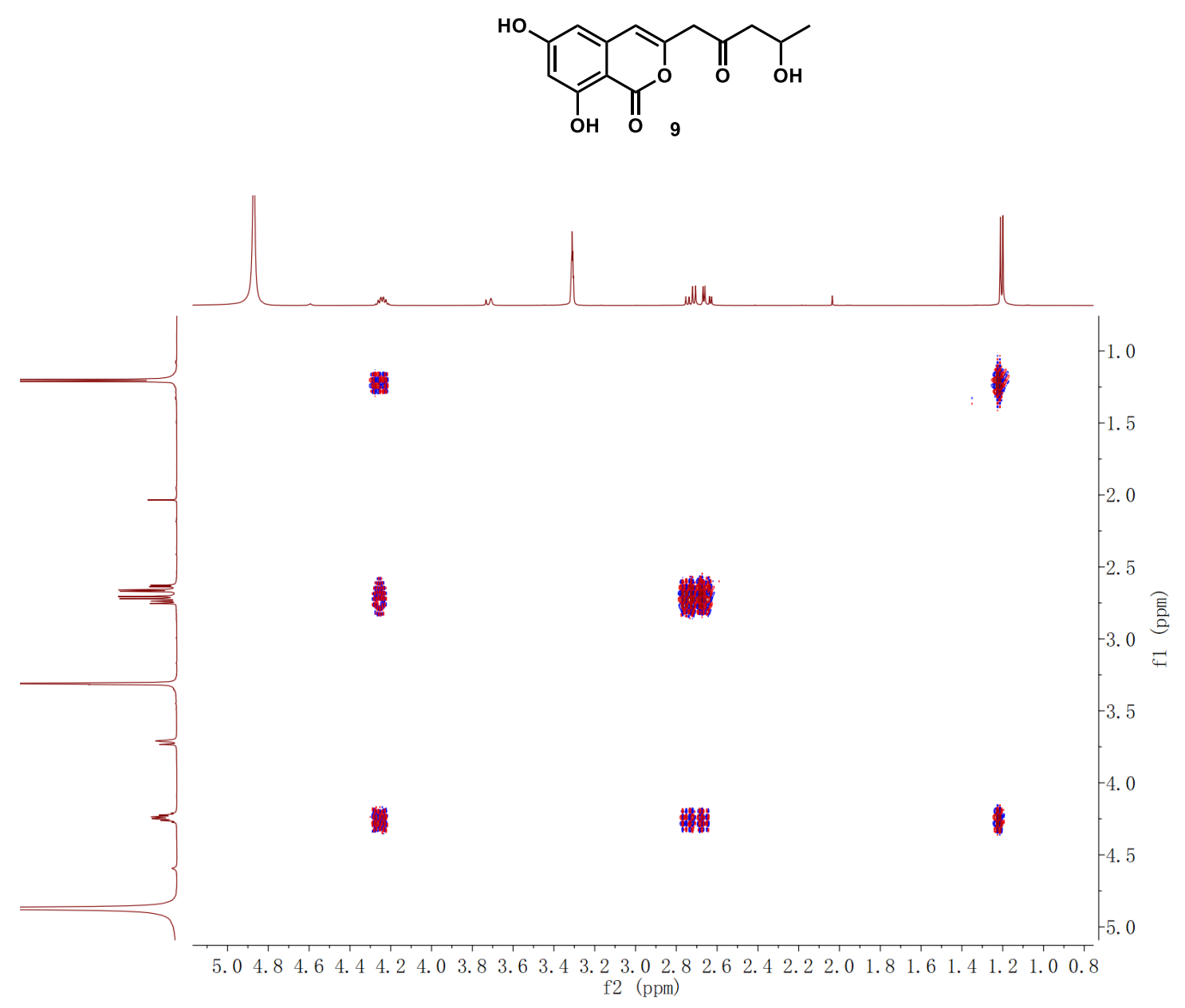

Figure S57. The ${ }^{1} \mathrm{H}-{ }^{1} \mathrm{H}$ COSY spectrum of compound 9 in $\mathrm{CD}_{3} \mathrm{OD}$. 

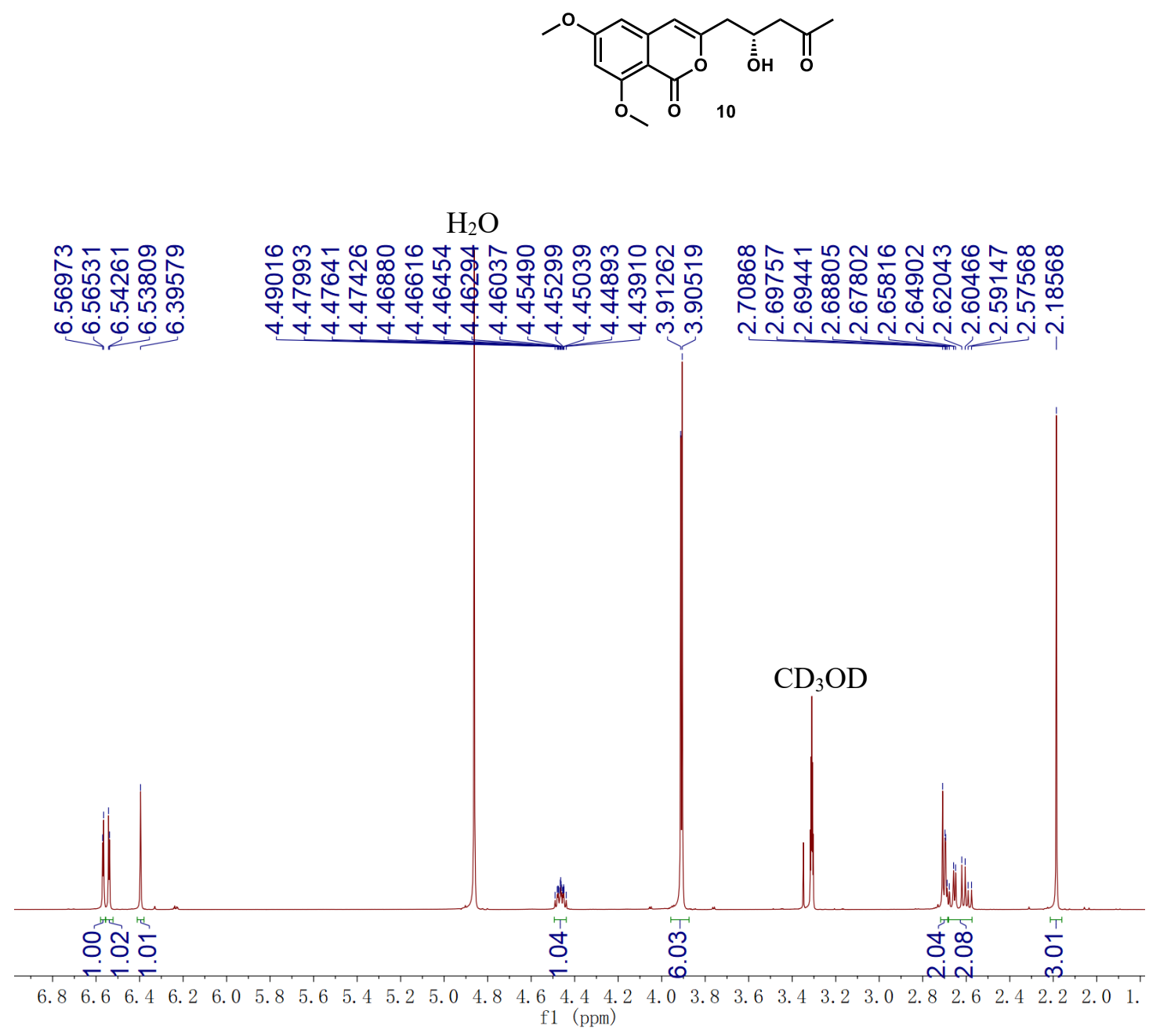

Figure S58. The ${ }^{1} \mathrm{H}$ NMR spectrum of compound $\mathbf{1 0}$ in $\mathrm{CD}_{3} \mathrm{OD}$. 

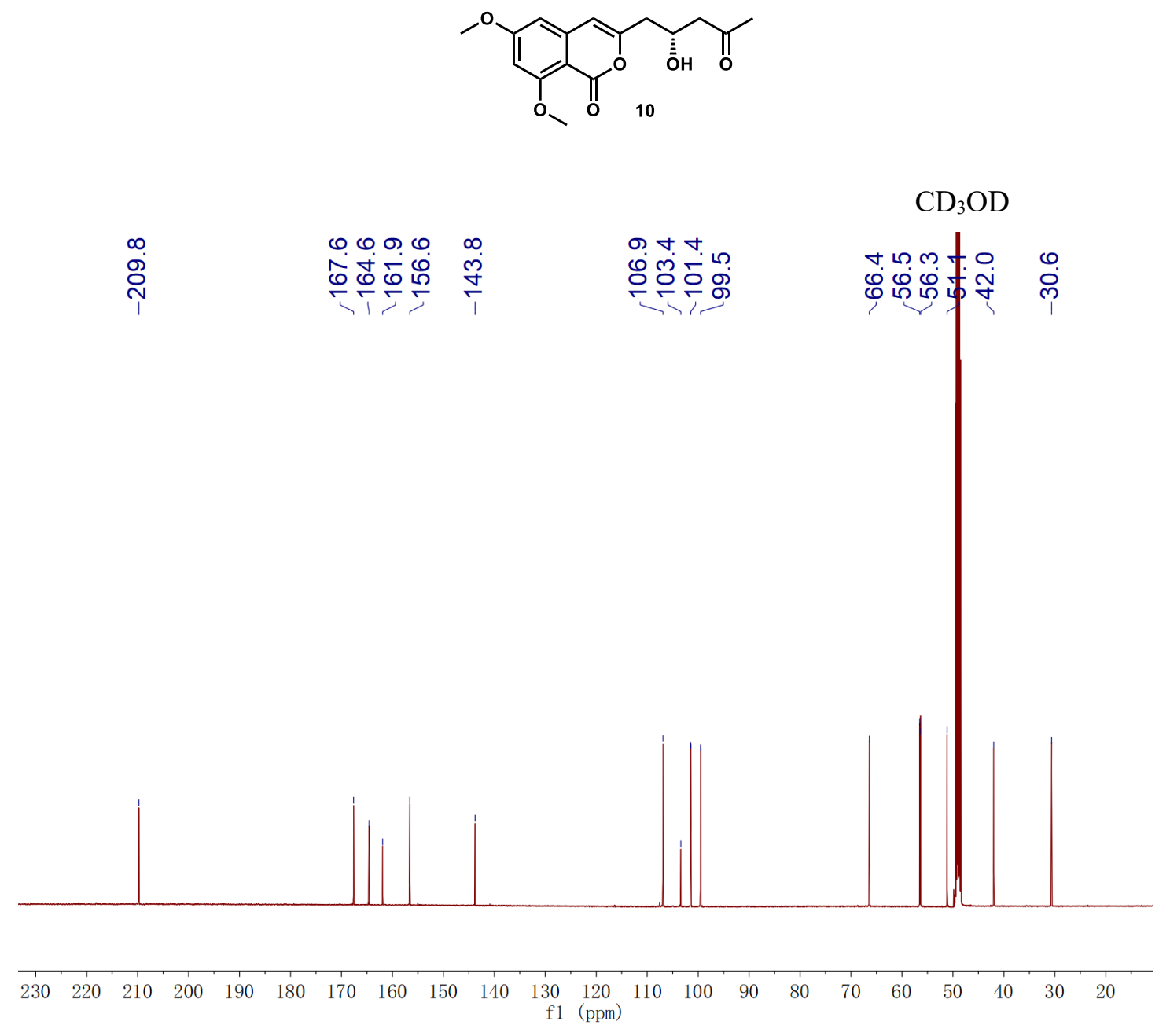

Figure S59. The ${ }^{13} \mathrm{C}$ NMR spectrum of compound $\mathbf{1 0}$ in $\mathrm{CD}_{3} \mathrm{OD}$. 

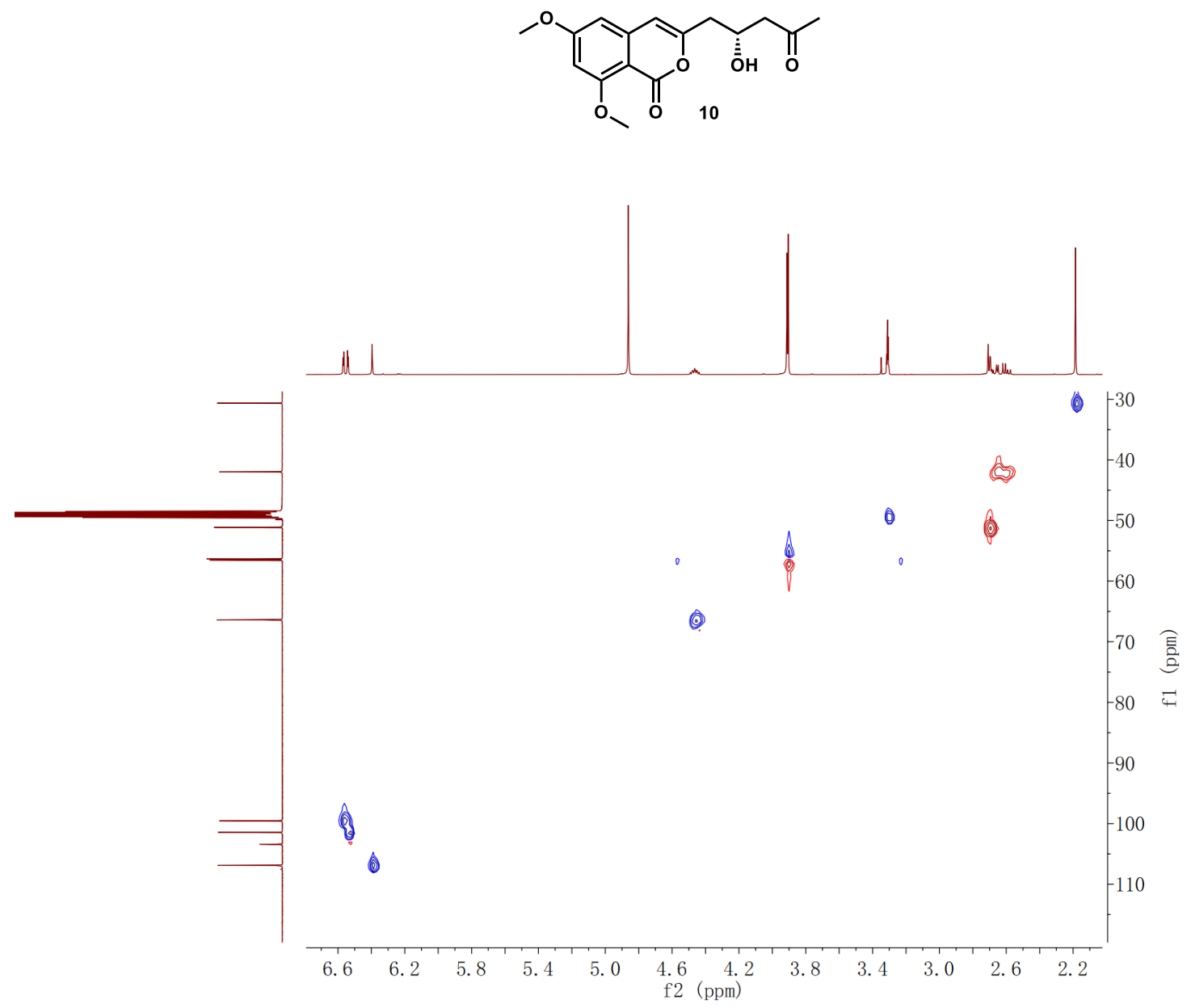

Figure S60. The HSQC spectrum of compound $\mathbf{1 0}$ in $\mathrm{CD}_{3} \mathrm{OD}$. 

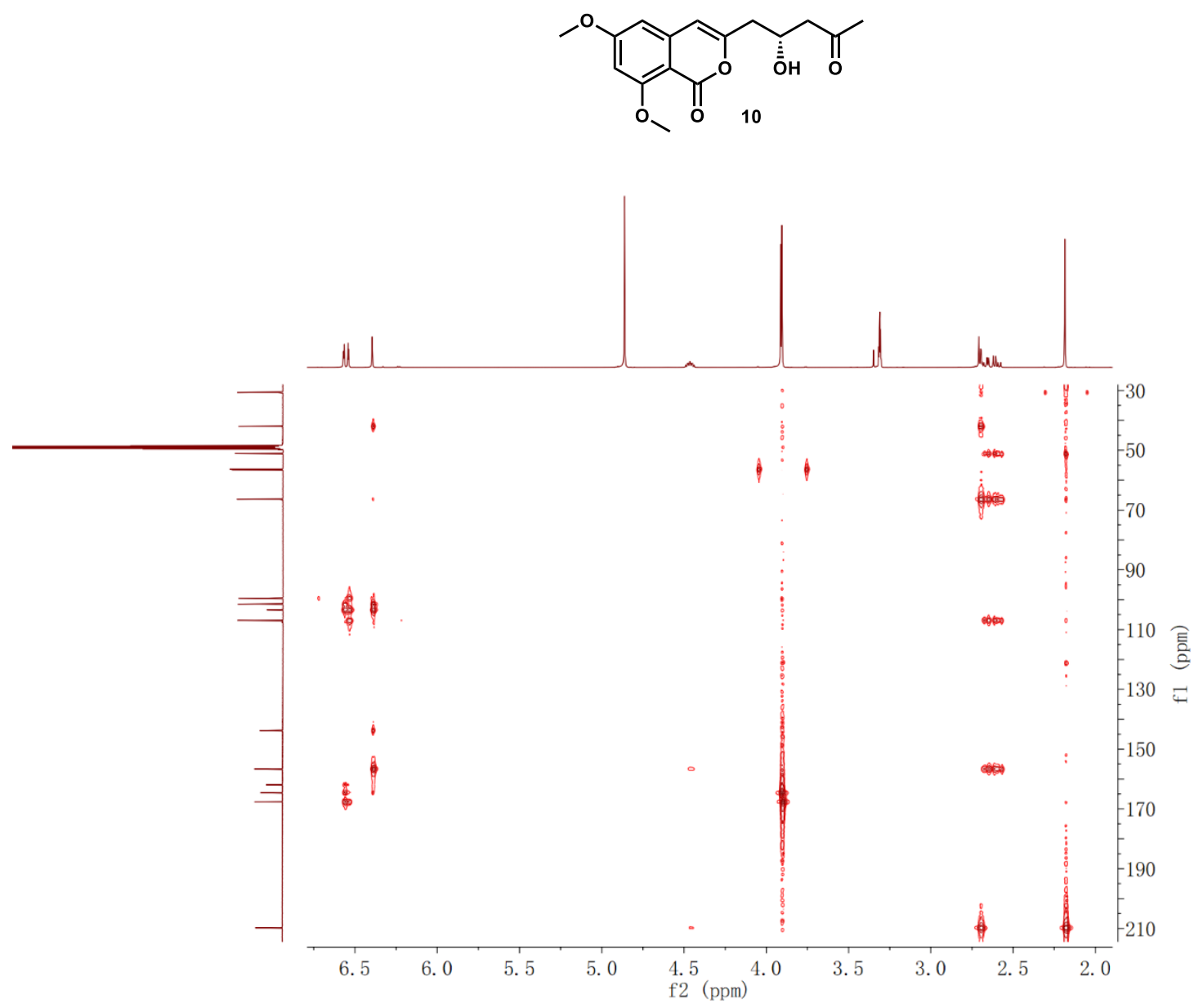

Figure S61. The HMBC spectrum of compound 10 in $\mathrm{CD}_{3} \mathrm{OD}$. 

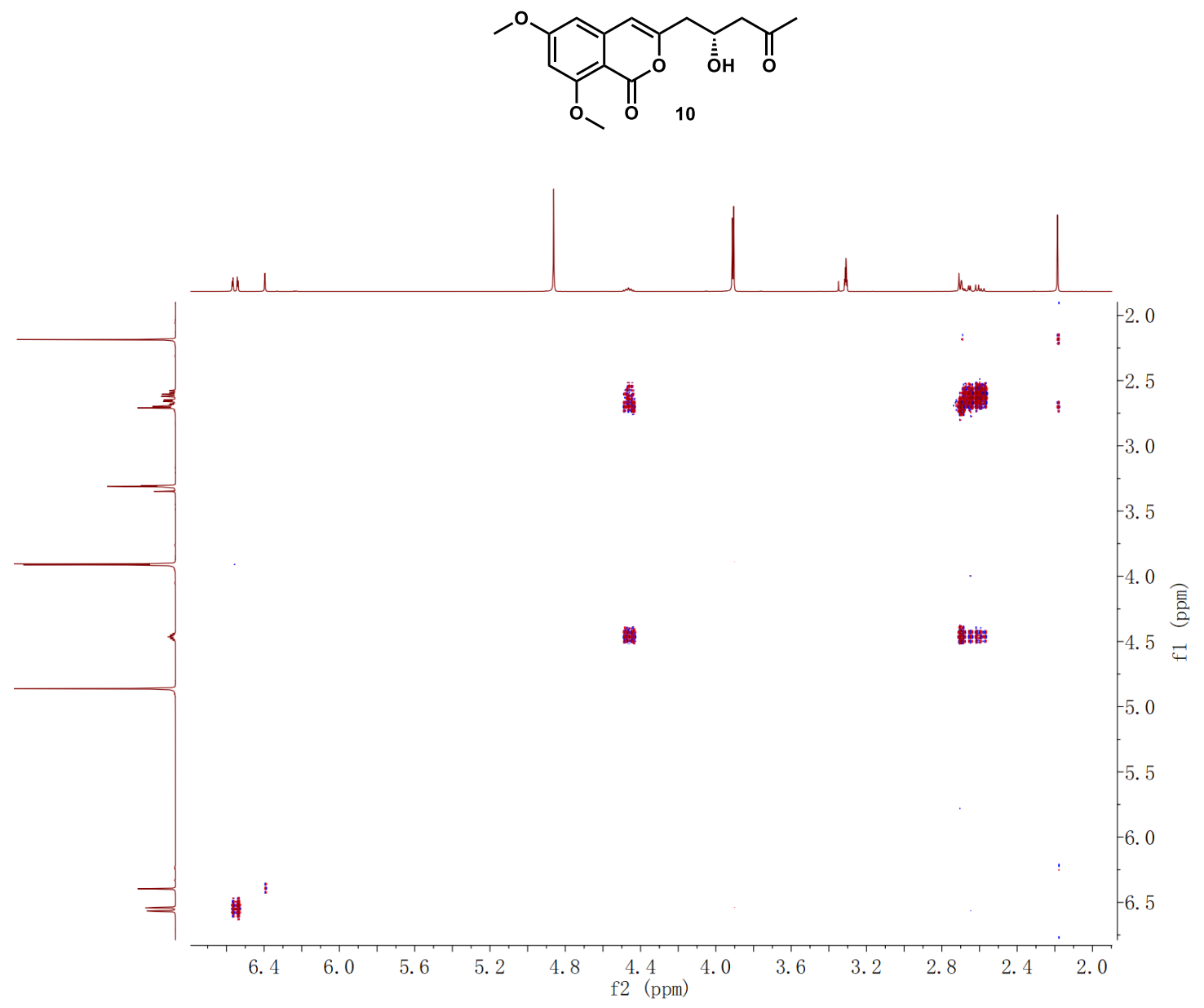

Figure S62. The ${ }^{1} \mathrm{H}-{ }^{1} \mathrm{H}$ COSY spectrum of compound $\mathbf{1 0}$ in $\mathrm{CD}_{3} \mathrm{OD}$. 

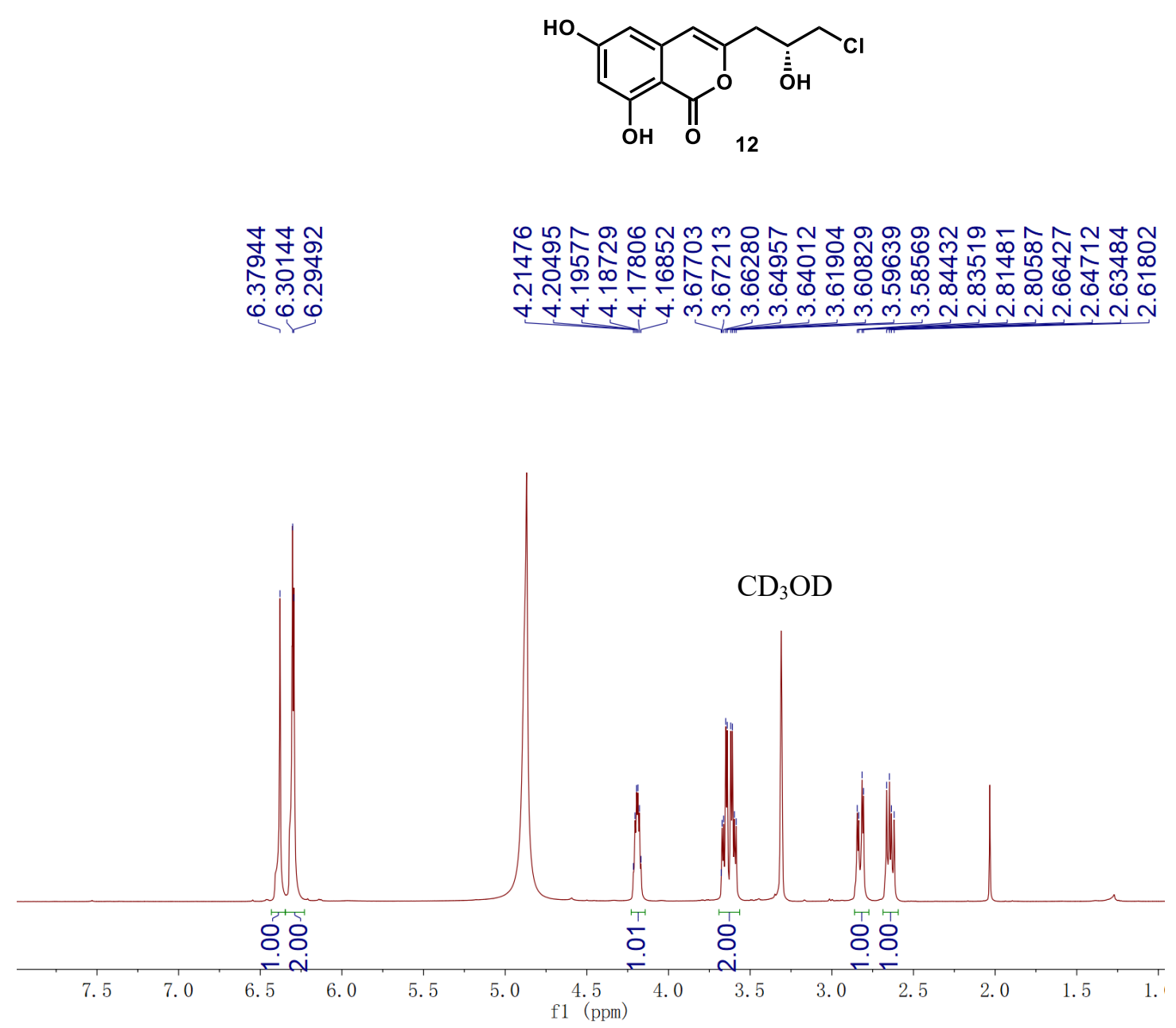

Figure S63. The ${ }^{1} \mathrm{H}$ NMR spectrum of compound 12 in $\mathrm{CD}_{3} \mathrm{OD}$. 

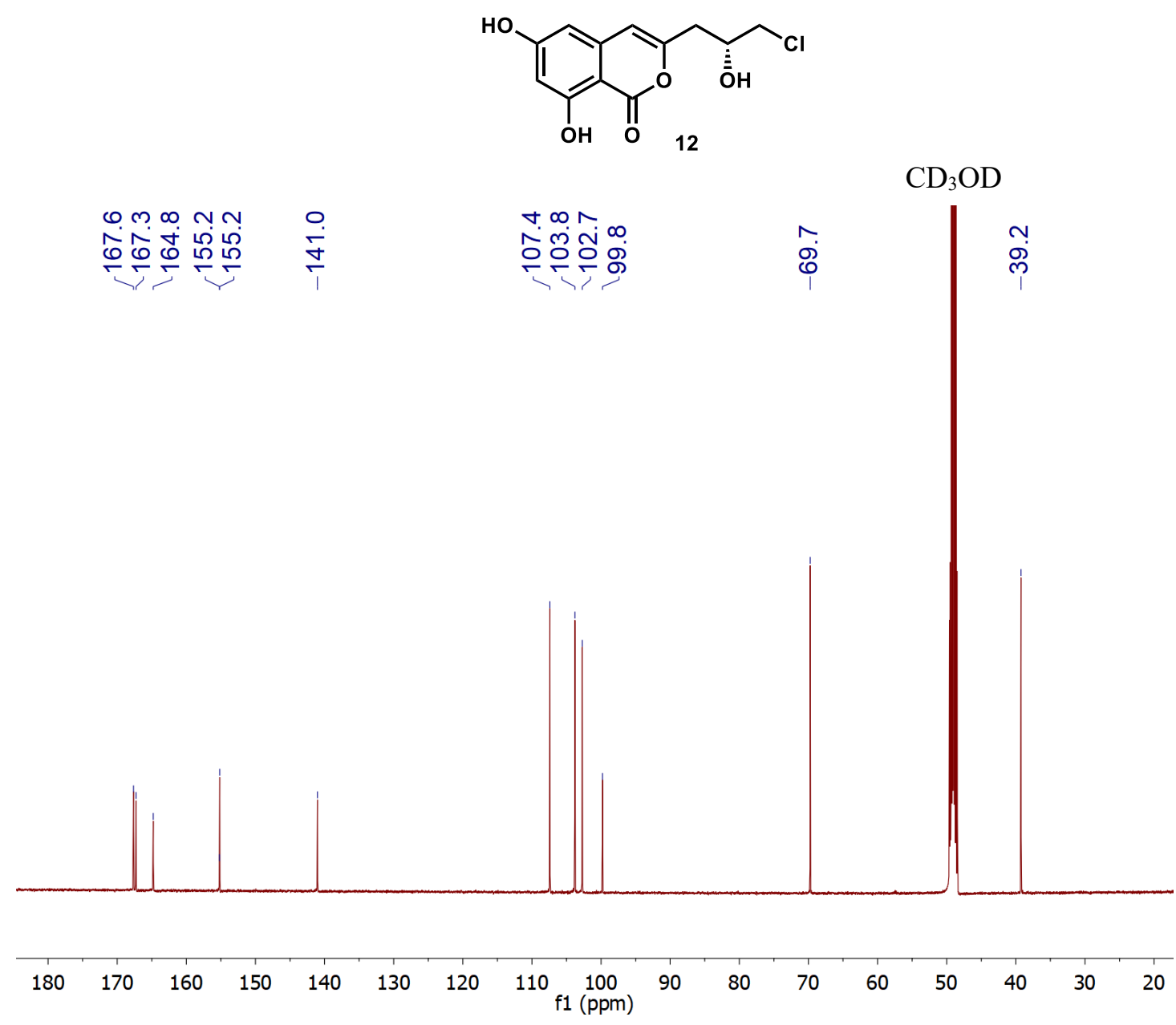

Figure S64. The ${ }^{13} \mathrm{C}$ NMR spectrum of compound 12 in $\mathrm{CD}_{3} \mathrm{OD}$. 


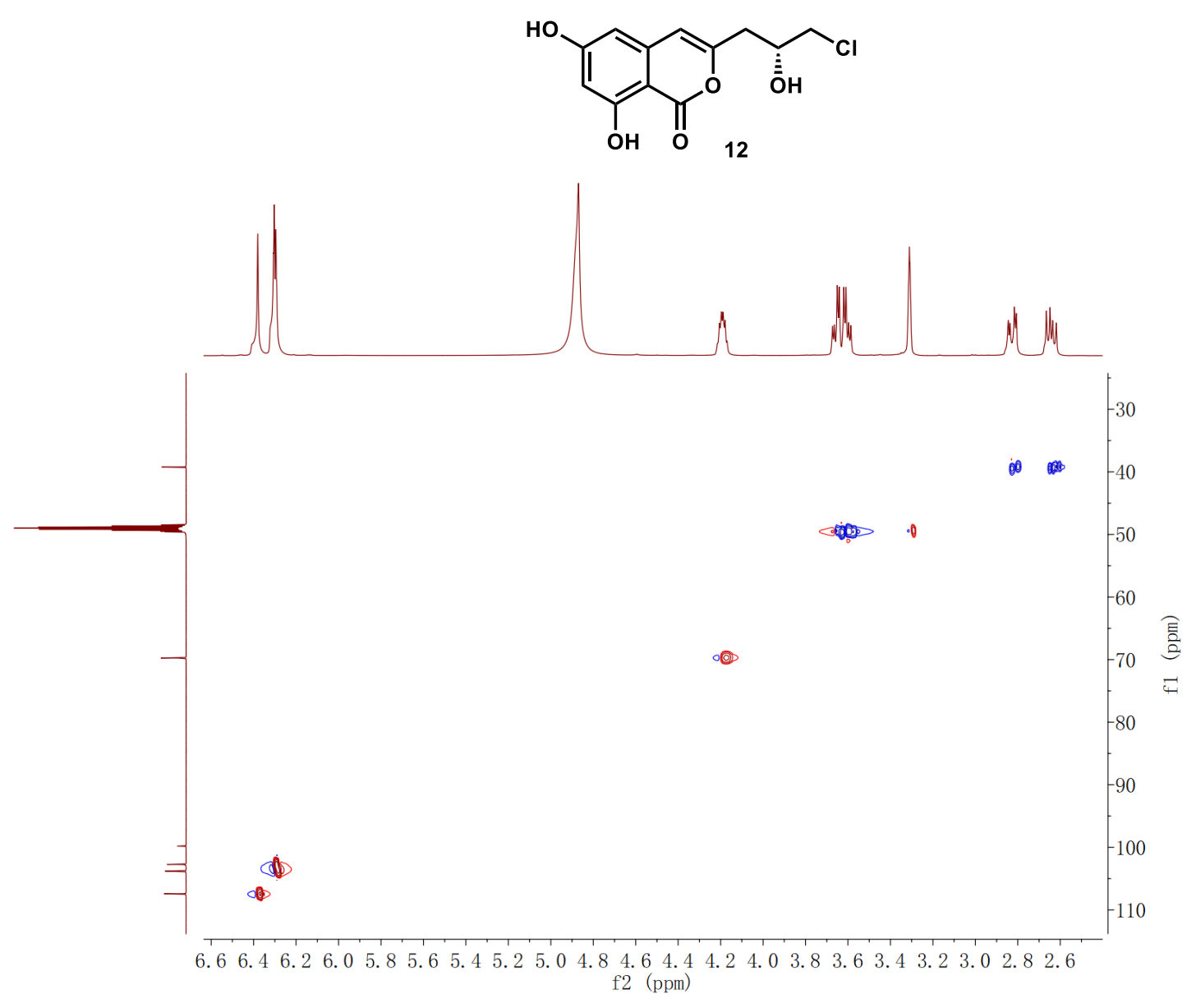

Figure S65. The HSQC spectrum of compound 12 in $\mathrm{CD}_{3} \mathrm{OD}$. 

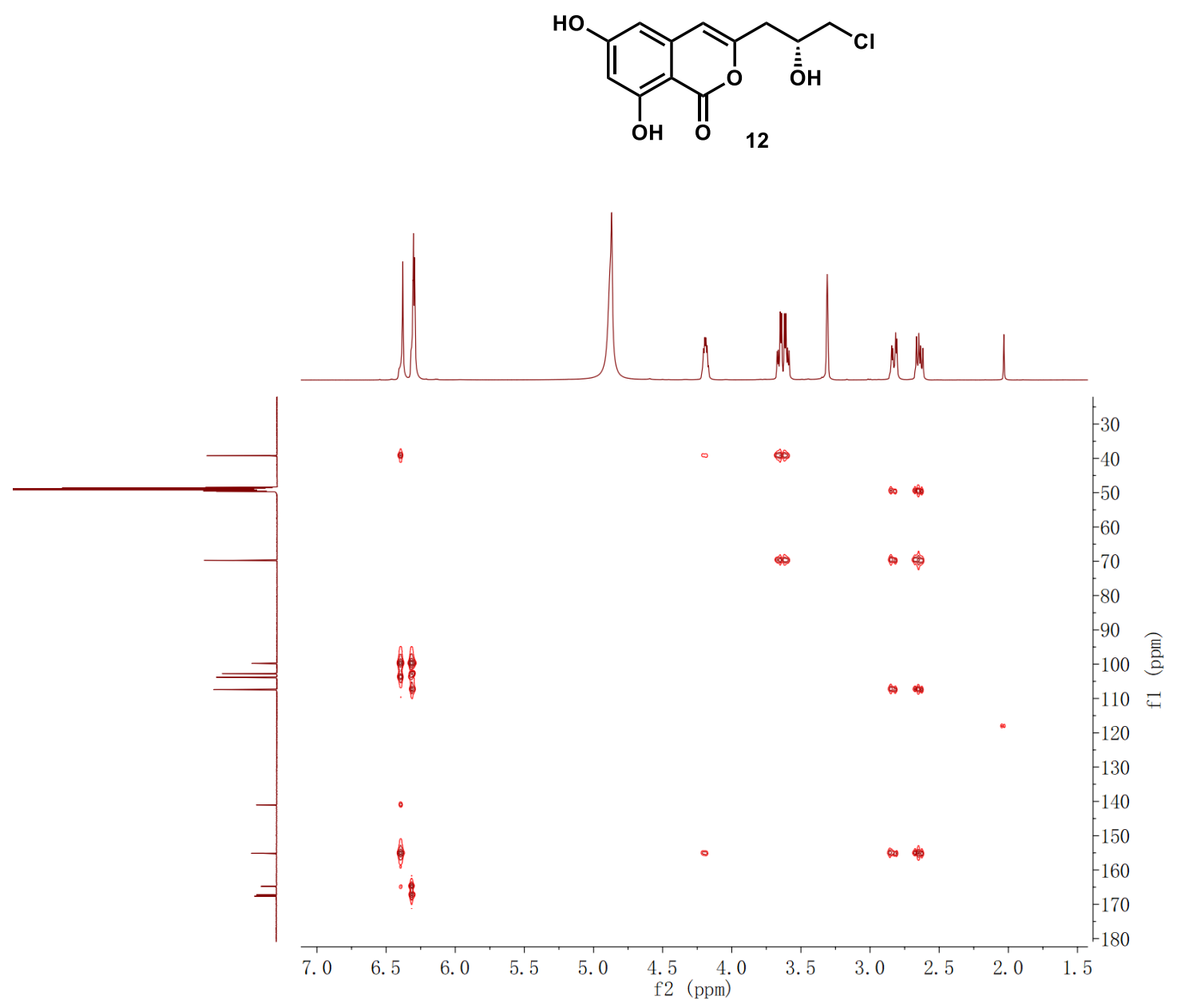

Figure S66. The HMBC spectrum of compound 12 in $\mathrm{CD}_{3} \mathrm{OD}$. 


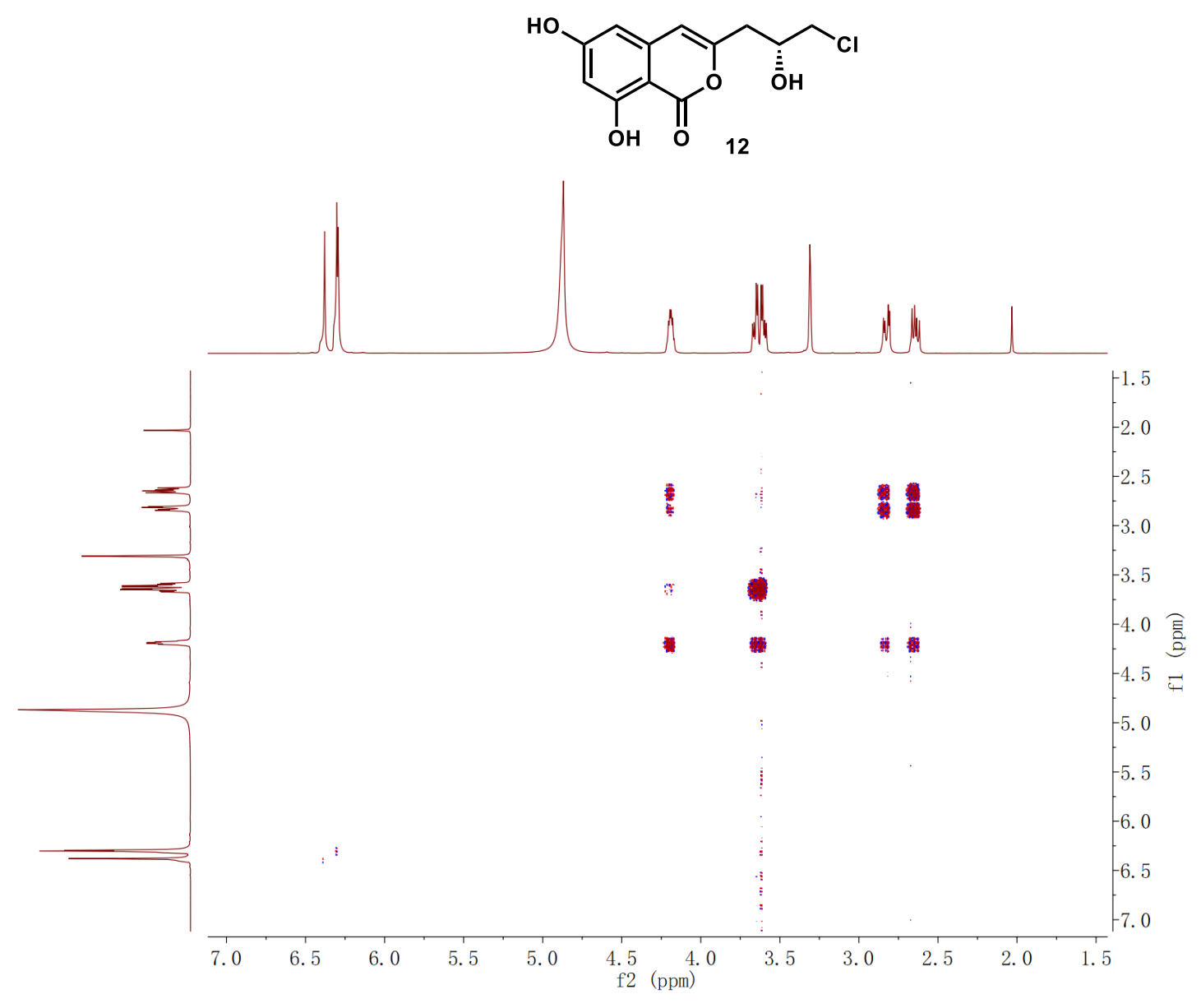

Figure S67. The ${ }^{1} \mathrm{H}-{ }^{1} \mathrm{H}$ COSY spectrum of compound 12 in $\mathrm{CD}_{3} \mathrm{OD}$. 

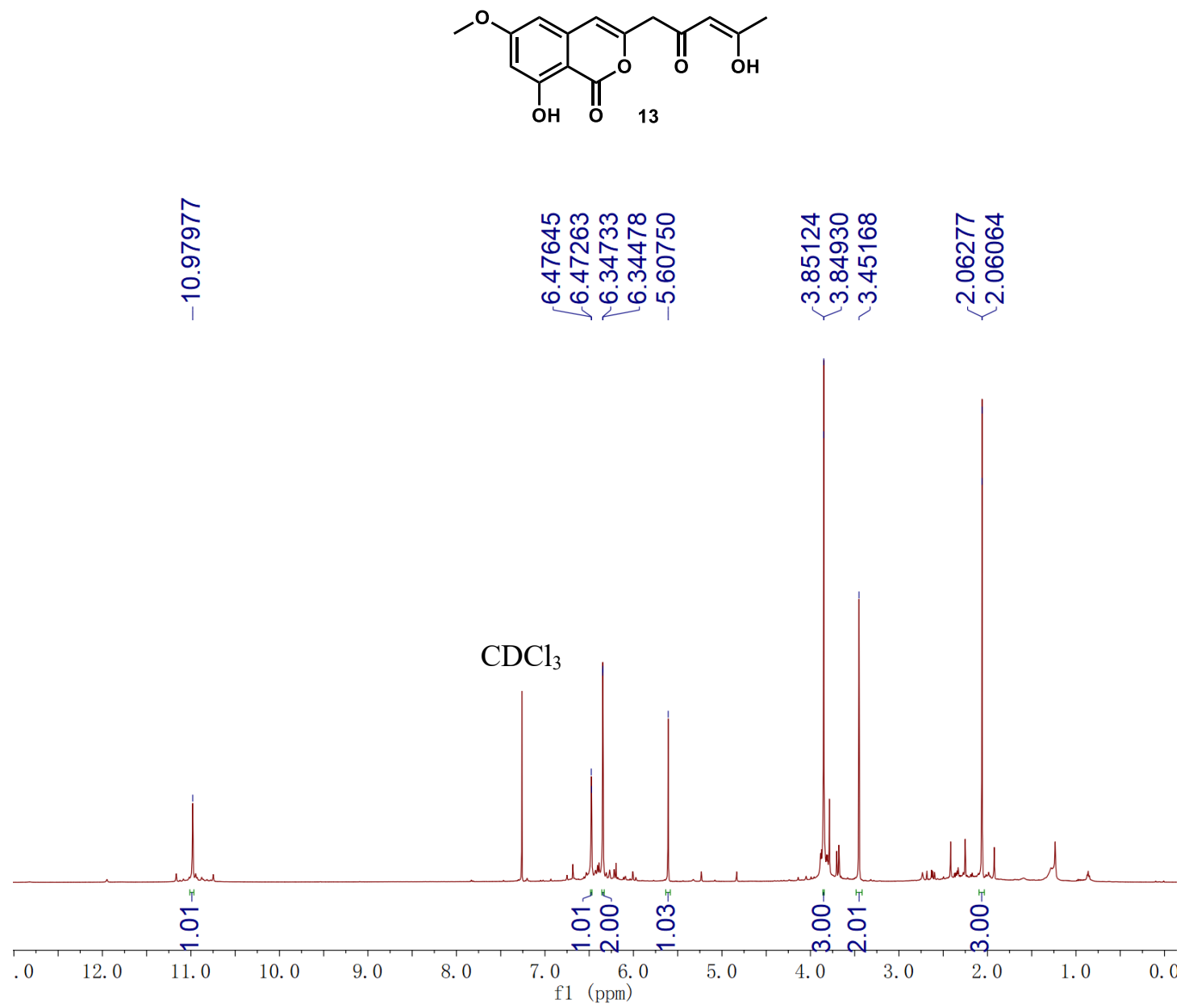

Figure S68. The ${ }^{1} \mathrm{H}$ NMR spectrum of compound 13 in $\mathrm{CDCl}_{3}$. 

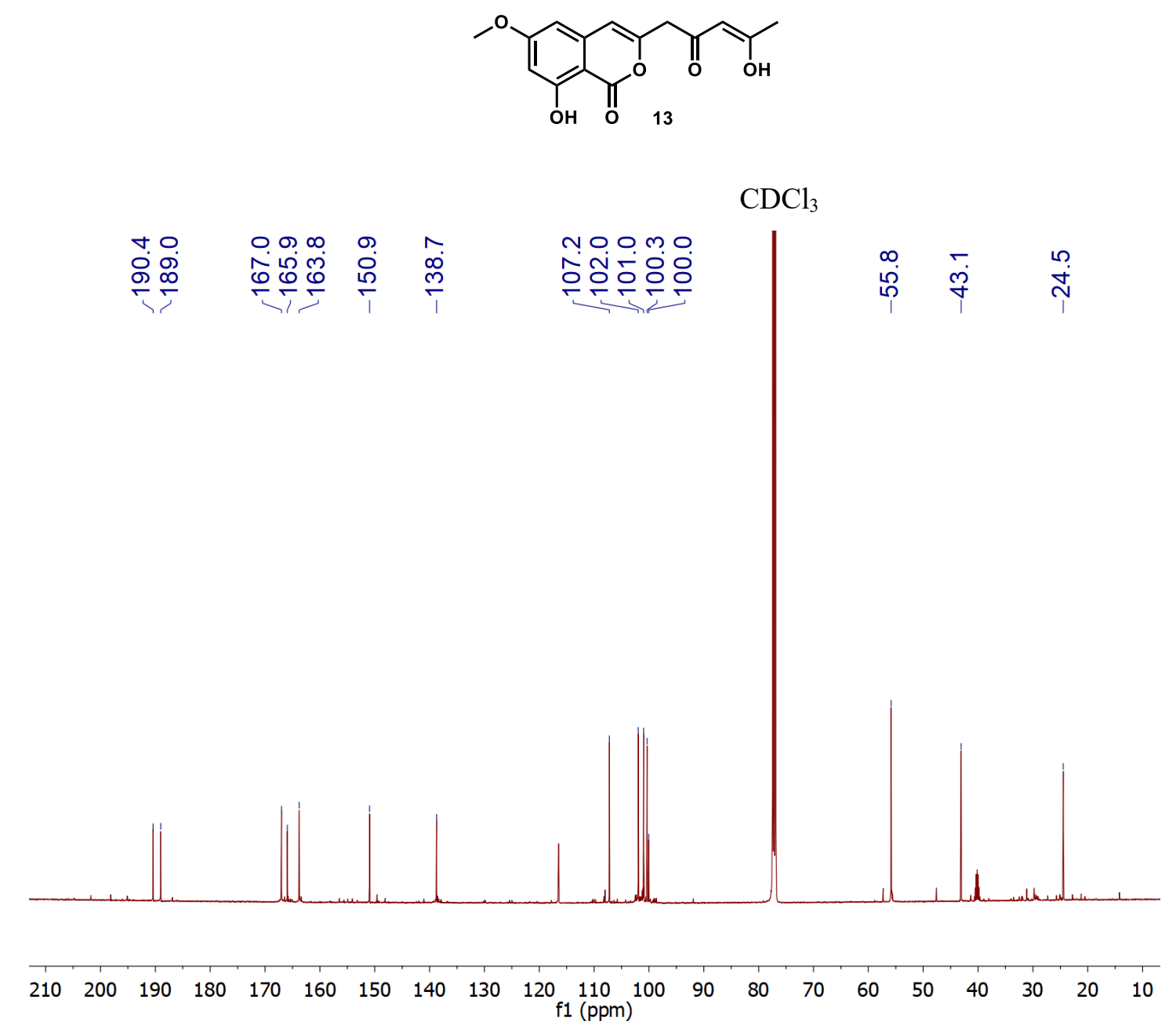

Figure S69. The ${ }^{13} \mathrm{C}$ NMR spectrum of compound 13 in $\mathrm{CDCl}_{3}$. 

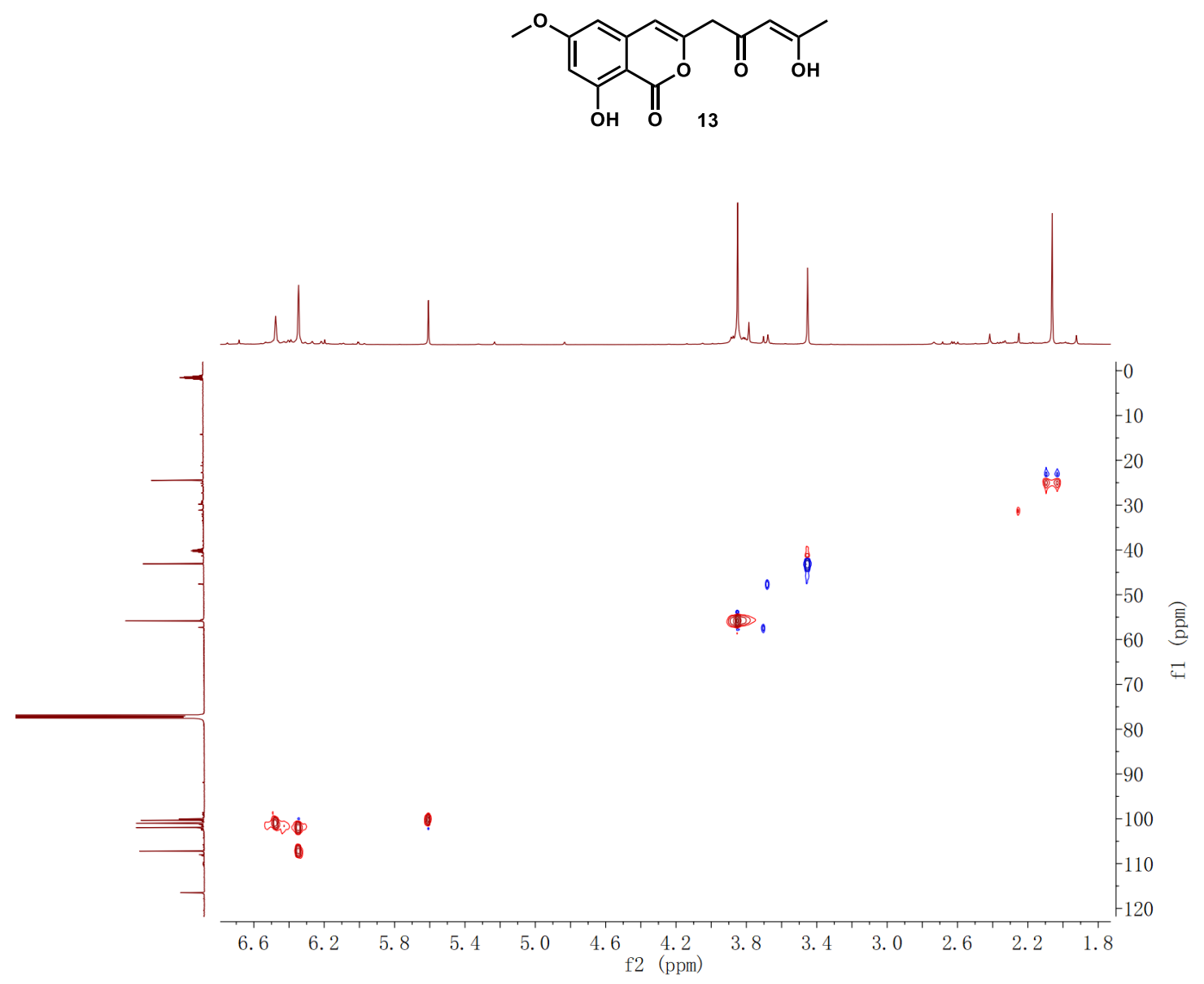

Figure S70. The HSQC spectrum of compound 13 in $\mathrm{CDCl}_{3}$. 

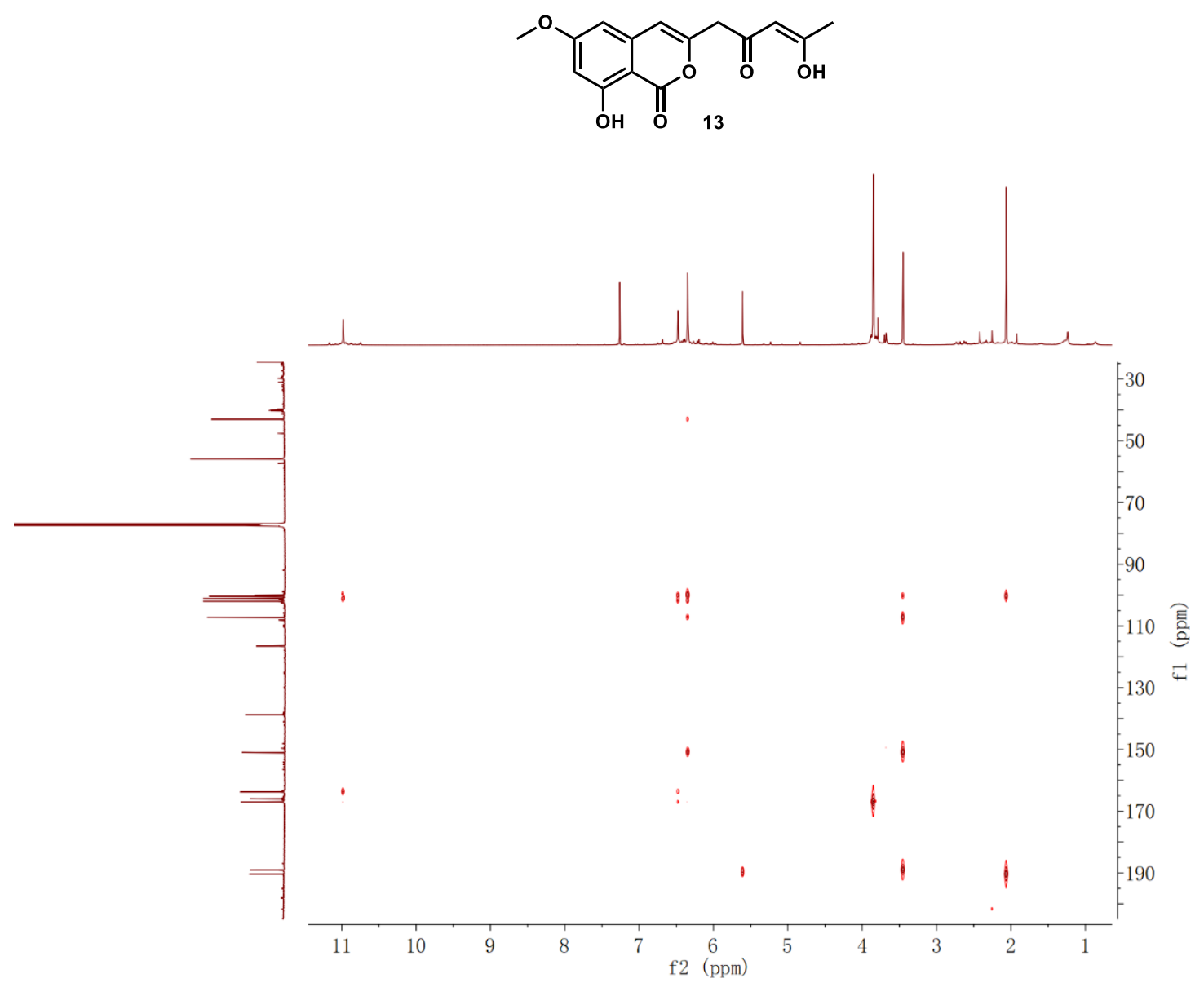

Figure S71. The HMBC spectrum of compound 13 in $\mathrm{CDCl}_{3}$. 

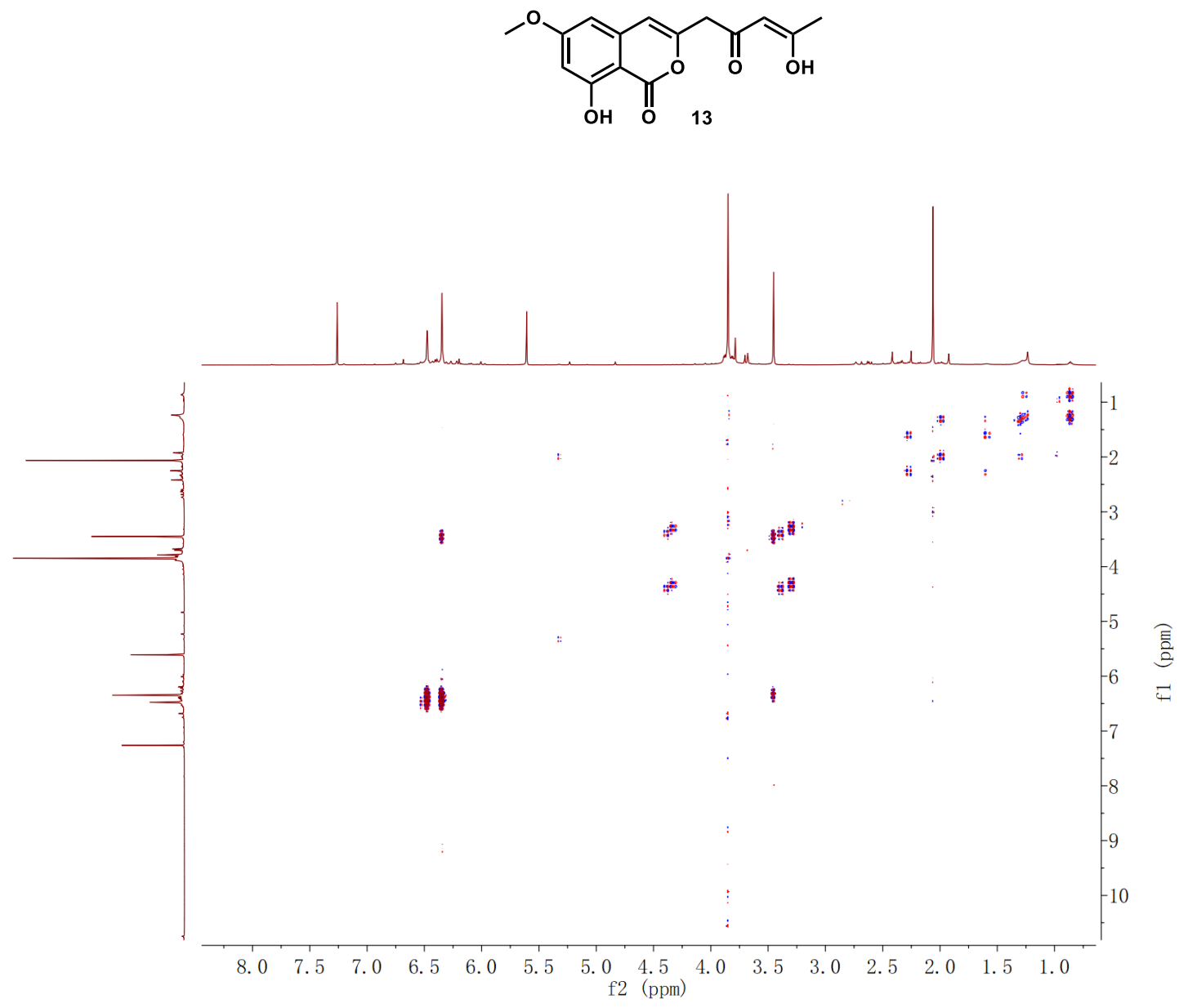

Figure S72. The ${ }^{1} \mathrm{H}-{ }^{1} \mathrm{H}$ COSY spectrum of compound 13 in $\mathrm{CDCl}_{3}$. 

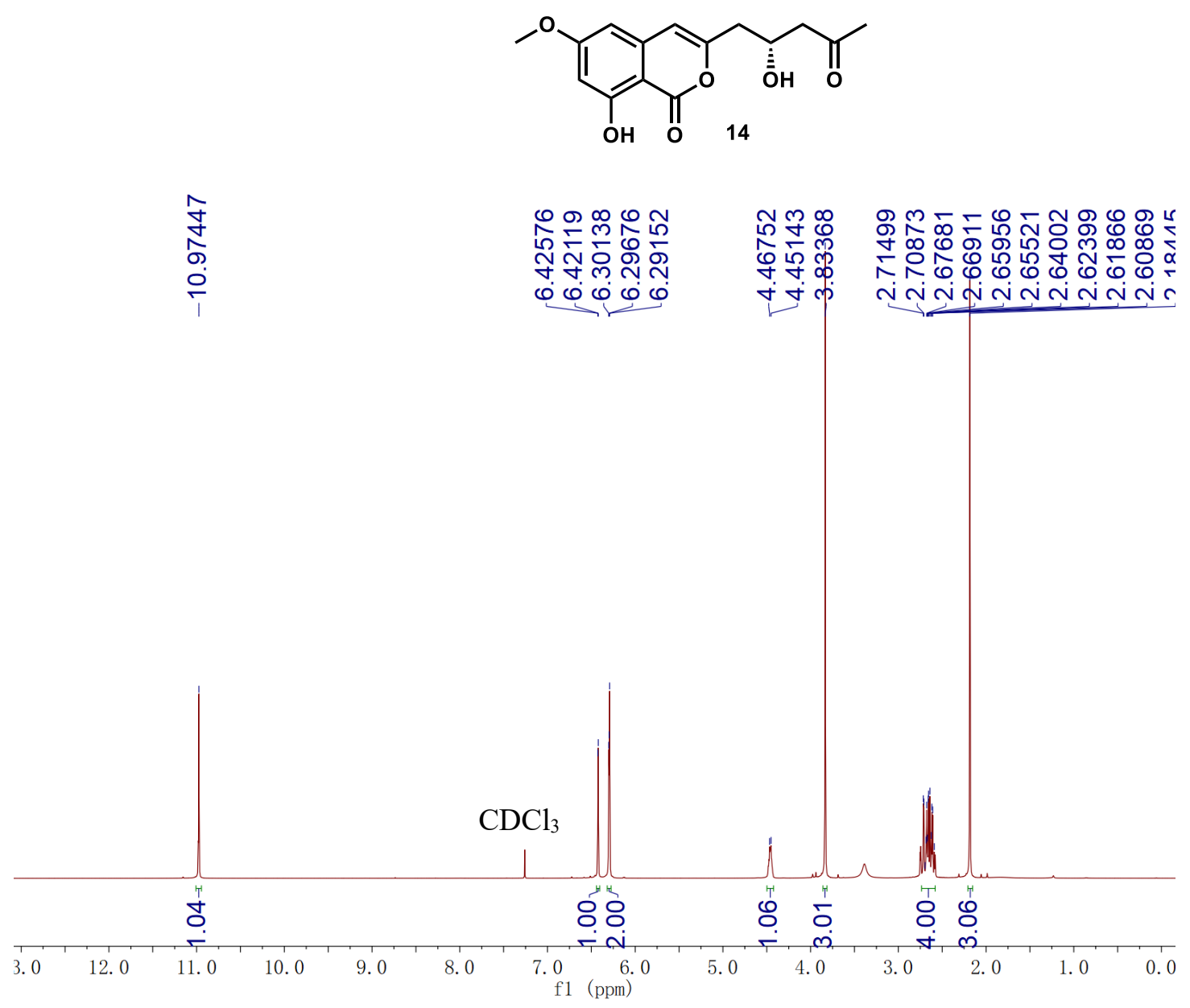

Figure S73. The ${ }^{1} \mathrm{H}$ NMR spectrum of compound 14 in $\mathrm{CDCl}_{3}$. 


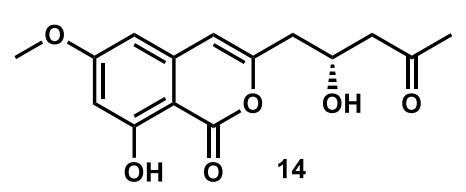

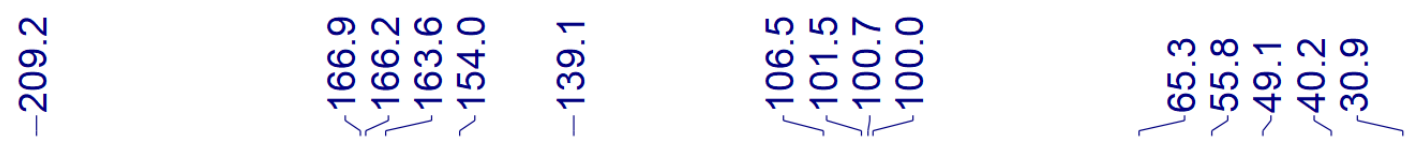
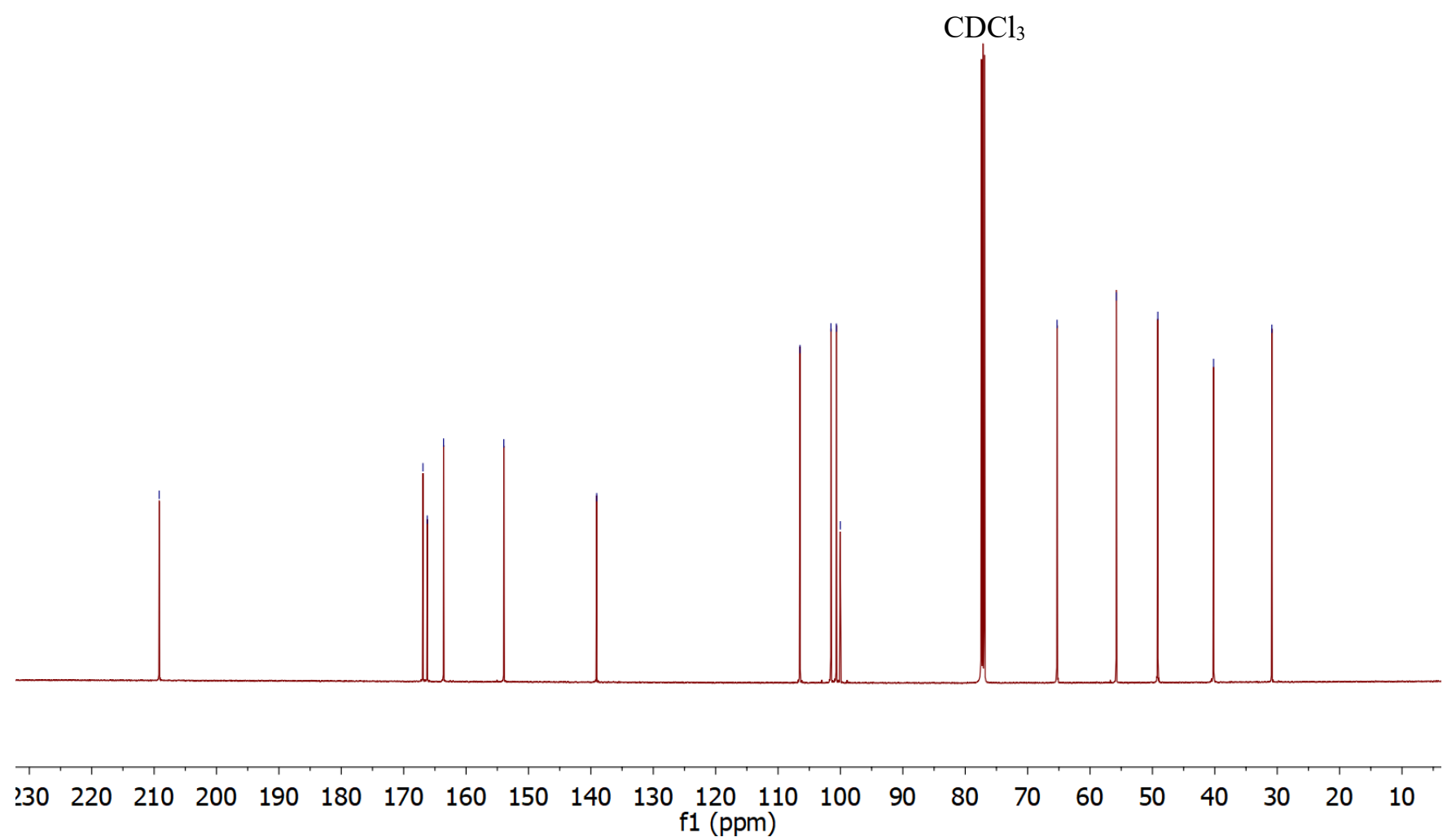

Figure S74. The ${ }^{13} \mathrm{C}$ NMR spectrum of compound 14 in $\mathrm{CDCl}_{3}$. 


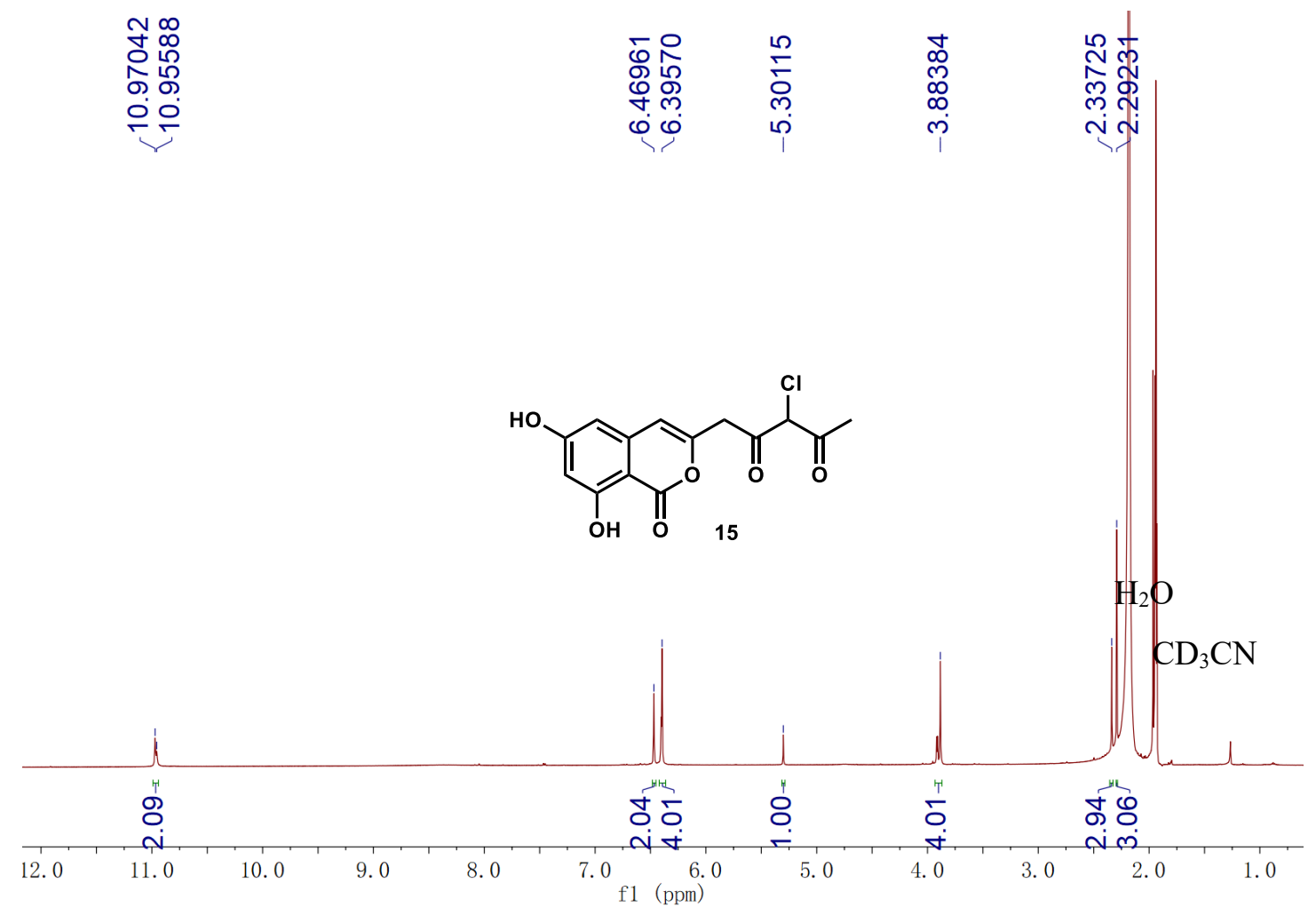

Figure S75. The ${ }^{1} \mathrm{H}$ NMR spectrum of compound 15 in acetonitrile- $d_{3}(500 \mathrm{MHz})$. 

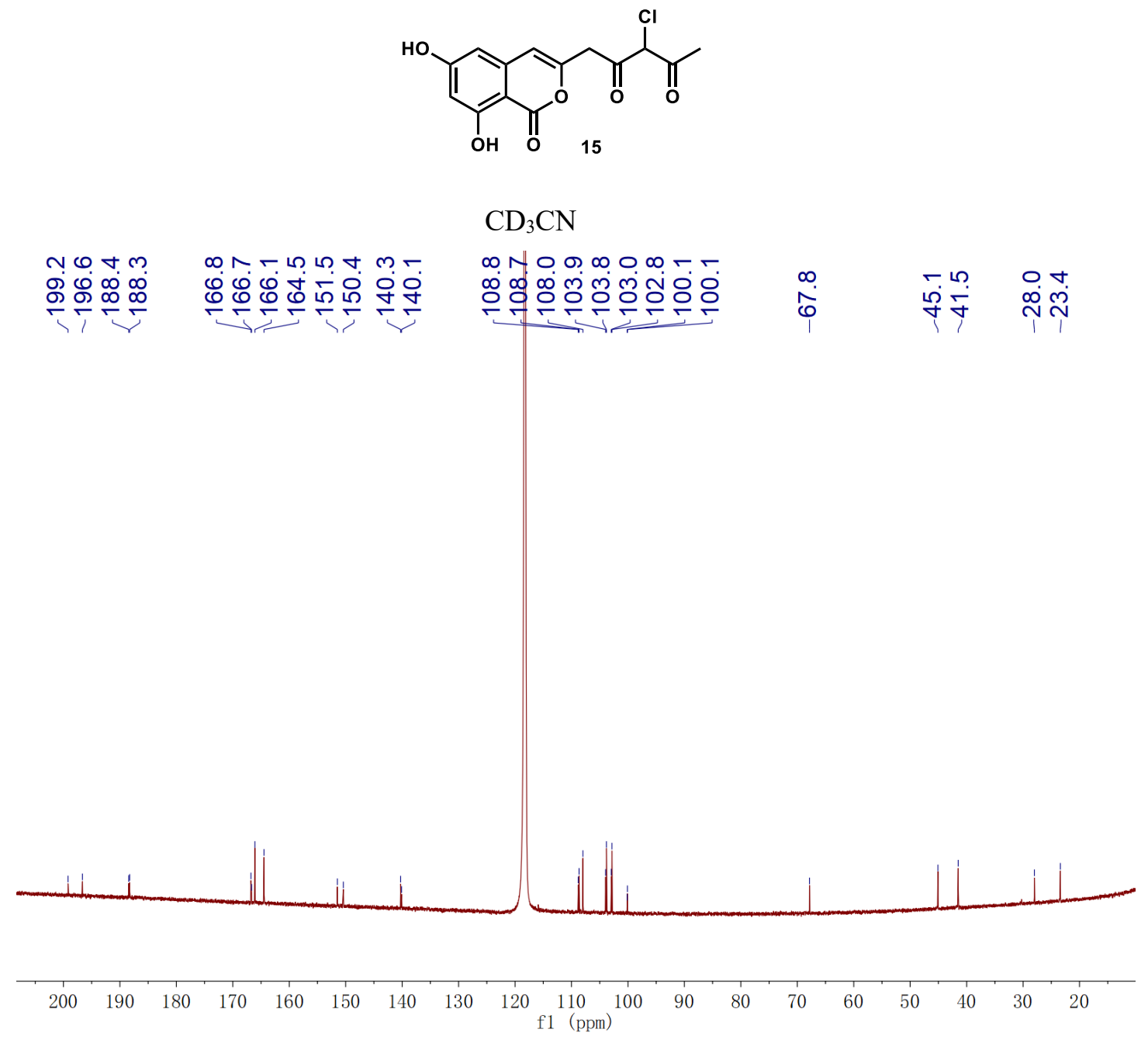

Figure S76. The ${ }^{13} \mathrm{C}$ NMR spectrum of compound 15 in acetonitrile- $d_{3}(125 \mathrm{MHz})$. 


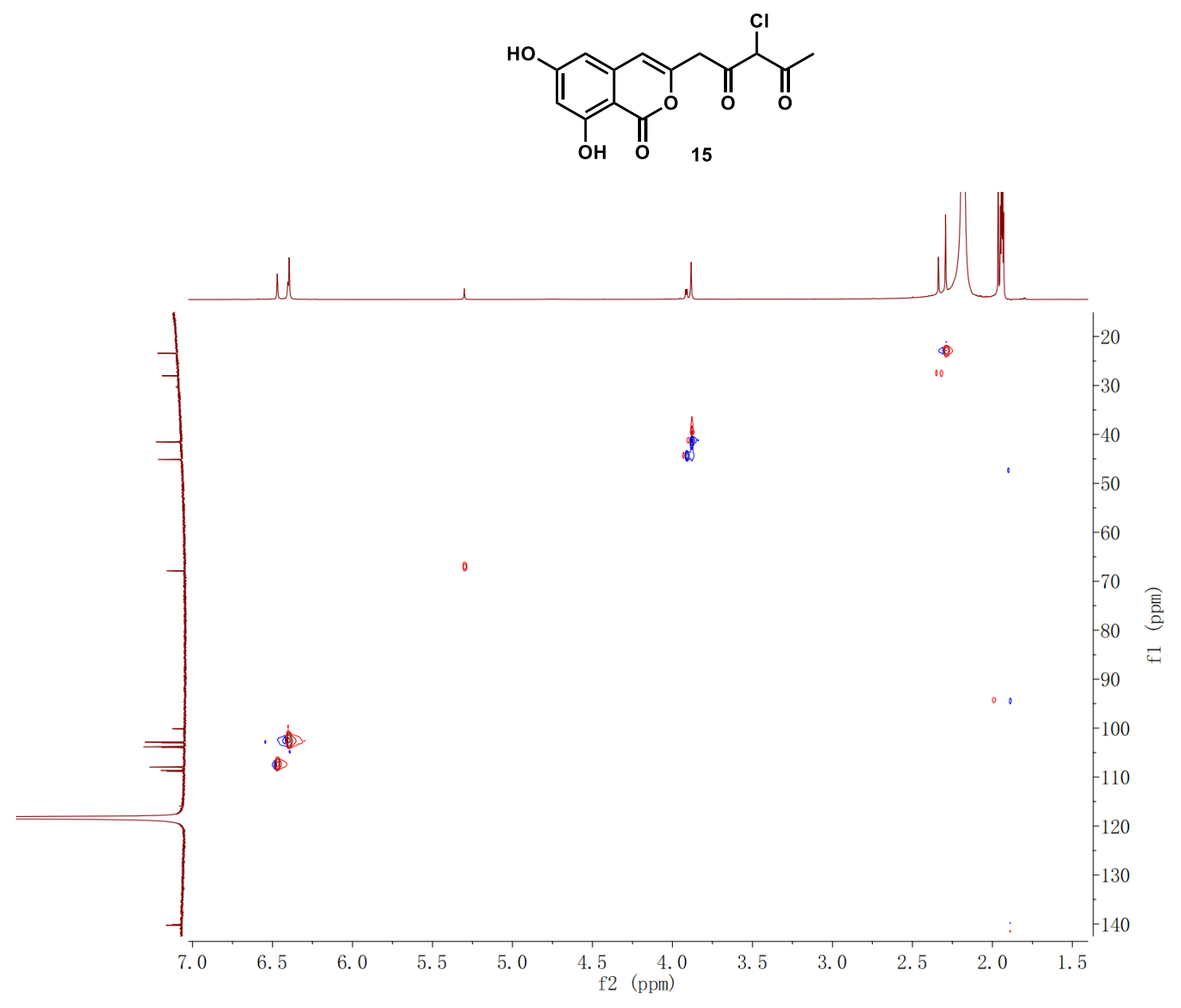

Figure S77. The HSQC spectrum of compound 15 in acetonitrile- $d_{3}$. 


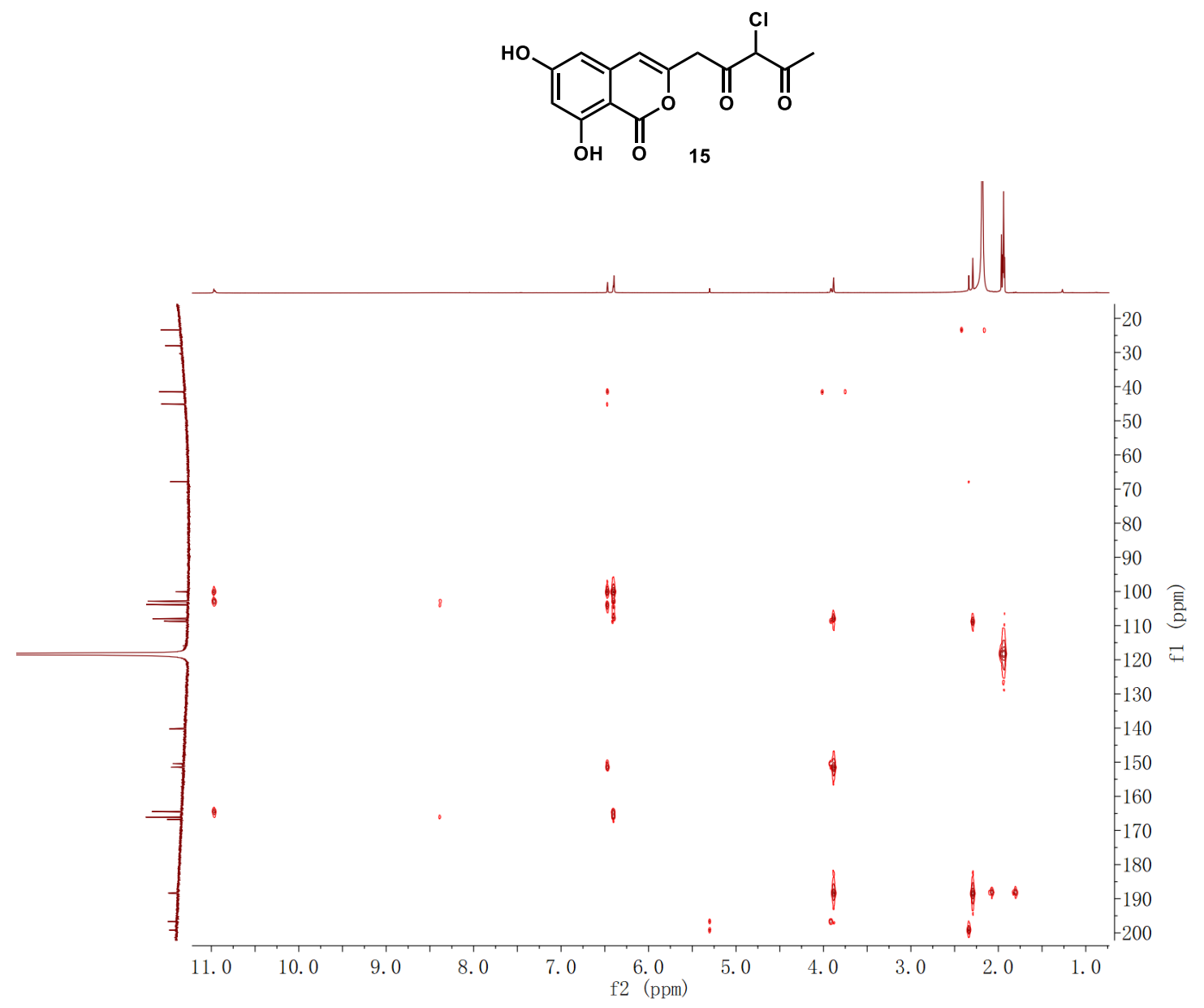

Figure S78. The HMBC spectrum of compound 15 in acetonitrile- $d_{3}$. 


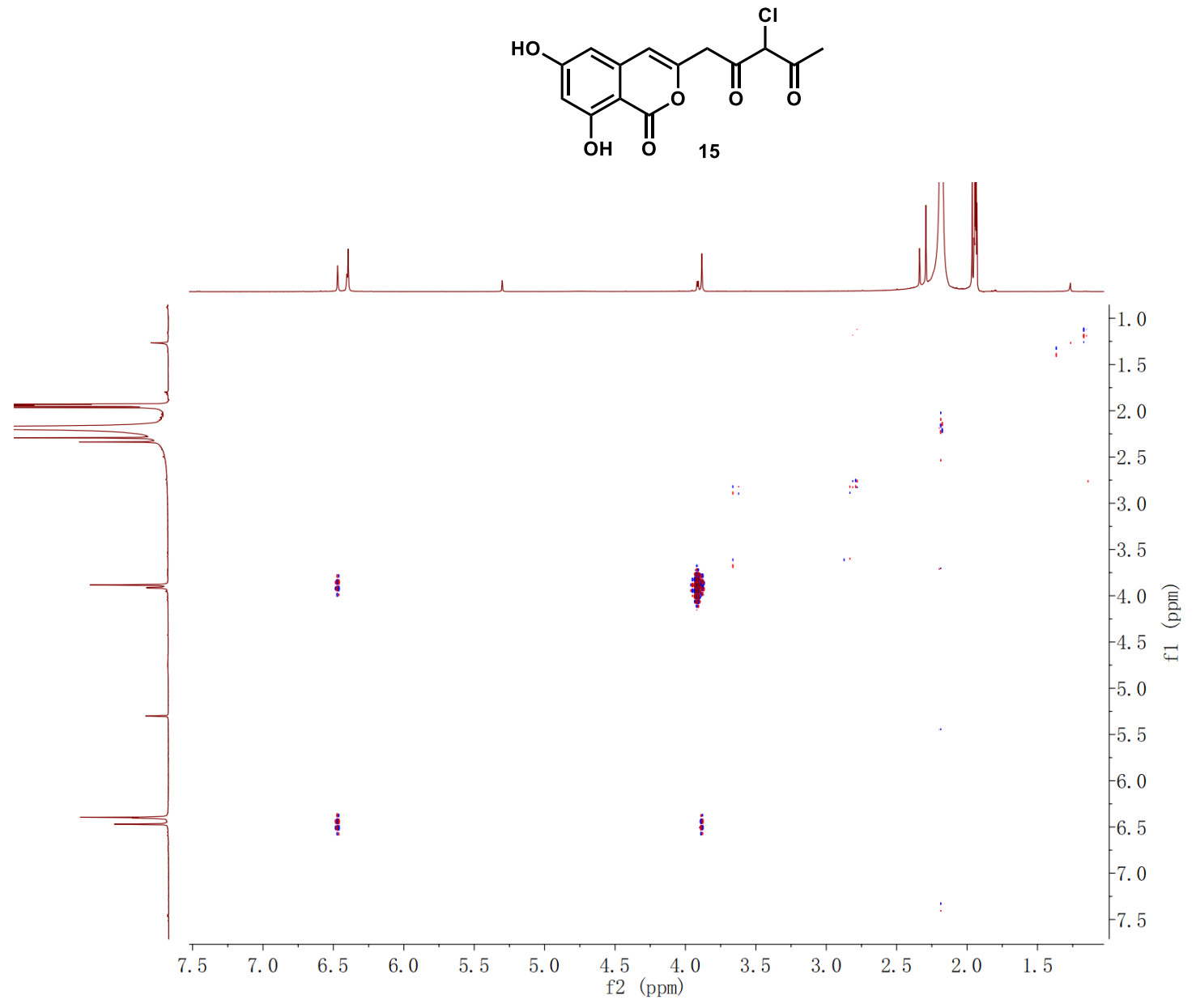

Figure S79. The ${ }^{1} \mathrm{H}-{ }^{1} \mathrm{H}$ COSY spectrum of compound $\mathbf{1 5}$ in acetonitrile- $d_{3}$. 

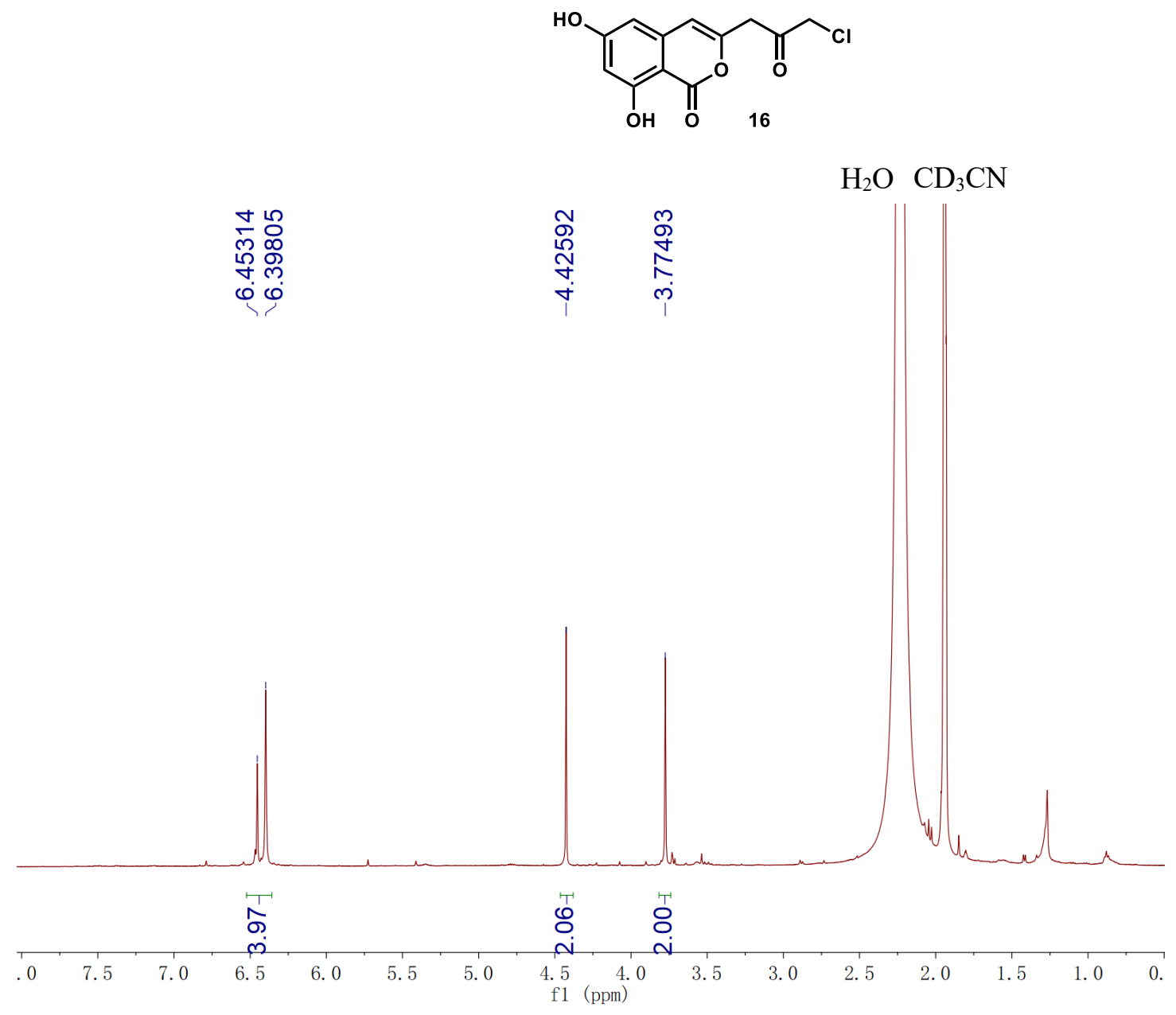

Figure S80. The ${ }^{1} \mathrm{H}$ NMR spectrum of compound 16 in acetonitrile- $d_{3}$. 

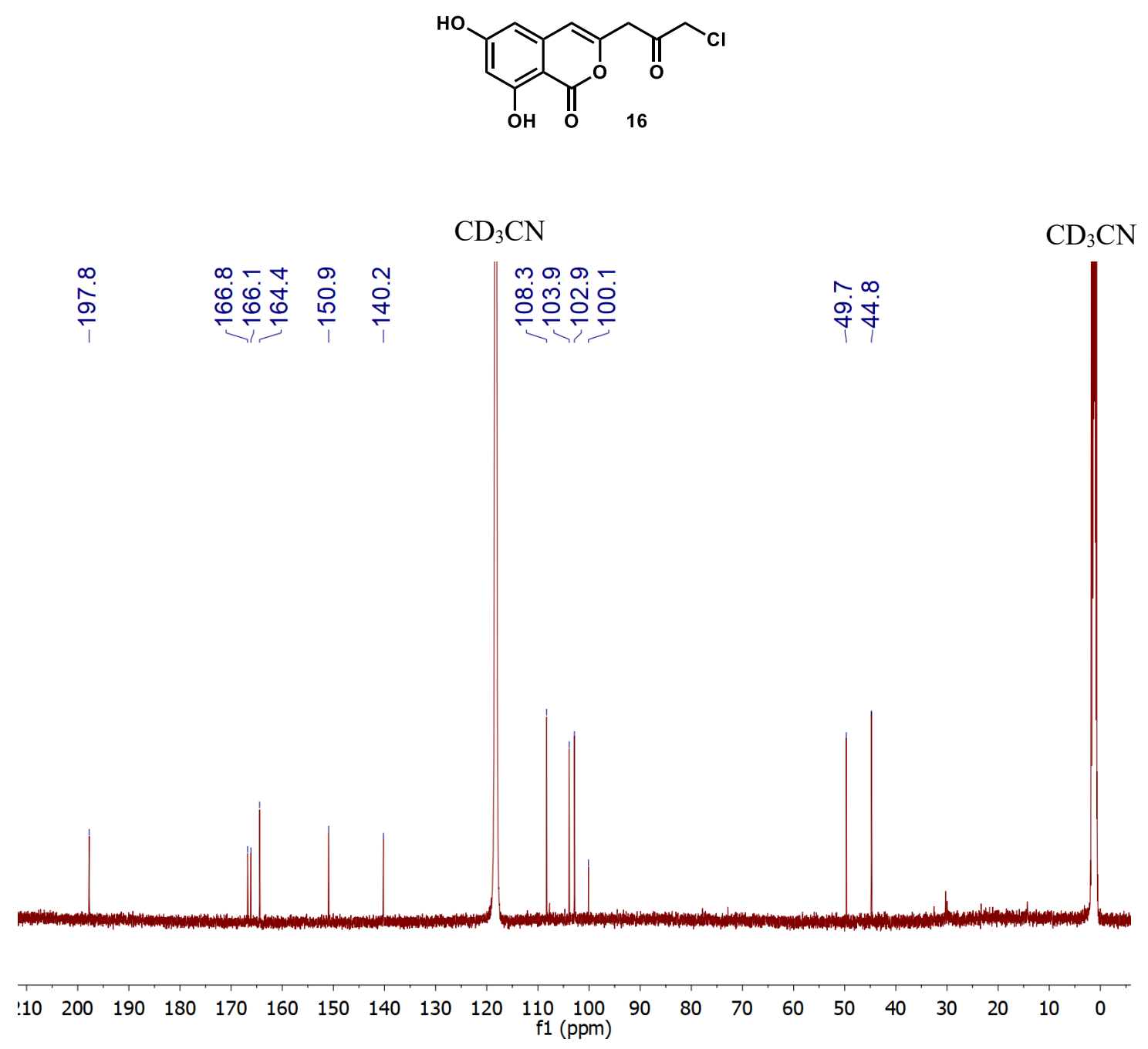

Figure S81. The ${ }^{13} \mathrm{C}$ NMR spectrum of compound $\mathbf{1 6}$ in acetonitrile- $d_{3}$. 


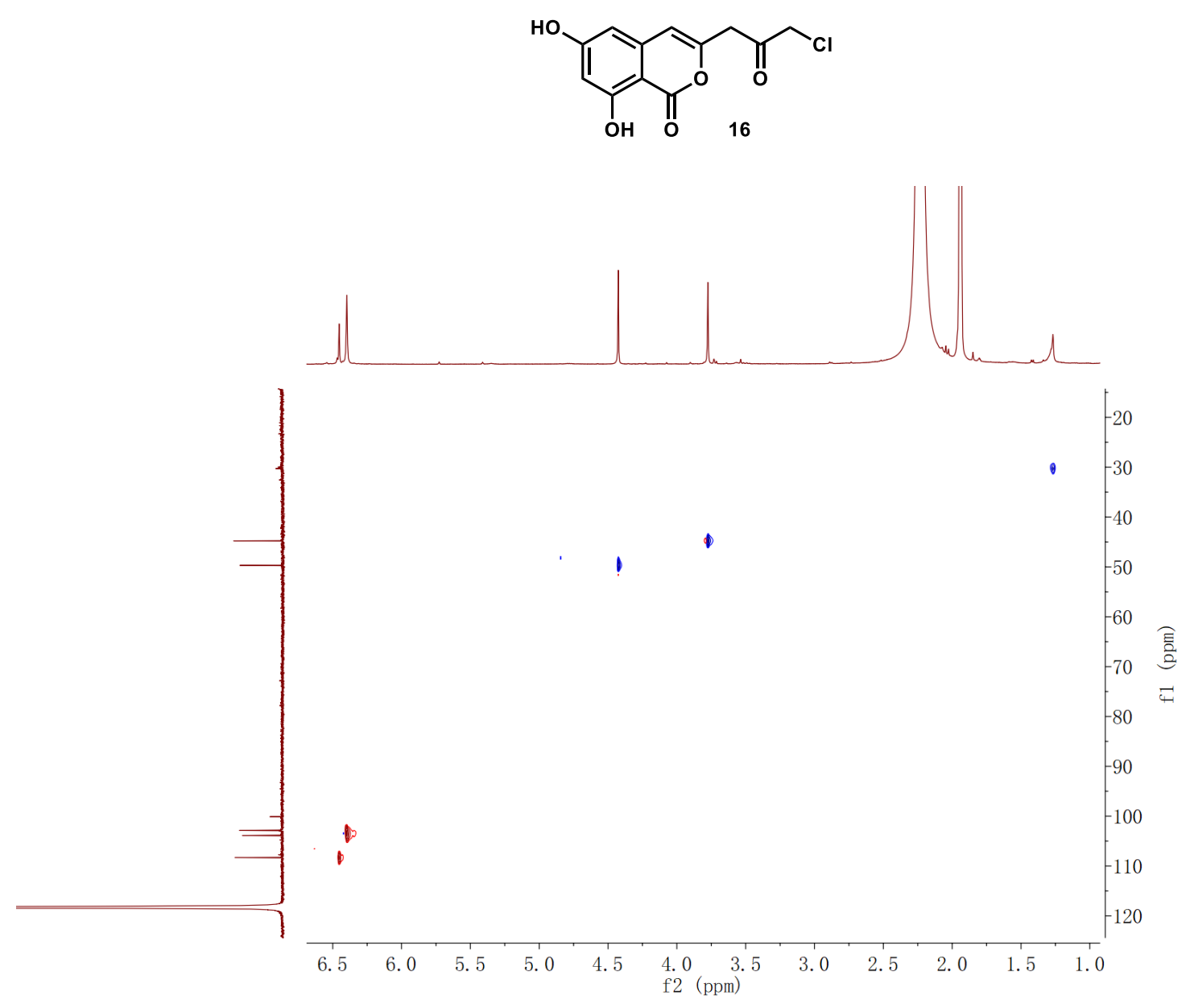

Figure S82. The HSQC spectrum of compound 16 in acetonitrile- $d_{3}$. 


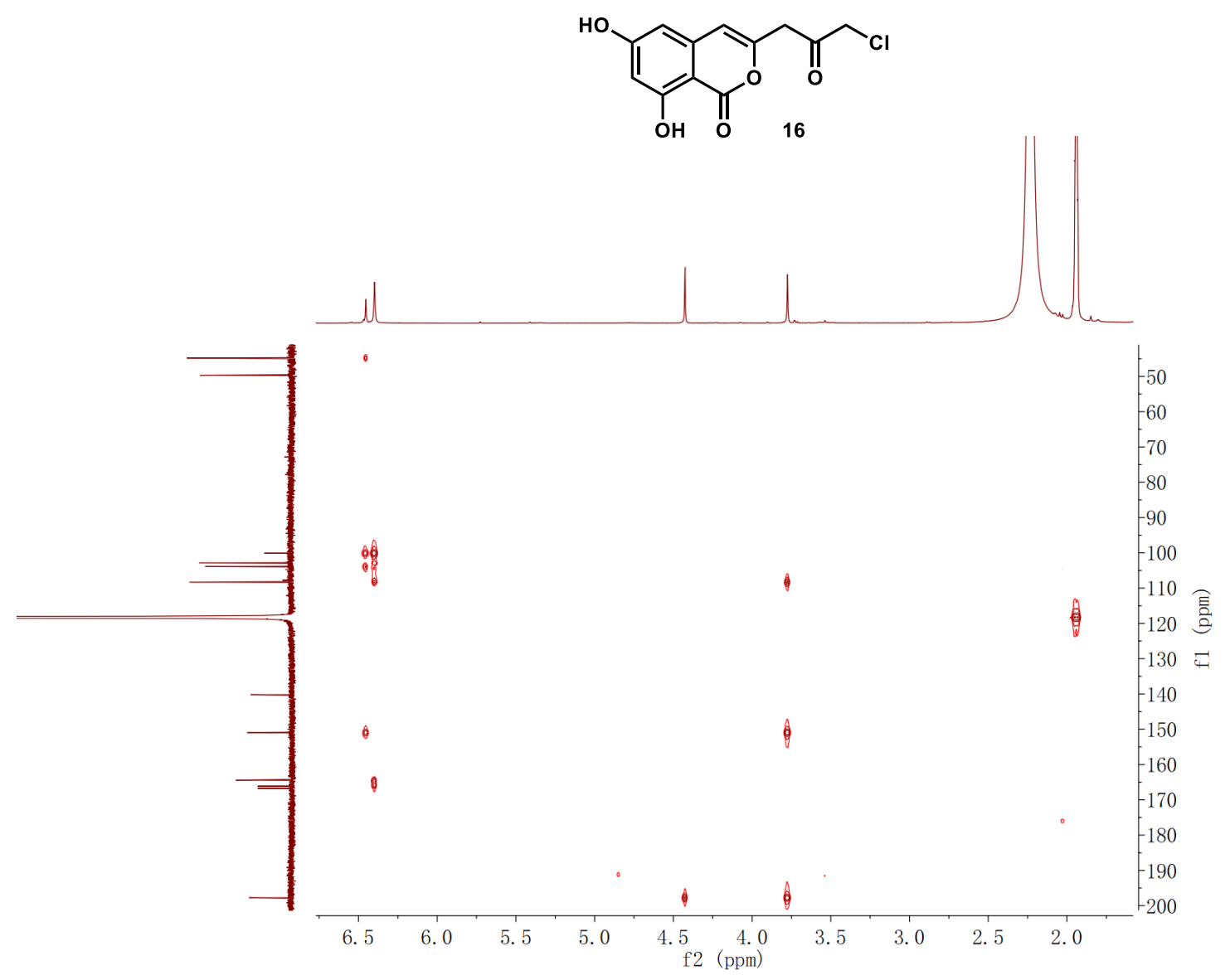

Figure S83. The HMBC spectrum of compound 16 in acetonitrile- $d_{3}$. 


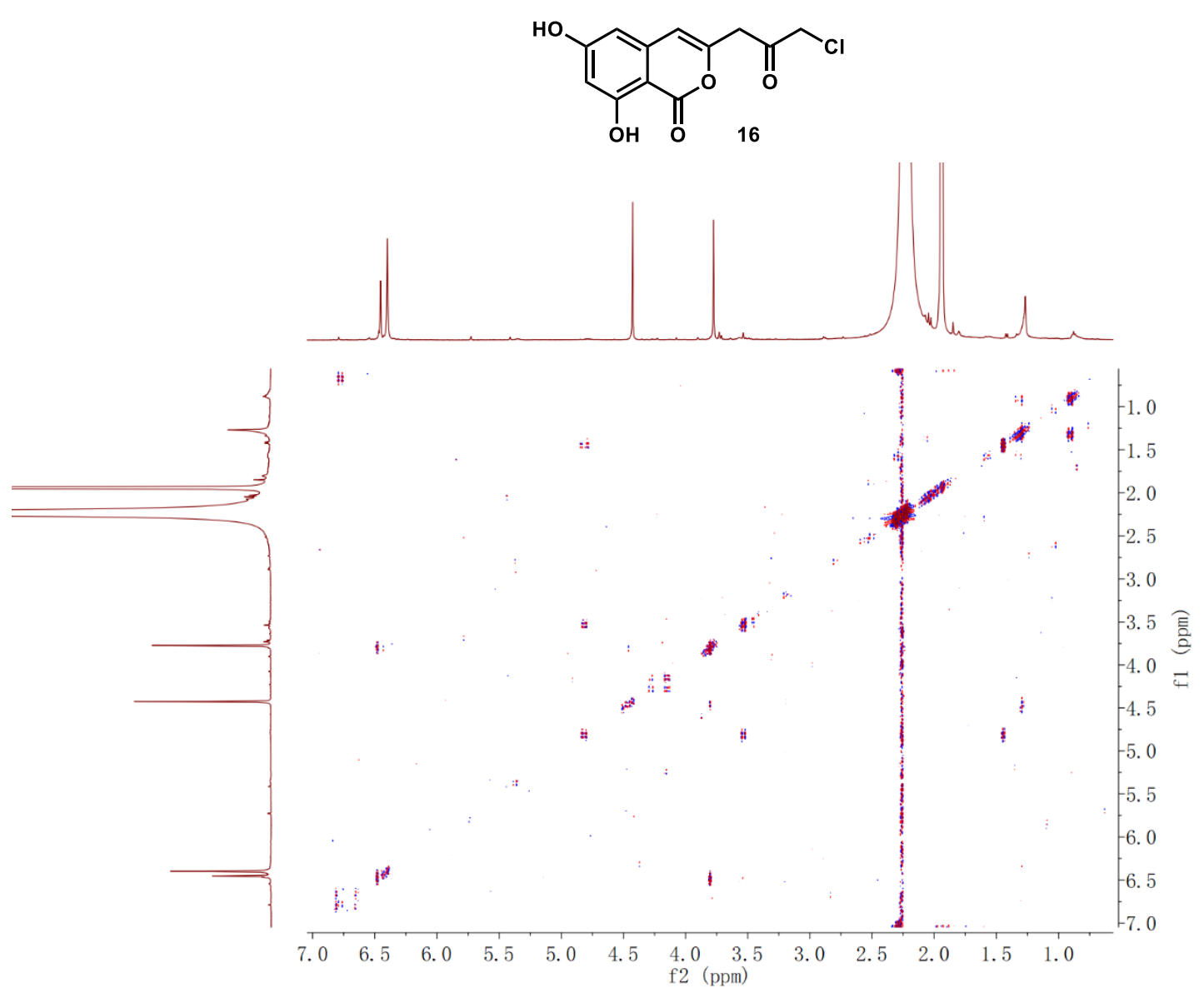

Figure S84. ${ }^{1} \mathrm{H}-{ }^{1} \mathrm{H}$ COSY spectrum of compound $\mathbf{1 6}$ in acetonitrile- $d_{3}$. 

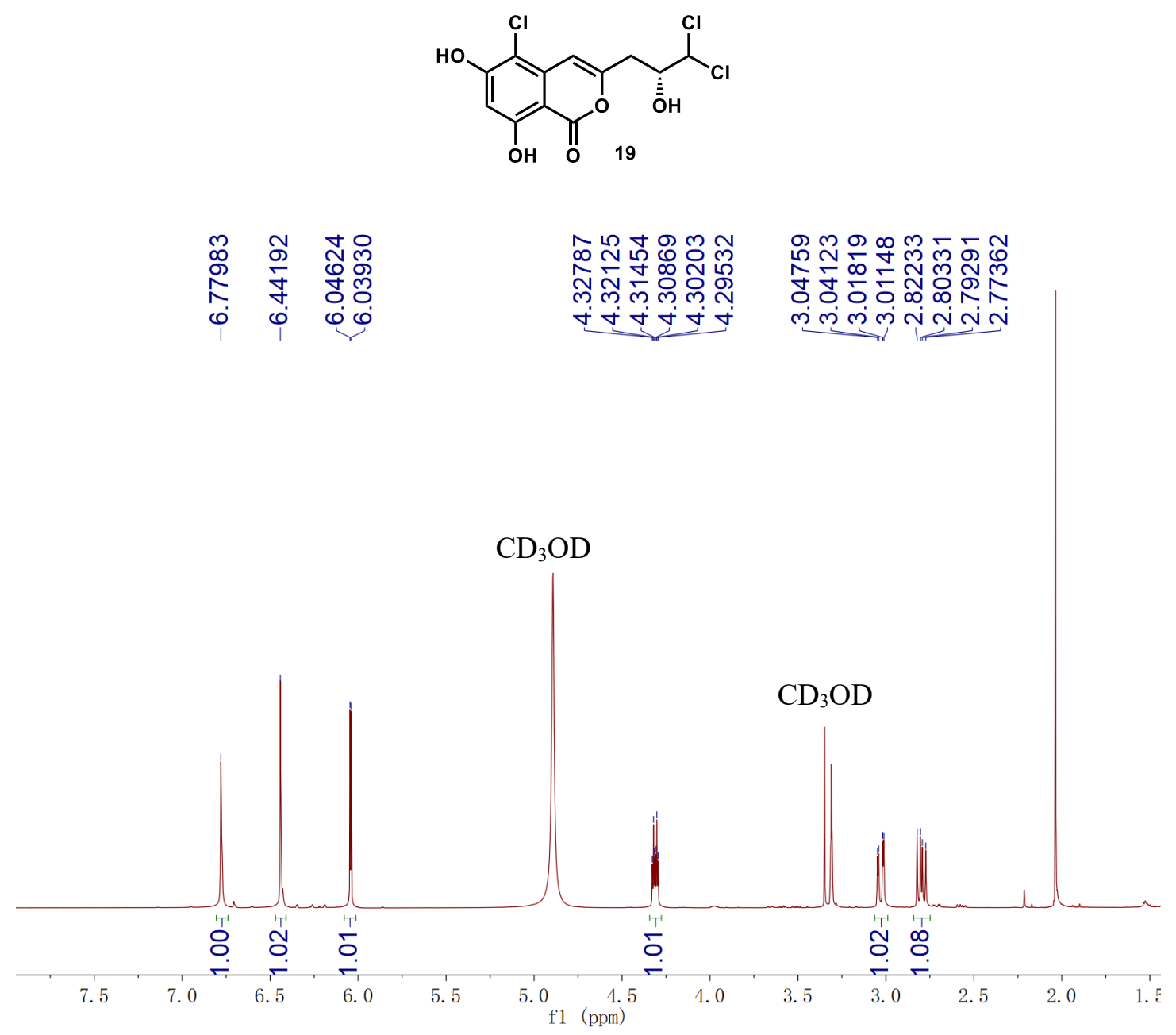

Figure S85. The ${ }^{1} \mathrm{H}$ NMR spectrum of compound 19 in $\mathrm{CD}_{3} \mathrm{OD}$. 

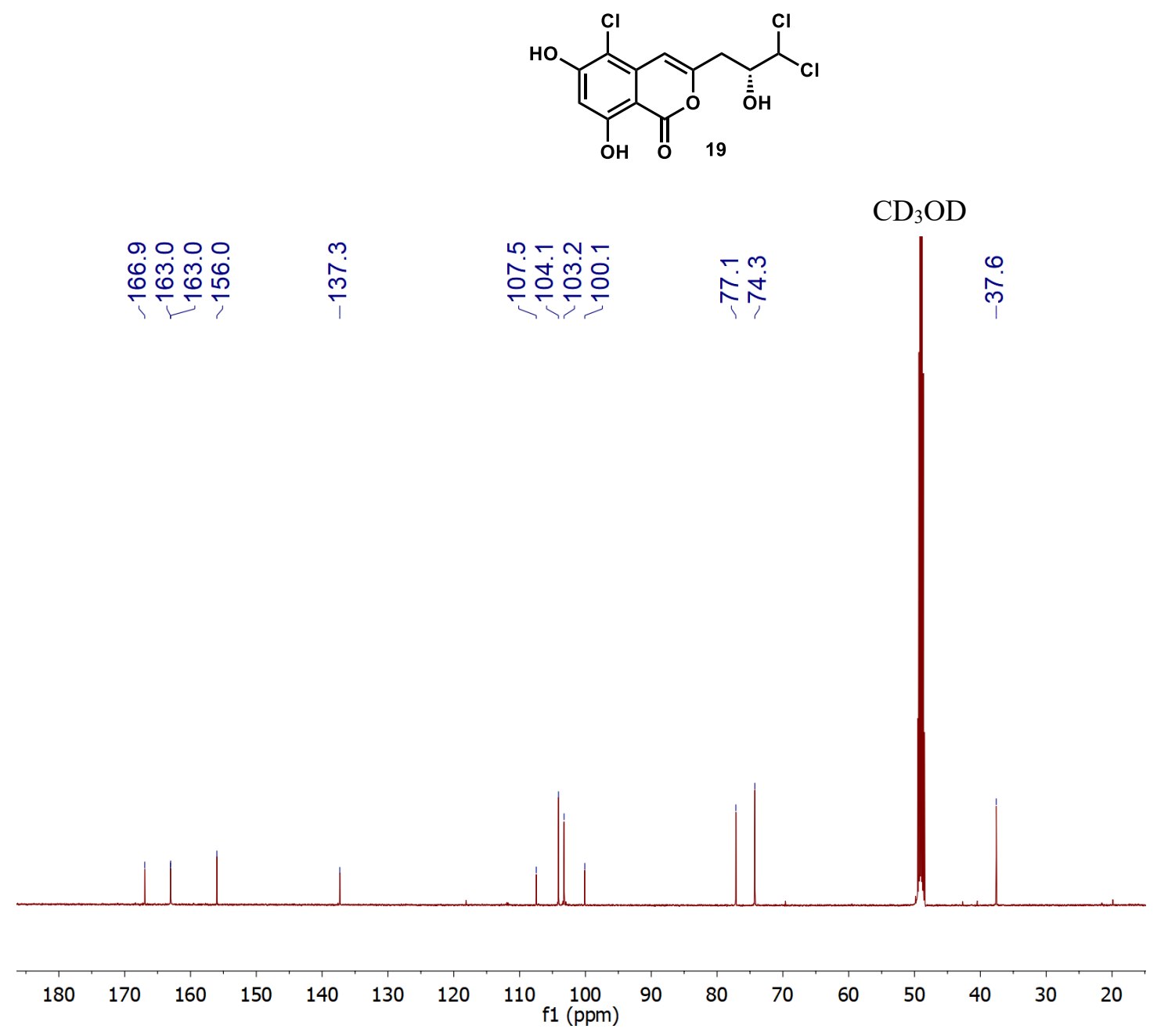

Figure S86. The ${ }^{13} \mathrm{C}$ NMR spectrum of compound 19 in $\mathrm{CD}_{3} \mathrm{OD}$. 


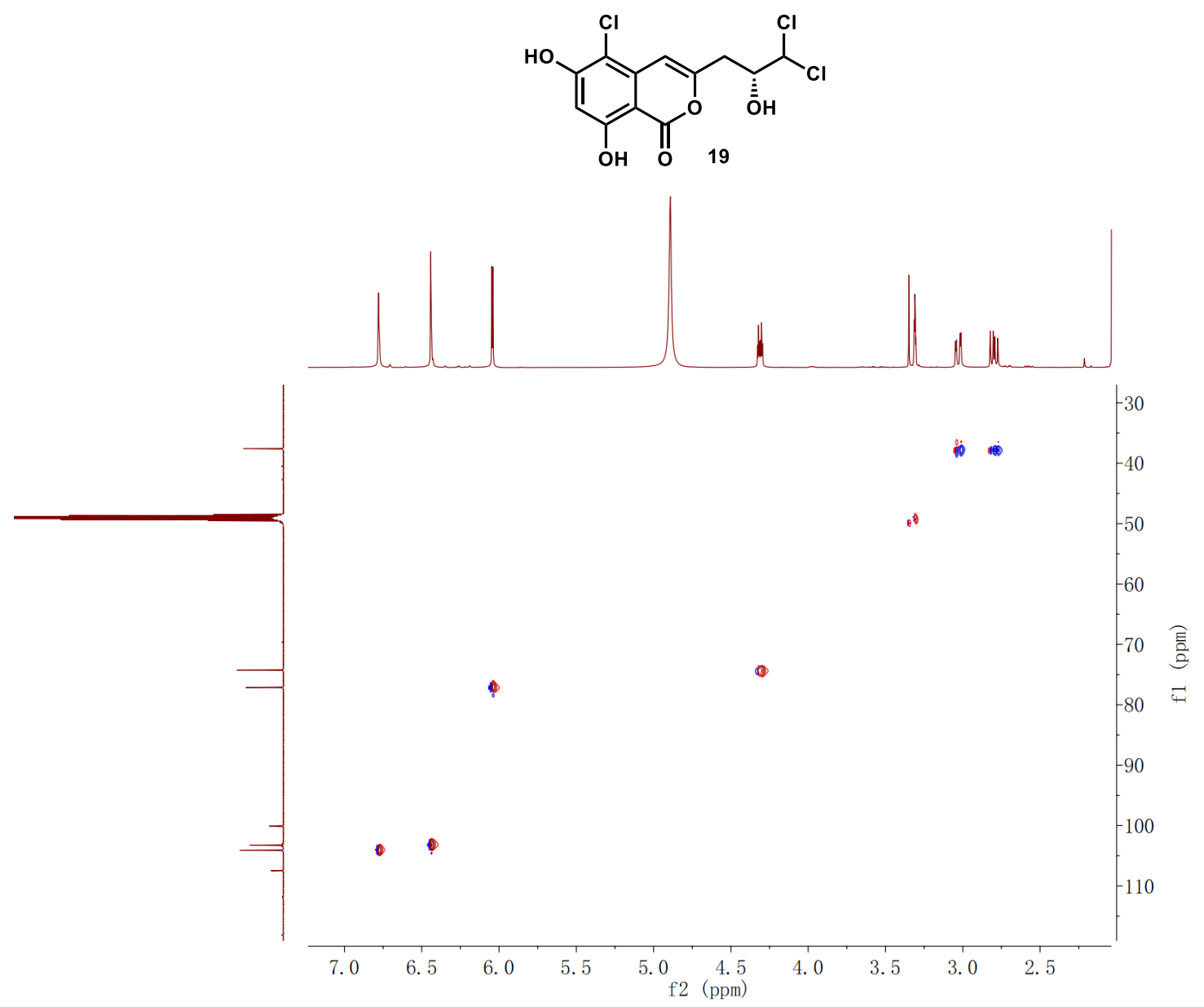

Figure S87. The HSQC spectrum of compound 19 in $\mathrm{CD}_{3} \mathrm{OD}$. 


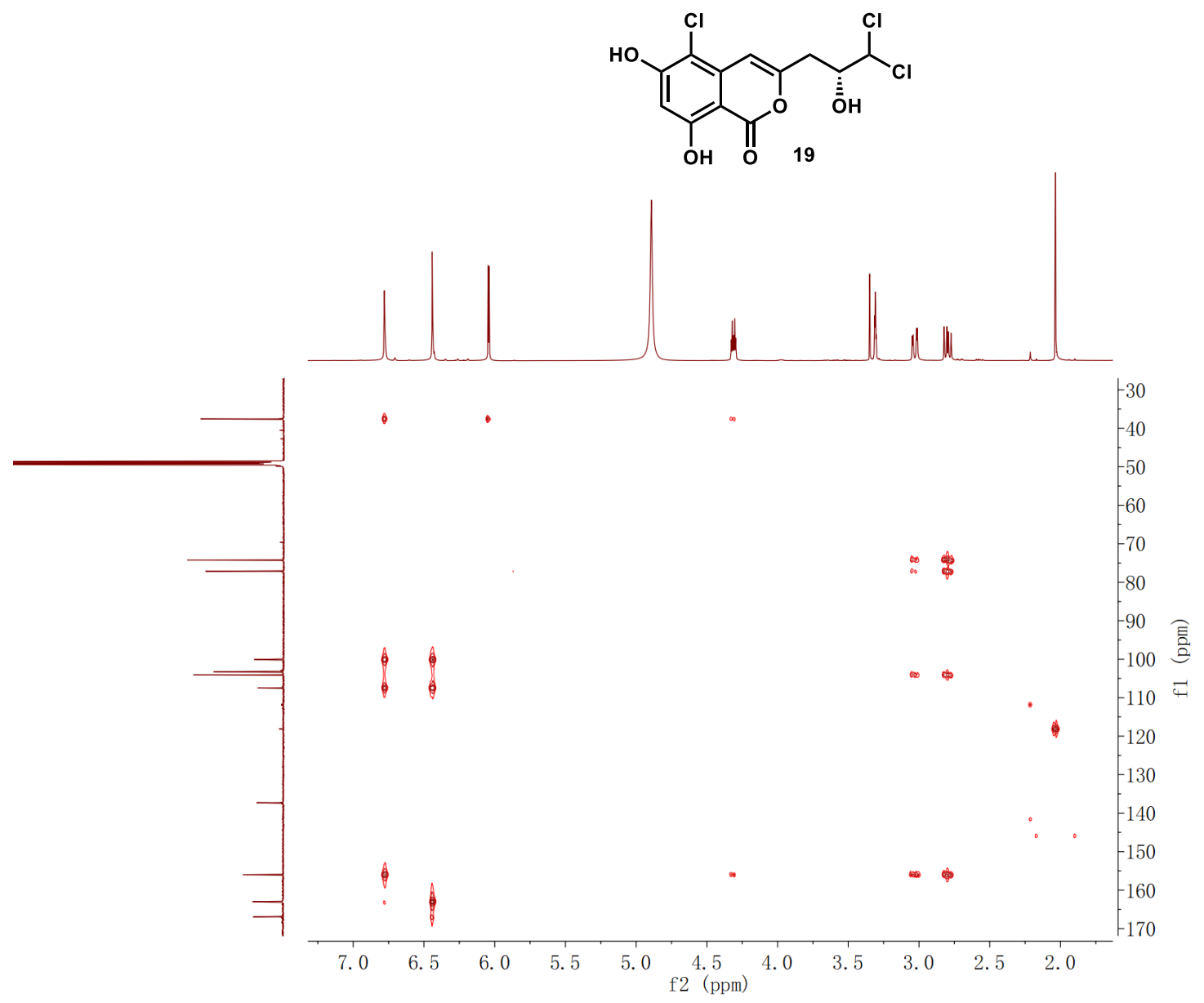

Figure S88. The HMBC spectrum of compound 19 in $\mathrm{CD}_{3} \mathrm{OD}$. 


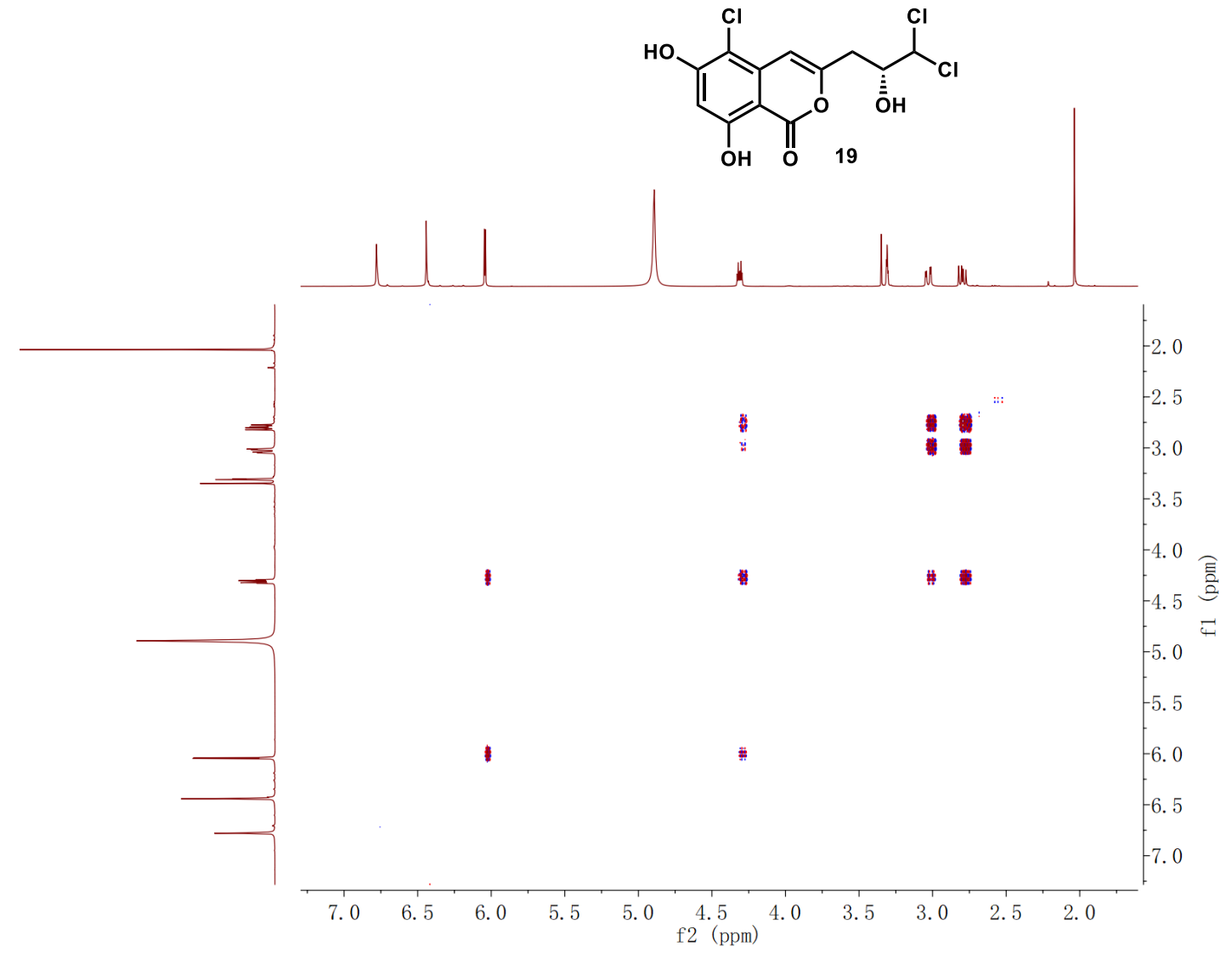

Figure S89. The ${ }^{1} \mathrm{H}-{ }^{1} \mathrm{H}$ COSY spectrum of compound 19 in $\mathrm{CD}_{3} \mathrm{OD}$. 

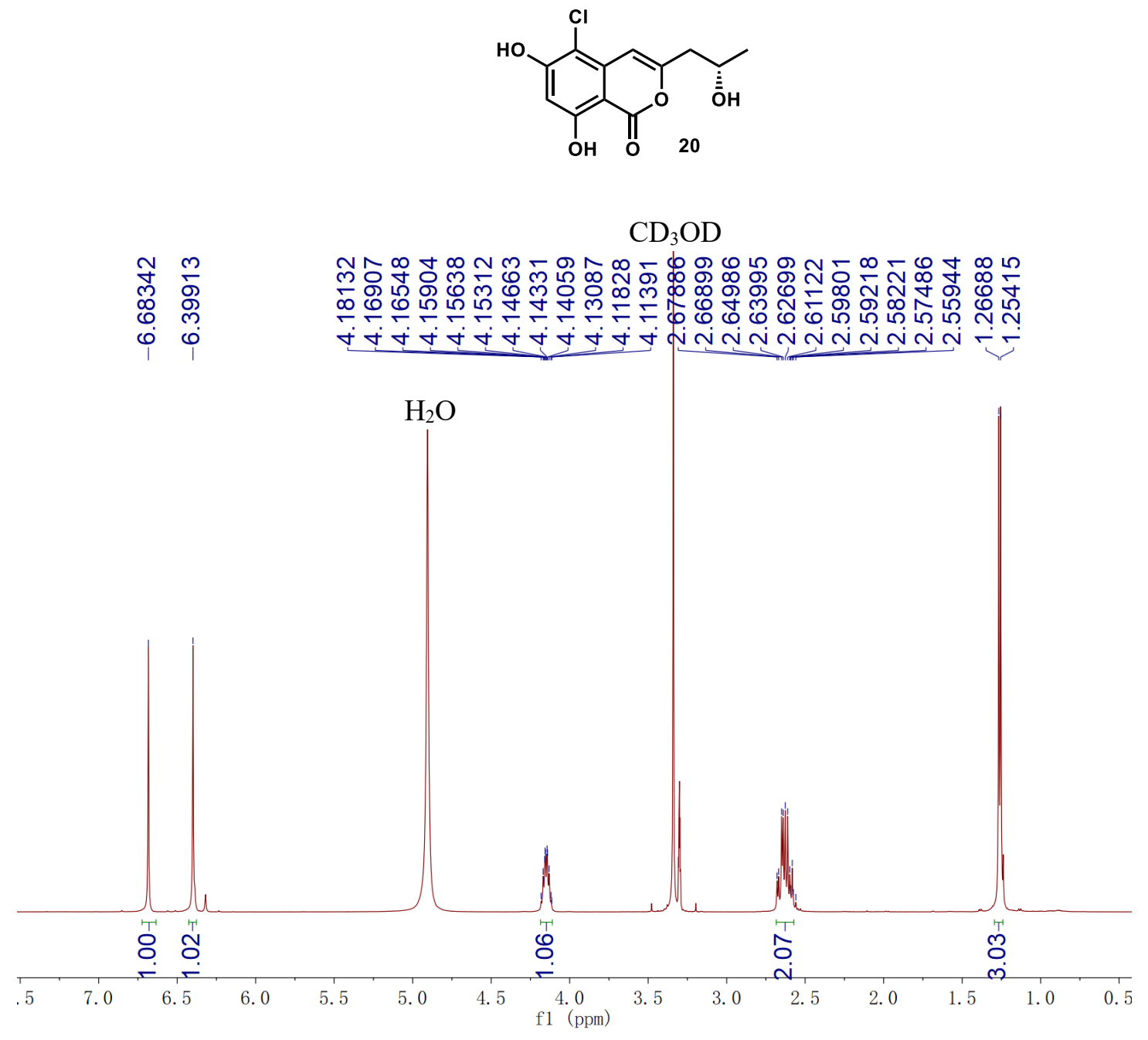

Figure S90. The ${ }^{1} \mathrm{H}$ NMR spectrum of compound 20 in $\mathrm{CD}_{3} \mathrm{OD}$. 

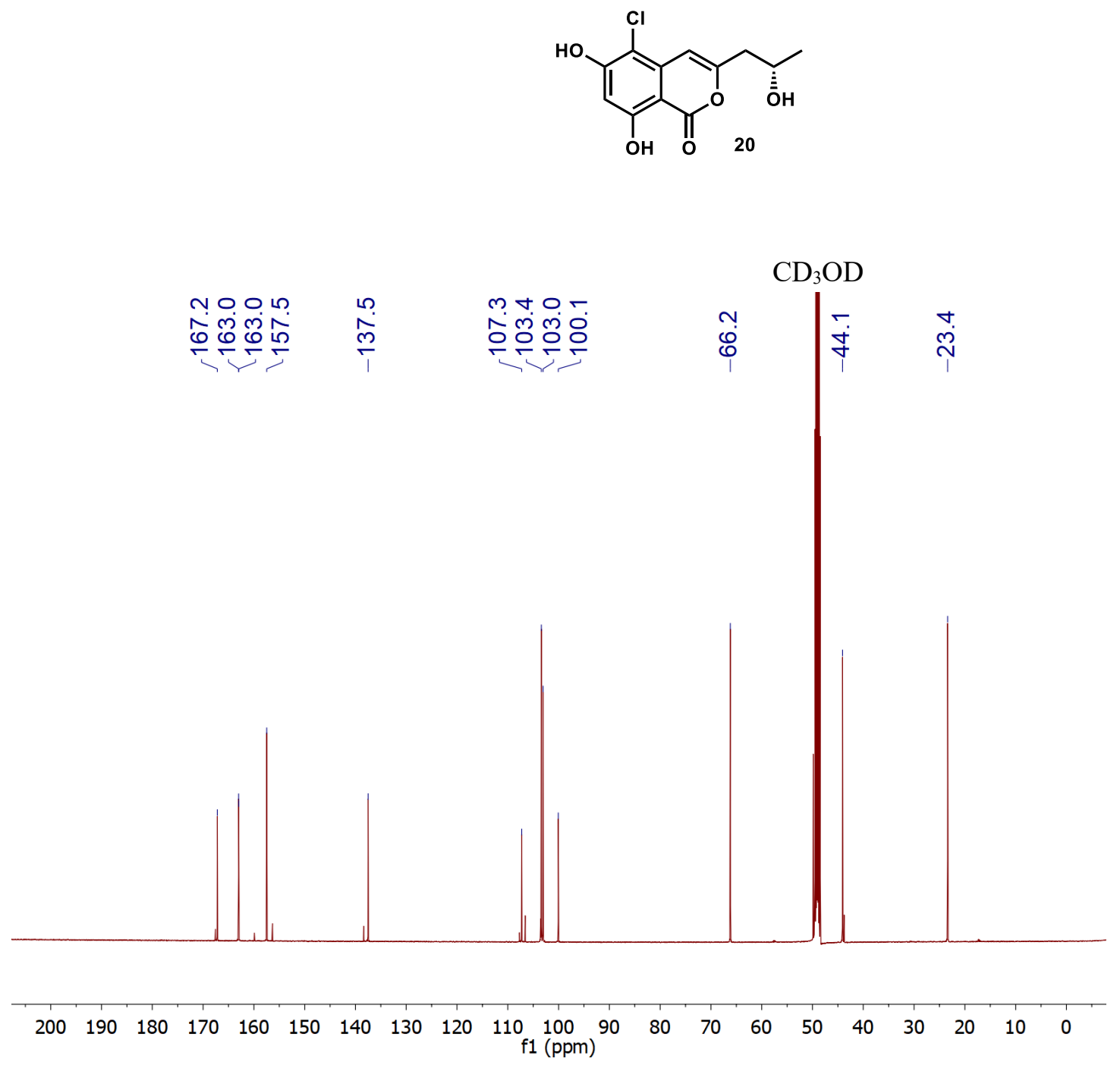

Figure S91. The ${ }^{13} \mathrm{C}$ NMR spectrum of compound 20 in $\mathrm{CD}_{3} \mathrm{OD}$. 

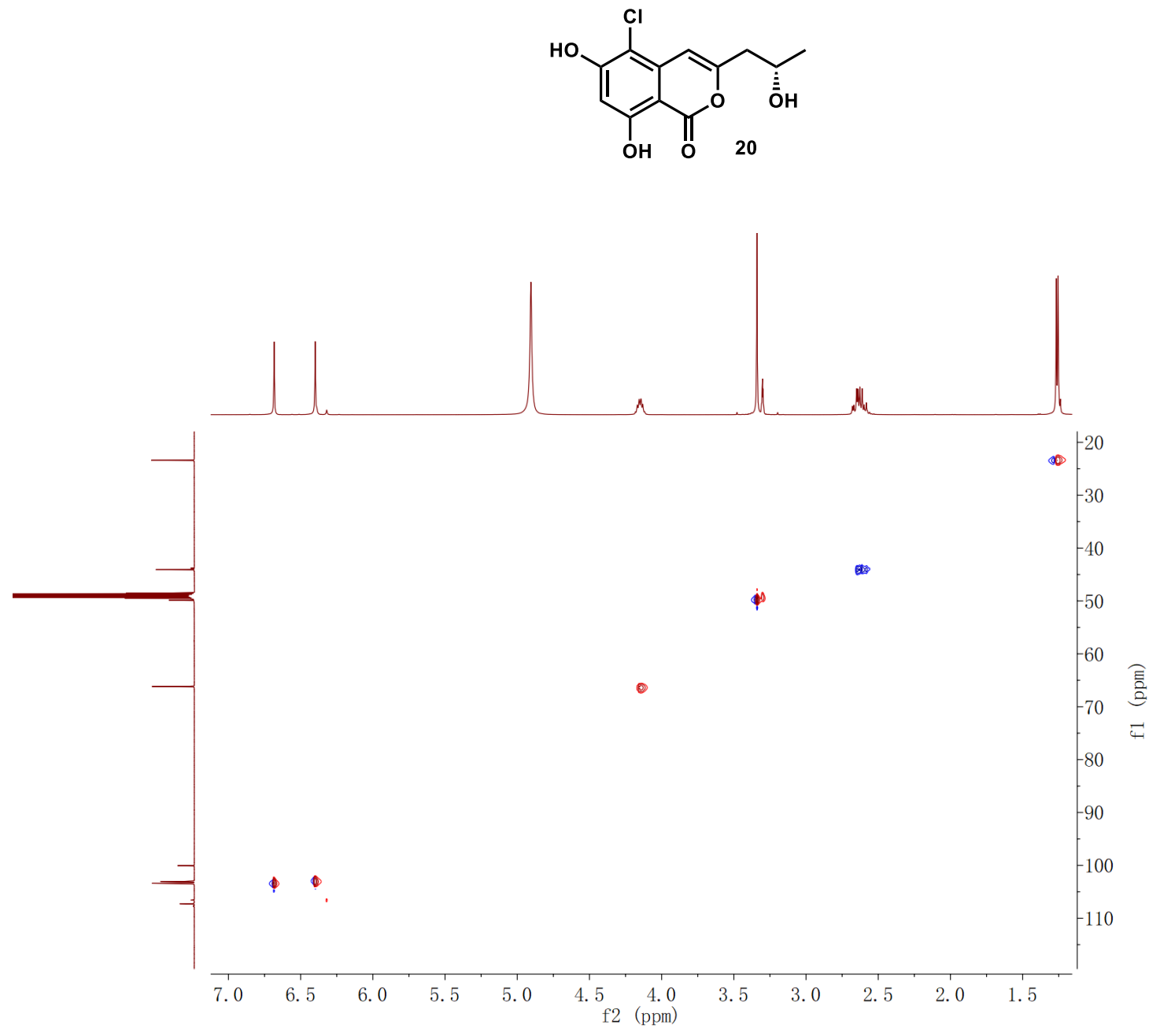

Figure S92. The HSQC spectrum of compound $\mathbf{2 0}$ in $\mathrm{CD}_{3} \mathrm{OD}$. 

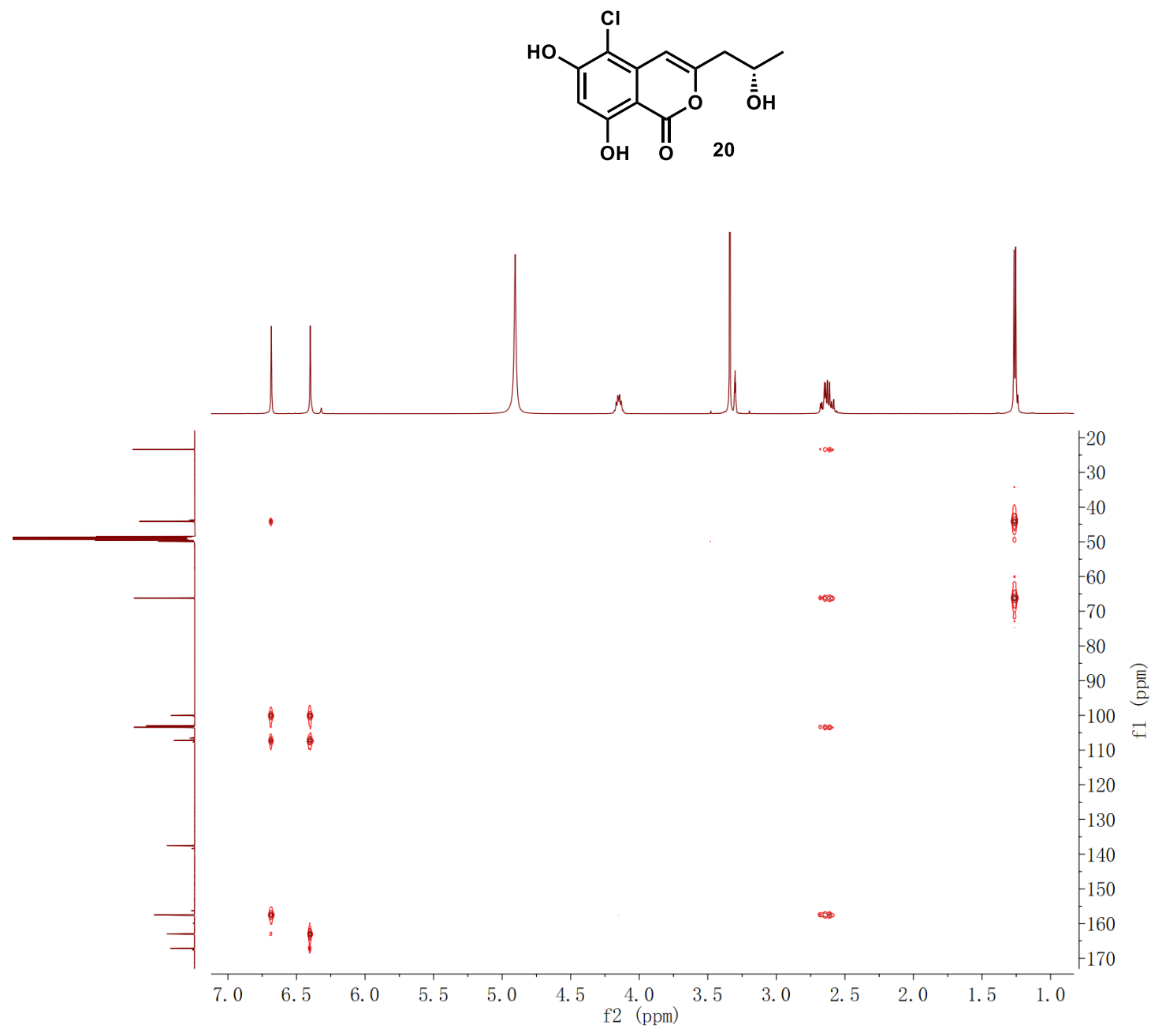

Figure S93. The HMBC spectrum of compound 20 in $\mathrm{CD}_{3} \mathrm{OD}$. 

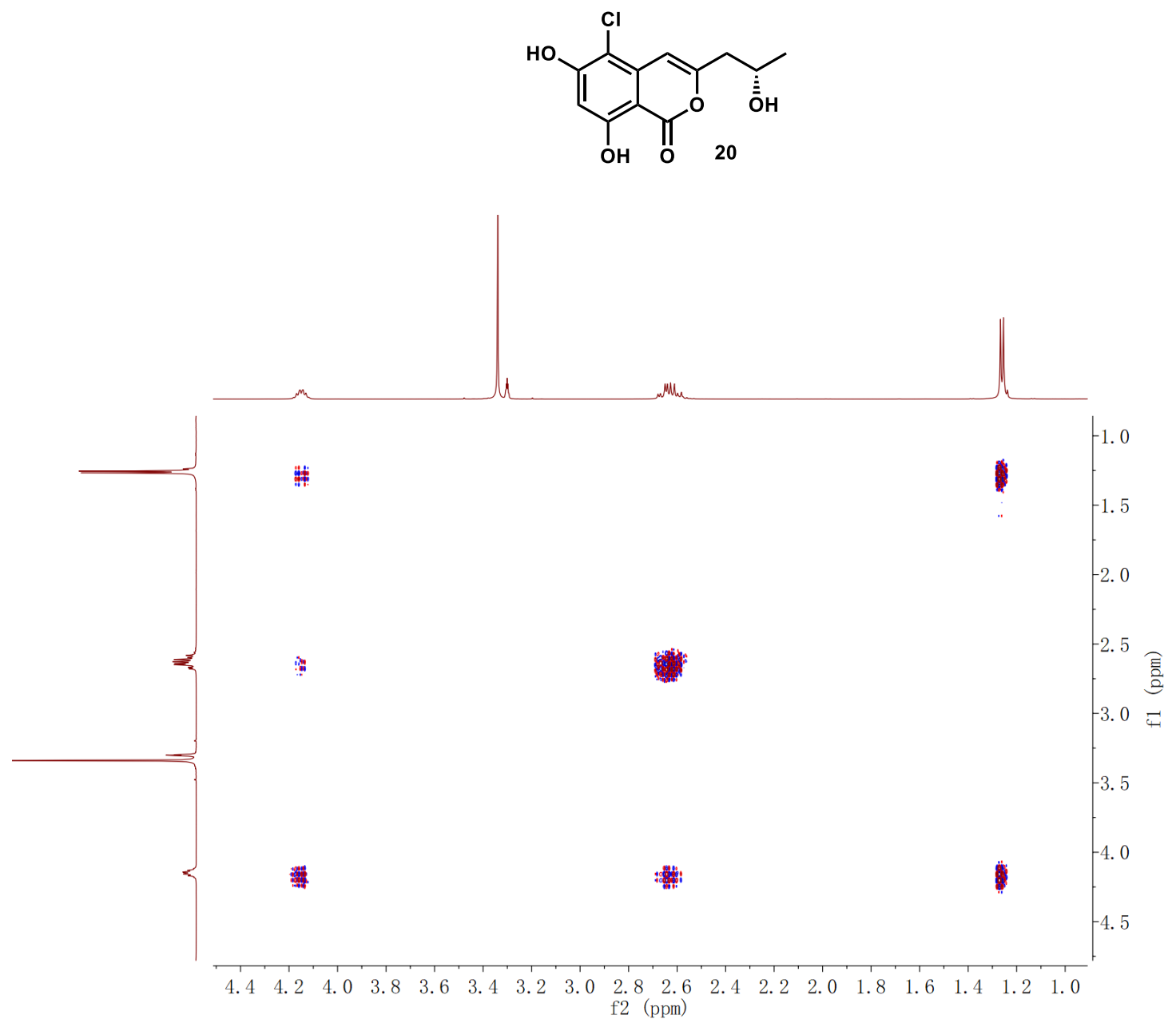

Figure S94. The ${ }^{1} \mathrm{H}-{ }^{1} \mathrm{H}$ COSY spectrum of compound 20 in $\mathrm{CD}_{3} \mathrm{OD}$. 

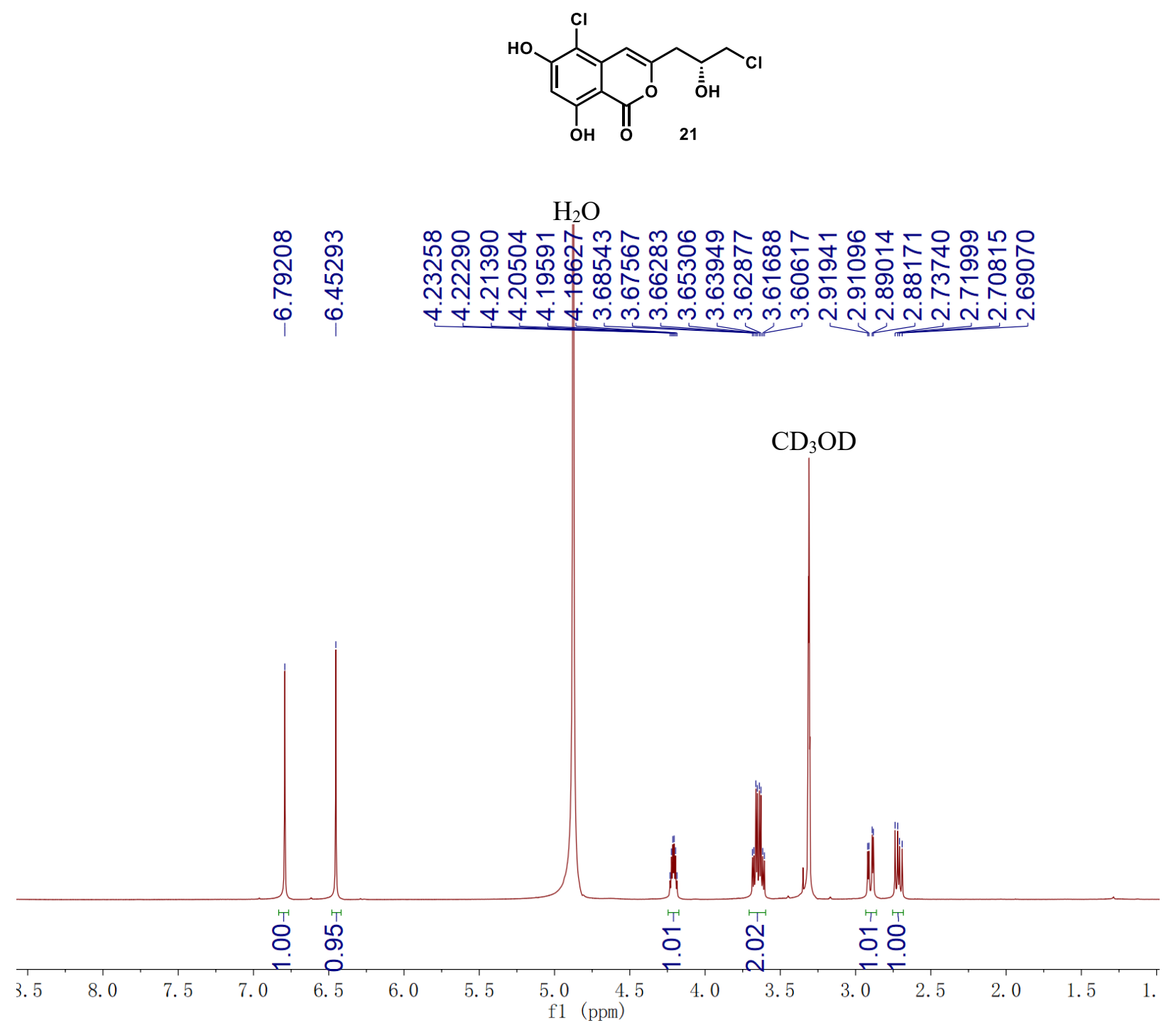

Figure S95. The ${ }^{1} \mathrm{H}$ NMR spectrum of compound 21 in $\mathrm{CD}_{3} \mathrm{OD}$. 

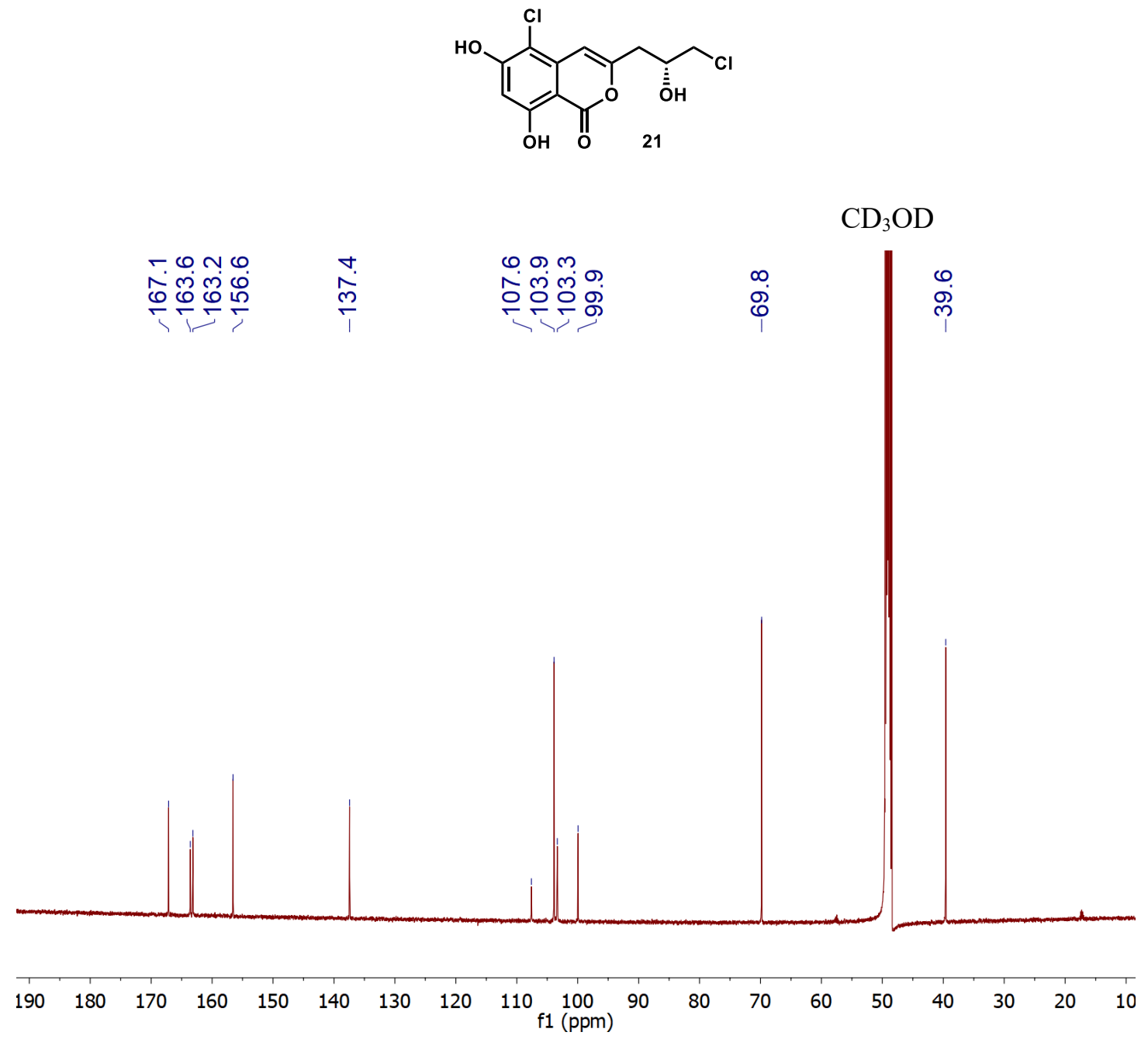

Figure S96. The ${ }^{13} \mathrm{C}$ NMR spectrum of compound 21 in $\mathrm{CD}_{3} \mathrm{OD}$. 

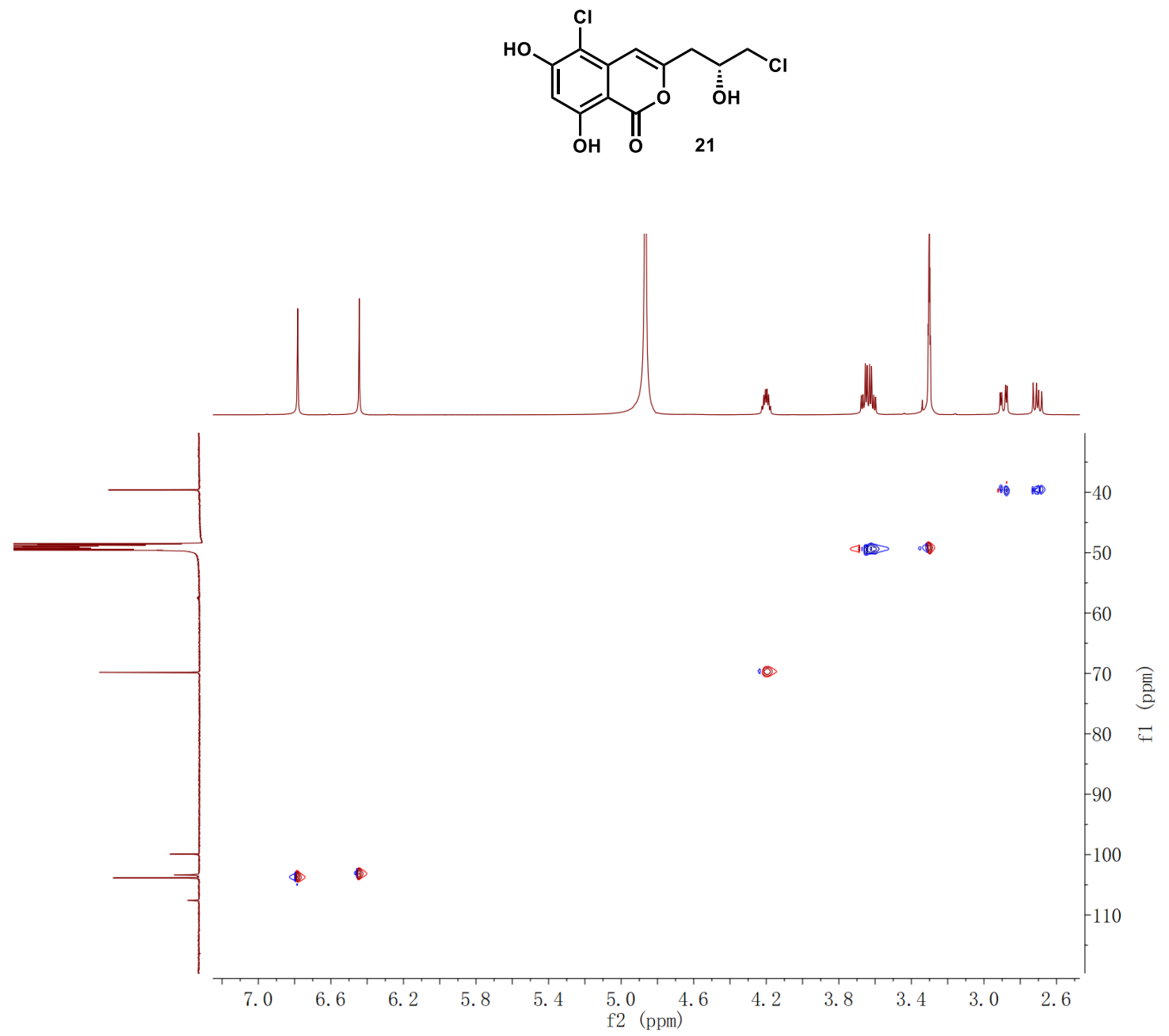

Figure S97. The HSQC spectrum of compound 21 in $\mathrm{CD}_{3} \mathrm{OD}$. 


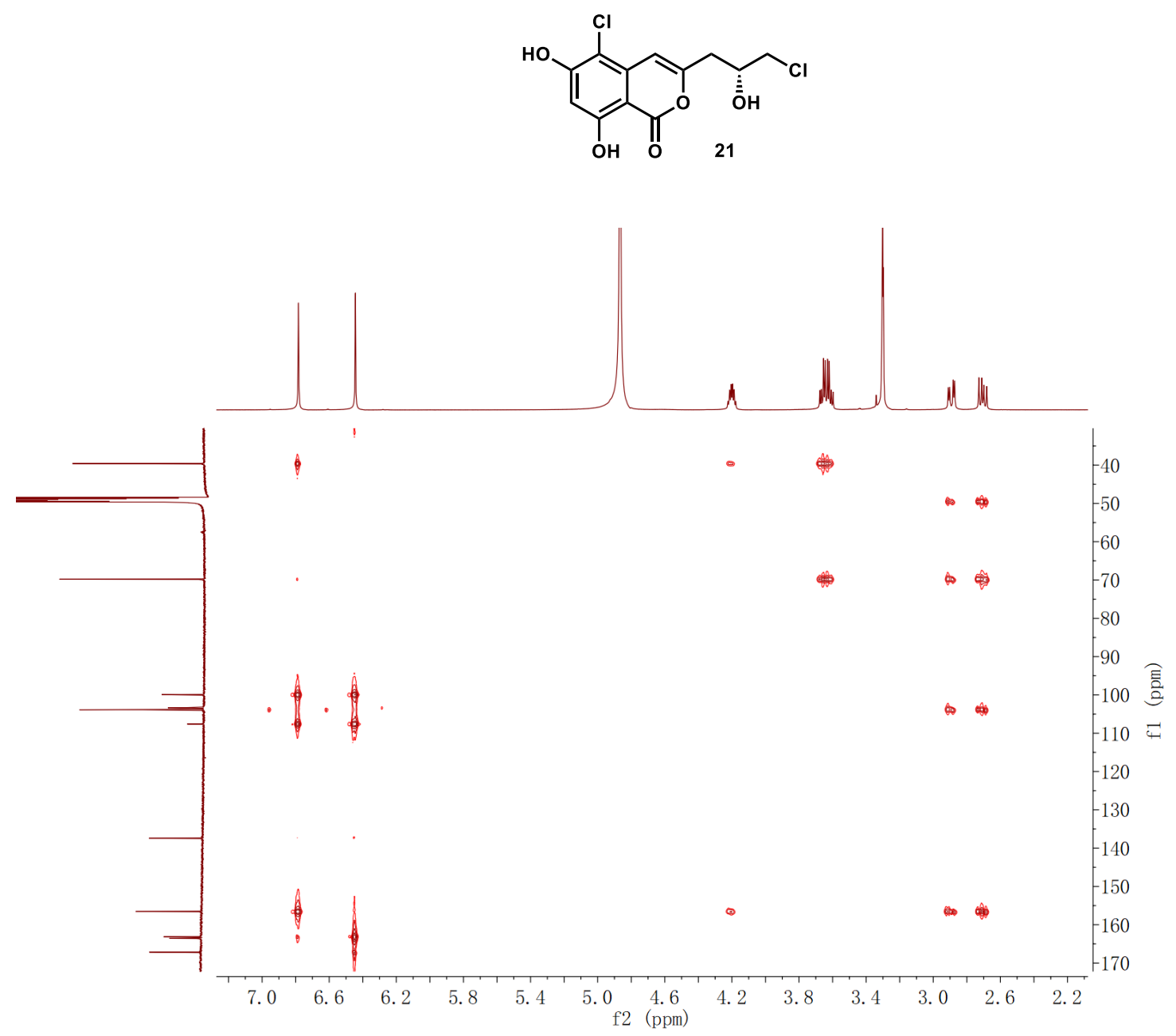

Figure S98. The HMBC spectrum of compound 21 in $\mathrm{CD}_{3} \mathrm{OD}$. 


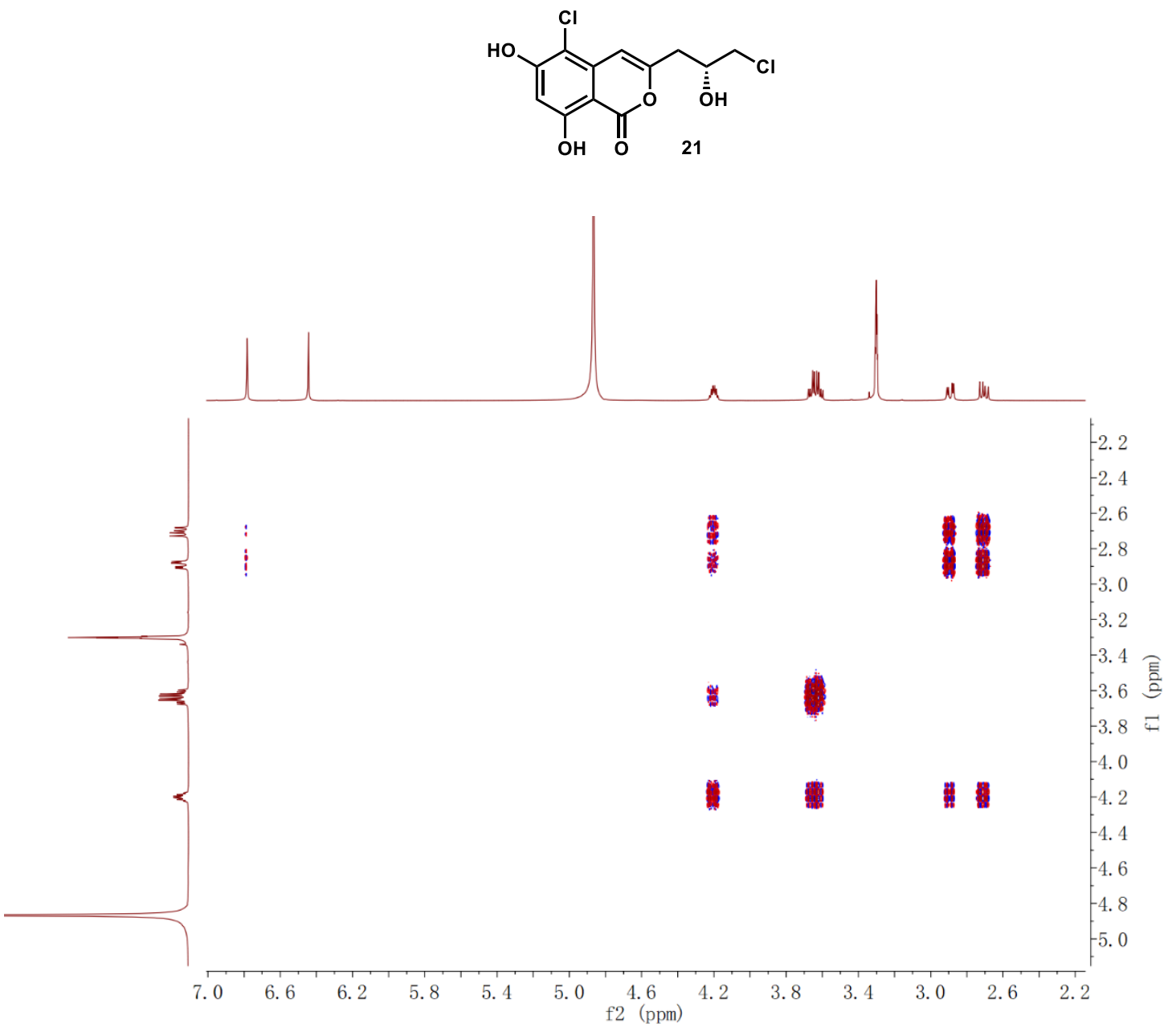

Figure S99. The ${ }^{1} \mathrm{H}-{ }^{1} \mathrm{H}$ COSY spectrum of compound 21 in $\mathrm{CD}_{3} \mathrm{OD}$. 

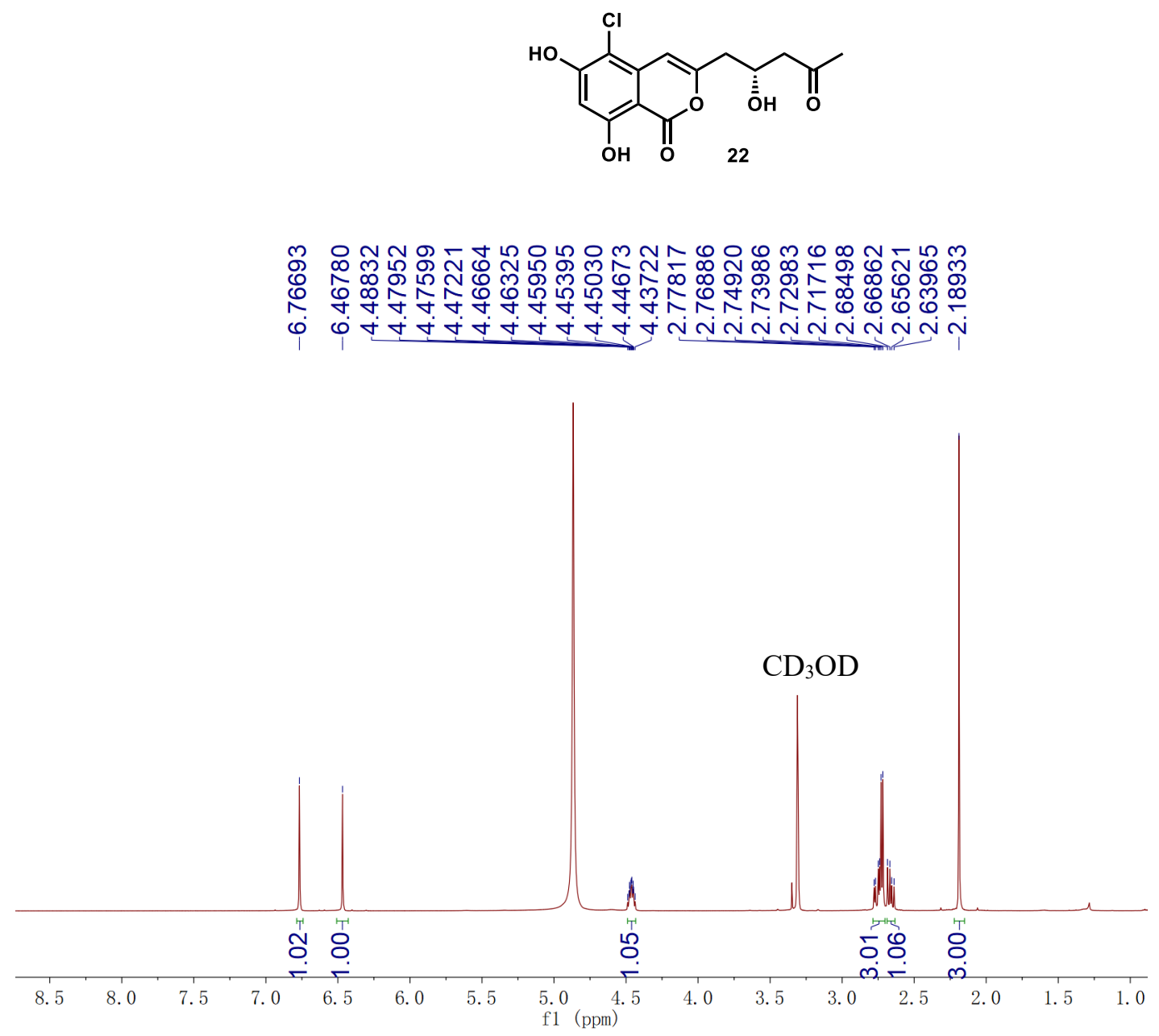

Figure S100. The ${ }^{1} \mathrm{H}$ NMR spectrum of compound 22 in $\mathrm{CD}_{3} \mathrm{OD}$. 

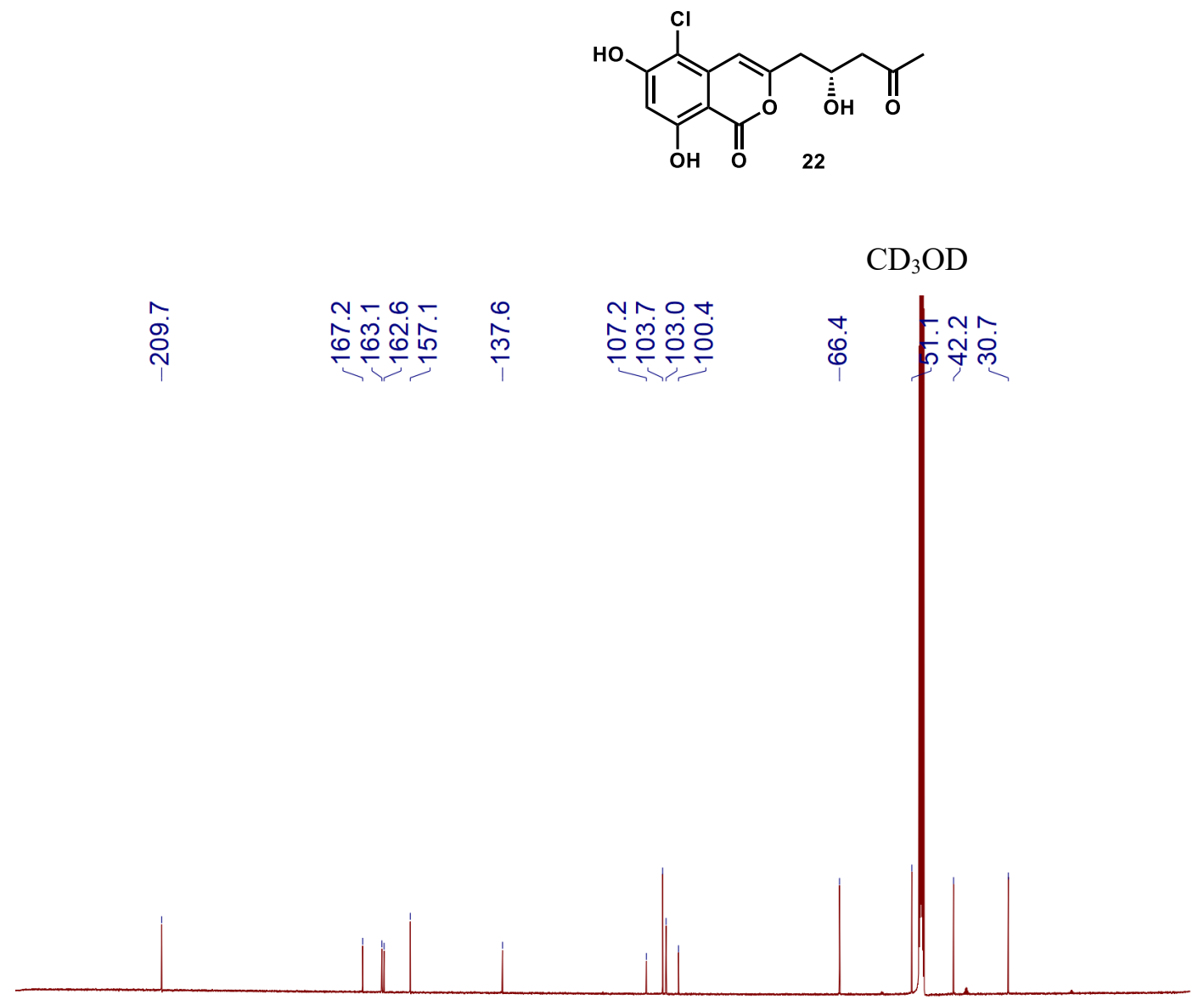

$40230220210200190180170160150140130 \begin{array}{lllllllllllllll}120 & 110 & 100 & 90 & 80 & 70 & 60 & 50 & 40 & 30 & 20 & 10 & 0\end{array}$

Figure S101. The ${ }^{13} \mathrm{C}$ NMR spectrum of compound 22 in $\mathrm{CD}_{3} \mathrm{OD}$. 


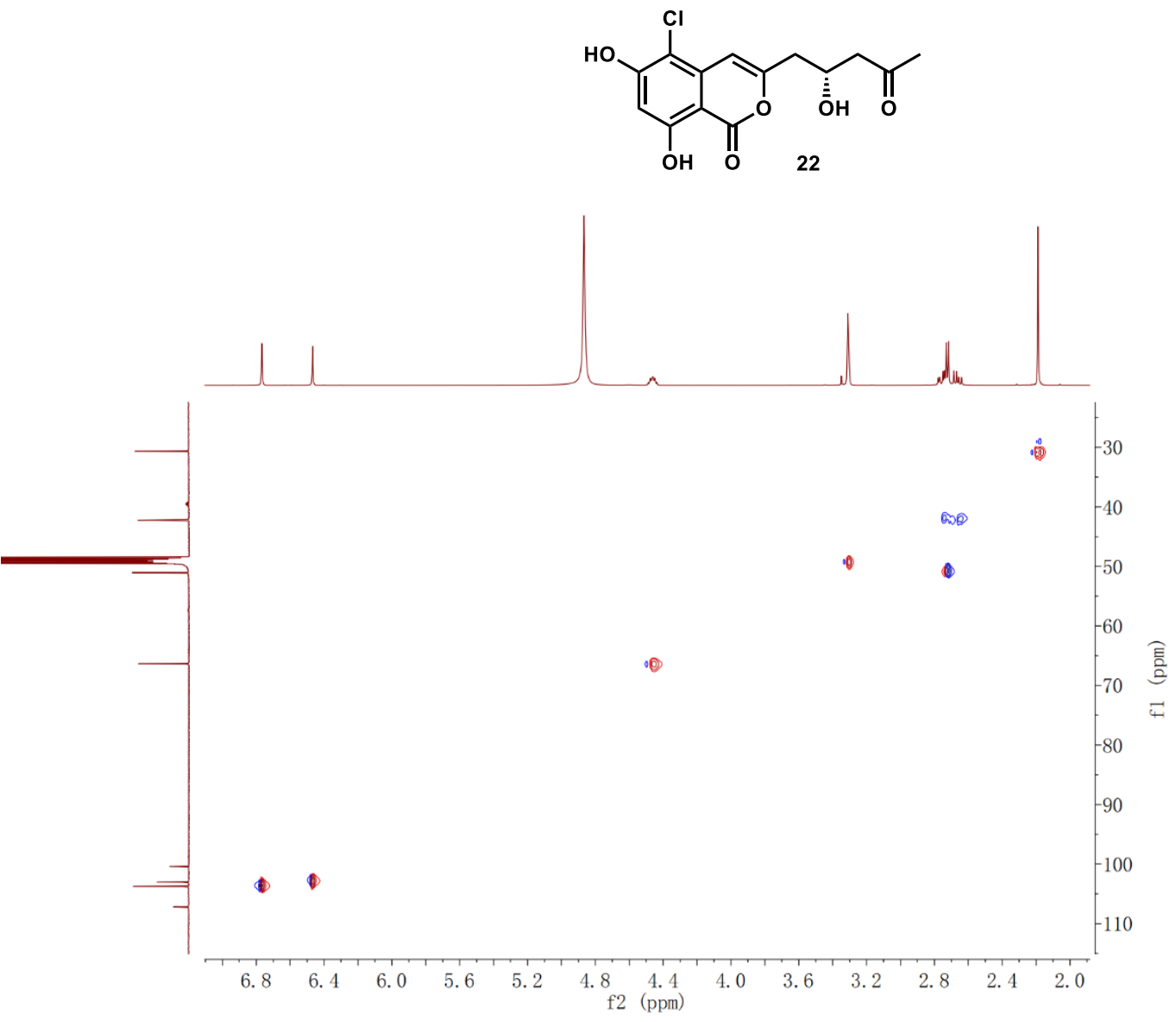

Figure S102. The HSQC spectrum of compound 22 in $\mathrm{CD}_{3} \mathrm{OD}$. 

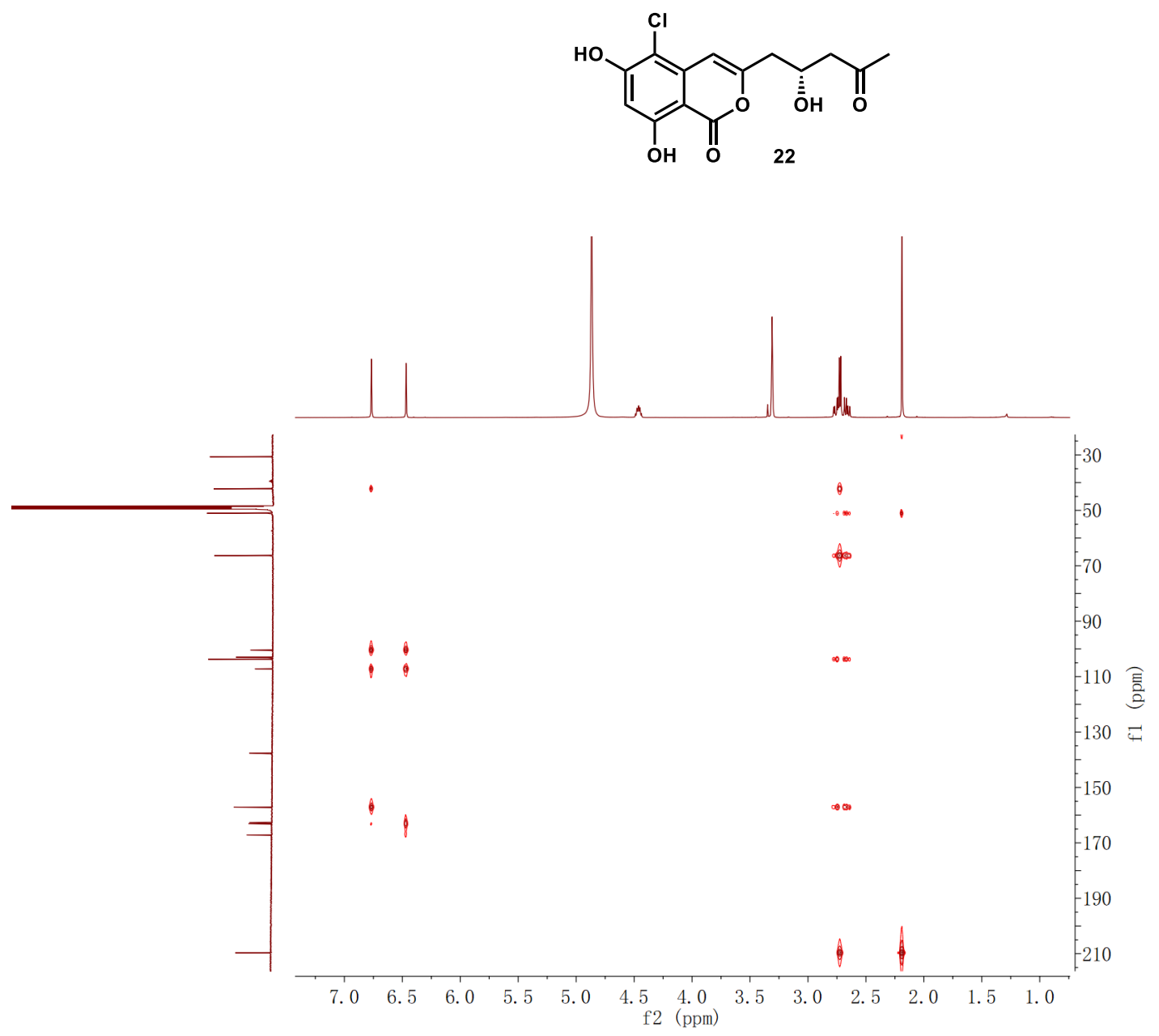

Figure S103. The HMBC spectrum of compound 22 in $\mathrm{CD}_{3} \mathrm{OD}$. 


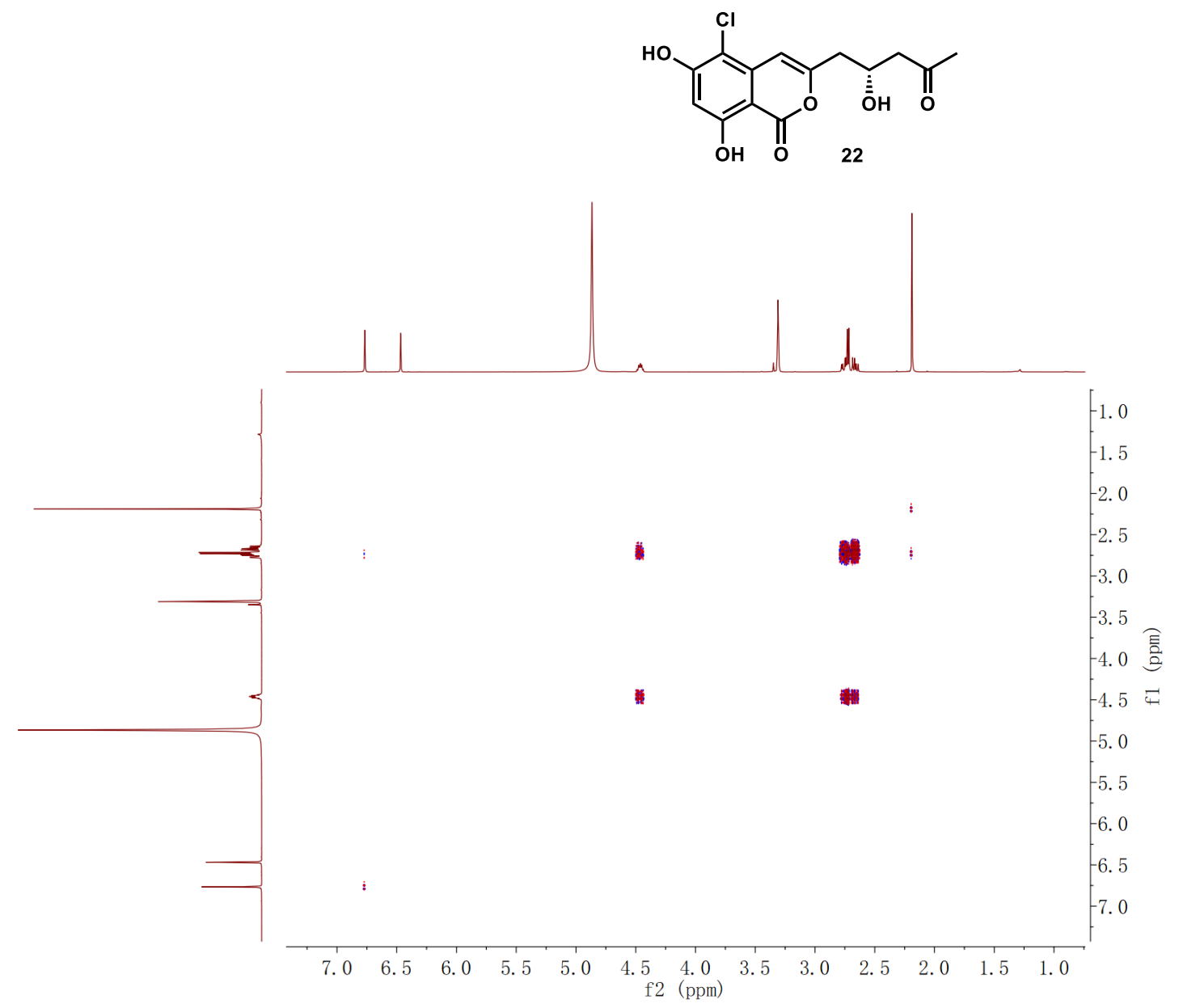

Figure S104. The ${ }^{1} \mathrm{H}-{ }^{1} \mathrm{H}$ COSY spectrum of compound 22 in $\mathrm{CD}_{3} \mathrm{OD}$. 

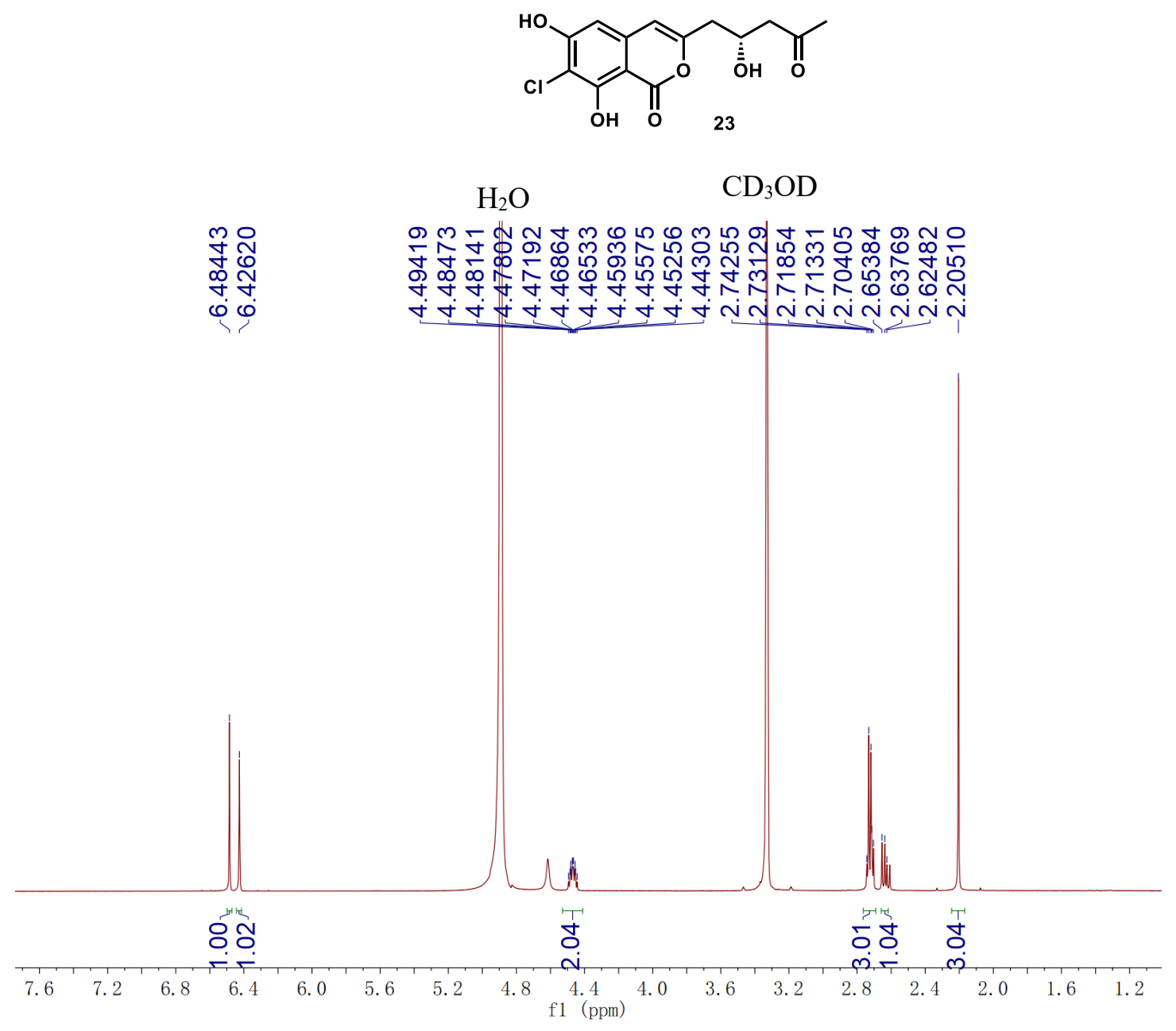

Figure S105. The ${ }^{1} \mathrm{H}$ NMR spectrum of compound 23 in $\mathrm{CD}_{3} \mathrm{OD}$. 

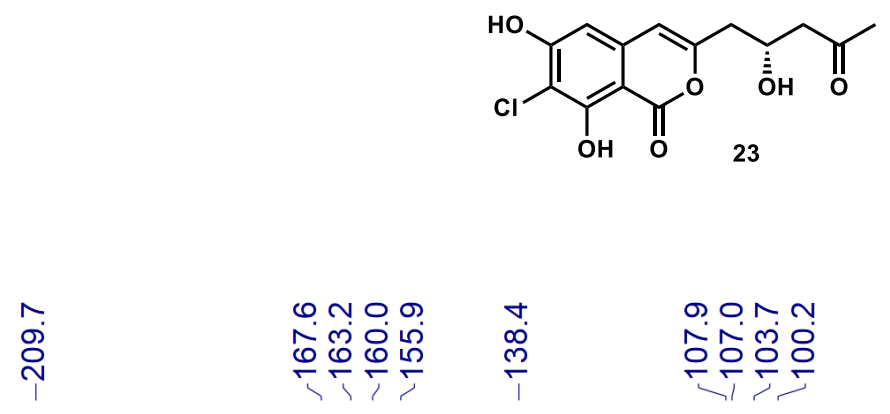

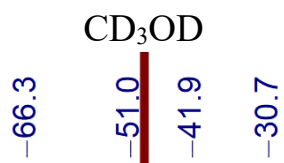

$\begin{array}{llllllllllllllllllllll}: 30 & 220 & 210 & 200 & 190 & 180 & 170 & 160 & 150 & 140 & 130 & 120 & 110 & 100 & 90 & 80 & 70 & 60 & 50 & 40 & 30 & 26\end{array}$

Figure S106. The ${ }^{13} \mathrm{C}$ NMR spectrum of compound 23 in $\mathrm{CD}_{3} \mathrm{OD}$. 

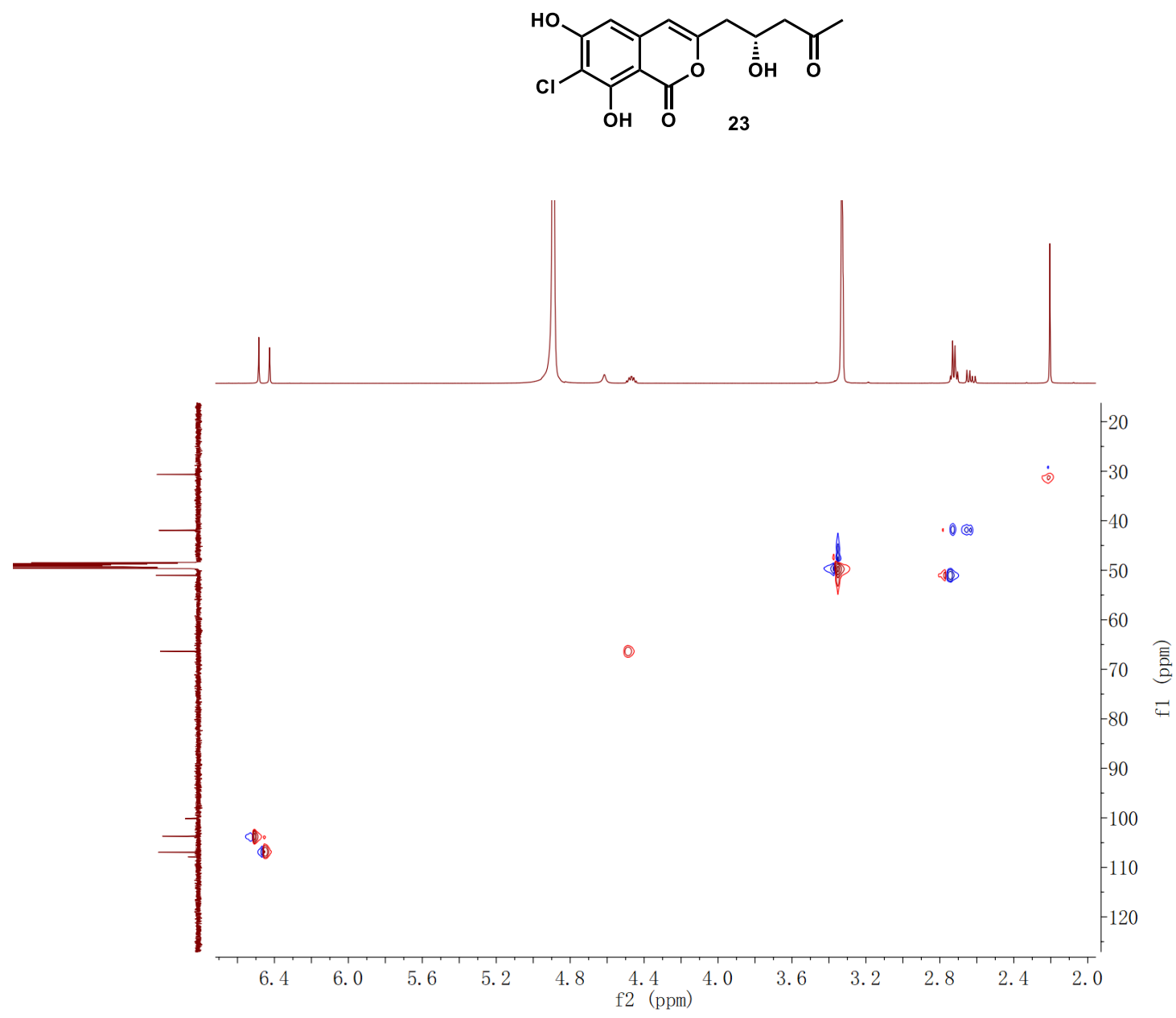

Figure S107. The HSQC spectrum of compound 23 in $\mathrm{CD}_{3} \mathrm{OD}$. 


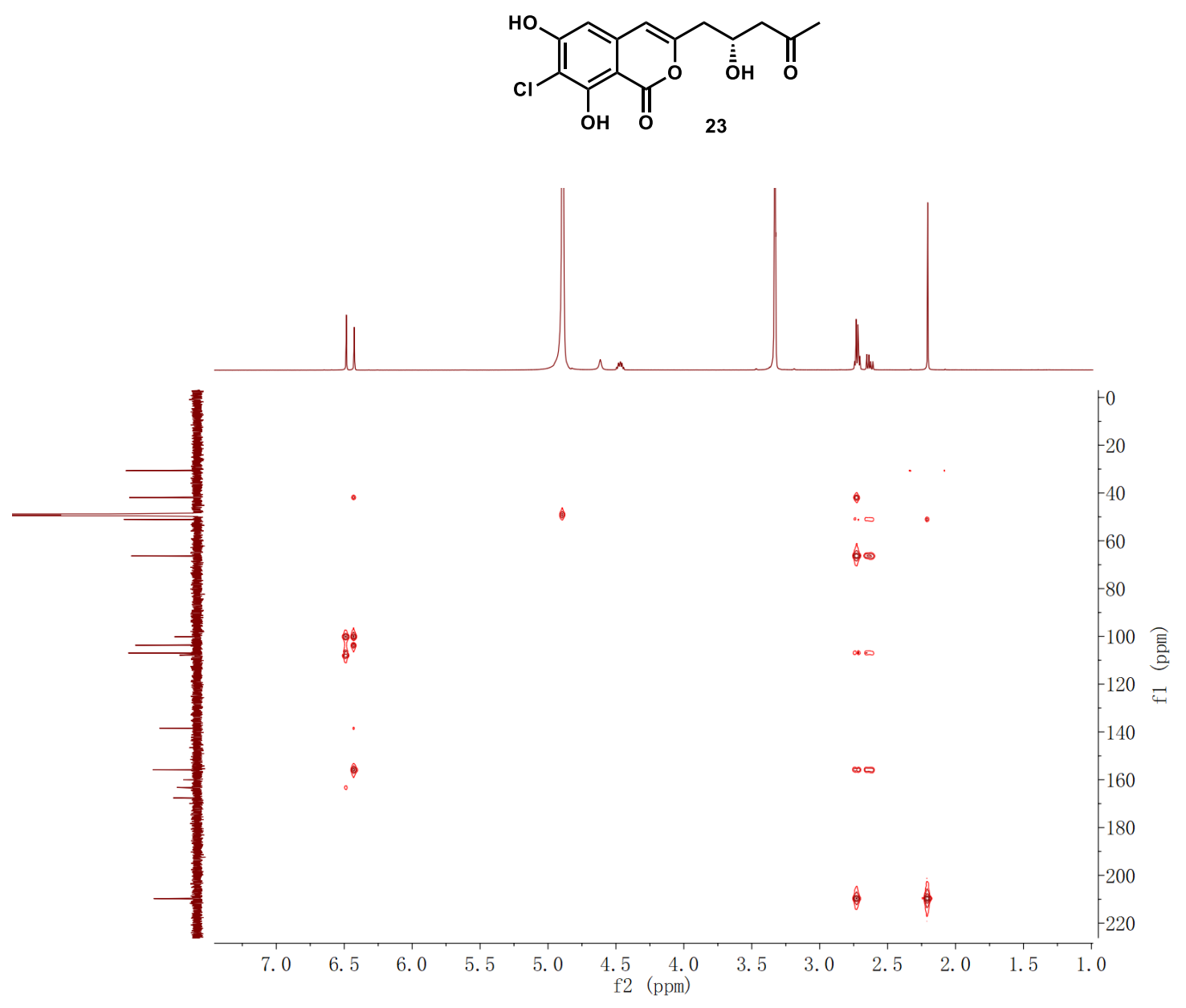

Figure S108. The HMBC spectrum of compound 23 in $\mathrm{CD}_{3} \mathrm{OD}$. 

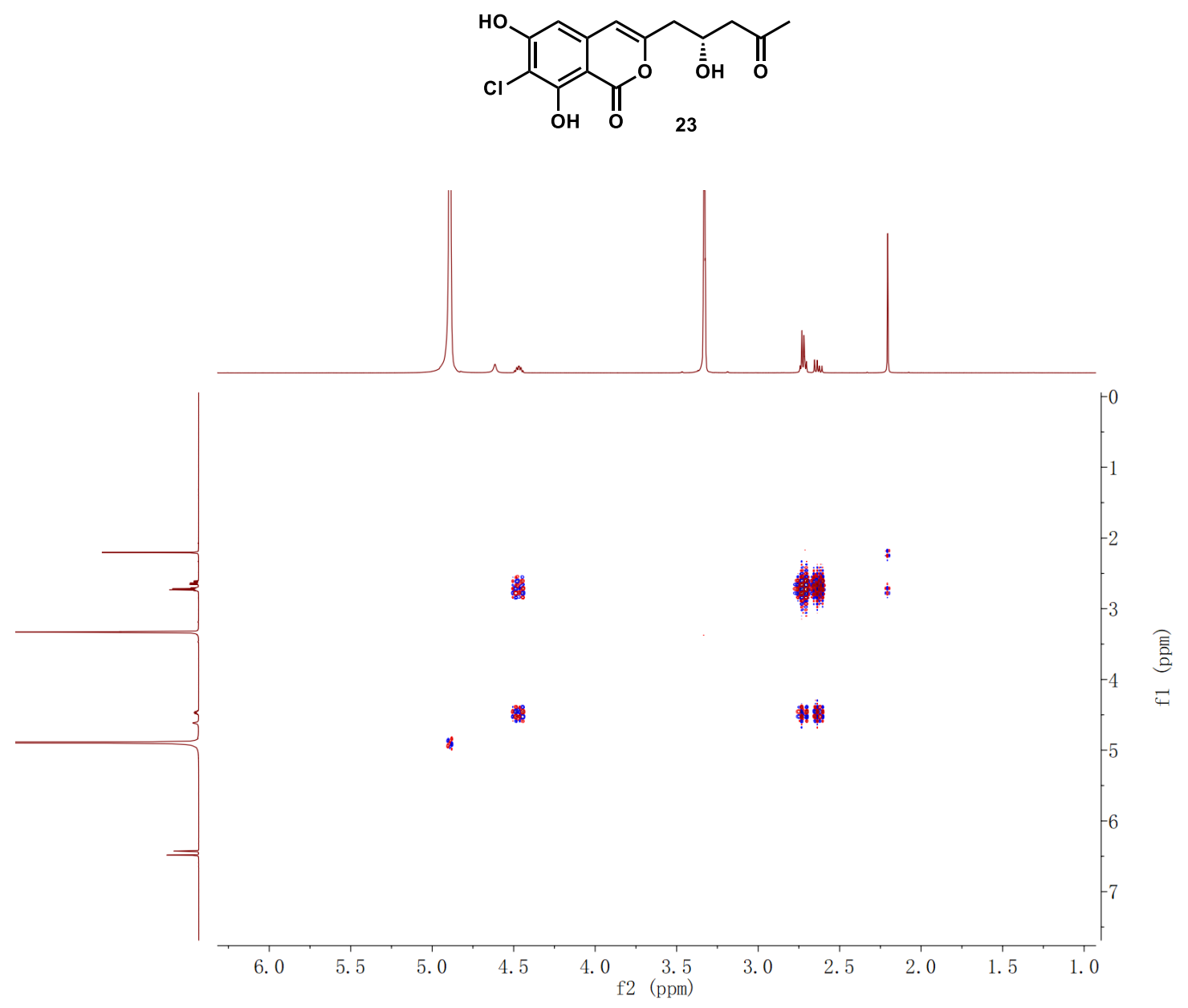

Figure S109. The ${ }^{1} \mathrm{H}-{ }^{1} \mathrm{H}$ COSY spectrum of compound 23 in $\mathrm{CD}_{3} \mathrm{OD}$. 

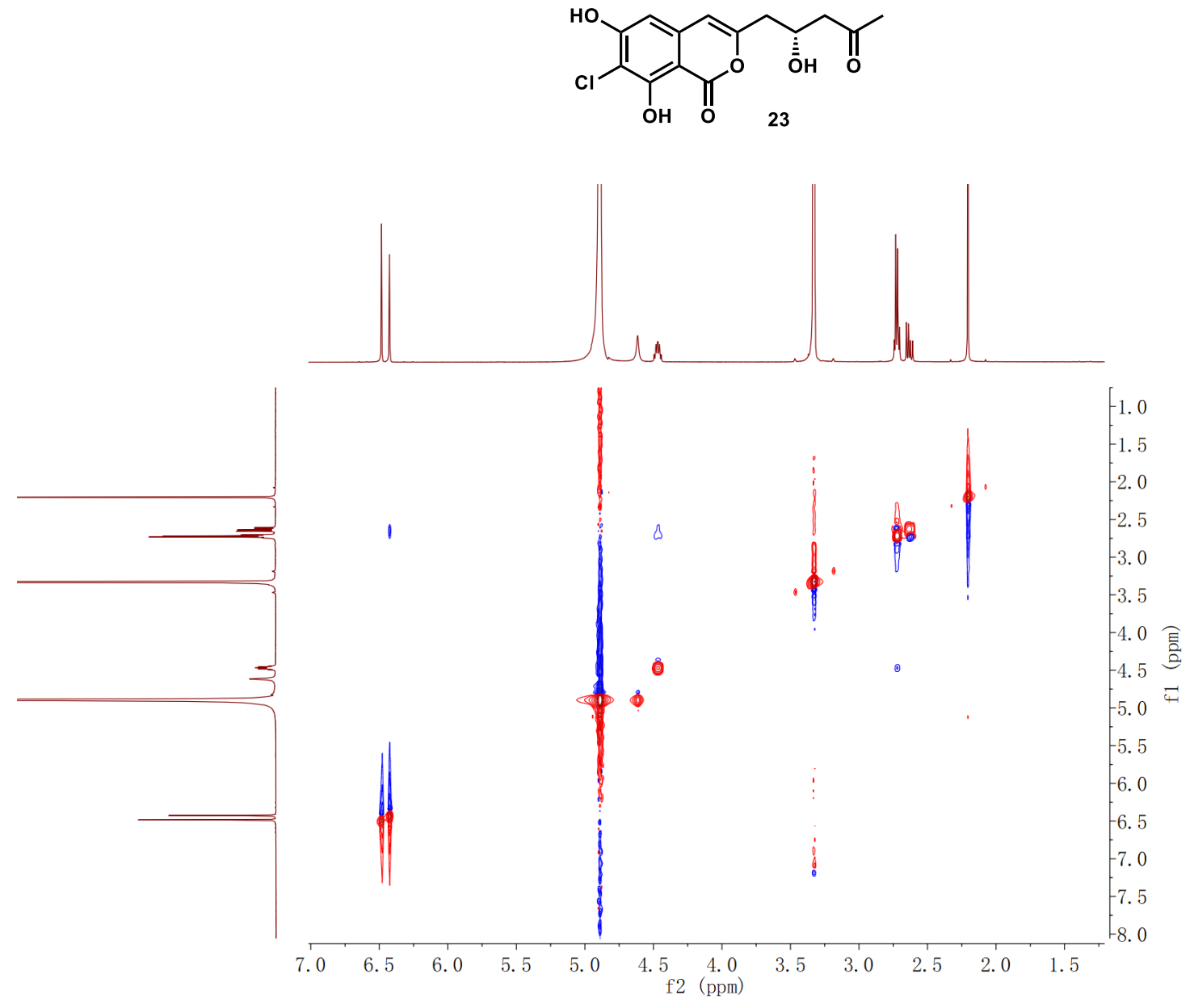

Figure S110. The NOESY spectrum of compound 23 in $\mathrm{CD}_{3} \mathrm{OD}$. 


\section{Supplementary References}

(1) Liu, N.; Hung, Y.-S.; Gao, S.-S.; Hang, L.; Zou, Y.; Chooi, Y.-H.; Tang, Y. Org. Lett. 2017, 19, 3560-3563.

(2) Li, L.; Yu, P.; Tang, M.-C.; Zou, Y.; Gao, S.-S.; Hung, Y.-S.; Zhao, M.; Watanabe, K.; Houk, K. N.; Tang, Y. J. Am. Chem. Soc. 2016, 138, 15837-15840.

(3) S. M.; Li, J. W.-H.; Choi, J. W.; Zhou, H.; Lee, K. K. M.; Moorthie, V. A.; Xie, X.; Kealey, J. T.; Silva, N. A. D.; Vederas, J. C.; Tang, Y. Science 2009, 326, 589-592.

(4) Yan, Y.; Liu, Q.; Zang, X.; Yuan, S.; Bat-Erdene, U.; Nguyen, C.; Gan, J.; Zhou, J.; Jacobsen, S. E.; Tang, Y. Nature 2018, 559, 415-418.

(5) Kim, J.-J.; Kweon D.-H.; Cho, S.-D.; Kim, H.-K.; Lee, S.-G.; Yoon, Y.-J. Synlett 2006, 2, 194-200.

(6) Chankhamjon, P.; Tsunematsu, Y.; Ishida-Ito, M.; Sasa, Y.; Meyer, F.; Boettger-Schmidt, D.; Urbansky, B.; Menzel, K. D.; Scherlach, K.; Watanabe, K.; Hertweck, C. Angew. Chem. Int. Ed. 2016, 55, 11955-11959.

(7) Larsen, T. O.; Breinholt, J. J. Nat. Prod. 1999, 62, 1182-1184.

(8) Aly, A. H.; Edrada-Ebel, R.; Wray, V.; Müller, W. E. G.; Kozytska, S.; Hentschel, U.; Proksch, P.; Ebel, R. Phytochemistry 2008, 69, 716-1725.

(9) Zhang, X.-Q.; Qu, H.-R.; Bao, S.-S.; Deng, Z.-S.; Guo, Z.-Y. Chemistry of Natural Compounds, 2020, 56, 3032.

(10) Nakazawa, T.; Ishiuchi, K.; Praseuth, A.; Noguchi, H.; Hotta, K.; Watanabe, K. ChemBioChem 2012, 13, 855861.

(11) Fang, F.; Zhao, J.-Y.; Ding, L.-J.; Huang, C.-H.; Naman, C. B.; He, S.; Wu, B.; Zhu, P.; Luo, Q.-J.; Gerwick, W. H.; Yan, X.-J.; Wang, Q.-W.; Zhang, Z.-J.; Cui, W. Marine drugs 2017, 15, 260.

(12) Mallampudi, N. A.; Choudhury, U. M.; Mohapatra, D. K. J. Org. Chem. 2020, 85, 4122-4129.

(13) Latham, J.; Brandenburger, E.; Shepherd, S. A.; Menon, B. R. K.; Micklefield, J. Chem. Rev. 2018, 118, 232-269.

(14) Chankhamjon, P.; Boettger-Schmidt, D.; Scherlach, K.; Urbansky, B.; Lackner, G.; Kalb, D.; Dahse, H.-M.; Hoffmeister, D.; Hertweck, C. Angew. Chem. Int. Ed. 2014, 53, 13409-13413.

(15) Chooi, Y.-H.; Cacho, R.; Tang, Y. Chemistry \& Biology, 2010, 17, 483-494.

(16) Yeh, E.; Garneau, S.; Walsh, C. T. PNAS, 2005, 102, 3960-3965.

(17) Yeh, E.; Blasiak, L. C.; Koglin, A.; Drennan, C. L.; Walsh, C. T. Biochemistry 2007, 46, 1284-1292.

(18) Kong, L.; Wang, Q.; Deng, Z.; You, D. Appl. Environ. Microbiol. 2020, 86 (18). 